\title{
Toxicological consequences of the Maillard reaction
}

Citation for published version (APA):

van der Lugt, T. (2020). Toxicological consequences of the Maillard reaction: dietary advanced glycation endproducts and the gastrointestinal tract. [Doctoral Thesis, Maastricht University]. Maastricht University. https://doi.org/10.26481/dis.20200918tl

Document status and date:

Published: 01/01/2020

DOI:

10.26481/dis.20200918t|

Document Version:

Publisher's PDF, also known as Version of record

\section{Please check the document version of this publication:}

- A submitted manuscript is the version of the article upon submission and before peer-review. There can be important differences between the submitted version and the official published version of record.

People interested in the research are advised to contact the author for the final version of the publication, or visit the DOI to the publisher's website.

- The final author version and the galley proof are versions of the publication after peer review.

- The final published version features the final layout of the paper including the volume, issue and page numbers.

Link to publication

\footnotetext{
General rights rights.

- You may freely distribute the URL identifying the publication in the public portal. please follow below link for the End User Agreement:

www.umlib.nl/taverne-license

Take down policy

If you believe that this document breaches copyright please contact us at:

repository@maastrichtuniversity.nl

providing details and we will investigate your claim.
}

Copyright and moral rights for the publications made accessible in the public portal are retained by the authors and/or other copyright owners and it is a condition of accessing publications that users recognise and abide by the legal requirements associated with these

- Users may download and print one copy of any publication from the public portal for the purpose of private study or research.

- You may not further distribute the material or use it for any profit-making activity or commercial gain

If the publication is distributed under the terms of Article $25 \mathrm{fa}$ of the Dutch Copyright Act, indicated by the "Taverne" license above, 


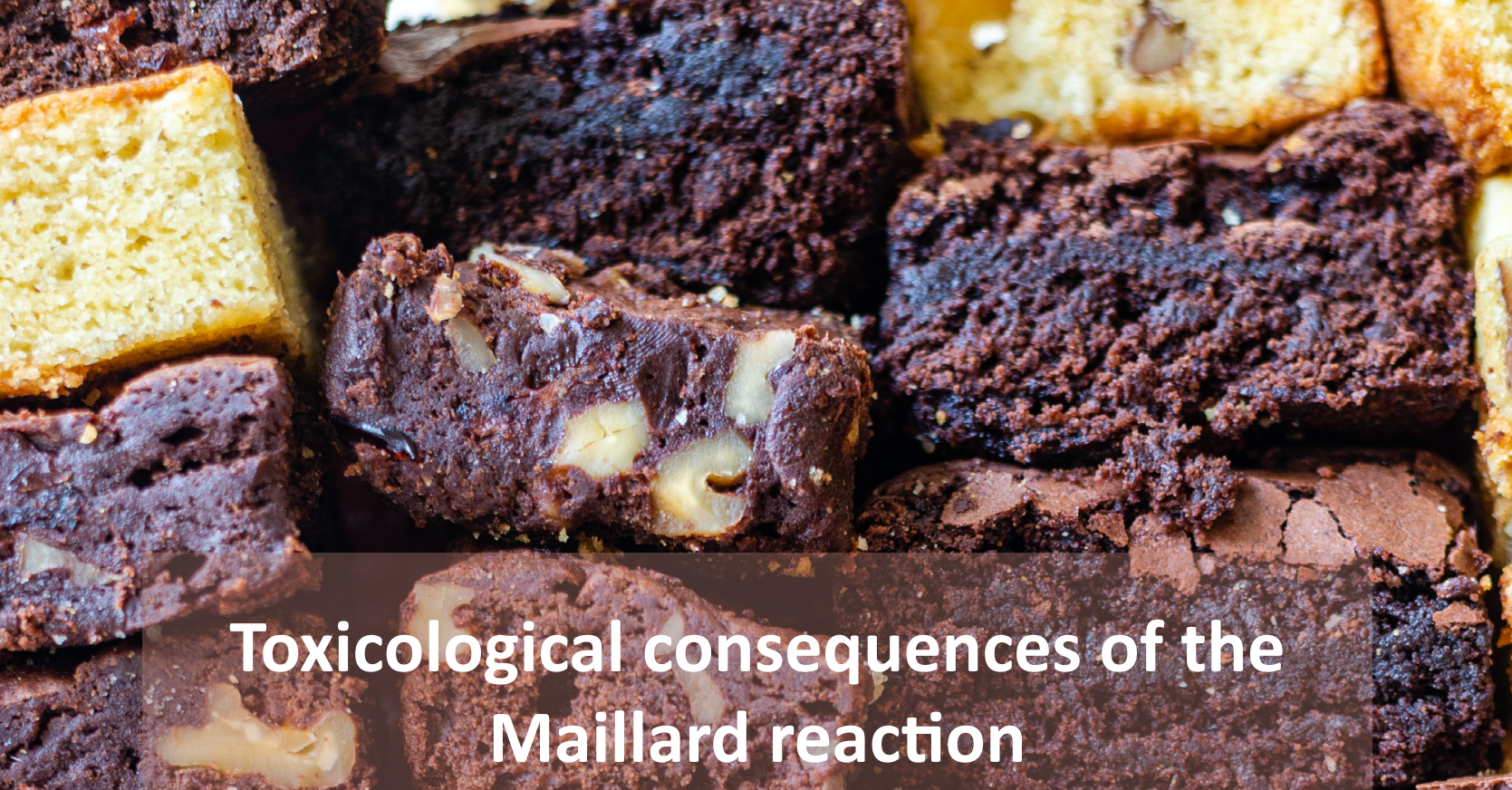

\section{Toxicological consequences of the Maillardreaction}

W. Dietary advanced glycation endproducts and the gastrointestinal tract

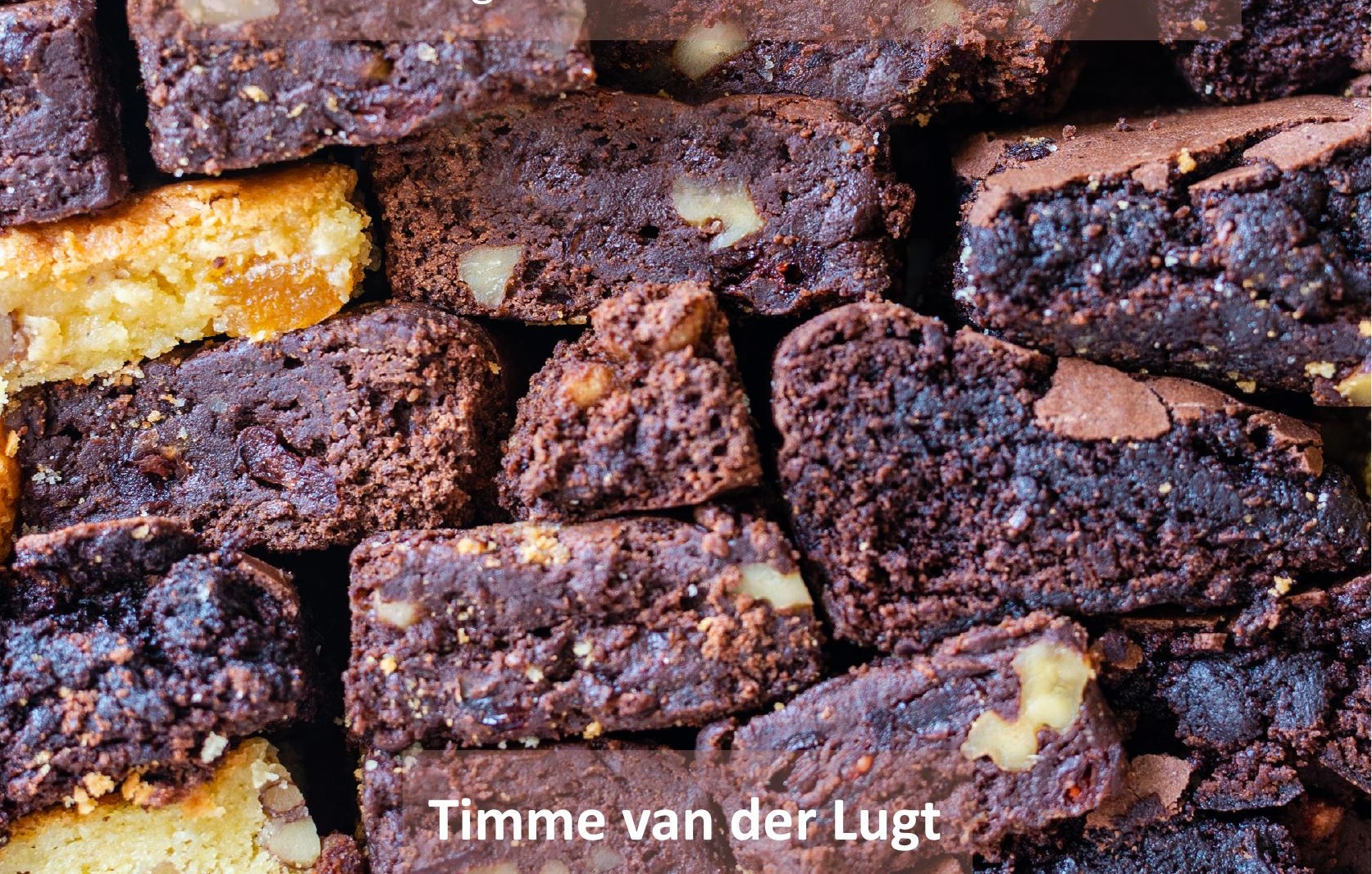

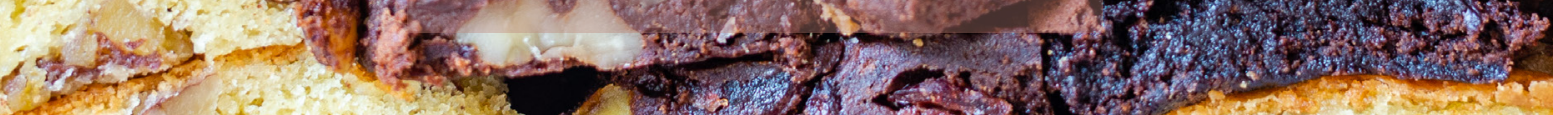
sis
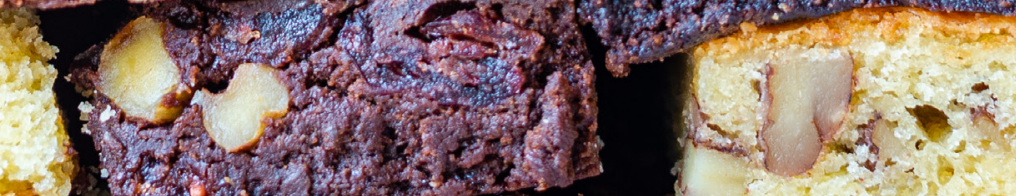


\section{$\mathscr{0}$ NUTRIM}

(C) Timme van der Lugt, 2020.

Cover photo: Jan van Prooije

Cover design: Jan van Prooije, Timme van der Lugt

Layout: Timme van der Lugt

Chemical structures: Martien Würdemann

Printed by: Gildeprint, Enschede

ISBN: $\quad$ 978-94-6402-429-6

The research described in this thesis was conducted at NUTRIM School of Nutrition and Translational Research in Metabolism at Maastricht University, Wageningen Food Safety Research (WFSR) at Wageningen University, and the Dutch Food and Consumer Product Safety Authority (NVWA). 


\title{
Toxicological consequences of the Maillard reaction
}

\author{
Dietary advanced glycation endproducts \\ and the gastrointestinal tract
}

\section{Proefschrift}

Ter verkrijging van de graad van doctor aan de Universiteit Maastricht, op het gezag van de Rector Magnificus, Prof. dr. Rianne M. Letschert, volgens het besluit van het College van Decanen, in het openbaar te verdedigen op vrijdag

18 september om 10:00 uur

door

\section{Timme van der Lugt}

Geboren te Amsterdam op 17 februari 1990, Nederland 


\section{Promotores}

Prof. dr. A. Bast

Prof. dr. A. Opperhuizen

\section{Co-promotor}

Dr. M. F. Vrolijk

\section{Beoordelingscommissie}

Prof. dr. C. G. Schalkwijk (voorzitter)

Dr. H. Deluyker (ex-Scientific Advisor of the European Food Safety Authority)

Prof. dr. D. M. A. E. Jonkers

Prof. dr. D. T. H. M. Sijm

Prof. dr. R. F. Witkamp (Wageningen University) 
Voor papa 



\section{Table of contents}

Chapter $1 \quad$ General Introduction 9

Chapter $2 \quad$ Dietary advanced glycation endproducts induce 35 an inflammatory response in human macrophages in vitro

Chapter 3 Gastrointestinal digestion of dietary advanced glycation endproducts using an in vitro model of the gastrointestinal tract (TIM-1)

Chapter $4 \quad$ The pro-inflammatory potential of dietary advanced glycation endproducts after gastrointestinal digestion

Chapter 5 Dietary advanced glycation endproducts decrease glucocorticoid sensitivity in vitro

Chapter 6 Summary and Discussion

Chapter $7 \quad$ Samenvatting

Valorisatie

Dankwoord

Curriculum Vitae

List of publications 


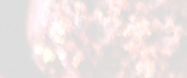
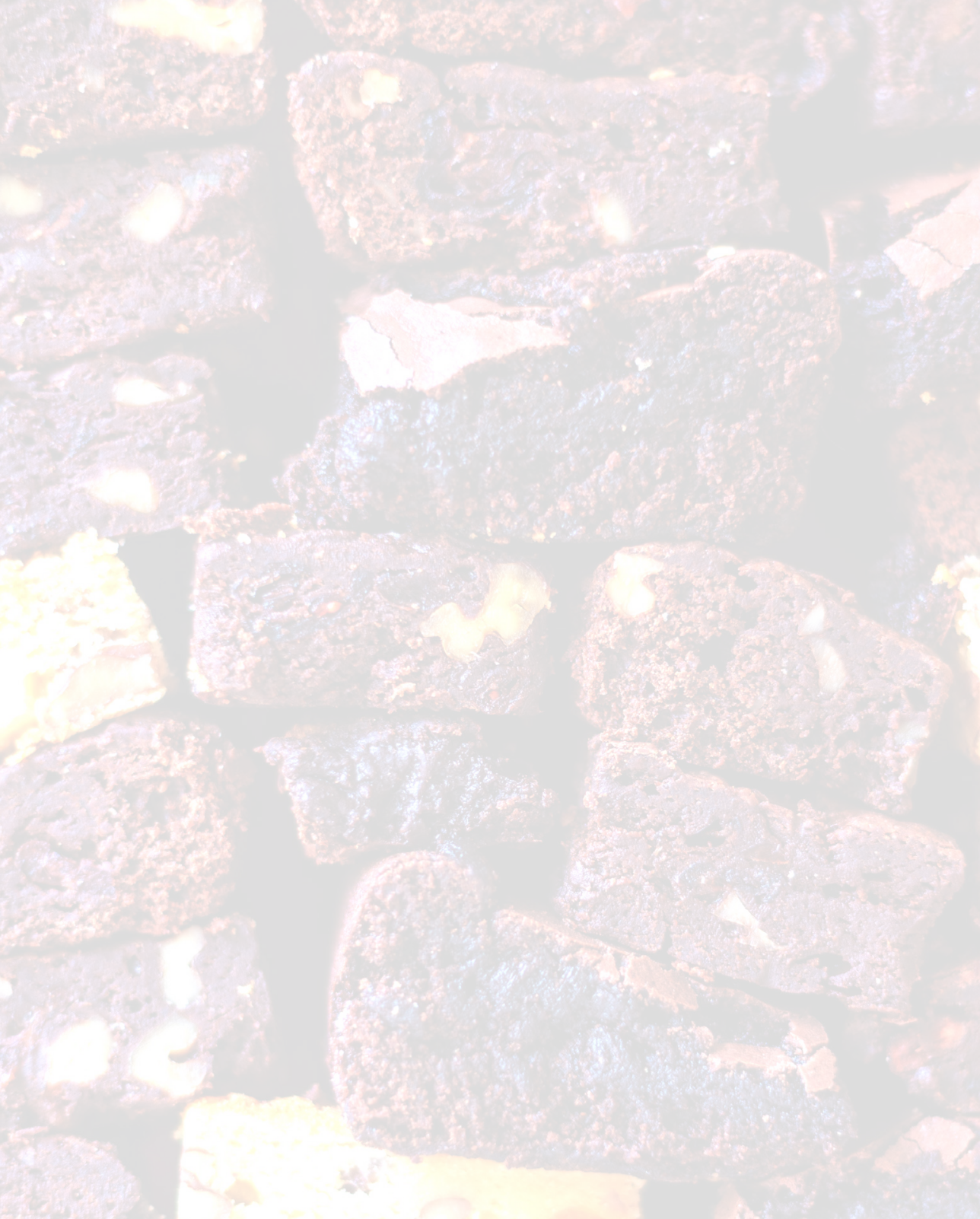


\section{Chapter 1}

\section{General introduction}




\section{Food toxicology}

Nutrition is a primary necessity, safe nutrition is therefore of pivotal importance to human health and wellbeing. Humans have always been practicing food toxicology. In pre-historic times, humans were hunter-gatherers; berries picked could be poisonous and hunted meat could become spoiled. Consuming, and thus testing, gathered and hunted food could lead to illness and even death. In later times, food toxicology would be practiced by food tasters that were employed by monarchs. Evidence of the existence of food tasters goes back to ancient Rome and are even still employed in the modern world. Famous state heads employing food tasters are for instance the Russian president Vladimir Putin and most, if not all, US presidents [1]. Not all food contaminants make you ill instantly. Most food-induced illnesses occur slowly and are more due to an unhealthy diet than to bacterial contamination and poison. The current Western diet generally considered an unhealthy diet. It includes high levels of sugars and fats and is usually highly processed. This all contributes to the development of the metabolic syndrome that is associated with a higher risk of cardiovascular diseases and many other diseases [2]. 


\section{The Maillard reaction}

\subsection{The reaction}

During food processing, new compounds are introduced to food and process contaminants are being formed. Additionally, food products are often enriched with sugars and fats; proteins are often added to obtain foods that may help build muscle. All of these can potentially impact human health. The processes that food products undergo often include industrial heat treatments, such as sterilization, but also cooking and baking at home. During thermal treatment of foods that contain proteins and carbohydrates, the Maillard reaction (MR) can occur. This is a chemical reaction between reducing sugars and amino acids, which is responsible for the browning of food. The MR is an intricate reaction with multiple parallel and sequential steps, as presented in figure 1. In the first initial stage, a carbonyl (from reducing sugars, oxidized lipids, vitamin $\mathrm{C}$ or quinones) condensates with an amine moiety in amino acids and proteins, and leads to the formation of a Schiff base adduct. Following this stage, the Schiff base adduct is rearranged into an Amadori product in the intermediate stage. Amadori products are unstable and prone to polymerization due to being highly unsaturated. In the final stage, both sequential and parallel reactions lead to different molecules. These reactions include oxidation, dehydration, enolization, cyclization, and fragmentation [3]. During the MR many different classes of compounds are formed. These compounds are also known as MR products (MRPs), which include Amadori products, early glycation products, advanced glycation endproducts (AGEs) and melanoidins.
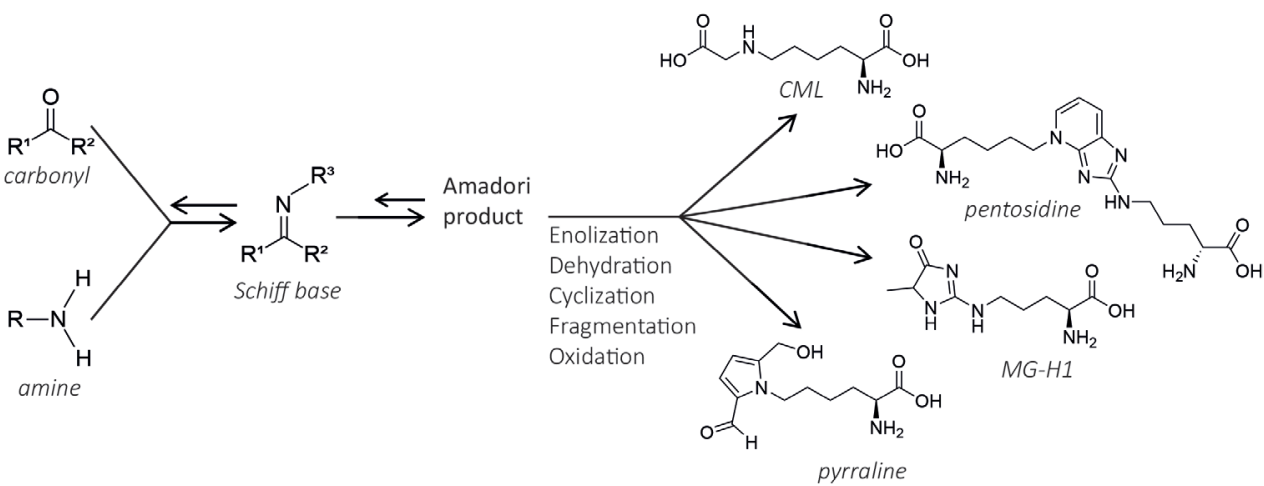

Figure 1. Simplified overview on AGE formation via the Maillard reaction. As an example, the molecular structure of four different AGEs are shown.

Especially the subgroup AGEs which are formed during one of the last stages of the MR have received attention lately. The term AGEs implies a whole group of different molecules. Some well-known AGEs are: $\mathrm{N} \varepsilon$-carboxymethyl-lysine (CML), NE-carboxyethyl-lysine (CEL), methylglyoxal-derived hydroimidazolone-1 (MG-H1), pentosidine, and pyrraline. Within the large group of different AGEs it is possible to make a distinction between low molecular weight (LMW) and high molecular weight (HMW) AGEs. LMW AGEs consist of up to four 
linked rings weighing up to $1 \mathrm{kDa}$ [4]. HMW AGEs are assumed to be water-soluble and coloured, weighing up to $150 \mathrm{kDa}$ [4]. Additionally, AGEs exist in both a protein-bound form and a free form and their visible colour is brown. The formation of AGEs has been nicely explained by Poulsen et al. (2013) [3]. AGEs do not only form during the MR, other reactions may lead to the formation of AGEs as well. Intermediate compounds that can form AGEs are 1,2-dicarbonyl derivatives such as glyoxal (GO), methylglyoxal (MG), and 3-deoxyglucosone (3-DG), which can interact with monoacids to form AGEs. MG and GO can be formed during caramelization, while through the radical Namiki pathway (lipid oxidation) 1,2-dicarbonyls can also be formed [5]. Fructose metabolites such as fructose-3-phosphate are formed in the polyol pathway which are then converted into $\alpha$-oxaldehydes. $\alpha$-Oxaldehydes can form AGEs by interacting with monoacids [6, 7]. The formation of MRPs can be monitored by the formation of brown colours, as Hofmann et al. (1998) stated that the browning intensity seemed to be parallel with cross-linking of casein [8].

\subsection{Factors influencing the MR}

The MR and other pathways through which AGEs form, can be influenced by different factors. AGEs formed in food products are named dietary AGEs (dAGEs). An important factor influencing the rate of $\mathrm{dAGE}$ formation is the macronutrient composition of the food products. The presence of sugar leads to both initiation of the MR and caramelization. Proteins are made up from amino acids, so their presence increases the rate of the MR as well. Lipids can be oxidized, leading to the formation of 1,2-dicarbonyls via the Namiki pathway [3]. One factor relating to this is the reactivity of the amino groups themselves. Primary amines are more reactive than secondary amines, whereas tertiary amines are inactive [9]. The primary amino group present in the side-chains of lysine is the most reactive precursor amine in proteins. Next to this, the guanidino group of arginine, the indole group of tryptophan, and the imidazole group of histidine are reactive sites, but to a lesser extent. Practically any $\mathrm{N}$-terminal amino group and free amino group is reactive [10]. The sugars that react in the MR are reducing sugars. These are sugars with a free aldehyde or ketone group such as glucose, galactose and fructose [11]. Besides these groups, other chemical characteristics of sugars, carbohydrates, and amino acids also determine their reactivity in the MR. In a very early study by Ashoor et al. (1984), the browning of common amino acids in combination with different sugars was assessed and the different amino acids were categorized by high, middle, and low reactivity [12]. The high reactivity group included Lys, Gly, Trp, and Tyr. The middle group included Pro, Leu, Ile, Ala, Hypro, Phe, Met, Val, Gln, Ser, and Asn. The amino acids with the lowest reactivity in their study were His, Thr, Asp, Arg, Glu, and Cys [12]. Although the high reactivity of lysine is shown in several studies, the level of reactivity of the other amino acids is under dispute [10, 13-15]. Kwak et al. (2004) analysed the browning of all possible combinations between 12 different amino acids with five different sugars (glucose, fructose, maltose, xylose, and arabinose), which is an indication of MRP formation. Lysine showed the highest formation of MRPs with all five sugars. The order of reactivity of the other 
amino acids depended on the type of sugar: pentoses were for instance more reactive than hexoses [14]. Most commonly analysed and used dAGEs are therefore formed from lysine and arginine. In the case of glucose, arginine was indeed the second most reactive amino acid. However, the reaction between glucose and arginine did not lead to a brown colour, despite the fact that glucose was depleted quickly. By using a peptide library (SPOT library) to assess the reactivity of different amino acid residues to glucose, fructose and AGE-BSA, Münch et al. (1999) also found that the binding reactivity of amino acids depended on the type of sugar or dAGE provided [10]. In the case of glucose and fructose, amino acids with nucleophilic side chains (lysine, cysteine, and histidine) were prone to bind these sugars, whereas AGE-BSA preferentially bound to arginine and tryptophan and to a lesser extent to lysine. This difference in binding reactivity between dAGEs and reducing sugars could be an explanation why both lysine and arginine based protein-bound dAGEs are largely present in food products.

To identify the products formed during the MR, Hemmler et al. (2018) recently used a nontargeted analysis with Fourier transform ion cyclotron resonance mass spectrometry (FT-ICRMS) to derive the molecular formulae of compounds formed after $10 \mathrm{~h}$ of heating at $100^{\circ} \mathrm{C}$ for different sugar-amino acid combinations [15]. The reaction rate order that was found for amino acids was: lysine > cysteine $>$ isoleucine $\approx$ glycine. Interestingly, when assessing the browning of the different combinations, the order of reactivity was lysine $>$ isoleucine $>$ glycine > cysteine. Showing that cysteine produces many non-brown MRPs. The order of reaction rates for the sugars was: ribose $>$ arabinose $>$ fructose $\approx$ xylose $>$ galactose $>$ glucose. Joubran et al. (2017) confirmed the higher galactose reactivity compared to glucose in combination with $\alpha$-lactalbumine. Which can be explained by the equatorial orientation of the hydroxyl groups in glucose and glucose' stable closed-ring structure. The open chain-form of sugars react more quickly than the closed ring [16]. Important in the sugar reactivity is also the proportion of free carbonyls and the length of the carbon backbone. As seen earlier in the study of Kwak et al. (2004), pentoses have a faster reaction rate than hexoses. Next to this, the monosaccharides have a higher reactivity than disaccharides, with disaccharide being more reactive than oligosaccharides $[16,17]$.

Next to the composition of the raw materials, baking conditions influence the formation of dAGEs to a large extent [18]. An increase in temperature leads to an increased formation of dAGEs. The compounds formed during the different pathways are very reactive, but for the reaction to continue the molecules must react with each other. Therefore, diluting these molecules in aqueous solutions slows down the formation of dAGEs. Also, $\mathrm{H}+$ ions are capable of binding to the intermediate reactants, making them less reactive and slowing down the formation of dAGEs. These effects have nicely been reviewed by Lund et al. (2017) [17]. The $M R$ is an ongoing reaction and therefore does not only occur during the processing of food products, but also during the storage of food. Storage conditions apparently influence the rate of the MR, like temperature and moisture/humidity level [19]. Since 1,2-dicarbonyls can 
be formed via the Namiki pathway from the oxidation of fat, the presence of oxygen in the packaging can accelerate the MR, while addition of antioxidants may hamper the rate of the MR due to trapping of oxidation products. A practical example of the importance of starting product in the MR is the difference between goat's milk infant formula and cow's milk infant formula. Infant formulas from goat's milk have a lower CML content than from cow's milk, consequently also heat processing leads to lower levels of CML in goat's milk compared to cow's milk [20].

As mentioned earlier, Amadori products, early glycation products, AGEs and melanoidins all fall under the umbrella term 'MRPs'. Hence, all these compounds are being formed during thermal processing of food rich in carbohydrates and proteins. Therefore, a problem that arises with studies into the effect of dAGEs when using food matrices, is the simultaneous formation of this broad and varying subset of molecules and the unclear border between these different subsets. dAGEs have defined molecular structures such as CML, CEL, MG$H 1$, pentosidine, and pyrraline. A second subset of molecules formed in the end-stage of the MR are melanoidins, which have a higher molecular weight than dAGEs. The dAGEs are considered to be the reactive intermediates, whose condensation forms melanoidins [21, 22]. The use of the terms 'AGEs' and 'melanoidins' in existing literature is not always fully clear. dAGEs contribute to the brown colour of thermally treated food and some of them have fluorescent properties. Remarkably, melanoidins are also defined as brown, high molecular weight heterogeneous polymers, and are usually analysed in food products by measuring the intensity of the browning and fluorescence [21]. It is therefore almost impossible to out rule the presence of dAGEs in those studies measuring melanoidin levels only. The presence and ratio of the different subsets in food products is dependent on the food matrix and the above discussed processing conditions. The difficult distinction within the end-stage of the MR makes it difficult to attribute the effects of heated foods to specific compounds. In this chapter we will try to distinguish between the different subsets and focus on the effects of dAGEs as a subset but will include the effects of MRPs in general since all of these compounds are present in food, but attempt to make a distinction in the terminology where possible. When discussing literature, it will be specifically stated which subset of compounds were investigated.

\subsection{Assessment of dietary AGEs in food products}

In order to define the characteristics of dAGE-rich food products, it is important to know in what kind of food products they are present. This brings us to an important problem in current literature. Since dAGEs are a heterogeneous group of compounds, it makes it difficult to analyse them very specifically. Different methods are being used throughout literature to determine the amount of dAGEs present in different food products. Several AGEs have fluorescent properties and the fluorescence spectrum of vesperlysine ( $\lambda$ ex $=370 \mathrm{~nm} / \lambda$ em $=440 \mathrm{~nm}$ ) is considered as an AGE-wide specific fluorescence in multiple studies [23-25]. A major drawback of this method is that not all AGEs have fluorescent properties. For instance 
$\mathrm{CML}$, which is regarded in current literature as the most important dAGE in food, does not have any fluorescent properties [26]. A different technique used in multiple studies to detect dAGE presence is competitive ELISA [27]. Both ELISA and fluorescence assays are easy to perform but have a low accuracy in measuring dAGEs. As stated before, dAGEs are a group of different compounds and for ELISA you need specific antibodies for specific molecules. Goldberg et al. (2004) made a database of food products measured with an AGE ELISA using anti-CML antibodies [27]. It is however questionable what specific molecules are recognized by the anti-AGE antibodies. The values obtained in these studies are expressed as AGE-kU, for which the definition of AGE-kU remains unclear. It is therefore only possible to compare trends between food products within the same study. More specific analytical techniques are based on chromatography and mass spectrometry. UPLC-MS/MS can more specifically measure specific molecules and quantify them. Other, less often used, techniques are nuclear magnetic resonance (NMR) spectroscopy [28] and size exclusion chromatography combined with fluorescence [29]. All methods used in the analysis of MRPs have been recently reviewed by Troise [30]. Interestingly, the use of different techniques leads to different results. Figure 2 depicts the average CML content of different food groups per average portion size, comparing the results of the ELISA database of Goldberg et al. (2004) and the mass spectrometry database of Hull et al. (2012) [27,31]. As can be seen, the trend between the two methods is completely different. This was also seen by Niquet-Léridon et al. (2015), who showed lower $\mathrm{CML}$ levels and other $\mathrm{CML}$ patterns when comparing food groups, especially in the fat and carbohydrate food groups, when measured by ELISA compared to the LC-MS/MS measurement [32].

(A)

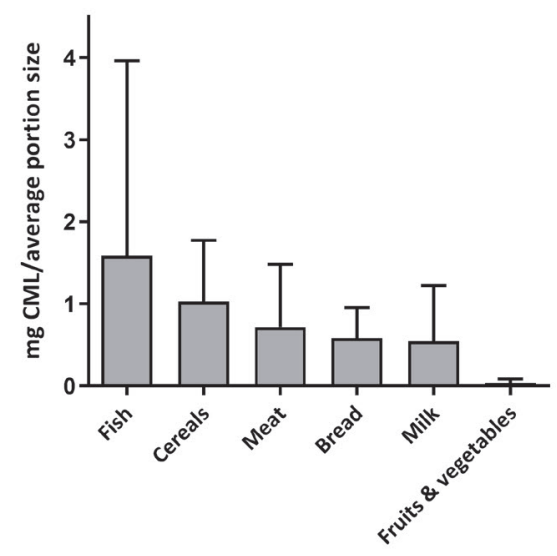

(B)

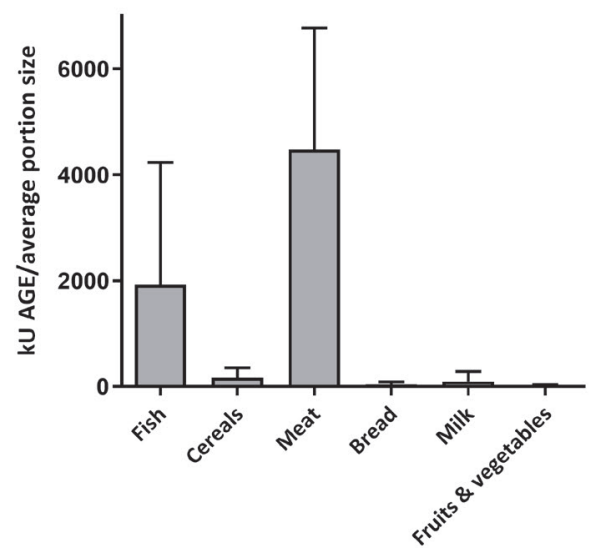

Figure 2. Difference in AGE content of food products using different quantification methods. (A) Data from Hull et al. (2012) [31]. (B) Data from analyses using CML antibodies from Goldberg et al. (2004) [27]. 
Several databases exist on the amount of dAGEs in food products measured by mass spectrometry. Scheijen et al. (2016) assessed CML, CEL, and MG-H1 levels in the Dutch food products [33], Delatour et al. (2009) assessed CML in dairy products [34], and Hull et al. (2012) assessed a vast range of food products from the Western diet on CML levels [31]. The precursors 3-DG, 3-deoxygalactosone, MG, and 5-hydroxymethylfurfural (HMF) have been assessed by Degen et al. (2012) [35]. Table 1 shows a selection of food products relatively high in dAGE content, based on the database published by Scheijen et al. (2016).

Additionally, although the databases published give good indications on the levels of specific dAGEs in food products, they do not make a distinction between free and protein-bound dAGEs. Protein-bound dAGEs are in general much more abundant in food products than free dAGEs, as was recently reviewed by Zhao et al. (2019) [36]. The ratios in which dAGEs are present in either free or protein-bound form seems to depend on the type of food product, as was shown in the study by Hegele et al. (2008) who compared levels of free and proteinbound dAGEs in raw and processed milk products [37]. In samples of pasteurized milk, especially the early glycation product $\mathrm{N} \varepsilon$-fructoselysine was found to be up to 800 -fold higher present in protein-bound form compared to free form, protein bound CML was found in levels 30-fold higher than its free form. Levels of dAGEs in pasteurized and ultra-high temperature (UHT) treated milk did not differ significantly from levels found in raw milk. Increasing the processing however led to much higher levels of dAGEs as in the case of condensed milk. Processed food proteins generally have $2 \%$ of their lysine modified. However, in samples like heated milk and bread crust this can be up to 10-20\% [38].

Table 1. Average CML, CEL, and MG-H1 content of several food products as published by Scheijen et al. (2016) [33].

\begin{tabular}{llll}
\hline Food product & CML (mg)/100g & CEL $(\mathbf{m g}) / \mathbf{1 0 0 g}$ & MG-H1 (mg)/100g \\
\hline Blood sausages & 4.8 & 7.7 & 63.0 \\
\hline Peanut butter & 3.1 & 6.7 & 44.5 \\
\hline Cereals & 2.0 & 1.6 & 41.6 \\
\hline Ginger biscuit & 2.5 & 2.0 & 28.3 \\
\hline Salted peanuts & 1.7 & 3.4 & 25.7 \\
\hline Rusk & 2.0 & 1.4 & 23.1 \\
\hline Red cooked beef & 2.0 & 5.6 & 13.5 \\
\hline Chocolate sprinkles & 5.1 & 2.0 & 9.3 \\
\hline "Hagelslag" & & 2.8 & 11.0 \\
\hline Canned salmon & 1.2 & 1.2 & 10.9 \\
\hline Fried tofu & 0.9 & & \\
\hline
\end{tabular}




\section{Endogenous AGEs}

As stated before, the specific MRP subset AGEs has gained a lot of attention in recent years due to their potential negative effect on human health. Existing literature on AGEs mostly focuses on endogenously formed AGEs, which form when endogenous sugars react with endogenous proteins at $37^{\circ} \mathrm{C}$. The health effects of endogenous AGEs, including inflammation, tissue damage, stiffening of collagen, and enhancement of oxidative stress, have been studied thoroughly [5]. AGEs can bind to different receptors, of which the receptor for advanced glycation endproducts (RAGE) is the most well known receptor. Under normal physiological conditions, RAGE is expressed at low levels in all organs, except in lung tissue, which has a high baseline expression of RAGE [39]. Under pathophysiological conditions, such as diabetes, inflammatory diseases, and neurodegenerative disorders, RAGE is strongly upregulated in different tissues, such as vasculature, central nervous system and gastrointestinal tract [39-41]. RAGE is a $35 \mathrm{kDa}$ transmembrane receptor and member of the immunoglobulin superfamily, a large protein superfamily that shares structural features with antibodies [42]. Different variants of RAGE exist, of which full length RAGE is the only variant that is both capable of ligand binding and signal transduction. Full length RAGE consists of the extracellular site of a V-type domain for ligand binding and two C-type domains of which the exact function is unknown. RAGE is integrated in the cell membrane with a transmembrane spanning helix, and intracellularly consists of a C-terminal cytosolic domain for signal transduction [40,43,44]. RAGE is proposed to be a pattern recognition receptor and has many different ligands next to AGEs, among which are proteins with high inflammatory potentials such as high mobility group box 1 (HMGB1), lipopolysaccharides (LPS), the signalling molecule lysophosphatic acid, damage-associated molecular patterns, and advanced oxidation protein products [39, 40,43, 45, 46]. RAGE signalling and outcome has been discussed in several reviews $[40,47]$. RAGE signalling leads to several different (patho)physiological outcomes including, but not limited to, inflammation, oxidative stress, apoptosis, proliferation, and autophagy [40]. Upon extracellular ligand binding and activation, a small intracellular protein, mDia1, binds to the cytosolic C-domain of RAGE, activating nuclear factor kappa-light-chainenhancer of activated B cells (NF-KB) through phosphoinositide 3-kinase (PI3K), eventually leading to the release of an array of pro-inflammatory cytokines such as interleukin (IL)-1B, IL-6, tumour necrosis factor (TNF)- $\alpha$, and chemokine ligand 2 (CCL2). This was confirmed by blockage of RAGE, which resulted in delaying the inflammatory response $[40,48]$. In macrophages, ligand binding to RAGE induces a pro-inflammatory phenotype, resembling the pattern of $\mathrm{M} 1$ polarized macrophages, and stimulated the migration of monocytes and leukocytes through the endothelium [49]. RAGE signalling furthermore results in a positivefeedback loop through activation of NF-KB, which in turn upregulates RAGE expression [6, 39]. In addition to RAGE, AGEs bind to a variety of different other receptors, which are mainly implicated in regulating endocytosis and degradation of AGEs. These receptors include macrophage scavenger receptors, $80 \mathrm{~K}-\mathrm{H}$ phosphoprotein (AGE-R2), galectin-3 (AGE-R3), lectin-like oxidized low density lipoprotein receptor-1 (Lox-1), fasciclin EGF-like, laminin- 
type EGF-like, link domain-containing scavenger receptor-1/2 (FEEL1/2), and CD36 [40]. Furthermore, AGEs bind to AGER1, also known as oligosaccharyl transferase-48 (OST48). This receptor is involved in cellular uptake and degradation of AGEs and has been found to inhibit RAGE activated NF-KB activation and to mitigate the generation of reactive oxygen species (ROS) by RAGE, as well as the downstream effects [50-52]. In case of inflammatory diseases, the AGER1 to RAGE ratio seems to be decreased [53].

Apart from an inflammatory response, AGEs induce oxidative stress. After ligand binding, RAGE activates nicotinamide adenine dinucleotide phosphate (NADPH)-oxidases, eventually leading to the formation of ROS [51]. Activation of RAGE also eventually leads to an increased intracellular nitric oxide synthase (iNOS) expression [54, 55]. Next to RAGE-induced ROS, AGEs themselves are formed under oxidative conditions making them very pro-oxidant molecules [56-58]. Due to their pro-oxidative nature, AGEs react with proteins, resulting in cross-linking or other irreversible modifications, leading to loss of protein function [3]. It has been suggested that AGEs increase stiffness in muscle tissue, probably via cross-linking to collagen and elastin, leading to alterations in muscle function [59]. Other consequences are glycation of the blood-brain barrier, modification of low-density lipoprotein (LDL), impaired wound healing and non-enzymatic glycation of DNA [60,61]. 


\section{Dietary AGEs}

As stated before, AGEs specifically formed during food processing are considered as dAGEs. Differences in the health effects between endogenous AGEs and dAGEs may be expected due to the different manner of formation between both: endogenous AGEs are formed at $37^{\circ} \mathrm{C}$ with endogenous carbohydrates and endogenous proteins over a longer period of time, while dAGEs are formed with exogenous proteins and carbohydrates in a shorter period of time and under more extreme conditions, e.g. higher temperatures. Consequently, different subsets of molecules are expected to be formed. This was also shown in a recent review by Liang et al. (2019), who summarized the occurrence of different AGEs in food and in the body [62]. The systemic effects caused by endogenous AGEs can therefore not be directly extrapolated to the potential effect of dAGEs.

\subsection{Absorption}

In order to be able to assess the systemic and local effects of dAGEs, it is important to know whether dAGEs are absorbed. When dAGEs are not absorbed, they will remain in the gastrointestinal (GI) tract, travel further and potentially affect the colon. A few reviews have already included an assessment of the absorption of specific dAGEs: Delgado et al. (2015) concluded that CML is taken up to some extent. More specifically, free CML was most likely absorbed by simple diffusion, and CML bound to dipeptides by peptide transporters (PEPT1) [63]. Faist et al. (2001) and Somoza et al. (2005) both concluded that MRPs are most likely taken up, but that more research on the uptake and mechanisms is needed to confirm this. In general, HMW dAGEs may be absorbed to a much lesser extent than LMW dAGEs [64, 65]. Besides these reviews, several in vitro studies on the absorption mechanism of specific AGEs have been published and their results are summarized in table 2. Most free dAGEs are capable of entering the GI cells by diffusion, but seem to be retained inside the cell, whereas peptide-bound dAGEs are transported inside the cell by peptide transporters. The absorption of dAGEs has also been investigated in several in vivo animal studies. It should be noted that the physiological relevance of such animal studies regarding the uptake in humans is debatable, due to large differences in GI physiology between the animal species and humans. Taking the available animal studies into account, differences between free compounds and protein bound compounds exist in their ability to be absorbed. When assessing the different animal studies, free compounds seem to be taken up more easily [3], although this seems not to be the case for free CML [66]. HMW dAGEs are less absorbed due to insufficient degradation by Gl enzymes. Intragastric administration of $18 \mathrm{~F}-\mathrm{CML}$ in mice showed a $48 \%$ accumulation of CML in the intestines, $29 \%$ in bladder, and $18 \%$ in stomach, 120 min after administration [67]. Several human studies saw correlations between dAGE intake and serum AGE levels [68-70]. An intervention study with a low-AGE diet showed that changes in serum $C M L$ and the AGE precursor MG significantly correlated with changes in dAGE levels in the diet [71]. This is also seen in infants fed with formulas (IF), which contain more CML than human breast milk. Infants solely fed with IF had higher levels of CML in their blood and urine 
than breast-fed infants [72]. Additionally, urinary excretion of pyrraline, fructoselysine (both $90 \%$ ) and pentosidine (40\%) decreased in adults while consuming a diet low in dAGEs. No difference was seen for peptide-bound Amadori products [70]. A different study by Davis et al. (2015) exposed overweight and obese adults to a crossover exposure of one day to highfat, high-AGE and another day to low-fat, high-AGE diet. These acute exposures showed no important influence of dietary CML on CML serum levels, but rather an effect of high-fat diet compared to low-fat diet on CML serum levels [73]. A recent study by Scheijen et al. (2018) assessed total (no distinction between free and protein bound) dietary CML, CEL and MG$\mathrm{H} 1$ of 450 people with an elevated risk for T2DM and cardiovascular disease. A correlation between the intake of all three dAGEs and their free form levels in plasma was found. This correlation was however not found for the protein-bound plasma levels of the different dAGEs [74]. Based on this, it can be concluded that free dAGEs are indeed taken up in the systemic circulation. Overall, it must be noted that these studies gave no clear conclusion on the amounts or percentages of uptake. Hence, more research is needed to the extent and the exact mechanism of uptake. 
Table 2. Summary of the uptake of individual AGEs in different in vitro studies.

\begin{tabular}{|c|c|c|c|}
\hline Compound & Evidence & Notes & Reference(s) \\
\hline CML & $\begin{array}{l}\text { Strongly retained } \\
\text { inside Caco-2 cells. }\end{array}$ & $\begin{array}{l}\text { Diffused into gastrointestinal } \\
\text { epithelial (Caco-2) cells, but was } \\
\text { not able to cross the basolateral } \\
\text { membrane. Accumulation } \\
\text { in instestinal cells. Not likely } \\
\text { transported by amino acid } \\
\text { and peptide carriers and the } \\
\text { transepithelial flux measured } \\
\text { for the compounds occurs most } \\
\text { probably by simple diffusion. }\end{array}$ & {$[66,67]$} \\
\hline CEL & $\begin{array}{l}\text { Strongly retained } \\
\text { inside Caco-2 cells. }\end{array}$ & - & [66] \\
\hline MG-H1 & $\begin{array}{l}\text { Strongly retained } \\
\text { inside Caco-2 cells. }\end{array}$ & - & [66] \\
\hline Maltosine & $\begin{array}{l}\text { Absorbed as dipeptide } \\
\text { into Caco-2 cells by } \\
\text { PEPT1 and strongly } \\
\text { retained in cells. Not } \\
\text { absorbed in free form. }\end{array}$ & $\begin{array}{l}\text { Free maltosine permeates the } \\
\text { basolateral cell membrane } \\
\text { by simple diffusion down } \\
\text { its concentration gradient } \\
\text { and possibly by the action } \\
\text { of basolateral amino acid } \\
\text { transporters. }\end{array}$ & {$[66,68]$} \\
\hline $\begin{array}{l}\text { Glycated } \\
\text { dipeptides }\end{array}$ & $\begin{array}{l}\text { Absorbed into Caco-2 } \\
\text { cells by PEPT1. }\end{array}$ & $\begin{array}{l}\text { Intracellularly hydrolyzed by } \\
\text { peptidases to the free modified } \\
\text { amino acids and alanine. }\end{array}$ & {$[66]$} \\
\hline Pyrraline & $\begin{array}{l}\text { Not free pyrraline, but } \\
\text { the dipeptide with } \\
\text { alanine is absorbed } \\
\text { by PEPT1 in HeLa cells } \\
\text { (cervical cancer cells). }\end{array}$ & $\begin{array}{l}\text { After intracellular hydrolyzation } \\
\text { free pyrraline diffused through } \\
\text { the basolateral membrane. }\end{array}$ & {$[66,69]$} \\
\hline Fructoselysine & $\begin{array}{l}\text { Simple diffusion to a } \\
\text { small extent in Caco-2 } \\
\text { cells. }\end{array}$ & $\begin{array}{l}\text { Not likely transported by amino } \\
\text { acid and peptide carriers and the } \\
\text { transepithelial flux measured } \\
\text { for the compounds occurs most } \\
\text { probably by simple diffusion. }\end{array}$ & [67] \\
\hline
\end{tabular}




\section{The gastrointestinal tract}

\subsection{Physiology}

Based on the evidence regarding absorption of dAGEs, we suggest that the potential local effect of dAGEs in the GI tract may be of larger importance than that of dAGEs ending up systemically. The human digestive system consists of multiple parts, which are responsible for the mechanical and chemical digestion of food [75, 76]. Mechanical digestion starts in the mouth with the chewing (mastication) of food products to small boluses, which are then swallowed and enter the stomach through the oesophagus. From there until the rectum, mechanic digestion occurs through the peristaltic movements of the GI tract. Transport of the digested food product is also aided by the mucus layer covering the complete GI tract, which provides a barrier between the epithelial layer and the contents of the lumen. Chemical digestion occurs through different mechanisms. In the mouth, saliva contains salivary amylase, which starts the digestion of starch. Through the oesophagus, the food product then enters the stomach, in which it is mixed with stomach juice containing hydrochloric acid and has a $\mathrm{pH}$ of 2 . This low $\mathrm{pH}$ causes protein denaturation ensuring better access of pepsin (also secreted in the gastric juice) to the proteins. Pepsin hydrolyses proteins into peptides. After about one hour, the food product, now reduced to chyme, starts to pass the pyloric sphincter and travels further into the small intestine, which consists of the duodenum, jejunum, and ileum. The gradual transfer of chyme from the stomach to the duodenum can take up to two hours. In the duodenum, pancreatic juice containing pancreatic enzymes and sodium bicarbonate are added to the chyme. The sodium bicarbonate neutralizes the acidic chyme, while pancreatic enzymes hydrolyse carbohydrates, lipids, and proteins. Bile from the liver is also added to the chyme in the duodenum and acts as an emulsifier to bring fats into suspension, to ensure that pancreatic lipase can digest the lipids. In the jejunum and ileum, this digestion process continues and is aided by peristalsis. After the small intestine, the food products enter the large intestine: the colon. In the colon, water and electrolytes are absorbed from the chyme and colonic bacteria in the gut flora further degrade and ferment the macroand micronutrients $[75,76]$. The intestinal microbiota includes over 800 species of bacteria and additionally contains fungi, protozoa, and archaea [77]. This collective genome is called the gut microbiome. Some important functions of the microbiota include the breakdown of undigested fiber (polysaccharides) into short-chain fatty acids (SCFA) and the production of vitamin $\mathrm{K}$ and biotin. Dysbiosis of the normal intestinal microbiota is associated with different diseases such as inflammatory bowel diseases (IBD), obesity, autoimmune diseases and diabetes [78]. Diet has a large influence on the intestinal microbiota composition and may therefore aid in the treatment of these diseases.

\subsection{Digestion of dAGEs}

To what extent dAGEs are digested in the GI tract is currently unclear. Studies into the effect of gastric digestion on dAGEs are scarce and most studies are conducted using static in vitro models. In these models food products are most commonly exposed for two hours 
to synthetic gastric juice and pepsin and is set to a $\mathrm{pH}$ value of 2-2.5. However, as explained before, human gastric digestion is not a static environment; it is dynamic and includes peristaltic mixing and alternating $\mathrm{pH}$ levels [79]. The physiological $\mathrm{pH}$ levels in the stomach differ between 1-1.5 for basal levels and 5-7 immediately after ingestion. After approximately three hours after ingestion, the $\mathrm{pH}$ returns to basal levels [80]. Additionally, digestive fluids and meal components are removed from the GI tract by both passive and active absorption [79]. Unfortunately, the static in vitro models do not take such physiological parameters into account. In one of the existing studies on dAGE digestion, Hellwig et al. (2013) exposed casein modified with $\mathrm{N} \varepsilon$-fructoselysine, $\mathrm{CML}$, and lysinoalanine to synthetic gastric juice and pepsin with a $\mathrm{pH}$ value of 2 at $37^{\circ} \mathrm{C}$ for two hours. In this study, modifying casein led to a reduction of digestibility of casein in the intestines [81]. Although most of the physical parameters were taken into account, all dAGEs were exposed to the gastric juices for the complete two hours. This is not the case in the human body where constantly a small portion of the meal is transported to the duodenum and the gastric emptying time is depending on the type of food $[82,83]$.

\subsection{Colonic degradation}

More research into the digestion of protein-bound dAGEs is therefore necessary. In case they are not degraded in the GI tract, dAGEs may be further transported into the colon where they come into contact with the human colonic microbiota. Based on the available literature, the human colonic microbiota seems to be capable of only fermenting LMW dAGEs, and not the more complex HMW structures, including dAGEs and melanoidins. Xu et al. (2019) subjected glyoxal-glycated casein to static in vitro digestion and assessed the capability of human colonic microbiota to ferment these digested compounds [84]. Their results showed that human colonic microbiota was indeed able to ferment the LMW structures to a better extent than HMW structures. Although in a different study bifidobacteria have been shown to be capable of using bread melanoidins as carbon source, indicating prebiotic potential of bread melanoidins. However, the melanoidins were not digested in a digestion model prior to microbial exposure [85]. For the LMW weight structures, additional evidence can be found on the utilization of Amadori products, or early glycation products, by colonic microbiota. Human intestinal microbiota contain deglycating enzyme systems for Amadori products. For instance, E. coli has genes encoding for fructosamine kinase, which catalyses the production of the stable intermediate fructosamine-6-phosphate, and fructosamine-6-phosphate deglycase that in turn cleaves fructosamine-6-phosphate to glucose 6-phosphate and the corresponding amines [86]. Additionally, E. coli has been shown to utilize fructoselysine and a human gut commensal (Intestinimonas AF211) can produce butyrate from lysine and fructoselysine [87, 88]. Amadori products can furthermore be degraded to 1,2-dicarbonyl compounds by colonic bacteria, which are highly reactive with lysine $\varepsilon$-amino groups and arginine guanidine groups to form AGEs within the colon [89]. 


\subsection{Inflammation in the gastrointestinal tract}

Inflammatory bowel diseases (IBD) is the most commonly used term for a group of chronic inflammatory conditions of the GI tract, of which ulcerative colitis (UC) and Crohn's disease (CD) are the two main types. As the name indicates, these diseases present themselves through inflammation in the GI tract. In CD this inflammation may affect any segment of the GI tract from the mouth to the anus, while inflammatory patches in UC are limited to the colon [90]. Over the past years, these inflammatory diseases have emerged as a public health challenge. Prevalence of IBD has been increasing substantially over the last decades. In 2017, approximately 6-8 million people suffered from IBD [91]. Although the aetiology of IBD has been extensively investigated, the exact pathogenesis is still not fully known. Multiple factors are involved in the pathogenesis of IBD, such as environmental factors, immunological factors, genetic susceptibility, and diet [92]. Not only in the Western world, but also in newly industrialised parts of the world such as Brazil and Taiwan, the incidence of IBD is increasing [93]. This suggests the important role of the environment in the development of the disease. More specifically, the introduction of the Western diet in those areas has been suggested as a potential explanation of increased IBD incidence $[91,94]$. Treatment options of IBD include the use of glucocorticoids, which are very effective anti-inflammatory drugs. However, some people are resistant to glucocorticoids. This specific glucocorticoid resistance occurs in approximately $20 \%$ of people with IBD $[95,96]$. IBD patients often report the influence of nutrition on their symptoms, either worsening of relieving them, which indicates a possible role for nutrition in either the onset or treatment of the diseases [94].

Several studies exist that investigated the association between dAGE consumption and IBD. Elevated levels of the dAGE pentosidine were found in several cell types within the bowel of IBD patients [97]. It was suggested that the level of pentosidine reflects the extent of tissue damage, since it correlated with the expression of $8-\mathrm{OHdG}$, a marker for oxidative DNA damage. However, the pentosidine levels were correlated with age in $C D$, but not UC, indicating an association between pentosidine levels and tissue damage in UC and not in CD. Additionally the pentosidine levels were measured using an ELISA technique, which is not the most reliable technique for measuring AGEs. Andrassy et al. (2006) showed presence of carboxymethylated (glycated) endogenous S100 proteins in the inflamed tissue of IBD patients compared to non-inflamed tissue of the same patients [98]. Additionally, other clinical studies show evidence of a role of RAGE in IBD inflammation: in CD, protein levels of RAGE are upregulated in inflamed areas and thereby appear to play a role in the mechanisms involved in chronic inflammation. Similarly, in inflamed areas of the ileum and colon in UC, RAGE may play a role in sustaining and worsening the inflammation [99-101]. The gut endothelium in IBD patients furthermore shows a strong increase in both RAGE and NF-KB expression [20]. SRAGE, the soluble version of RAGE, is able to bind RAGE and thereby blocking the receptor. In CD patients, but not in UC patients, serum levels of SRAGE were found to be inversely correlated with C-reactive protein (CRP) levels and clinical activity index [102]. Moreover, different polymorphisms of RAGE were found to be associated with IBD occurrence in 
different patient groups, of which at least one polymorphism significantly increased RAGE transcriptional activation $[103,104]$. These results indicate a potential involvement of AGEs, via RAGE, in the inflammatory processes underlying IBD.

In addition to the aforementioned human studies, several animal studies also found interplays between IBD, dAGEs, and RAGE. By exposing C57BL/6 wild-type (WT) and RAGE-/- mice to $\mathrm{CML}$-modified-proteins from human inflamed intestinal tissue in the colon, Andrassy et al. (2006) showed a higher IL-6 expression in WT mice compared to RAGE-/- mice, indicating an essential role of RAGE in the intestinal inflammation [98]. Rodent studies into IBD usually involve indomethacin-induced enteritis and dextran sulfate sodium (DSS)- and 2,4,6-trinitrobenzene sulfonic acid (TNBS)-induced colitis to mimic IBD disease status. A recent study by BodyMalapel et al. (2019) showed that RAGE-/- mice were protected from indomethacin-induced enteritis and DSS- and TNBS-induced colitis, as iNOS expression was decreased in RAGE -/- mice compared to WT. This study concluded that reducing dAGEs may be a promising therapeutic target for IBD [101].Some animal studies have been conducted into IBD and an interplay with $d A G E s$, irrespective of investigating the role of RAGE. When giving healthy rats a heated diet that consisted of nine-fold higher dAGE levels than the normal diet, an increase in colonic oxidative stress and inflammation was seen [105]. Comparing a 'bread crust diet' to a normal diet, Yuan et al. (2018) found accumulation of dietary CML in intestinal tissue in rats, with the highest accumulation in the ileum [106]. The authors attributed this accumulation to the trapping of CML in the intestinal cell, as was seen earlier in the absorption studies by Hellwig et al. (2011) and Grunwald et al. (2006) [89, 107]. Additionally, a significant decrease in the activities of several antioxidant enzymes: superoxide dismutase (SOD) and glutathione peroxidase (GSH-PX), and a significant increase in TNF- $\alpha$ and IL-8 secretion were found in the duodenum compared to control group [106]. In a different study by Qu et al. (2017), rats were exposed to a regular (low-AGE) laboratory standard diet or heated (high-AGE) AIN$93 \mathrm{G}$ diet for 6, 12, and 18 weeks. Histological examination of the colon showed distorted crypts, crypt loss, goblet cell depletion, increased dysplasia, loose cellular arrangements, and thickening of the mucosa with oedema in rats exposed to the high-AGE diet. However, no increased inflammatory infiltration was present. The 18-week exposure group showed significantly decreased expression levels of the tight junction proteins Occludin and zonula occludens-1. All these results indicate an increased colon permeability due to increased dAGE intake [108]. NF-KB is known to have a high activity in the GI tract of IBD patients [109]. Interestingly, transgenic mice expressing firefly luciferase under the control of an NF-KB responsive promoter showed NF-KB activation in particularly the gut after ingestion of dAGES [110]. Remarkably, in an induced UC mice model, highly heated pellets reduced inflammation in the gut [111]. It has to be noted that this study focussed on melanoidins rather than dAGEs. In this study, Anton et al. (2012) exposed mice to standard, mildly heated, or highly heated pellets, after which a reduction in histopathological damage scores in mice fed highly heated pellets compared to mildly heated and standard pellets were found. However, the differences in $\mathrm{CML}$ content of the mildly heated and highly heated pellets were marginal, whereas the 
melanoidin content was almost 3 times higher for the highly heated pellets. The protective effect of the diets was therefore probably due to the melanoidin content and not the dAGE content [111]. A follow-up study showed less mast cell infiltration in DSS treated mice when administered a highly heated diet. However, in this study the diet was not analysed on MRP content and the treatment of the chow was different than in the aforementioned study. It is likely the effects were again caused by the melanoidin content, but it is not possible to attribute the effects to a specific subset of the MR [112]. Additionally, ALJahdali et al. (2017) administered CML to mice for 3 weeks and then induced colitis in two different subgroups with either DSS or TNBS [113]. They found no inflammation caused by only CML, which can be explained by the fact that CML was administered in a non-protein bound form. They also stated that CML failed to prevent an inflammatory response, but the article did not include clear reasoning on why an anti-inflammatory effect was expected. It is unclear whether CML administration continued during colitis induction. CML did limit DSS-induced weight loss, but not TNBS-induced weight loss. They also saw a pattern towards the control state in the gut microbiota in CML-DSS exposed mice compared to only DSS exposed mice.

From the results of the studies investigating the interplay between IBD, RAGE, and dAGEs it can be concluded that RAGE is involved in the symptoms and possibly in the disease course of IBD. Making the food-derived natural ligands (dAGEs) an interesting therapeutic target. Next to IBD, another gastrointestinal disease which symptoms seems to be largely influenced by nutrition is irritable bowel syndrome (IBS). IBS comprises of a group of symptoms that include abdominal pain and changes in the patterns of bowel movements. Symptoms often also include diarrhoea and constipation. Approximately $45 \%$ of the global population is affected by IBS. The cause and disease mechanism of IBS is unknown and there is no known cure. Therefore, treatment is focussed on symptom reduction. These treatments include medication to stimulate bowel movements and often dietary changes such as increasing soluble fibre intake [114]. 


\section{Aims and outline of the thesis}

In contrast to the multitude of studies into the effects of endogenous AGEs, dAGEs have been less well studied and several questions about these dAGEs remain. It is for instance still disputed to what extent and in what form dAGEs are absorbed. Independent of absorption, dAGEs enter the human GI tract after ingestion and it is also not clear what the local effects of dAGEs in the GI tract are. To obtain better insights into the possible effects of dAGEs in the GI tract, we performed several studies on the different effects that might occur after the ingestion of $d A G E s$. The overall aim of this thesis was to get an insight on the possible pro-inflammatory aspects of dAGEs in the GI tract. To have a first indication on the proinflammatory potential of dAGEs, and to stay as close to the properties of food products as possible, we created a glycated casein model based on milk powder in chapter $\mathbf{2}$. This mixture is then assessed for its potential induction of TNF- $\alpha$ secretion in human macrophagelike cells. Prior to entering the GI tract, dAGEs are ingested and digested by the human digestive system. To assess the effect of digestion on the dAGE-protein binding, in chapter $\mathbf{3}$ we use the sophisticated TNO gastrolntestinal Model (TIM-1) to analyse the breakdown of the dAGE-protein binding in both our glycated casein model and different food products. To what extent the digestion influences the pro-inflammatory characteristics of dAGEs on both macrophages and gastrointestinal epithelial cells is analysed in chapter $\mathbf{4}$. Glucocorticoids are among the most used anti-inflammatory drugs, the potential of these drugs to reduce dAGEinduced inflammation is researched and described in chapter $\mathbf{5}$. We show that dAGE-induced inflammation can cause glucocorticoid resistance in human macrophage-like cells, treating the cells with the antioxidant quercetin restores glucocorticoid sensitivity. Finally, all results, as well as its implications and limitations, are discussed in chapter 6. 


\section{References}

1. Walsh, J., Vladimir Putin employs a full-time food taster to ensure his meals aren't poisoned, in The Independent. 2014.

2. Agodi, A., et al., Association of Dietary Patterns with Metabolic Syndrome: Results from the Kardiovize Brno 2030 Study. Nutrients, 2018. 10(7).

3. Poulsen, M.W., et al., Advanced glycation endproducts in food and their effects on health. Food Chem Toxicol, 2013. 60: p. 10-37.

4. Hofmann, T., et al., Determination of the molecular weight distribution of non-enzymatic browning products formed by roasting of glucose and glycine and studies on their effects on NADPH-cytochrome c-reductase and glutathione-S-transferase in Caco-2 cells. Nahrung-Food, 2001. 45(3): p. 189-194.

5. Lin, J.A., C.H. Wu, and G.C. Yen, Perspective of Advanced Glycation End Products on Human Health. J Agric Food Chem, 2018. 66(9): p. 2065-2070.

6. Luevano-Contreras, C. and K. Chapman-Novakofski, Dietary advanced glycation end products and aging. Nutrients, 2010. 2(12): p. 1247-65.

7. Lorenzi, M., The polyol pathway as a mechanism for diabetic retinopathy: attractive, elusive, and resilient. Exp Diabetes Res, 2007. 2007: p. 61038.

8. Hofmann, T., Studies on the relationship between molecular weight and the color potency of fractions obtained by thermal treatment of glucose amino acid and glucose/protein solutions by using ultracentrifugation and color dilution techniques. Journal of Agricultural and Food Chemistry, 1998. 46(10): p. 3891-3895.

9. Oliver, C.M., L.D. Melton, and R.A. Stanley, Creating proteins with novel functionality via the Maillard reaction: a review. Crit Rev Food Sci Nutr, 2006. 46(4): p. 337-50.

10. Munch, G., et al., Amino acid specificity of glycation and protein-AGE crosslinking reactivities determined with a dipeptide SPOT library. Nat Biotechnol, 1999. 17(10): p. 1006-10.

11. Pratt, C.W. and K. Cornely, Essential biochemistry. 2nd ed. 2013, Hoboken, N.J. Chichester: Wiley; John Wiley distributor. xxvii, 704 p.

12. S. H. Ashoor, J.B.Z., Maillard Browning of Common Amino Acids and Sugars. Journal of Food Science, 1984. 49: p. 1206-1207.

13. Ajandouz, E.H. and A. Puigserver, Nonenzymatic browning reaction of essential amino acids: effect of pH on caramelization and Maillard reaction kinetics. J Agric Food Chem, 1999. 47(5): p. 1786-93.

14. Kwak, E.J. and S.I. Lim, The effect of sugar, amino acid, metal ion, and $\mathrm{NaCl}$ on model Maillard reaction under pH control. Amino Acids, 2004. 27(1): p. 85-90.

15. Hemmler, D., et al., Insights into the Chemistry of Non-Enzymatic Browning Reactions in Different Ribose-Amino Acid Model Systems. Sci Rep, 2018. 8(1): p. 16879.

16. Joubran, Y., et al., Implications of the Maillard reaction on bovine alpha-lactalbumin and its proteolysis during in vitro infant digestion. Food Funct, 2017. 8(6): p. 2295-2308.

17. Lund, M.N. and C.A. Ray, Control of Maillard Reactions in Foods: Strategies and Chemical Mechanisms. J Agric Food Chem, 2017. 65(23): p. 4537-4552.

18. Helou, C., et al., The impact of raw materials and baking conditions on Maillard reaction products, thiamine, folate, phytic acid and minerals in white bread. Food Funct, 2016. 7(6): p. 2498-507.

19. Milkovska-Stamenova, S. and R. Hoffmann, Influence of storage and heating on protein glycation levels of processed lactose-free and regular bovine milk products. Food Chem, 2017. 221: p. 489-495.

20. Prosser, C.G., E.A. Carpenter, and A.J. Hodgkinson, N(epsilon)-carboxymethyllysine in nutritional milk formulas for infants. Food Chem, 2019. 274: p. 886-890.

21. Delgado-Andrade, C. and V. Fogliano, Dietary Advanced Glycosylation End-Products (dAGEs) and Melanoidins Formed through the Maillard Reaction: Physiological Consequences of their Intake. Annu Rev Food Sci Technol, 2018. 9: p. 271-291.

22. Finot, P.A. and E. Magnenat, Metabolic transit of early and advanced Maillard products. Prog Food Nutr Sci, 1981. 5(1-6): p. 193-207.

23. Klenovics, K.S., et al., Advanced glycation end products in infant formulas do not contribute to insulin resistance associated with their consumption. PLoS One, 2013. 8(1): p. e53056. 
24. Oldfield, M.D., et al., Advanced glycation end products cause epithelial-myofibroblast transdifferentiation via the receptor for advanced glycation end products (RAGE). J Clin Invest, 2001. 108(12): p. 1853-63.

25. Yanagisawa, K., et al., Specific fluorescence assay for advanced glycation end products in blood and urine of diabetic patients. Metabolism, 1998. 47(11): p. 1348-53.

26. Wagner, Z., et al., N(epsilon)-(carboxymethyl)lysine levels in patients with type 2 diabetes: role of renal function. Am J Kidney Dis, 2001. 38(4): p. 785-91.

27. Goldberg, T., et al., Advanced glycoxidation end products in commonly consumed foods. J Am Diet Assoc, 2004. 104(8): p. 1287-91.

28. Hellwig, M., J. Degen, and T. Henle, 3-deoxygalactosone, a "new" 1,2-dicarbonyl compound in milk products. J Agric Food Chem, 2010. 58(19): p. 10752-60.

29. Helou, C., et al., Maillard reaction products in bread: A novel semi-quantitative method for evaluating melanoidins in bread. Food Chem, 2016. 190: p. 904-11.

30. Troise, A.D., Analytical strategies to depict the fate of the Maillard reaction in foods. Current Opinion in Food Science, 2018. 19: p. 15-22.

31. Hull, G.L.J., et al., N-epsilon-(carboxymethyl)lysine content of foods commonly consumed in a Western style diet. Food Chemistry, 2012. 131(1): p. 170-174.

32. Niquet-Leridon, C., et al., The rehabilitation of raw and brown butters by the measurement of two of the major Maillard products, N(epsilon)-carboxymethyl-lysine and

5-hydroxymethylfurfural, with validated chromatographic methods. Food Chem, 2015. 177: p. 361-8.

33. Scheijen, J., et al., Analysis of advanced glycation endproducts in selected food items by ultra-performance liquid chromatography tandem mass spectrometry: Presentation of a dietary AGE database. Food Chem, 2016. 190: p. 1145-1150.

34. Delatour, T., et al., Analysis of advanced glycation endproducts in dairy products by isotope dilution liquid chromatography-electrospray tandem mass spectrometry. The particular case of carboxymethyllysine. Journal of Chromatography A, 2009. 1216(12): p. 2371-2381.

35. Degen, J., M. Hellwig, and T. Henle, 1,2-dicarbonyl compounds in commonly consumed foods. J Agric Food Chem, 2012. 60(28): p. 7071-9.

36. Zhao, D., et al., Comparison of Free and Bound Advanced Glycation End Products in Food: A Review on the Possible Influence on Human Health. J Agric Food Chem, 2019. 67(51): p. 14007-14018.

37. Hegele, J., T. Buetler, and T. Delatour, Comparative LC-MS/MS profiling of free and proteinbound early and advanced glycation-induced lysine modifications in dairy products. Anal Chim Acta, 2008. 617(1-2): p. 85-96.

38. Henle, T., G. Zehetner, and H. Klostermeyer, Fast and sensitive determination of furosine. Z Lebensm Unters Forsch, 1995. 200(3): p. 235-7.

39. Kierdorf, K. and G. Fritz, RAGE regulation and signaling in inflammation and beyond. J Leukoc Biol, 2013. 94(1): p. 55-68.

40. Xie, J., et al., Cellular signalling of the receptor for advanced glycation end products (RAGE). Cell Signal, 2013. 25(11): p. 2185-97.

41. Chen, P.M., J.B. Zhao, and H. Gregersen, Up-Regulated Expression of Advanced Glycation End-Products and Their Receptor in the Small Intestine and Colon of Diabetic Rats. Digestive Diseases and Sciences, 2012. 57(1): p. 48-57.

42. Xue, J., et al., Advanced glycation end product recognition by the receptor for AGEs. Structure, 2011. 19(5): p. 722-32.

43. Xie, J., et al., Structural basis for pattern recognition by the receptor for advanced glycation end products (RAGE). J Biol Chem, 2008. 283(40): p. 27255-69.

44. Schmidt, A.M., et al., The biology of the receptor for advanced glycation end products and its ligands. Biochim Biophys Acta, 2000. 1498(2-3): p. 99-111.

45. Yao, D. and M. Brownlee, Hyperglycemia-induced reactive oxygen species increase expression of the receptor for advanced glycation end products (RAGE) and RAGE ligands. Diabetes, 2010. 59(1): p. 249-55.

46. Bongarzone, S., et al., Targeting the Receptor for Advanced Glycation Endproducts (RAGE): A Medicinal Chemistry Perspective. J Med Chem, 2017. 60(17): p. 7213-7232.

47. Bierhaus, A., et al., Understanding RAGE, the receptor for advanced glycation end products. J Mol Med (Berl), 2005. 83(11): p. 876-86. 
48. Zhou, Z., et al., Metformin Inhibits Advanced Glycation End Products-Induced Inflammatory Response in Murine Macrophages Partly through AMPK Activation and RAGE/NFkappaB Pathway Suppression. J Diabetes Res, 2016. 2016: p. 4847812.

49. Son, S., et al., Advanced glycation end products impair NLRP3 inflammasome-mediated innate immune responses in macrophages. J Biol Chem, 2017. 292(50): p. 20437-20448.

50. Cai, W., et al., Advanced glycation end product (AGE) receptor 1 suppresses cell oxidant stress and activation signaling via EGF receptor. Proc Natl Acad Sci U S A, 2006. 103(37): p. 13801-6.

51. Cai, W., et al., AGER1 regulates endothelial cell NADPH oxidase-dependent oxidant stress via PKC-delta: implications for vascular disease. Am J Physiol Cell Physiol, 2010. 298(3): p. C62434.

52. Lu, C., et al., Advanced glycation endproduct (AGE) receptor 1 is a negative regulator of the inflammatory response to AGE in mesangial cells. Proc Natl Acad Sci U S A, 2004. 101(32): p. 11767-72.

53. Vlassara, H., et al., Advanced glycation end product homeostasis- Exogenous oxidants and innate defenses. Maillard Reaction: Recent Advances in Food and Biomedical Sciences, 2008. 1126: p. 46-52.

54. Bansal, S., et al., Advanced glycation end products enhance reactive oxygen and nitrogen species generation in neutrophils in vitro. Mol Cell Biochem, 2012. 361(1-2): p. 289-96.

55. Wautier, M.P., et al., Activation of NADPH oxidase by AGE links oxidant stress to altered gene expression via RAGE. Am J Physiol Endocrinol Metab, 2001. 280(5): p. E685-94.

56. Rowan, S., E. Bejarano, and A. Taylor, Mechanistic targeting of advanced glycation endproducts in age-related diseases. Biochim Biophys Acta Mol Basis Dis, 2018. 1864(12): p. 3631-3643.

57. Nowotny, K., et al., Advanced glycation end products and oxidative stress in type 2 diabetes mellitus. Biomolecules, 2015. 5(1): p. 194-222.

58. Kalapos, M.P., The tandem of free radicals and methylglyoxal. Chem Biol Interact, 2008. 171(3): p. 251-71.

59. Van Puyvelde, K., et al., Effect of advanced glycation end product intake on inflammation and aging: a systematic review. Nutrition Reviews, 2014. 72(10): p. 638-650.

60. Hussain, M., et al., Novel insights in the dysfunction of human blood-brain barrier after glycation. Mech Ageing Dev, 2016. 155: p. 48-54.

61. Bucala, R., et al., Modification of Low-Density-Lipoprotein by Advanced Glycation EndProducts Contributes to the Dyslipidemia of Diabetes and Renal-Insufficiency. Proceedings of the National Academy of Sciences of the United States of America, 1994. 91(20): p. 94419445.

62. Liang, Z., et al., The fate of dietary advanced glycation end products in the body: from oral intake to excretion. Crit Rev Food Sci Nutr, 2019: p. 1-17.

63. Delgado-Andrade, C., Carboxymethyl-lysine: thirty years of investigation in the field of AGE formation. Food Funct, 2016. 7(1): p. 46-57.

64. Faist, V. and H.F. Erbersdobler, Metabolic transit and in vivo effects of melanoidins and precursor compounds deriving from the Maillard reaction. Annals of Nutrition and Metabolism, 2001. 45(1): p. 1-12.

65. Somoza, V., Five years of research on health risks and benefits of Maillard reaction products: an update. Mol Nutr Food Res, 2005. 49(7): p. 663-72.

66. Roncero-Ramos, I., et al., An Advanced Glycation End Product (AGE)-Rich Diet Promotes $\mathrm{N}$ epsilon-Carboxymethyl-lysine Accumulation in the Cardiac Tissue and Tendons of Rats. Journal of Agricultural and Food Chemistry, 2014. 62(25): p. 6001-6006.

67. $\mathrm{Xu}, \mathrm{H} . \mathrm{Z}$., et al., Biodistribution and Elimination Study of Fluorine-18 Labeled N-epsilonCarboxymethyl-Lysine following Intragastric and Intravenous Administration. Plos One, 2013. 8(3).

68. Degen, J., et al., Dietary influence on urinary excretion of 3-deoxyglucosone and its metabolite 3-deoxyfructose. J Agric Food Chem, 2014. 62(11): p. 2449-56.

69. Foerster, A. and T. Henle, Glycation in food and metabolic transit of dietary AGEs (advanced glycation end-products): studies on the urinary excretion of pyrraline. Biochemical Society Transactions, 2003. 31: p. 1383-1385.

70. FÖRster, A., Y. KÜHne, and T.O. Henle, Studies on Absorption and Elimination of Dietary 
Maillard Reaction Products. Annals of the New York Academy of Sciences, 2005. 1043(1): p. 474-481.

71. Uribarri, J., et al., Restriction of dietary glycotoxins reduces excessive advanced glycation end products in renal failure patients. J Am Soc Nephrol, 2003. 14(3): p. 728-31.

72. Sebekova, K., et al., Plasma concentration and urinary excretion of N-epsilon(carboxymethyl)lysine in breast milk- and formula-fed infants. Maillard Reaction: Recent Advances in Food and Biomedical Sciences, 2008. 1126: p. 177-180.

73. Davis, K.E., et al., Contribution of dietary advanced glycation end products (AGE) to circulating AGE: role of dietary fat. British Journal of Nutrition, 2015. 114(11): p. 1797-1806.

74. Scheijen, J., et al., Dietary intake of advanced glycation endproducts is associated with higher levels of advanced glycation endproducts in plasma and urine: The CODAM study. Clin Nutr, 2018. 37(3): p. 919-925.

75. E. Whitney, S.R.R., Understanding Nutrition. 11th ed. 2008, Belmont, CA, USA: Thomson Higher Education.

76. Fox, S.I., Human Physiology. 11th ed. 2009, New York, NY, USA: McGraw-Hill.

77. O'Keefe, S.J., Nutrition and colonic health: the critical role of the microbiota. Curr Opin Gastroenterol, 2008. 24(1): p. 51-8.

78. Xu, Z. and R. Knight, Dietary effects on human gut microbiome diversity. Br J Nutr, 2015. 113 Suppl: p. S1-5.

79. Guerra, A., et al., Relevance and challenges in modeling human gastric and small intestinal digestion. Trends in Biotechnology, 2012. 30(11): p. 591-600.

80. Sams, L., et al., Relevant pH and lipase for in vitro models of gastric digestion. Food Funct, 2016. 7(1): p. 30-45.

81. Hellwig, M., et al., N-epsilon-fructosyllysine and N-epsilon-carboxymethyllysine, but not Iysinoalanine, are available for absorption after simulated gastrointestinal digestion. Amino Acids, 2014. 46(2): p. 289-99.

82. Stanstrup, J., et al., Whey protein delays gastric emptying and suppresses plasma fatty acids and their metabolites compared to casein, gluten, and fish protein. J Proteome Res, 2014. 13(5): p. 2396-408.

83. Read, N.W., et al., Transit of a meal through the stomach, small intestine, and colon in normal subjects and its role in the pathogenesis of diarrhea. Gastroenterology, 1980. 79(6): p. $1276-82$.

84. Xu, D., et al., Degradation of Peptide-Bound Maillard Reaction Products in Gastrointestinal Digests of Glyoxal-Glycated Casein by Human Colonic Microbiota. J Agric Food Chem, 2019. 67(43): p. 12094-12104.

85. Borrelli, R.C. and V. Fogliano, Bread crust melanoldins as potential prebiotic ingredients. Molecular Nutrition \& Food Research, 2005. 49(7): p. 673-678.

86. Deppe, V.M., et al., Enzymatic deglycation of Amadori products in bacteria: mechanisms, occurrence and physiological functions. Appl Microbiol Biotechnol, 2011. 90(2): p. 399-406.

87. Wiame, E., et al., Identification of a pathway for the utilization of the Amadori product fructoselysine in Escherichia coli. J Biol Chem, 2002. 277(45): p. 42523-9.

88. Bui, T.P.N., et al., Production of butyrate from lysine and the Amadori product fructoselysine by a human gut commensal. Nature Communications, 2015. 6.

89. Hellwig, M., et al., Transport of free and peptide-bound glycated amino acids: synthesis, transepithelial flux at Caco-2 cell monolayers, and interaction with apical membrane transport proteins. Chembiochem, 2011. 12(8): p. 1270-9.

90. Hendrickson, B.A., R. Gokhale, and J.H. Cho, Clinical aspects and pathophysiology of inflammatory bowel disease. Clin Microbiol Rev, 2002. 15(1): p. 79-94.

91. Collaborators, G.B.D.I.B.D., The global, regional, and national burden of inflammatory bowel disease in 195 countries and territories, 1990-2017: a systematic analysis for the Global Burden of Disease Study 2017. Lancet Gastroenterol Hepatol, 2020. 5(1): p. 17-30.

92. Khor, B., A. Gardet, and R.J. Xavier, Genetics and pathogenesis of inflammatory bowel disease. Nature, 2011. 474(7351): p. 307-17.

93. Ng, S.C., et al., Worldwide incidence and prevalence of inflammatory bowel disease in the 21st century: a systematic review of population-based studies. Lancet, 2018. 390(10114): p. 2769-2778.

94. Rizzello, F., et al., Implications of the Westernized Diet in the Onset and Progression of IBD. 
Nutrients, 2019. 11(5).

95. Tung, J., et al., A population-based study of the frequency of corticosteroid resistance and dependence in pediatric patients with Crohn's disease and ulcerative colitis. Inflamm Bowel Dis, 2006. 12(12): p. 1093-100.

96. Munkholm, P., et al., Frequency of glucocorticoid resistance and dependency in Crohn's disease. Gut, 1994. 35(3): p. 360-2.

97. Kato, S., et al., Increased pentosidine, an advanced glycation end-product, in urine and tissue reflects disease activity in inflammatory bowel diseases. Journal of Gastroenterology and Hepatology, 2008. 23: p. S140-S145.

98. Andrassy, M., et al., Posttranslationally modified proteins as mediators of sustained intestinal inflammation. American Journal of Pathology, 2006. 169(4): p. 1223-1237.

99. Ciccocioppo, R., et al., Role of the advanced glycation end products receptor in Crohn's disease inflammation. World Journal of Gastroenterology, 2013. 19(45): p. 8269-8281.

100. Zen, K., et al., Receptor for advanced glycation endproducts mediates neutrophil migration across intestinal epithelium. Journal of Immunology, 2007. 178(4): p. 2483-2490.

101. Body-Malapel, M., et al., The RAGE signaling pathway is involved in intestinal inflammation and represents a promising therapeutic target for Inflammatory Bowel Diseases. Mucosal Immunol, 2019. 12(2): p. 468-478.

102. Ciccocioppo, R., et al., The Circulating Level of Soluble Receptor for Advanced Glycation End Products Displays Different Patterns in Ulcerative Colitis and Crohn's Disease: A Cross-Sectional Study. Dig Dis Sci, 2015. 60(8): p. 2327-37.

103. Ciccocioppo, R., et al., Functional polymorphisms of the receptor for the advanced glycation end product promoter gene in inflammatory bowel disease: a case-control study. Clin Exp Med, 2019. 19(3): p. 367-375.

104. Dabritz, J., et al., The functional-374T/A polymorphism of the receptor for advanced glycation end products may modulate Crohn's disease. Am J Physiol Gastrointest Liver Physiol, 2011. 300(5): p. G823-32.

105. Shangari, N., et al., A thermolyzed diet increases oxidative stress, plasma alpha-aldehydes and colonic inflammation in the rat. Chemico-Biological Interactions, 2007. 169(2): p. 100-109.

106. Yuan, X.J., et al., Accumulation and effects of dietary advanced glycation end products on the gastrointestinal tract in rats. International Journal of Food Science and Technology, 2018. 53(10): p. 2273-2281.

107. Grunwald, S., et al., Transepithelial flux of early and advanced glycation compounds across Caco- 2 cell monolayers and their interaction with intestinal amino acid and peptide transport systems. Br J Nutr, 2006. 95(6): p. 1221-8.

108. Qu, W., et al., Dietary advanced glycation end products modify gut microbial composition and partially increase colon permeability in rats. Mol Nutr Food Res, 2017. 61(10).

109. Rogler, G., et al., Nuclear factor kappaB is activated in macrophages and epithelial cells of inflamed intestinal mucosa. Gastroenterology, 1998. 115(2): p. 357-69.

110. Nass, N., K. Bayreuther, and A. Simm, Systemic activation of NF-kappaB driven luciferase activity in transgenic mice fed advanced glycation end products modified albumin. Glycoconj J, 2017. 34(2): p. 157-161.

111. Anton, P.M., et al., Highly heated food rich in Maillard reaction products limit an experimental colitis in mice. Food \& Function, 2012. 3(9): p. 941-949.

112. Al Amir, I., et al., Maillard reaction products from highly heated food prevent mast cell number increase and inflammation in a mouse model of colitis. Nutr Res, 2017. 48: p. 26-32.

113. N, A., et al., Repeated Oral Exposure to N (epsilon)-Carboxymethyllysine, a Maillard Reaction Product, Alleviates Gut Microbiota Dysbiosis in Colitic Mice. Dig Dis Sci, 2017. 62(12): p. 3370-3384.

114. Thuisarts.nl. Ik heb een prikkelbare darm. 2017 [cited 2020 23-02-2020]; Available from: https://www.thuisarts.nl/prikkelbare-darm/ik-heb-prikkelbare-darm\#medicijnenbij-een-prikkelbare-darm. 

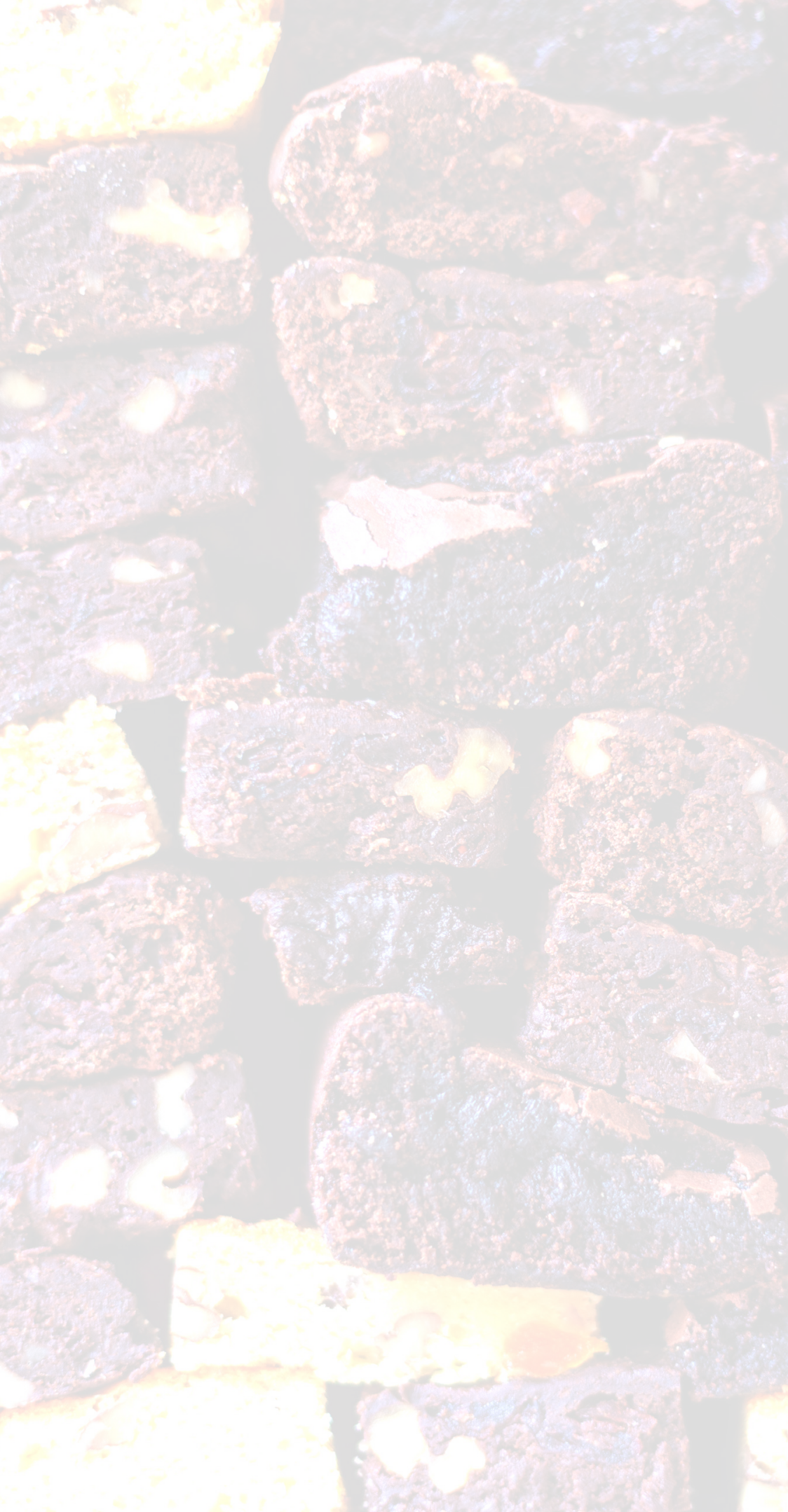


\section{Chapter 2}

\section{Dietary advanced glycation endproducts induce an inflammatory response in human macrophages in vitro}

Timme van der Lugt, Antje R. Weseler, Wouter A. Gebbink, Misha F. Vrolijk, Antoon Opperhuizen, Aalt Bast.

Nutrients, 2018 Dec; 10(12): 1868 


\section{Abstract}

Advanced glycation endproducts (AGEs) can be found in protein- and sugar-rich food products processed at high temperatures, which make up a vast amount of the Western diet. The effect of AGE-rich food products on human health is not yet clear and controversy still exists due to possible contamination of samples with endotoxin and the use of endogenous formed AGEs. AGEs occur in food products, both as protein-bound and individual molecules. Which form exactly induces a pro-inflammatory effect is also unknown. In this study, we exposed human macrophage-like cells to dietary AGEs, both in a protein matrix and individual AGEs. It was ensured that all samples did not contain endotoxin concentrations $>0.06 \mathrm{EU} / \mathrm{mL}$. The dietary AGEs induced TNF- $\alpha$ secretion of human macrophage-like cells. This effect was decreased by the addition of $\mathrm{N} \varepsilon$-carboxymethyllysine $(\mathrm{CML})$-antibodies or a receptor for advanced glycation endproducts (RAGE) antagonist. None of the individual AGEs induce any TNF- $\alpha$, indicating that AGEs should be bound to proteins to exert an inflammatory reaction. These findings show that dietary AGEs directly stimulate the inflammatory response of human innate immune cells and help us define the risk of regular consumption of AGE-rich food products on human health. 


\section{Introduction}

During heating of food products containing protein and carbohydrates, dietary advanced glycation endproducts (AGEs) are formed via the Maillard reaction [1]. AGEs are greatly responsible for the flavor and taste of food, and increase palatability. In the Maillard reaction, the carbonyl group of a reducing sugar (sugars with a free anomeric carbon [2]) reacts with an amino moiety of an amino acid leading to reactive intermediates, the so-called Amadori products. These Amadori products then form different AGEs [3]. AGEs comprise a large body of different molecules [3]. Examples of well-studied AGEs are: $\mathrm{N} \varepsilon$-carboxymethyllysine (CML), carboxyethyllysine (CEL), methylglyoxal-derived hydroimidazolone (MG-H1), pentosidine, and acrylamide. Although the quantification of AGEs in food products is frequently done by antibody-based techniques [4,5], liquid chromatography-tandem mass spectrometry (LC-MS/MS) is the preferred technique to accurately identify and quantify individual AGEs $[1,6-8]$. Physiological consequences of exposure to dietary AGEs are not yet fully understood. Endogenously formed AGEs in diabetic patients, however, have been shown to induce an inflammatory reaction and contribute to the onset of cardiovascular diseases, such as atherosclerosis and diabetic cardiomyopathy [9]. The inflammatory effect seen in these situations is proposed to be caused by the binding of AGEs to the specific receptor for advanced glycation endproducts (RAGE). RAGE activates many enzymes and protein complexes, one of which is nuclear factor kappa-light-chain-enhancer of activated B cells (NF-KB) [10]. Even though inflammation caused by endogenous AGEs has been thoroughly studied, information on the generation of inflammation by dietary AGEs is scarce. As said, many of the studies on the effects of AGEs are conducted with endogenously formed AGEs, by heating bovine serum albumin or human serum albumin with different sugars at $37^{\circ} \mathrm{C}$ for several hours, mimicking the endogenous situation. This difference with a home cooking situation, in which dietary proteins and sugars are heated to higher temperatures, can lead to a different array of molecules, resulting in different immunological outcomes. In addition to endogenous AGEs, some individual AGEs have been researched. Acrylamide is a well-studied compound, but only with regards to its genotoxic and carcinogenic effects $[11,12]$. To our knowledge, no study has been published on the inflammatory effect of acrylamide. A clear cause-effect relationship between AGEs and inflammation has also not be established yet and the available results have been questioned as being caused by endotoxins rather than AGEs $[13,14]$. To our knowledge, we are the first to investigate the inflammatory effect of dietary AGEs in a home-cooking environment and the first to investigate the inflammatory effect of acrylamide. The present study aimed at investigating the effects of dietary AGEs on the inflammatory response of human cells of the innate immune system. To mimic a home cooking situation, only dietary protein and sugars were used and heated to a temperature of $100^{\circ} \mathrm{C}$. The formation of dietary AGEs over time was quantified by LC-MS/MS and the presence of endotoxin was excluded. We also examined the effect of individual AGEs and whether the observed inflammatory effect was induced by activation of RAGE. 


\section{Materials and Methods}

\subsection{Chemicals and Reagents}

Casein from bovine milk, $\alpha$-lactose monohydrate, $\mathrm{NaOH}$, sodium-phospate, 2-mercaptoethanol, and thiazolyl blue tetrazolium bromide (MTT) were obtained from SigmaAldrich (Saint Louis, MO USA). D-glucose, glutamate, fetal bovine serum (FBS), Dulbeccco's Phospate-Buffered Saline (DPBS) were obtained from Gibco (Thermo Scientific, Waltham, MA, USA). Analytical standards of CML (>99\%), CEL (>95\%), MG-H1 (>93\%), and pentosidine (>99\%), as well as the deuterium labelled internal standards CML-d2, CEL-d4, and MG-H1-d3, were obtained from Polypeptide (Strasbourg, France). Boric acid (99.5\%), chloroform (99.5\%), nonafluoropentanoic acid (NFPA; 99\%), sodium hydroxide (98\%), sodium borohydride (96\%), trifluoroacetic acid (TFA; 99\%), and phorbol 12-myristate 13-acetate (PMA) were obtained from Sigma (Zwijndrecht, Netherlands). HPLC-grade acetonitrile and methanol were obtained from Actu-all Chemicals (Oss, Netherlands).

\subsection{Preparation of Glycated Casein}

Casein, glucose, and lactose, in the proportions of milk powder (11 mM glucose, $0.2 \mathrm{M}$ lactose, $10 \mathrm{~g} / \mathrm{L}$ casein from bovine milk), were diluted in $50 \mathrm{mM}$ phosphate buffer, $\mathrm{pH} 7.4$ in and heated in an Erlenmeyer on a heating plate at $100^{\circ} \mathrm{C}$ up to $120 \mathrm{~min}$. At time points 0,15 , 30, 60, 90 and $120 \mathrm{~min}$. samples were taken and immediately cooled in ice water. Samples were aliquoted and stored at $-80^{\circ} \mathrm{C}$.

\subsection{Determination of Endotoxin in Glycated Casein}

The presence of endotoxin in the glycated casein samples from all time points was assessed by the commercially available PYROGENT Gel Clot LAL Assay 0.06 EU/mL sensitivity (Lonza, Basel, Switzerland). The assay was performed in accordance with the manufacturer's protocol using endotoxin-free dilutions and reagent tubes (Lonza, Basel, Switzerland).

\subsection{Assessment of AGE-Formation by Fluorescence Spectroscopy}

AGE-formation was monitored by fluorometry measuring AGE-specific fluorescence. $100 \mu \mathrm{L}$ of each sample was added in duplicate in a clear bottom black polystyrene microplate (Corning, Corning, NY, USA) and read in a microplate reader (Spectramax M2 Multi-Mode, Molecular Devices, San Jose, CA, USA) at an excitation wavelength of $\lambda$ ex $=370 \mathrm{~nm}$ and an emission wavelength of $\lambda e m=440 \mathrm{~nm}[14]$.

\subsection{Quantification of AGEs in Glycated Casein by UPLC-Tandem MS/MS}

Sample preparation is based on published methods $[6,15]$. Briefly, sodium borate $(0.33 \mathrm{M}$; $\mathrm{pH}$ 9) and sodium borohydride (2 M) were added to an aliquot of the samples (equivalent to $\sim 1 \mathrm{mg}$ protein) to obtain a sodium borohydride concentration of $0.2 \mathrm{M}$ in the samples. These sample solutions were incubated for $4 \mathrm{~h}$ at room temperature. Subsequently, $1 \mathrm{~mL}$ of a chloroform/methanol $(2 / 1(\mathrm{v} / \mathrm{v}))$ mix was added, and the samples were centrifuged for $10 \mathrm{~min}$ 
at $12,000 \mathrm{rpm}$. The chloroform phase was discarded and the remaining solution containing proteins was hydrolyzed in $6 \mathrm{M} \mathrm{HCl}$ at $110^{\circ} \mathrm{C}$ for $18 \mathrm{~h}$. The solutions were evaporated until dryness at $50{ }^{\circ} \mathrm{C}$ under a stream of nitrogen and dissolved in $1 \%(\mathrm{v} / \mathrm{V})$ aqueous TFA $(1 \mathrm{~mL})$. Prior to UPLC-MS/MS analysis, internal standards and MilliQ were added to $50 \mu \mathrm{L}$ of these analyte solutions to achieve a final volume of $200 \mu \mathrm{L}$. The samples were analyzed using an Acquity UPLC system (Waters, Milford, MA, USA) equipped with a BEH C18 analytical column $(50 \times 2.1 \mathrm{~mm}, 1.7 \mu \mathrm{m}$ particle size, Waters, Milford, MA, USA)). The mobile phases were 5 mM NFPA in water (A) and acetonitrile (B), and the flow was set at $0.3 \mathrm{~mL} / \mathrm{min}$. The mobile phase gradient was as follows: a linear increase from $90 \% \mathrm{~A}$ ( $\mathrm{t}=0 \mathrm{~min}$.) to $50 \% \mathrm{~A}(\mathrm{t}=4$ min.) followed by a 1 min. equilibration at the initial conditions. The column was kept at a constant temperature of $45^{\circ} \mathrm{C}$. A Quattro Premier triple quadrupole mass spectrometer (Waters) was connected to the UPLC was operated in positive electrospray ionization (ESI+). The capillary voltage was set to $3.0 \mathrm{kV}$, and the source and desolvation temperatures were $120^{\circ} \mathrm{C}$ and $450^{\circ} \mathrm{C}$, respectively. The cone and desolvation gas flow were set at $100 \mathrm{~L} / \mathrm{h}$ and $600 \mathrm{~L} / \mathrm{h}$, respectively. Compound-specific cone voltages and collision energies can be found in Table S1. Quantification was performed using an internal standard approach and ninepoint calibration curves. MG-H1-d3 was used as internal standard for the quantification of pentosidine. Quantification was performed using the precursor-product ion multiple reaction monitoring (MRM) transitions reported in Table S1. Instrumental detection and quantitation limits (IDL and IQL, respectively) were determined as the concentration from a peak with a signal-to-noise ratio of 3 and 10, respectively, in a standard chromatogram. Method detection and quantitation limits (MDL and MQL, respectively) were determined for AGEs detected in sample extracts as the concentration from a peak with a signal-to-noise ratio of 3 and 10, respectively, in a sample chromatogram. For pentosidine (not detected in the samples) the MDL and MQL were determined using a value of 3 and 10 times the noise at the retention time of pentosidine as peak area. Compound-specific detection and quantification limits are listed in Table S2. The arithmetic mean recovery ( \pm SE) was $113 \pm 2 \%$ for CML-d2, $104 \pm 2 \%$ for CEL-d4, and $33 \pm 1 \%$ for MG-H1-d3. The relative differences observed between duplicate analyses were $12 \%, 7 \%$, and $15 \%$ for $\mathrm{CML}, \mathrm{CEL}$, and MG-H1, respectively.

\subsection{Cell Culture and Exposure}

THP-1 monocytes (ATCC, TIB- 202), cultured in RPMI 1640 with L-glutamine, Hepes and phenol red (Gibco, Thermo Scientific, Waltham, MA, USA) supplemented with $10 \%(\mathrm{v} / \mathrm{V})$ FBS, D-glucose $(4.5 \mathrm{~g} / \mathrm{L})$, Na-pyruvate $(1 \mathrm{mM})$ and 2-mercaptoethanol $(50 \mu \mathrm{M})$, were seeded in a 96-wells plate at a cell density of 70,000 cells/well and differentiated into macrophages by adding $200 \mathrm{nM}$ of PMA to the cell culture medium and culturing them for $72 \mathrm{~h}$. After differentiation, the cells were exposed to $1,2.5,5$ and $10 \%(\mathrm{~V} / \mathrm{V})$ of glycated casein in serum and phenol-red free medium and always in a 50\% DPBS solution. Only samples that were proven to be free of endotoxins were used. Control conditions included phenol-red free medium and 50\% DPBS. Incubations were done both with and without the anti-carboxymethyl 
Iysine (CML) antibody (Abcam, Cambridge, MA, USA) in a 1/500 dilution. Samples with anti-CML antibody were incubated overnight at $4^{\circ} \mathrm{C}$ before using. For the RAGE antagonist experiments, cells were incubated with $10 \%$ glycated casein and with and without 1 HM FPSZM1 (Merck, Darmstadt, Germany).

\subsection{Assessment of Cell Viability}

Cell viability was assessed using the MTT assay. Supernatant was removed and stored for ELISA analyses. $100 \mu \mathrm{l}$ of MTT solution $(0.5 \mathrm{mg} / \mathrm{mL}$ in DPBS) was added to each well, after which the plate was incubated in the dark at $37^{\circ} \mathrm{C}$ for $1 \mathrm{~h}$. After $1 \mathrm{~h}$, the MTT solution was removed from the wells and $100 \mu$ l of DMSO was added to each well. After 10 min incubation at room temperature, absorbance was measured at $\lambda=540 \mathrm{~nm}$ in a microplate reader (BioRad, Hercules, California, USA).

\subsection{Quantification of Tumor Necrosis Factor (TNF)- $\alpha$ Release by ELISA}

Tumor necrosis factor (TNF)- $\alpha$ release in cell culture supernatant was assessed by a commercially available ELISA kit (Sanquin, Amsterdam, the Netherlands). The analyses were carried out in accordance with the manufacturer's protocol using the Pelikine Toolset for all reagents (Sanquin). For the individual AGEs and RAGE experiments, TNF- $\alpha$ release was assessed by a commercially available ELISA kit (R\&D systems, Minneapolis, MN, USA). The analyses were carried out in accordance with the manufacturer's protocol.

\subsection{Statistics}

The experiments were run in duplicate and triplicate on different days using the average of the duplicates as one value. The data were analyzed using the GraphPad Prism software ( $\mathrm{V}$ 5.00, GraphPad Software, San Diego, CA, USA), tested for normality using the D'Agostino \& Pearson omnibus normality test, and examined with the non-parametric one-way ANOVA Kruskal-Wallis test followed by Dunn's Multiple Comparison Test or Mann-Whitney U test to compare the two sets of treatment. For comparing with controls set to $100 \%$, the Wilcoxon signed-rank test was used. Significance level was set to $p<0.05$. Significance is indicated as: $*=p<0.05 ; * *=p<0.01 ; * * *=p<0.0013$. 


\section{Results}

\subsection{Analytical Characterization of Glycated Casein}

To assess the effects of AGEs on human macrophage-like cells, AGEs were made in the form of glycated casein, combining casein, lactose, and glucose. In order to monitor the formation of AGEs in the glycated casein, AGE-specific fluorescence was recorded during the heating process. The fluorescence signal increased until 90 min of heating. AGE-specific fluorescence did not further increase between $90 \mathrm{~min}$ to $120 \mathrm{~min}$ of heating (figure 1). CML, CEL, MG-H1 and pentosidine were quantified in glycated casein using UPLC-MS/MS. Pentosidine was undetectable in the samples. The formation of CML, CEL and MG-H1 in glycated casein during the 120-min heating period is shown in figure 2 . The concentrations of all three compounds increased with time of heating. At $15 \mathrm{~min}$ of heating, MG-H1 was present in concentrations of $1.6 \pm 0.9 \mu \mathrm{g} / \mathrm{mL}$ (mean \pm standard $\mathrm{d}$ eviation (SD)), being slightly higher than $0.6 \pm 0.5 \mu \mathrm{g} / \mathrm{ml} \mathrm{CML}$ although not significantly. Between 60 and 120 minutes of heating, $\mathrm{CML}$ concentrations increased from $4.3 \pm 0.8 \mu \mathrm{g} / \mathrm{mL}$ to $11.3 \pm 1.7 \mu \mathrm{g} / \mathrm{mL}$, making CML the most abundant AGE present in glycated casein after $2 \mathrm{~h}$ heating. CEL concentrations increased as well, but were an order of magnitude lower than $\mathrm{CML}$ and reached concentrations of $0.3 \pm 0.2 \mu \mathrm{g} / \mathrm{mL}$ after $120 \mathrm{~min}$ of cooking.

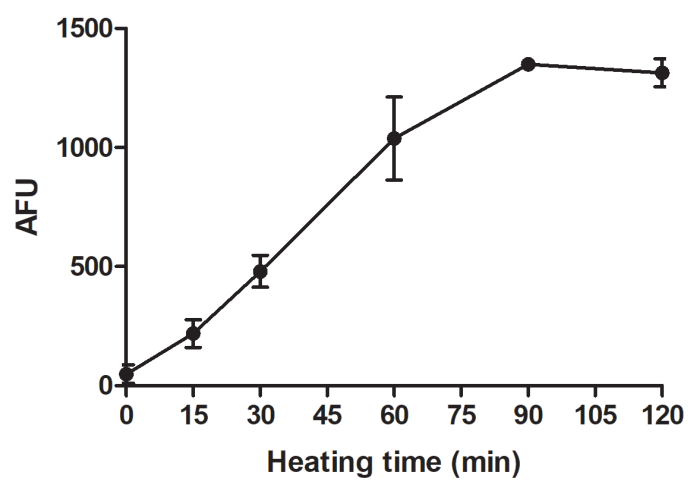

Figure 1. AGE-specific fluorescence of glycated casein measured at $\lambda e x=370 \mathrm{~nm} / \lambda \mathrm{em}=440 \mathrm{~nm}$ in relation to the heating time of the three components casein from bovine milk (10 g/L), lactose $(0.2 \mathrm{M})$ and glucose $(11 \mathrm{mM})$. Values were corrected for baseline fluorescence and data are shown as mean $\pm S D, n=3$. 
A

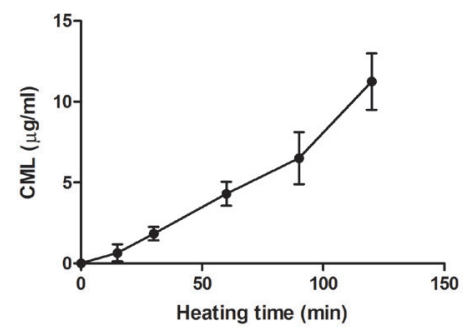

C

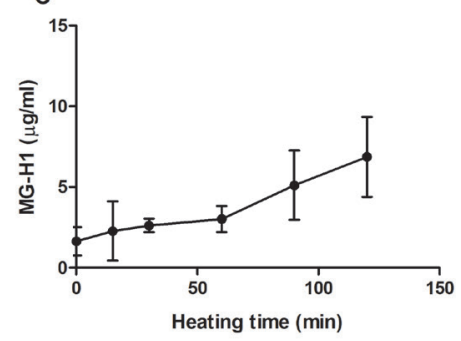

B

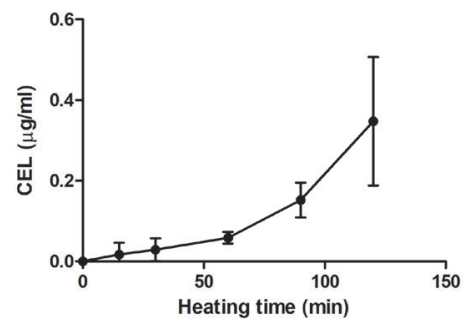

Figure 2. Quantification of $d A G E s$, i.e. concentrations of (A) NE-carboxymethyllysine (CML), (B) carboxyethyllysine (CEL), and (C) methylglyoxal-derived hydroimidazolone (MG-H1), during 120 min heating of a mixture of $10 \mathrm{~g} / \mathrm{L}$ casein, $0.2 \mathrm{M}$ lactose and $11 \mathrm{mM}$ glucose measured by UPLC-MS/MS. Data are presented as mean $\pm \mathrm{SD}, \mathrm{n}=3$.

\subsection{Effects of Glycated Casein on TNF- $\alpha$ Release of Human Macrophage-Like Cells}

In order to assess the inflammatory effects of glycated casein on human macrophage-like cells, THP-1 cells were exposed to glycated casein heated for $15 \mathrm{~min}$. Six-hours exposure of THP-1 cells to various concentrations of glycated casein elevated the release of TNF- $\alpha$ in a concentration-dependent manner (figure 3). Increasing the glycated casein concentration from 1 to $2.5 \%(\mathrm{~V} / \mathrm{V})$ led to an increase of $277 \pm 170 \%$ TNF- $\alpha$ secretion (mean \pm SD), whereas the increase from 5 to $10 \%(\mathrm{v} / \mathrm{v})$ resulted in an increase of $141 \pm 87 \%$. Due to large standard deviations, only the increase of concentration from 1 to $10 \%$ glycated casein led to a significant increase of TNF- $\alpha$ secretion, $1584 \pm 604 \%$ ( $p<0.01)$. In figure 1 and figure 2 , an increase in AGE formation during prolonged heating at $100^{\circ} \mathrm{C}$ is shown. To explore the effects of glycated casein on the viability of macrophage-like cells at different time points during the heating process, the cells were exposed to a $10 \%(v / v)$ solution for $6 \mathrm{~h}$. Cell viability did not alter with increasing cooking time until 120 min of heating, at which the cell viability significantly decreased from $118 \pm 11 \%$ to $86 \pm 21 \%$ (figure 4 ).

To investigate the influence of prolonged heating on the pro-inflammatory effects of glycated casein, we measured the TNF- $\alpha$ release of macrophages exposed for $6 \mathrm{~h}$ to glycated casein $(1 \%(v / v))$, which was heated for $15-120 \mathrm{~min}$. As figure 5 shows, TNF- $\alpha$ secretion did not change significantly between the different heating time intervals. There seems to be an increasing trend, but standard deviations are too large to draw that conclusion. Before adding the glycated casein to the cells, all samples were tested for endotoxin presence; the kit sensitivity was $0.06 \mathrm{EU} / \mathrm{mL}$. No endotoxin was detected above these levels. 


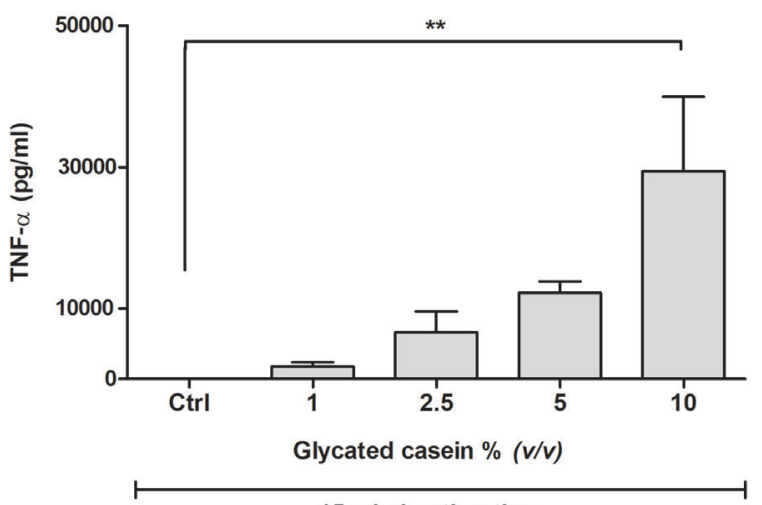

15 min heating time

Figure 3. TNF- $\alpha$ secretion of human macrophage-like cells after $6 \mathrm{~h}$ exposure to different concentrations of glycated casein heated for 15 min measured by ELISA. Data are presented as mean $\pm S D, n=3,{ }^{* *}=p<0.01$.

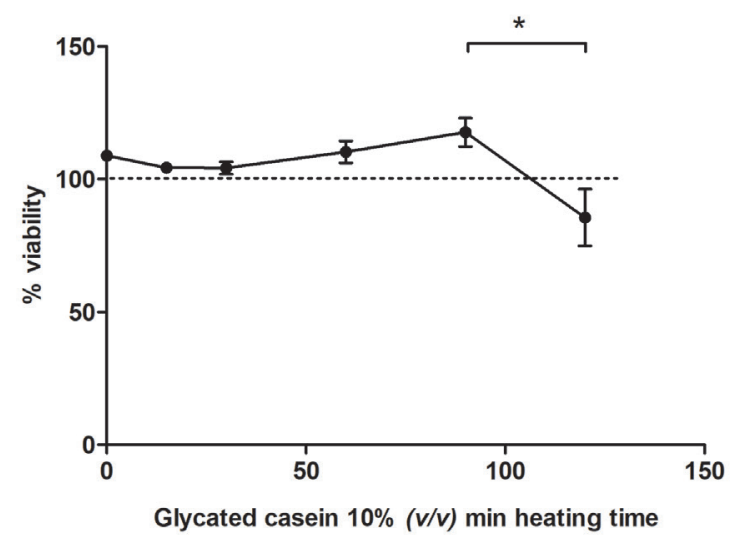

Figure 4. Cell viability of human macrophage-like cells after $6 \mathrm{~h}$ exposure to glycated casein from different heating time points $(10 \% \mathrm{v} / \mathrm{v})$, measured by the MTT assay. Results were normalized to control ( $50 \% \mathrm{v} / \mathrm{v}$ DPBS), which was set to $100 \%$ (dotted line). Data are presented as mean $\pm S D, n=4, *=p<0.05$.

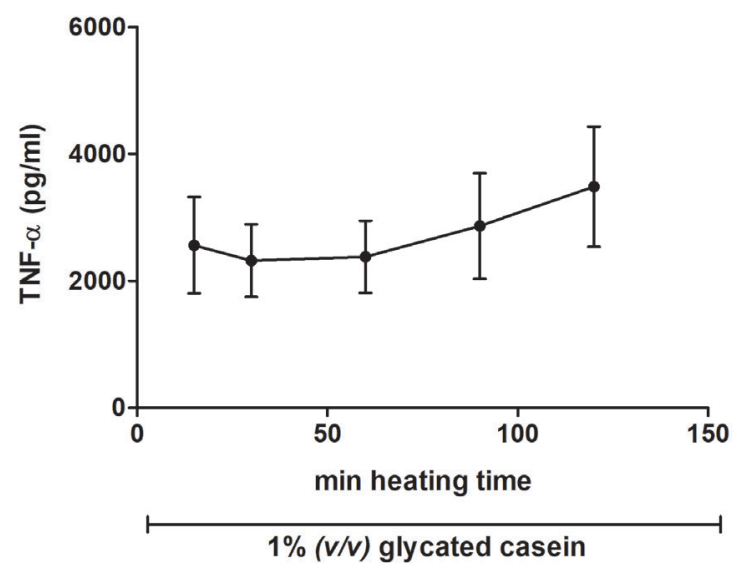

Figure 5. TNF- $\alpha$ secretion of human macrophage-like cells after $6 \mathrm{~h}$ exposure to $1 \%(\mathrm{v} / \mathrm{v})$ glycated casein from different heating time points measured by ELISA. Data are presented as mean $\pm S D, n=4$. 


\subsection{Effects of the AGEs in Glycated Casein on TNF- $\alpha$ Release of Human Macrophage-Like}

\section{Cells}

To confirm that the inflammatory reaction was caused by AGEs, an anti-CML antibody was added to mitigate the TNF- $\alpha$ secretion. Adding the anti-CML antibody to cells exposed to $2.5 \%(v / V)$, glycated casein reduced TNF- $\alpha$ secretion by $36 \pm 24 \%$ ( $p=0.1$, figure 6$)$. Next to this, the cells were incubated with $10 \%(v / v)$ glycated casein with and without $1 \mu \mathrm{M}$ of the RAGE antagonist FPS-ZM1. Addition of $1 \mu \mathrm{M}$ of FPS-ZM1 led to a significant $30 \%$ decrease of TNF- $\alpha$ secretion.

\subsection{Effects of Individual AGEs on TNF- $\alpha$ Release of Human Macrophage-Like Cells}

The cells were incubated with CML, CEL, MG-H1, and acrylamide in different conditions for $6 \mathrm{~h}$ to assess the effect of individual AGEs on TNF- $\alpha$ release. All compounds were tested in concentrations ranging from $0.1 \mathrm{mM}$ to $3 \mathrm{mM}$. None of the individual compounds led to any TNF- $\alpha$ secretion by the macrophage-like cells (data not shown).

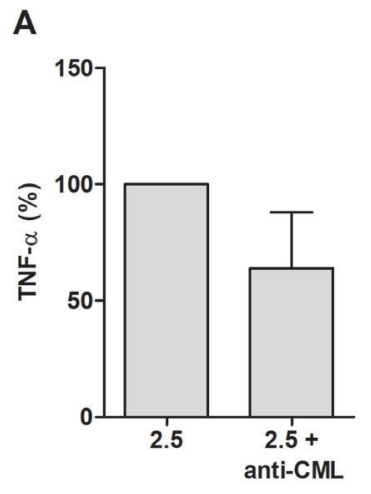

Glycated casein \% (v/v)
B

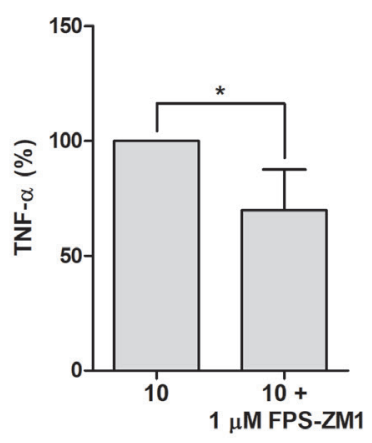

Glycated casein \% (v/v)

Figure 6. (A) TNF- $\alpha$ secretion of human macrophage-like cells after $6 \mathrm{~h}$ exposure to $2.5 \%(\mathrm{v} / \mathrm{v})$ glycated casein with or without anti-CML antibody measured by ELISA. After addition of an anti-CML antibody, TNF- $\alpha$ secretion decreased by $36 \% \pm 24 \%$. Data are presented as mean $\pm S D, n=3$. (B) TNF- a secretion of human macrophage-like cells after $6 \mathrm{~h}$ exposure to $10 \%(\mathrm{v} / \mathrm{V})$ glycated casein with or without $1 \mu \mathrm{M}$ RAGE antagonist (FPS-ZM1) measured by ELISA. After addition of the RAGE blocker, TNF- $\alpha$ secretion decreased by $30 \pm 18 \%$. Data are presented as mean \pm SD, $n=4, *=p<0.05$. 


\section{Discussion}

In this study, we showed that dietary AGEs directly led to a concentration-dependent increase in TNF- $\alpha$ secretion from human macrophage-like cells, which is induced through the binding to RAGE. Our AGEs were made in a home cooking environment at $100^{\circ} \mathrm{C}$ and are therefore much more representative for dietary AGEs formed during food processing and cooking than AGEs formed at $37^{\circ} \mathrm{C}$. The presence of AGEs in the glycated casein was confirmed by UPLC-MS/MS, and testing the glycated casein for endotoxin presence eliminated the possibility of the TNF- $\alpha$ secretion to be induced by endotoxins. Exposing macrophage-like cells to the glycated casein induced a clear TNF- $\alpha$ secretion. This opposes the findings of Buetler et al., where eliminating endotoxins by TX-114 extraction diminished the capability of their solutions to induce TNF- $\alpha$ secretion in human cell lines [13]. Further differences between our study and that of Buetler et al., and others, is firstly their use of endogenous AGEs developed at $38^{\circ} \mathrm{C}$ to our dietary AGEs, which are developed at $100^{\circ} \mathrm{C}$ and secondly their use of human lung epithelial cells and human retinal pigment epithelial cell line opposed to our human immune cells. Immune cells are naturally much more prone to excrete cytokines. Interestingly, Cai et al. fractioned AGEs from food products and showed their involvement in intracellular oxidative stress and some inflammatory potential in human umbilical vein endothelial cells. This underlines our results that dietary AGEs induce cellular stress [16].

The glycated casein induced TNF- $\alpha$ secretion could be attenuated by anti-CML antibodies, showing that the AGEs directly induce TNF- $\alpha$ secretion. Addition of the anti-CML antibody did, however, not completely diminish the TNF- $\alpha$ secretion. Since CML is not the only AGE present in the glycated casein, this finding indicates that other AGEs can also exert an inflammatory effect. Our observation that only adding CML antibodies can mitigate the inflammatory effect induced by glycated casein is quite remarkable. Blocking RAGE with the antagonist FPS-ZM1 showed that the effect was caused by AGEs binding to RAGE. These combined results corroborate our conclusion that the TNF- $\alpha$ secretion is induced by AGEs specifically. The most well-known pathway for TNF- $\alpha$ production through RAGE stimulation is NF-KB activation via activation of mitogen-activated protein (MAP) kinases, leading to TNF- $\alpha$ secretion [17]. Indeed, this has been shown by Lander et al. in rat pulmonary smooth muscle cells. It would be of interest to investigate this in human macrophages in future experiments. Opposed to our results from glycated casein, the individual AGES CML, CEL, MG-H1 and acrylamide did not show any induction of TNF- $\alpha$ release in human macrophage-like cells. This result indicates that AGEs need to be bound to proteins to exert an inflammatory reaction. This hypothesis has been mentioned in multiple studies [18,19]. Remarkably, a lot of conflicting research exists on whether individual AGEs can bind to RAGE and thereby induce an inflammatory response and whether they need to be bound to proteins to have this effect. Our results underline the hypothesis that AGEs can exert an inflammatory reaction through binding to RAGE, but only when bound to proteins. The reason why individual AGEs do not bind to RAGE has to be investigated further.

What would the results of this study imply for the human situation? Endogenously generated 
AGEs are known to induce significant adverse health effects when formed in the human body at $37^{\circ} \mathrm{C}$. AGEs can bind to RAGE, thereby activating an intracellular signaling cascade ending in the excretion of pro-inflammatory cytokines, leading to metabolic diseases such as atherosclerosis $[10,20,21]$. Another important mechanism by which AGEs can cause damage to the human body is by crosslinking with endogenous proteins such as collagen and elastin and thereby increasing stiffness in, for instance, muscle tissue [22]. In this study, we showed that dietary AGEs can also induce pro-inflammatory cytokine secretion. If AGEs in food are able to enter the human body, they might be able to contribute to the body's endogenous AGE pool and worsen specific metabolic diseases. Food products that contain AGEs are those high in proteins and sugars and are processed at high temperatures, such as baked meats, cereals, peanut butter, and chocolate $[6,7]$. It is currently discussed whether dietary AGEs are absorbed in the gastrointestinal tract and contribute to the endogenous AGE pool [23-26]. Upon absorption, these dietary AGE products may aggravate metabolic diseases by the production of pro-inflammatory cytokines, as our study shows. Independently from this question of absorption, it should be emphasized that the pro-inflammatory effects of AGE may also be of local relevance in the gastrointestinal tract and might contribute to the symptoms of diseases such as inflammatory bowel diseases, Crohn's disease, and ulcerative colitis.

\section{Conclusions}

In conclusion, our study revealed that dietary AGEs themselves stimulate TNF- $\alpha$ secretion in human macrophage-like cells. To what extent dietary AGEs pose a risk for developing inflammatory diseases in the human GI tract remains to be investigated.

\section{Acknowledgments}

The authors would like to thank Stefan van Leeuwen for his valuable help at RIKILT (now WFSR) and Jacques Davies for performing the LC-MS/MS measurements. 


\section{References}

1. Nguyen, H.T.; van der Fels-Klerx, H.J.; van Boekel, M.A. Kinetics of N(epsilon)-(carboxymethyl Jysine formation in aqueous model systems of sugars and casein. Food Chemi. 2016, 192, 125-133, doi:10.1016/j.foodchem.2015.06.110.

2. Pratt, C.W.; Cornely, K. Essential Biochemistry, 3rd ed.; John Wiley distributor: Hoboken, N.J., Chichester, USA, 2014; pp. 626; ISBN 13 978-1118-08350-5.

3. Poulsen, M.W.; Hedegaard, R.V.; Andersen, J.M.; de Courten, B.; Bugel, S.; Nielsen, J.; Skibsted, L.H.; Dragsted, L.O. Advanced glycation endproducts in food and their effects on health. Food Chem. Toxicol. 2013, 60, 10-37, doi:10.1016/j.fct.2013.06.052.

4. Goldberg, T.; Cai, W.; Peppa, M.; Dardaine, V.; Baliga, B.S.; Uribarri, J.; Vlassara, H. Advanced glycoxidation end products in commonly consumed foods. J. Am. Diet. Assoc. 2004, 104, 1287-1291, doi:10.1016/j.jada.2004.05.214.

5. Uribarri, J.; Woodruff, S.; Goodman, S.; Cai, W.; Chen, X.U.E.; Pyzik, R.; Yong, A.; Striker, G.E.; Vlassara, $\mathrm{H}$. Advanced glycation end products in foods and a practical guide to their reduction in the diet. J. Am. Diet. Assoc. 2010, 110, 911-916, doi:10.1016/j. jada.2010.03.018.

6. Scheijen, J.L.; Clevers, E.; Engelen, L.; Dagnelie, P.C.; Brouns, F.; Stehouwer, C.D.; Schalkwijk, C.G. Analysis of advanced glycation endproducts in selected food items by ultraperformance liquid chromatography tandem mass spectrometry: Presentation of a dietary AGE database. Food Chem. 2016, 190, 1145-1150, doi:10.1016/j. foodchem.2015.06.049.

7. Hull, G.L.J.; Woodside, J.V.; Ames, J.M.; Cuskelly, G.J. N-epsilon-(carboxymethyl)lysine content of foods commonly consumed in a Western style diet. Food chem. 2012, 131, 170-174, doi:10.1016/j.foodchem.2011.08.055.

8. Ahmed, N.; Mirshekar-Syahkal, B.; Kennish, L.; Karachalias, N.; Babaei-Jadidi, R.; Thornalley, P.J. Assay of advanced glycation endproducts in selected beverages and food by liquid chromatography with tandem mass spectrometric detection. Mol. Nutr. Food Res. 2005, 49, 691-699, doi:10.1002/mnfr.200500008.

9. Frati, G.; Schirone, L.; Chimenti, I.; Yee, D.; Biondi-Zoccai, G.; Volpe, M.; Sciarretta, S. An overview of the inflammatory signalling mechanisms in the myocardium underlying the development of diabetic cardiomyopathy. Cardiovasc. Res. 2017, 113, 378-388, doi:10.1093/cvr/cvx011.

10. Xie, J.; Mendez, J.D.; Mendez-Valenzuela, V.; Aguilar-Hernandez, M.M. Cellular signalling of the receptor for advanced glycation end products (RAGE). Cell. Signal. 2013, 25, 21852197, doi:10.1016/j.cellsig.2013.06.013.

11. Friedman, M. Chemistry, biochemistry, and safety of acrylamide. A review. J. Agric. Food Chem. 2003, 51, 4504-4526, doi:10.1021/jf030204+.

12. Kumar, J.; Das, S.; Teoh, S.L. Dietary acrylamide and the risks of developing cancer: Facts to ponder. Front Nutr. 2018, 5, 14, doi:10.3389/fnut.2018.00014.

13. Buetler, T.M.; Latado, H.; Leclerc, E.; Weigle, B.; Baumeyer, A.; Heizmann, C.W.; Scholz, G. Glycolaldehyde-modified beta-lactoglobulin AGEs are unable to stimulate inflammatory signaling pathways in RAGE-expressing human cell lines. Mol. Nutr. Food Res. 2011, 55, 291-299, doi:10.1002/mnfr.201000140.

14. Klenovics, K.S.; Boor, P.; Somoza, V.; Celec, P.; Fogliano, V.; Sebekova, K. Advanced glycation end products in infant formulas do not contribute to insulin resistance associated with their consumption. PloS one 2013, 8, e53056, doi:10.1371/journal.pone.0053056.

15. Assar, S.H.; Moloney, C.; Lima, M.; Magee, R.; Ames, J.M. Determination of Nepsilon(carboxymethyl)lysine in food systems by ultra performance liquid chromatography-mass spectrometry. Amino Acids 2009, 36, 317-326, doi:10.1007/s00726-008-0071-4.

16. Cai, W.; Gao, Q.D.; Zhu, L.; Peppa, M.; He, C.; Vlassara, H. Oxidative stress-inducing carbonyl compounds from common foods: novel mediators of cellular dysfunction. Mol. Med. 2002, 8, 337-346.

17. Lander, H.M.; Tauras, J.M.; Ogiste, J.S.; Hori, O.; Moss, R.A.; Schmidt, A.M. Activation of the receptor for advanced glycation end products triggers a p21(ras)-dependent mitogenactivated protein kinase pathway regulated by oxidant stress. J. Bio. Chem. 1997, 
272, 17810-17814.

18. Kislinger, T.; Fu, C.; Huber, B.; Qu, W.; Taguchi, A.; Du Yan, S.; Hofmann, M.; Yan, S.F.; Pischetsrieder, M.; Stern, D., et al. N(epsilon)-(carboxymethyl)lysine adducts of proteins are ligands for receptor for advanced glycation end products that activate cell signaling pathways and modulate gene expression. J. Bio. Chem. 1999, 274, 31740-31749.

19. Buetler, T.M.; Leclerc, E.; Baumeyer, A.; Latado, H.; Newell, J.; Adolfsson, O.; Parisod, V.; Richoz, J.; Maurer, S.; Foata, F., et al. N-epsilon-carboxymethyllysine-modified proteins are unable to bind to RAGE and activate an inflammatory response. Mol. Nutr. Food Res. 2008, 52, 370-378, doi:DOI 10.1002/mnfr.200700101.

20. Kierdorf, K.; Fritz, G. RAGE regulation and signaling in inflammation and beyond. J. Leukoc. Biol. 2013, 94, 55-68, doi:10.1189/jlb.1012519.

21. Angoorani, P.; Ejtahed, H.S.; Mirmiran, P.; Mirzaei, S.; Azizi, F. Dietary consumption of advanced glycation end products and risk of metabolic syndrome. Int. J. Food Sci. Nutr. 2016, 67, 170-176, doi:10.3109/09637486.2015.1137889.

22. Van Puyvelde, K.; Mets, T.; Njemini, R.; Beyer, I.; Bautmans, I. Effect of advanced glycation end product intake on inflammation and aging: A systematic review. Nutr. Rev. 2014, 72, 638-650, doi:10.1111/nure.12141.

23. Geissler, S.; Hellwig, M.; Zwarg, M.; Markwardt, F.; Henle, T.; Brandsch, M. Transport of the advanced glycation end products alanylpyrraline and pyrralylalanine by the human proton-coupled peptide transporter hPEPT1. J. Agric. Food Chem. 2010, 58, 2543-2547, doi:10.1021/jf903791u.

24. Hellwig, M.; Geissler, S.; Peto, A.; Knutter, I.; Brandsch, M.; Henle, T. Transport of free and peptide-bound pyrraline at intestinal and renal epithelial cells. J. Agric. Food Chem. 2009, 57, 6474-6480, doi:10.1021/jf901224p.

25. Hellwig, M.; Matthes, R.; Peto, A.; Lobner, J.; Henle, T. N-epsilon-fructosyllysine and $\mathrm{N}$-epsilon-carboxymethyllysine, but not lysinoalanine, are available for absorption after simulated gastrointestinal digestion. Amino Acids 2014, 46, 289-299, doi:10.1007/ s00726-013-1501-5.

26. Alamir, I.; Niquet-Leridon, C.; Jacolot, P.; Rodriguez, C.; Orosco, M.; Anton, P.M.; Tessier, F.J. Digestibility of extruded proteins and metabolic transit of $\mathrm{N}$-epsilon-carboxymethyllysine in rats. Amino Acids 2013, 44, 1441-1449, doi:10.1007/s00726-012-1427-3. 


\section{Supplements}

Table S1. Target compounds and selected instrumental parameters for quantification of each compound by UPLC$($ ESI+)MS/MS.

\begin{tabular}{|c|c|c|c|c|}
\hline Compound & $\begin{array}{l}\text { Precursor }> \\
\text { product ion }(\mathrm{m} / \mathrm{z})\end{array}$ & Cone voltage (V) & $\begin{array}{l}\text { Collision energy } \\
(\mathrm{eV})\end{array}$ & $\begin{array}{l}\text { Internal } \\
\text { Standard used }\end{array}$ \\
\hline \multirow{2}{*}{ CML } & $205.2>84.2$ & 20 & 20 & \multirow{2}{*}{$C M L-d 2$} \\
\hline & $205.2>130.2$ & 20 & 12 & \\
\hline \multirow{2}{*}{ CEL } & $219.2>84.2$ & 20 & 20 & \multirow{2}{*}{ CEL-d4 } \\
\hline & $219.2>130.2$ & 20 & 10 & \\
\hline \multirow{2}{*}{ MG-H1 } & $229.2>114.1$ & 20 & 13 & \multirow{2}{*}{ MG-H1-d3 } \\
\hline & $229.2>166.2$ & 20 & 13 & \\
\hline \multirow{2}{*}{$\begin{array}{l}\text { Pentosi- } \\
\text { dine }\end{array}$} & $379.4>135.1$ & 20 & 40 & \multirow{2}{*}{ MG-H1-d3 } \\
\hline & $379.4>187.1$ & 20 & 35 & \\
\hline
\end{tabular}

Note: the underlined precursor $>$ product ion transition was used for quantification, the italic precursor $>$ product ion transition was used for confirmation.

Table S2. Instrumental detection and quantitation limits (pg on column) and method detection and quantitation limits (pg on column)

\begin{tabular}{lllll}
\hline Compound & $\begin{array}{l}\text { IDL } \\
\text { (pg on column) }\end{array}$ & $\begin{array}{l}\text { IQL } \\
\text { (pg on column) }\end{array}$ & $\begin{array}{l}\text { MDL } \\
\text { ( } \boldsymbol{\mu g} / \mathbf{m l})\end{array}$ & $\begin{array}{l}\text { MQL } \\
\text { ( } \boldsymbol{\mu g} / \mathbf{m l})\end{array}$ \\
\hline $\mathrm{CML}$ & 0.5 & 1.5 & 0.001 & 0.003 \\
\hline $\mathrm{CEL}$ & 1 & 3.5 & 0.01 & 0.03 \\
\hline MG-H1 & 1.7 & 5.7 & 0.03 & 0.1 \\
\hline Pentosidine & 6.4 & 21 & 1.2 & 4 \\
\hline
\end{tabular}



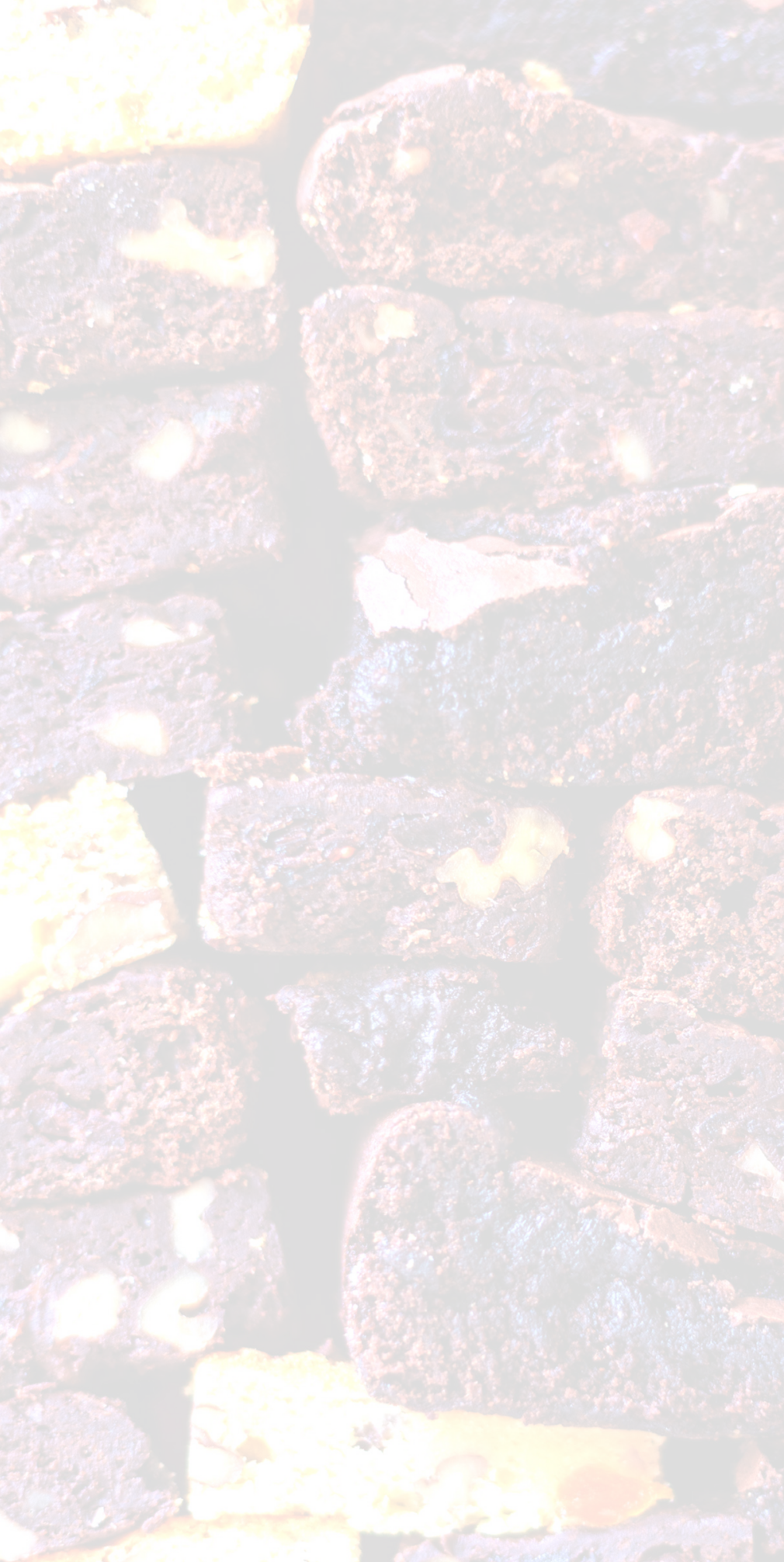

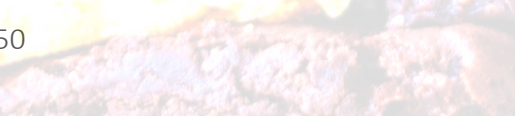




\section{Chapter 3}

\section{Gastrointestinal digestion of dietary advanced glycation endproducts using an in vitro model of the gastrointestinal tract (TIM-1)}

Timme van der Lugt, Koen Venema, Stefan van Leeuwen, Misha F. Vrolijk, Antoon Opperhuizen, Aalt Bast

Food \& Function, 2020 Jul 22; 11(7): 6297-6307 


\section{Abstract}

Protein- and sugar-rich food products processed at high temperatures contain large amounts of dietary advanced glycation endproducts (dAGEs). Our earlier studies have shown that specifically protein-bound dAGEs induce a pro-inflammatory reaction in human macrophagelike cells. To what extent these protein-bound dAGEs survive the human gastrointestinal (GI) tract is still unclear. In this study we analysed gastric and small intestinal digestion of dAGEs using the validated, standardised TNO in vitro gastrolntestinal digestion Model (TIM-1), a dynamic in vitro model which mimics the upper human GI tract. This model takes multiple parameters into account, such as: dynamic pH curves, peristaltic mixing, addition of bile and pancreatic digestive enzymes, and passive absorption. Samples of different digested food products were collected at different time points after (i) only gastric digestion and (ii) after both gastric plus small intestinal digestion. Samples were analysed for dAGEs using UPLC-MS/ MS for the lysine derived $\mathrm{N} \varepsilon$-carboxymethyllysine (CML) and $\mathrm{N} \varepsilon$-carboxyethyllysine (CEL), and the arginine derived methylglyoxal-derived hydroimidazolone-1 (MG-H1), and glyoxalderived hydroimidazolone-1 (G-H1). All AGEs were quantified in their protein-bound and free form. The results of this in vitro study show that protein-bound dAGEs survive gastrointestinal digestion and are additionally formed during small intestinal digestion. In ginger biscuits, the presence MG-H1 in the $\mathrm{Gl}$ tract increased with more than $400 \%$. This also indicates that dAGEs enter the human GI tract with potential pro-inflammatory characteristics. 


\section{Introduction}

A typical characteristic of the Western diet is the abundance of fried and otherwise processed and heat-treated foods. During thermal processing of food, large amounts of dietary advanced glycation endproducts (dAGEs) are formed [1]. Especially in food products containing ample amounts of carbohydrates and proteins, dAGEs are highly present. This formation of dAGEs occurs via the Maillard reaction (figure 1 ) in which the carbonyl group of a reducing sugar reacts with an amino moiety of an amino acid under the influence of heat [2]. dAGEs are formed at the final stage of this reaction and are largely responsible for the brown colour and palatability of heat-processed food. Although in some studies dAGES are considered to play an important role in the negative health effects attributed to the Western diet, the health consequences of these compounds are not yet well understood. In a recent study, we demonstrated pro-inflammatory effects of dAGEs on human macrophagelike cells through binding to the receptor for advanced glycation endproducts (RAGE) [3]. Only protein-bound dAGEs were found to have this pro-inflammatory effect, which was measured as TNF- $\alpha$ secretion, whereas free $d A G E s$, such as $N \varepsilon$-carboxymethyllysine $(\mathrm{CML})$ and methylglyoxalderived-hydroimidazolone-1 (MG-H1), did not induce any TNF- $\alpha$ secretion.

Since the dAGE-protein binding may be vulnerable to acid hydrolysis and proteolysis that occur during gastrointestinal (GI) digestion, it is to be expected that Gl digestion may influence the pro-inflammatory effects. After the ingestion of protein-rich food products, several mechanical and enzymatic processes break down the ingested proteins during GI digestion. In the stomach, acid hydrolysis occurs and the proteolytic enzyme pepsin cleaves proteins into smaller polypeptides. In the small intestine the $\mathrm{pH}$ is increased to a neutral level and different proteolytic enzymes from the pancreatic juice cleave the polypeptides further to dipeptides and peptides, which become then available for absorption. The possible release of the AGEs from proteins into their free form may result in a reduced pro-inflammatory effect of dAGEs. Thus far, the influence of GI digestion on the pro-inflammatory potency of dAGEs has not been investigated.

The existing evidence on the effects of digestion in dAGEs is conflicting. While some studies found breakdown of protein binding during digestion [4, 5], most studies found a survival of protein bound AGEs in the GI tract [6-8]. Moreover, it has previously been suggested that especially high molecular weight dAGES, which include the protein-bound dAGEs, were able to inhibit intestinal protease activity. Already in 1993, Pitotti et al. found an inhibiting effect of Maillard reaction products on protease activity to glycated proteins [9]. However, one of the issues that arise when assessing these studies for human relevance, is the use of static GI digestion in vitro models. Often, the stomach phase of these models consists of a mixture of digestion enzymes with a constant $\mathrm{pH}$ of $2-2.5$ where the food products are exposed to for two hours. After the stomach phase the digests are transferred to a new static environment consisting of different buffers and digestion enzymes with a constant $\mathrm{pH}$ of $6.5-7.5$ for $4-$ 
6 hours [6-8]. In contrast to these static GI digestion in vitro models, the human Gl tract is a dynamic system that includes mixing via peristaltic movement and alternating $\mathrm{pH}$ levels. Additionally, digestive fluids and meal components are removed from the Gl tract by both passive and active absorption [10]. The TNO gastrolntestinal Model (TIM-1) is a dynamic in vitro model that is able to mimic much of the human Gl tract. This model takes multiple parameters into account, such as: dynamic pH curves, peristaltic mixing, addition of bile and pancreatic enzymes, and passive absorption [11]. The TIM-1 model accurately mimics the in vivo food and drug behaviour with a high prediction capability compared to other models [12]. The studies that have been performed on the effect of GI digestion on dAGEs thus far, not only poorly reflect the in vivo situation, they also did not use accurate methods to measure dAGEs. These methods include fluorescence and/or competitive ELISA of CML and 'methylglyoxal- derived AGEs' [13]. Additionally, most studies only assessed meal-resembling systems, and most of these studies focussed on the effect of glycation on protein digestion instead of the digestion of the dAGEs themselves.

Therefore, in this study we aimed to investigate to what extent the dAGE-protein binding is affected by human GI digestion. This is done by digesting a food-based matrix and actual food products (ginger biscuits and apple juice) using the TIM-1 GI model and analysing the digests for different free-dAGEs and protein-bound dAGEs by ultrahigh pressure liquid chromatography coupled to triple quadrupole mass spectrometry (UPLC-MS/MS).
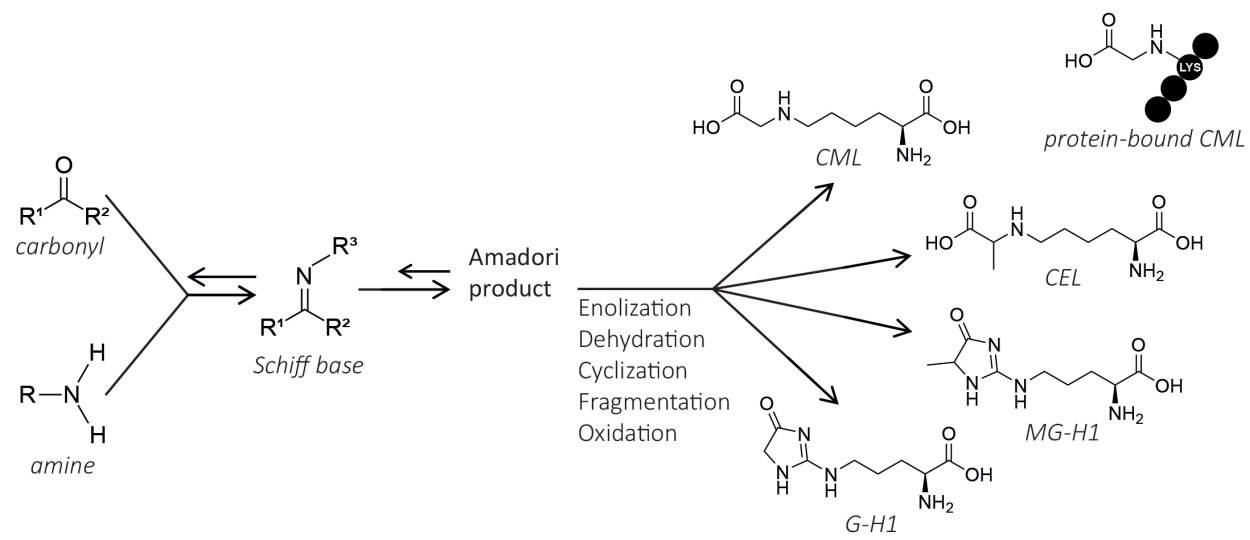

Figure 1. Simplified overview on AGE formation via the Maillard reaction. As an example, the molecular structure of four different dAGEs: NE-carboxymethyllysine (CML), NE-carboxyethyllysine (CEL), glyoxalderived-hydroimidazolone-1 (G-H1), and methylglyoxalderived-hydroimidazolone-1 (MG-H1), as well as protein-bound CML are shown. 


\section{Materials and methods}

\subsection{Chemicals and reagents}

Casein from bovine milk, $\alpha$-lactose monohydrate, $\mathrm{NaOH}$, sodium-phosphate, and 2-mercaptoethanol were obtained from Sigma-Aldrich (Saint Louis, MO USA). Pancreatin (Pancrex V) was obtained from Paines and Birne (Greenford, UK). Porcine bile extract was obtained from Merck (Darmstadt, Germany). Analytical standards of CML (>99\%), CEL (>98\%), GH-1 (98.2\%) and MG-H1 (>98.2\%), as well as the deuterium labelled internal standards CML-D4, CEL-D4, and MG-H1-D3 and G-H1-13C2, were obtained from Iris Biotech (Marktredwitz, Germany). Boric acid (99.5\%), chloroform (99.5\%), nonafluoropentanoic acid (NFPA; 99\%), sodium hydroxide (98\%), sodium borohydride (96\%), and trifluoroacetic acid (TFA; 99\%), were obtained from Sigma (Zwijndrecht, Netherlands). HPLC-grade acetonitrile and methanol were obtained from Actu-all Chemicals (Oss, Netherlands).

\subsection{Preparation of samples}

Glycated casein (GC) for use as food-based matrix was prepared by combining casein, glucose, and lactose, in the double proportions of milk powder (22 mM glucose, $0.4 \mathrm{M}$ lactose, $20 \mathrm{~g} / \mathrm{L}$ casein from bovine milk), and diluted in $50 \mathrm{mM}$ phosphate buffer, $\mathrm{pH}$ 7.4. One part of the mixture was aliquoted and stored at $-80^{\circ} \mathrm{C}$ for use as control sample (unglycated casein (UC)). The remaining mixture was heated in an Erlenmeyer on a heating plate at $100^{\circ} \mathrm{C}$ for 1 . After 1 samples were taken and immediately cooled in ice water. Samples were aliquoted and stored at $-80^{\circ} \mathrm{C}$. The food products ginger biscuits and apple juice from the same respective batches were bought at a local Dutch supermarket.

\subsection{TNO in vitro model of the stomach and small intestine (TIM-1)}

Figure S1 shows a schematic of the in vitro model, which has been described extensively before [14]. Briefly, the model comprises four connected glass compartments, representing the stomach, duodenum, jejunum and ileum, respectively. Inside each glass compartment is a flexible silicone inner wall. The space between the inner and outer walls is filled with water of $37^{\circ} \mathrm{C}$. By periodically applying pressure on the water, the flexible inner walls are squeezed, simulating peristalsis. In each individual compartment the $\mathrm{pH}$ is measured continuously and regulated by 'secretion' of hydrochloric acid (gastric compartment) or sodium bicarbonate (intestinal compartments). The set-points of $\mathrm{pH}$, gastric emptying and intestinal transit time are controlled by a computer. The current experiments were performed in duplicate under the average physiological conditions as found in the human gastrointestinal tract for adults (figure S2). The gastric emptying, intestinal residence time and gastric and intestinal $\mathrm{pH}$-curves mimicked the situation as found in humans for intake after a meal (figure S2). Additional to figure S2, intestinal pH were set at 6.8 in the duodenum, 6.8 in the jejunum, and 7.2 in the ileum. The concentrations of secretion fluids (electrolytes, enzymes, bile, and pancreatic juice) were adjusted to the average concentrations as described for adults after ingestion of a meal. Pancreatic output was simulated by secreting $10 \%$ pancreatin (protease, 
lipase, and amylase) in small intestinal electrolyte solution. Biliary output was simulated by secreting a $2 \%$ bile (porcine bile extract) solution at $0.5 \mathrm{ml} / \mathrm{min}$. Prior to the experiment the compartments were filled with start residues as described before [15], except for the gastric residue, which was mixed with the 'meal'. Hollow fibre membrane systems continuously dialyzed the digested and dissolved low-molecular weight compounds from the jejunum (figure S1-MN left) and ileum compartments (figure S1-MN right), which simulated absorption of nutrients by the body, and which maintained physiological concentrations of bile and electrolytes. The enzyme activity of pepsin was 2500 units/mg. These units are defined as a change in the absorbance at $\lambda=280 \mathrm{~nm}$ of 0.001 per minute at $\mathrm{pH} 2.0$ at $37^{\circ} \mathrm{C}$, measured as trichloroacetic acid (TCA)-soluble products using haemoglobin as substrate. The enzyme activities of the pancreatin was expressed in international units and were: amylase 12000 $\mathrm{IU} / \mathrm{g}$, protease $1000 \mathrm{IU} / \mathrm{g}$, and lipase $15000 \mathrm{IU} / \mathrm{g}$. Experiments were done both in the gastric compartment only, as well as in the complete model. In the experiments in which only the gastric compartment was used, the duodenal compartment was only used for neutralization of the gastric efflux, without secretion of bile and pancreatin. This neutralization occurred before sampling. In the gastric experiments, during 3 hours approximately $95 \%$ of the gastric contents were gradually delivered into the small intestine for neutralization (figure S2) through the 'pyloric sphincter' (figure S1-B). Experiments in the complete model lasted 6 hours, after which approximately $90 \%$ of the small-intestinal contents were gradually delivered (figure S2) into the 'large intestine' (sampling bottle) through the 'ileo-caecal sphincter' (figure S1-H). Samples were taken every hour from the jejunal and ileal diaysates (Figure S1-MN, left and right respectively), and the ileal efflux (figure S1-H). After termination of the experiments, the residues remaining in the system were collected as well, to allow a mass-balance to be made. In case of the liquid food products GC and applejuice, the starting product contained 300 grams of food and for the solid ginger biscuits 30 grams of biscuits were run through the model. All results that were later obtained with the UPLC MS/MS were corrected for the amount of AGEs present in the gastro-intestinal fluids and enzymes of the TIM-1 model by running a control experiment with only $50 \mathrm{mM}$ phosphate buffer.

\subsection{Quantification of dAGEs in digests by ultra-performance liquid chromatography- tandem mass spectrometry (UPLC-MS/MS)}

In this study, every sample was analysed in both the hydrolysed (total dAGEs, i.e. free + protein-bound dAGEs) and unhydrolysed form (free dAGEs). The sample preparation is based on earlier studies $[1,16]$ and similar to our previous study [3]. The technical procedure is described in van der Lugt et al. (2018) [3]. The details on the sample preparation and UPLCMS/MS settings are mentioned in the supplements. The free dAGE content in the samples was analysed by omitting the hydrolysis step in the sample preparation procedure, thereby circumventing the release of protein-bound dAGEs. Quantification was performed using the precursor-product ion multiple reaction monitoring (MRM) transitions reported in table S1. The accuracy of the analysis was monitored by spiking each sample with a specific dAGE 
standard for each analysed dAGE. The average accuracies ranged from 75-133\% (see table S2), demonstrating that no severe losses occurred during sample preparation, and no signal enhancements or suppression occurred during UPLC-MS/MS analysis. Ion ratios between the quantifier ion and qualifier ion were monitored. In case ion ratios deviated more than $20 \%$ from the ratio observed in the standard, the identity of the peak could not be confirmed. In the case of MG-H1 and G-H1, ion-ratio deviations were observed in GC, ginger cookies and apple juice. A brief investigation into this issue revealed that isomers of MG-H1 (i.e. MG-H2 and MG-H3) and G-H1 (i.e. G-H2 and G-H3) may co-exist in the samples. Additional explorative experiments showed that these may co-elute and alter the ion-ratios (data not shown). Future work is needed to explore this work further, but with the isomers issue in consideration, deviating ion ratios were accepted for MG-H1 and $\mathrm{G}-\mathrm{H} 1$. It is expected that this also played a role in the slightly elevated (or lower) accuracies for MG-H1 and G-H1 (table S2). The LOQ for the protein-bound dAGEs was $125 \mu \mathrm{g} / \mathrm{L}$ and $10 \mu \mathrm{g} / \mathrm{L}$ for the free dAGEs (table S3). This 12.5 fold difference can be explained by omitting the acid hydrolysis step, which introduces a dilution of the sample. By omitting this step, the sample is not diluted, resulting in lower LOQs.

\subsection{Analysis of protein size by SDS-page and Coomassie Blue Staining}

A sample of $10 \mu \mathrm{g}$ of digested and undigested GC and UG with reducing laemmli buffer was loaded onto an Any kD ${ }^{\mathrm{TM}}$ Mini-PROTEAN ${ }^{\circledR}$ TGX ${ }^{\mathrm{TM}}$ Precast Protein gel (Bio-rad, Hercules, CA, USA) and run in a Bio-rad cell. Due to volume constraints, the protein amount for small intestinal digested UG was adjusted to $7 \mu \mathrm{g}$. For assessment of protein size, the gels were stained by Coomassie brilliant blue R250 (Bio-Rad) and imaged with Amersham Imager 600 (GE Healthcare, Chicago, IL, USA). For casein assessment, the samples were treated as previously mentioned. After running, the protein was transferred to a Nitrocellulose membrane (BioRad). Primary antibodies used: Anti-Casein (ab166596, Abcam, Cambridge, UK) Secondary antibody used: Anti-rabbit IgG, HRP-linked Antibody (Cell Signaling Technology, Danvers, MA, USA). The membrane was then incubated with Clarity Western ECL Substrate (Bio-rad) for colour development. Staining of the membranes was analysed using Amersham Imager 600.

\subsection{Data assessment}

Every sample was analysed in both the hydrolysed (total dAGEs) and unhydrolysed form (free dAGEs), as discussed above. To obtain the concentration of protein-bound dAGEs in the samples, the results from the free dAGE measurement were subtracted from the total dAGE measurement of each sample and each dAGE. To correct for any dAGEs present in the TIM-1 solutions and enzymes, a control run of TIM-1 with $50 \mathrm{mM}$ phosphate buffer was conducted and analysed using the UPLC-MS/MS. The small quantities found in the digests from phosphate buffer were subtracted from all dAGE results in the samples. The UPLC$\mathrm{MS} / \mathrm{MS}$ results (in $\mu \mathrm{g} / \mathrm{ml}$ ) were then multiplied by the volume of the effluents to obtain the absolute amount of dAGEs. 


\section{Results}

\subsection{Digestion of dietary AGEs - protein binding}

\subsubsection{Glycated casein (GC)}

The digestion of protein-bound dAGE was assessed with the TIM-1 using a food-based matrix containing a mixture of heated glucose, lactose and casein. After running the samples through the model, the digests were analysed for the presence of protein-bound and free dAGES using UPLC-MS/MS.

The starting product (undigested $\mathrm{GC}, \mathrm{t}=0 \mathrm{~h}$ ) did not contain any free dAGEs. After gastric digestion, none of dAGEs measured were present in free form (figure 2A). Figure 2B shows the results of consecutive gastric and small intestinal digestion. Free CEL was only found in the residue, which is everything left in the TIM-1 system after $6 \mathrm{~h}$ that did not reach the effluent but would naturally enter the colon. Free $\mathrm{CML}$ was recovered in the effluent after 2 h of digestion ( $9.2 \pm 3.4 \%$ of total $\mathrm{CML}$ ) (mean $\pm \mathrm{SD}$ ). Similar percentages of free-form $\mathrm{CML}$ were also found after 3 and $4 \mathrm{~h}$ of digestion. Only marginal amounts of free G-H1 was found in the samples in general (6.7 $\pm 0.9 \%$ of total $\mathrm{G}-\mathrm{H} 1$ after $3 \mathrm{~h}$ of digestion). The amount of free MG-H1 ranged from $10.9 \pm 3.6 \%$ of total MG-H1 after $2 \mathrm{~h}$ of digestion to $25.8 \pm 10.5 \%$ of total MG-H1 after $5 \mathrm{~h}$. After $6 \mathrm{~h}$ of digestion all MG-H1 found was present in the free form $(1.4 \pm 0.3 \mu \mathrm{g})$.

In general, concentrations of protein-bound CML, G-H1, and MG-H1 were highest after 2-3 $\mathrm{h}$ of gastric digestion (figure $2 \mathrm{~A}$ ) and combined gastric and small intestinal digestion (figure 2B), while gradually decreasing afterwards. The residue contained relatively high amounts of protein-bound CML, G-H1, and MG-H1. Passive absorption of nutrients from the jejunum and ileum was assessed by continuously dialyzing the digested and dissolved low-molecular weight compounds from the jejunum and ileum compartments through hollow fiber membrane systems. No free or protein-bound dAGEs were found in the dialysate of either jejunum or ileum (data not shown). As a control, unglycated casein (UC) was used which consisted of the same components as GC but it did not undergo thermal treatment. No dAGEs were found in the UC (data not shown). 
A

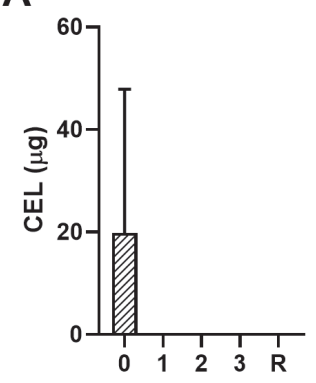

Digestion time (h)

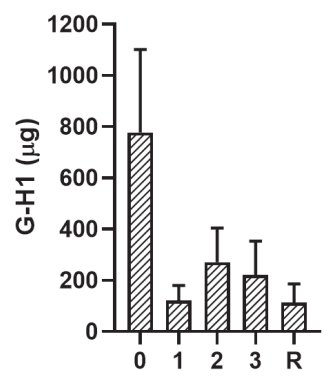

Digestion time (h)

B

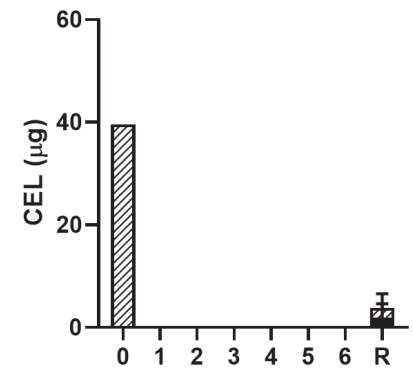

Digestion time (h)

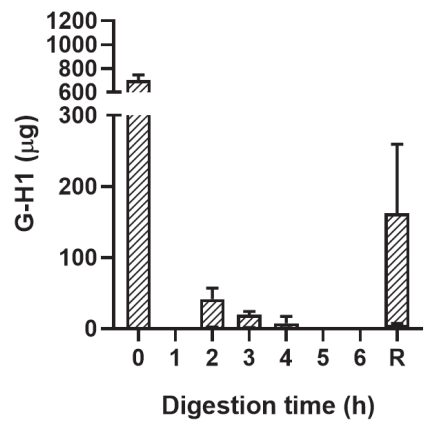

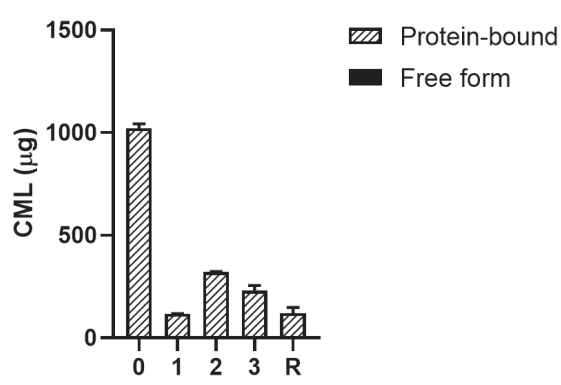

Digestion time (h)

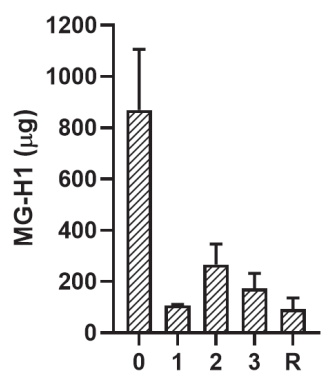

Digestion time (h)
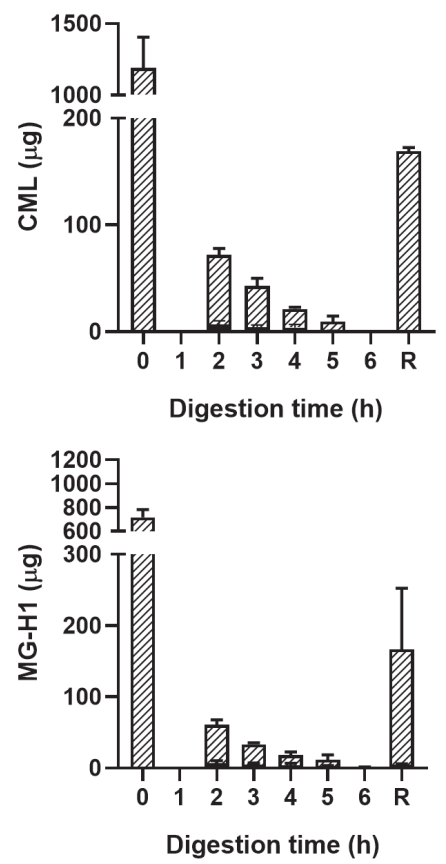

Figure 2. Free- and protein-bound CEL, CML, G-H1, and MG-H1 in the ileal efflux of GC after gastric digestion (A) and complete gastric-small intestinal digestion (B) measured by UPLC-MS/MS. Time point $0 \mathrm{~h}$ digestion is the undigested starting product. Data are presented as mean $\pm S D, n=2 . R=$ residue. Missing bars indicate that UPLC-MS/MS results were below the LOQ. 


\subsubsection{Ginger biscuit}

To confirm the findings in the food-based matrix (GC) in actual food products, a popular ginger biscuit was digested using the same regime as glycated casein. The results for gastric digestion are presented in figure $3 \mathrm{~A}$. The starting product contained small quantities of free CML ( $4.4 \pm 0.5 \%$ of total CML) (mean \pm SD) and MG-H1 (1.6 $\pm 0.4 \%$ of total MG-H1). No free CEL was found in the starting product and gastric digestion did not lead to the formation of any free $\mathrm{CEL}$. However, CML, G-H1, and MG-H1 were found in free form at all different time points of the gastric digestion period. The fractions of free vs total dAGE were highest after 1 h of gastric digestion with $12.4 \pm 2.9 \%$ of total $\mathrm{CML}, 10.7 \pm 10.8 \%$ of total $\mathrm{G}-\mathrm{H} 1$, and MG-H1 $13.3 \pm 4.3 \%$ of total MG-H1.

Figure $3 \mathrm{~B}$ shows the results for the combination of gastric and small intestinal digestion. Overall, MG-H1 concentrations (both the free and protein-bound form) in the ginger biscuit are higher than the other three dAGEs. After $2 \mathrm{~h}$ of digestion, the amounts of all free dAGEs peaked, containing $22.1 \pm 1.2 \%$ of total CEL, $12.0 \pm 0.1 \%$ of total CML, $15.7 \pm 2.1 \%$ of total $\mathrm{G}-\mathrm{H} 1$ and $24.1 \pm 2.3 \%$ of total MG-H1. After $2 \mathrm{~h}$ the amounts of both protein-bound and free dAGEs gradually decreased.

Passive absorption of protein-bound and free MG-H1 occurred in both the jejunum and ileum (figure 4), largely in protein-bound form. In absolute amounts, a total of $8.1 \pm 1.6 \mathrm{mg}$ MG$\mathrm{H} 1$ (free and protein-bound) left the small intestine in the effluent, while $12.8 \pm 0.4 \mathrm{mg}$ of MG-H1 (which is $61 \%$ of the total detected protein-bound MG-H1) was absorbed via passive diffusion. In contrast, passively absorbed G-H1 was only found in the ileum after $4 \mathrm{~h}$ and in only one sample. 
A
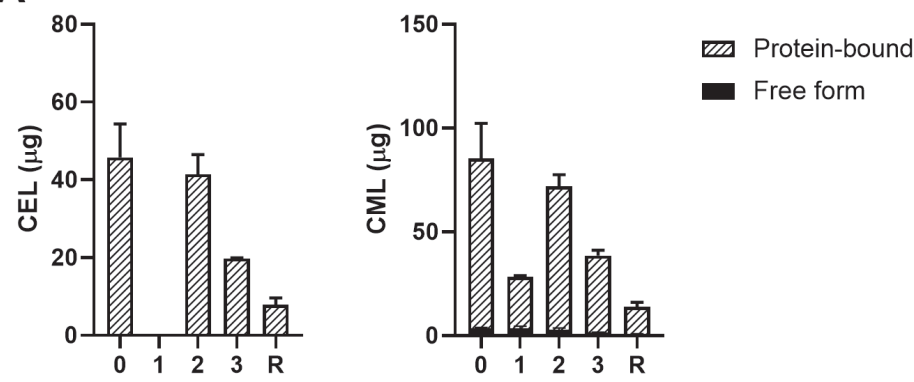

Digestion time $(\mathrm{h})$

Digestion time (h)
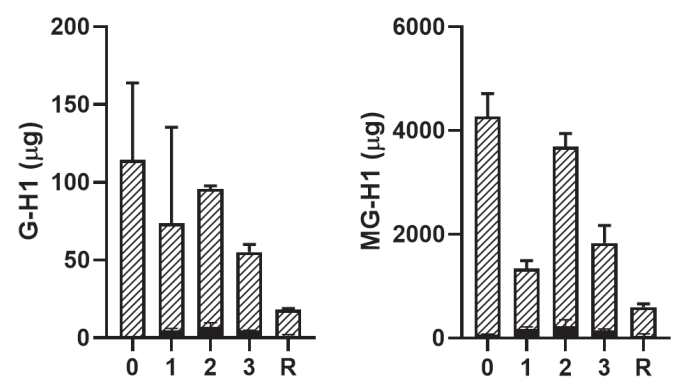

Digestion time (h)

B

Digestion time (h)

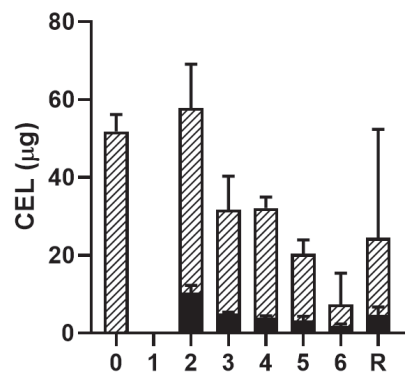

Digestion time (h)

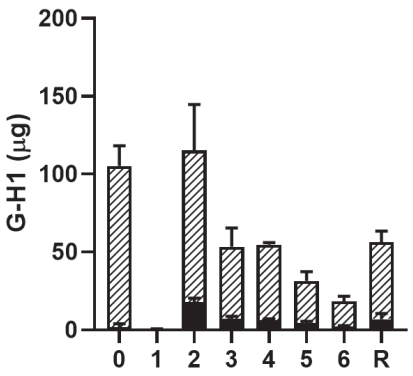

Digestion time (h)

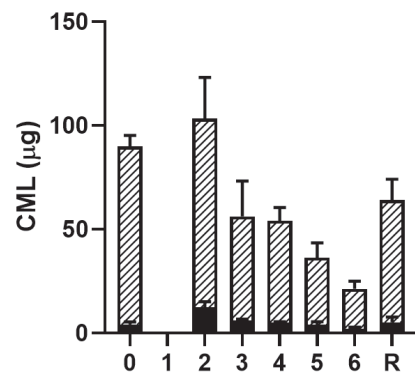

Digestion time (h)

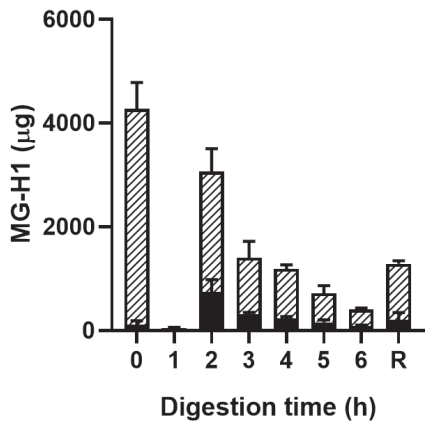

Figure 3. Free- and protein-bound $\mathrm{CEL}, \mathrm{CML}, \mathrm{G}-\mathrm{H} 1$, and $\mathrm{MG}-\mathrm{H} 1$ in the ileal efflux of ginger biscuits after gastric digestion (A) and complete gastric-small intestinal digestion (B) measured by UPLC-MS/MS. Data are presented as mean $\pm S D, n=2$. $R$ = residue. Missing bars indicate that UPLC-MS/MS results were below the LOQ. 

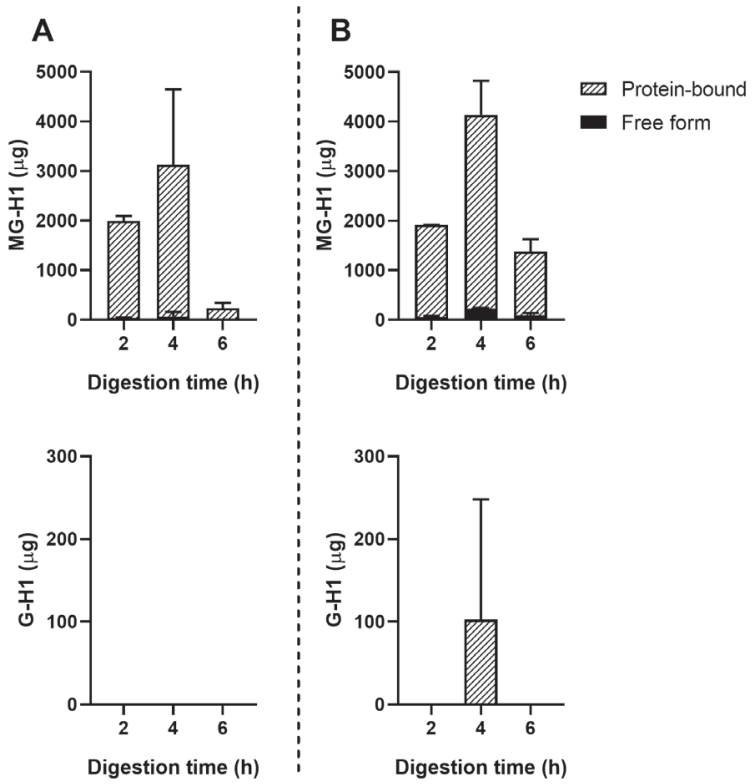

Figure 4. Passive absorption of MG-H1 and G-H1 from ginger biscuits measured by UPLC-MS/MS. (A) Jejunum dialysate. (B) lleum dialysate. Data are presented as mean \pm SD, $n=2$. Missing bars indicate that UPLC-MS/MS results were below the LOQ.

\subsubsection{Apple juice}

As an additional food product, digestion of dAGEs in apple juice was assessed (figure 5). Only free AGEs were found in the apple juice digests in varying amounts throughout gastric digestion (figure $5 A$ ). Free AGEs were also only found in the apple juice digests of the small intestine (figure 5B), albeit only for CEL and MG-H1. 
A
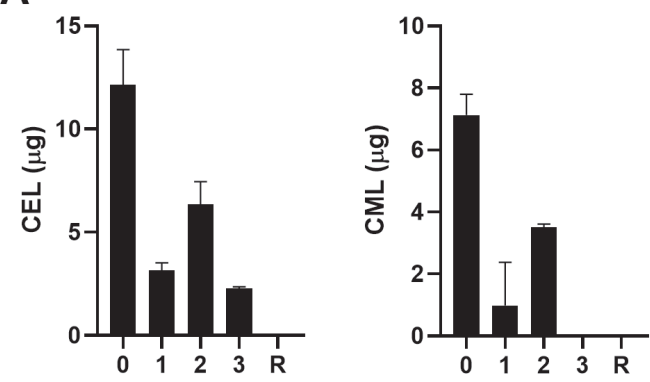

[2] Protein-bound

- Free form

Digestion time (h)

Digestion time (h)
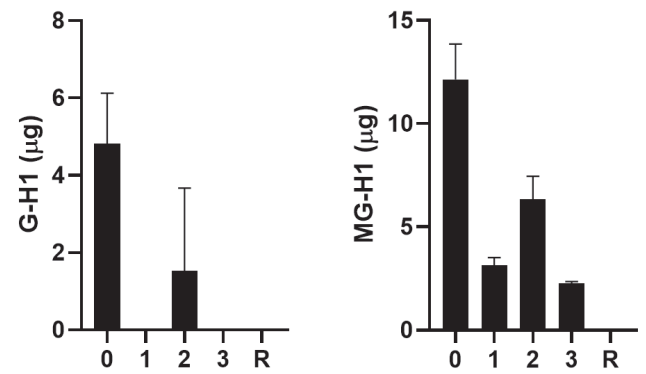

Digestion time (h)

B

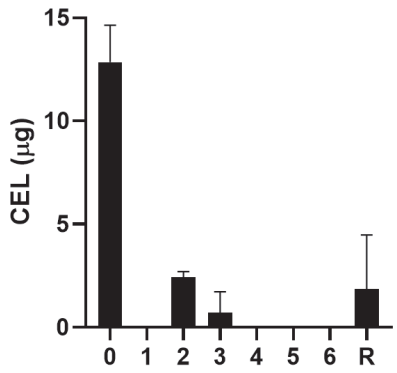

Digestion time (h)

Digestion time (h)
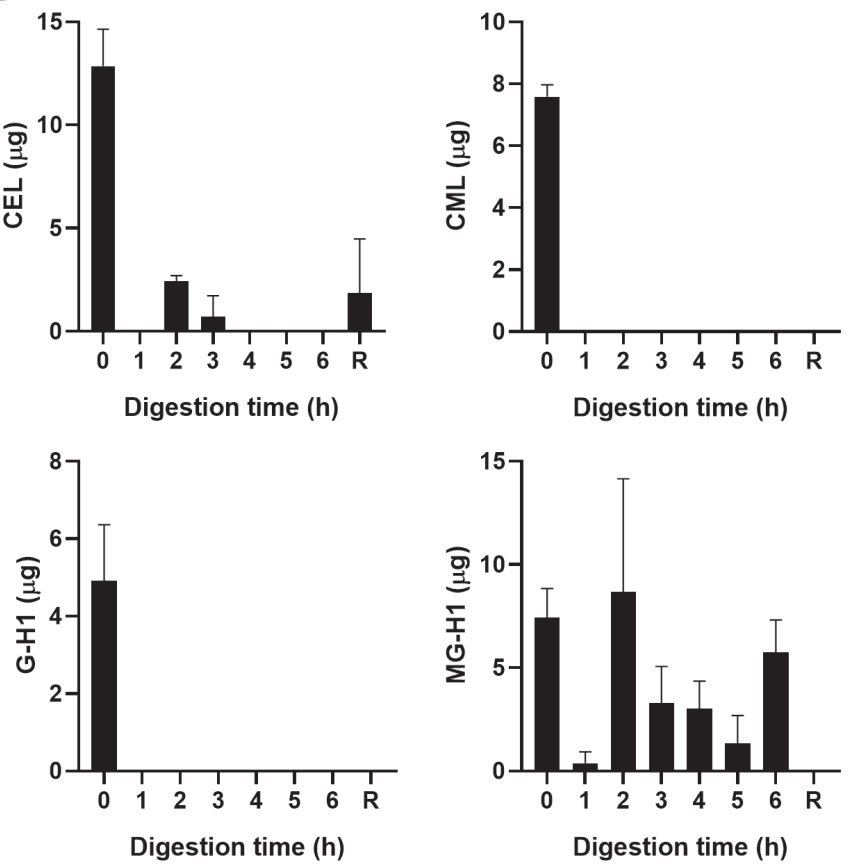

Figure 5. Free- and protein-bound CEL, CML, G-H1, and MG-H1 in the ileal efflux of apple juice after gastric digestion (A) and complete gastric-small intestinal digestion (B) measured by UPLC-MS/MS. Data are presented as mean \pm SD, $\mathrm{n}=2$. $\mathrm{R}=$ residue. Missing bars indicate that UPLC-MS/MS results were below the LOQ. 


\subsection{Digestion of glycated casein - protein digestion}

To assess the possible effects of glycation on protein digestion, the digests of GC were first separated with SDS-page and stained with the Coomassie stain. Results can be seen in figure 6. In figure $6 \mathrm{~A}$, the large band just above the $25 \mathrm{kDa}$ before digestion ( $0 \mathrm{~h}$ ) reduces during digestion, indicating a decrease in protein size. During the combination of gastric and small intestinal digestion (figure 6B) the larger proteins disappear and more smaller size proteins are present in the samples. The persistent band seen at $50 \mathrm{kDa}$ is very likely to be pancreatic lipase [17]. The difference between digestion of GC and UC was assessed by immunostaining the SDS-page membranes for bovine casein. Figure 7 shows the results for digested GC versus $U C$ in the gastric experiments. During the gastric digestion of UC, casein is only detectable in very low levels in the digests of the stomach after $2 \mathrm{~h}$ of digestion, whereas casein is still present in the digests of GC after $3 \mathrm{~h}$ of digestion. Immunostaining the small intestinal digests for casein did not show any differences (data not shown).

A

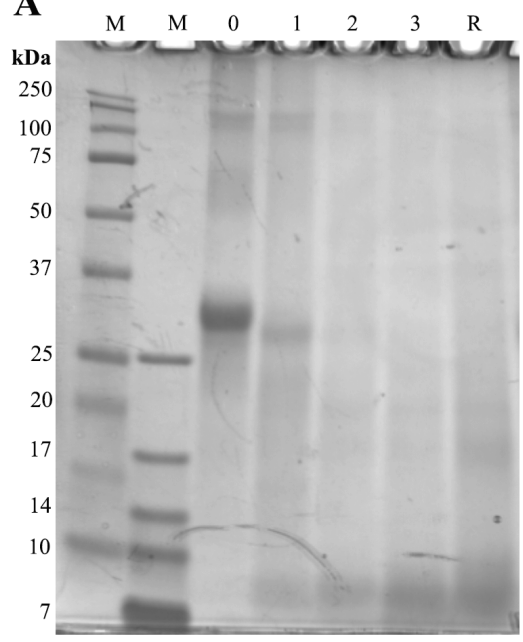

B

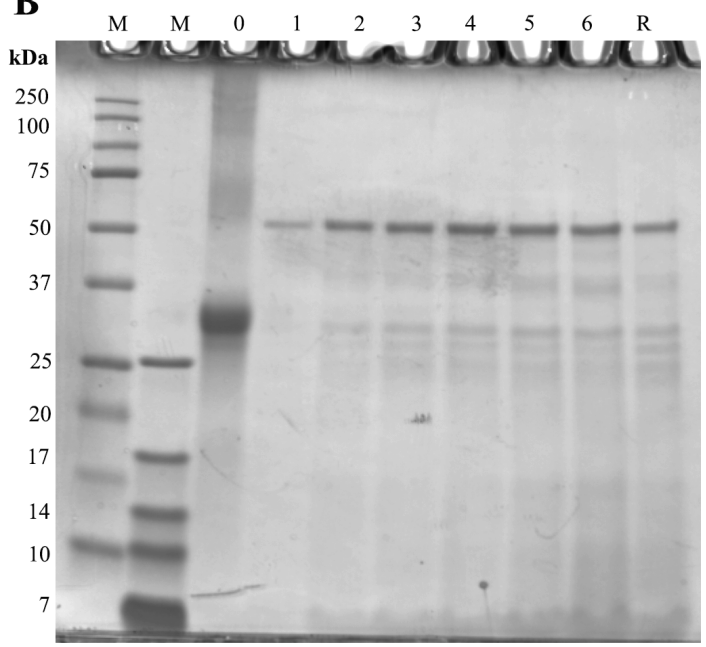

Figure 6. Protein size in digests of GC. Numbers above the gels indicate the duration ( $h$ ) of digestion and $M$ represents two different molecular markers. (A) gastric digestion. (B) gastric and small intestinal digestion. 


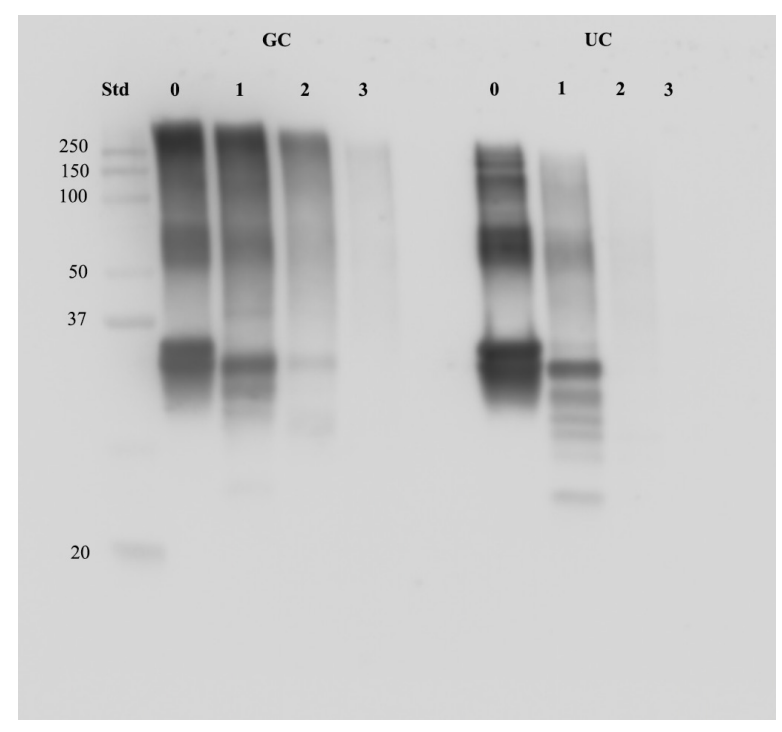

Figure 7. Protein levels of casein in GC and UC digests after $0,1,2$, and $3 \mathrm{~h}$ of gastric digestion. 


\subsection{Digestion of AGEs - recovery}

In order to calculate the absolute amounts of the different dAGEs (free and protein-bound) leaving the stomach and small intestine compartments, every effluent of the TIM-1 model was weighed to allow for a mass-balance to be made. These absolute amounts were then expressed as percentages of the total amounts of the starting products that were ingested in order to assess the recovery of dAGEs in the TIM-1 model. The results can be seen in table 1 for both total (free and protein-bound) dAGEs and only protein-bound dAGEs. For GC, the recoveries after gastric digestion for all dAGEs was $<100 \%$, and further decreased during small intestinal digestion. Recoveries for the ginger biscuit were much higher. After gastric digestion the recoveries for protein-bound dAGEs ranged from $152 \pm 13 \%$ for CEL to $231 \pm 156 \%$ for G-H1. After additional small intestinal digestion, the recoveries for proteinbound dAGEs increased even further and ranged from $276 \pm 96 \%$ for CEL to $454 \pm 73 \%$ for MG-H1. Recoveries for the free-form dAGEs in apple juice were all lower than $100 \%$ after gastric digestion. Remarkably, no CML and G-H1 and $37 \pm 25 \%$ for CEL were recovered after small intestinal digestion, whereas free MG-H1 was recovered for $296 \pm 63 \%$ after the small intestinal digestion.

\begin{tabular}{|c|c|c|c|c|c|}
\hline & & \multicolumn{2}{|c|}{ Gastric } & \multicolumn{2}{|c|}{ Small Intestinal } \\
\hline & & Total (\%) & Protein-bound (\%) & Total (\%) & Protein-bound (\%) \\
\hline \multirow[t]{4}{*}{$\mathrm{GC}$} & CEL & $0 \pm 0$ & $0 \pm 0.0$ & $5 \pm 7$ & $5 \pm 7$ \\
\hline & $\mathrm{CML}$ & $77 \pm 4$ & $77 \pm 4$ & $27 \pm 5$ & $26 \pm 5$ \\
\hline & $\mathrm{G}-\mathrm{H} 1$ & $91 \pm 13$ & $91 \pm 13$ & $33 \pm 13$ & $32 \pm 13$ \\
\hline & MG-H1 & $73.5 \pm 0.4$ & $73.5 \pm 0.4$ & $42 \pm 15$ & $38 \pm 15$ \\
\hline \multirow{4}{*}{$\begin{array}{l}\text { Ginger } \\
\text { biscuit }\end{array}$} & CEL & $152 \pm 13$ & $152 \pm 13$ & $332 \pm 104$ & $276 \pm 96$ \\
\hline & $\mathrm{CML}$ & $182 \pm 27$ & $180 \pm 30$ & $372 \pm 54$ & $350 \pm 53$ \\
\hline & $\mathrm{G}-\mathrm{H} 1$ & $247 \pm 159$ & $231 \pm 156$ & $406 \pm 221$ & $371 \pm 218$ \\
\hline & MG-H1 & $175 \pm 3$ & $163 \pm 2$ & $494 \pm 76$ & $454 \pm 73$ \\
\hline \multirow{4}{*}{$\begin{array}{l}\text { Apple } \\
\text { juice }\end{array}$} & CEL & $96.8 \pm 0.5$ & - & $37 \pm 25$ & - \\
\hline & $\mathrm{CML}$ & $62 \pm 15$ & - & $0.0 \pm 0.0$ & - \\
\hline & $\mathrm{G}-\mathrm{H} 1$ & $27 \pm 38$ & - & $0.0 \pm 0.0$ & - \\
\hline & MG-H1 & $23 \pm 33$ & - & $296 \pm 63$ & - \\
\hline
\end{tabular}

Table 1. Recovery of $\mathrm{CEL}, \mathrm{CML}, \mathrm{G}-\mathrm{H} 1$, and $\mathrm{MG}-\mathrm{H} 1$, after gastric and the combination of gastric and small intestinal digestion (noted as "small intestinal" in the table). 'Total (\%)' reflects the recoveries of both free and protein-bound dAGEs as a percentage of the total dAGEs in the undigested meal. 'Protein-bound' is the recovery of only proteinbound dAGEs as a percentage of the protein-bound dAGEs in the undigested meal. In case similar recoveries are shown for total (\%) and protein-bound (\%), this indicates that no free dAGEs were detected. Data are presented as mean $\pm S D, n=2$. 


\section{Discussion}

In this study, the digestion of the dAGE-protein-binding was assessed in a sophisticated human gastrointestinal in vitro model using a food-based matrix and actual food products. dAGEs are largely present in thermally treated food products. Earlier findings have shown that specifically protein-bound dAGEs induce a pro-inflammatory reaction in human macrophagelike cells. To what extent these protein-bound dAGEs survive the GI tract was unclear until now.

Our results show that the protein binding of dAGEs is able to survive gastric and small intestinal digestion and consequently stays intact throughout the whole Gl tract (figures 2 , 3 , and 4). These findings underline findings from earlier studies that used static digestion models, where the protein binding survived digestion. These studies were mostly focused on assessing the effect of glycation on protein digestibility. Among others, Zhao et al. (2017) saw a decrease in digestibility of $\beta$-casein after glycation with glyoxal [6]. In addition, Pinto et al. (2014) found larger aggregates of casein when casein was heated combined with glucose than when casein was heated alone. Moreover, the heated glucose-casein combination was less digestible than heated native casein [18], indicating that glycation interferes and complicates protein digestion. These results correspond to the results that were observed in the current study when comparing the digestion of GC with UC (figure 7), but only in the gastric compartment. The digestion of the casein protein was assessed with immunostaining and hampered gastric digestion of casein was seen as protein levels of casein in UC were almost completely diminished after $2 \mathrm{~h}$, whereas casein was still detectable in the digests of $\mathrm{GC}$ after $3 \mathrm{~h}$ of digestion. In the small intestines, no more differences were present between protein levels of casein in GC and UC. This was also seen by Joubran et al. (2017), who found that most peptides were liberated in the duodenum in an in vitro infant gastrointestinal tract [7]. In addition Corzo-Martinez et al. (2012) saw differences in proteolytic breakdown between different glycated proteins, so the type of protein may play a role in digestion efficiency [5]. Despite the fact that the casein protein was broken down in the small intestines, our results show that the dAGE-protein binding stays intact. As Coomassie staining and immunoblotting for casein (figure 6) showed a decrease in protein size during small intestinal digestion and break down of casein, it is possible that the dAGEs are bound to these smaller proteins or peptides after digestion.

There are several ways through which glycation can interfere with protein digestion in the small intestines. Inhibition occurs via steric hindrance of the proteolytic cleavage site by an AGE and via the direct binding of an AGE to the proteolytic cleavage site [7, 19]. Lysine and arginine contain tryptic binding sites because the $\varepsilon$-amino groups of lysine and the guanido groups of arginine are charged positively and will therefore be attracted to the negatively charged active centre of trypsin [20]. However, the same $\varepsilon$-amino group of lysine and the guanido group of arginine are major reactive sites in the Maillard reaction, which possibly explains why glycation of proteins may disrupt protein digestion. Within the group of amino 
acids, lysine is the most reactive in the Maillard reaction [21-23]. CML and CEL are lysinederived $\mathrm{dAGEs}$ and $\mathrm{MG}-\mathrm{H}$ and $\mathrm{G}-\mathrm{H}$ are arginine-derived dAGEs. By blocking the residues on these amino acids, dAGEs inhibit proteolytic digestion [20]. Interestingly, in this study a distinct effect of glycation on gastric digestion of casein rather than small intestinal digestion was observed (figure 7). The mechanism through which glycation may interfere with gastric digestion is less well known. Hydrolysis and pepsin proteolysis are the primarily responsible mechanisms for protein digestion in the stomach. The gastric $\mathrm{pH}$ plays an essential role in the activity of gastric enzymes and thus gastric digestion. A major advantage of the TIM-1 model is the dynamic $\mathrm{pH}$ range of the stomach compartment (figure S2). In the previously used static in vitro models, the $\mathrm{pH}$ is set to $2-2.5$ for two h, which poorly reflects the physiological situation as in the human stomach the $\mathrm{pH}$ is more dynamic. The fasted gastric $\mathrm{pH}$ varies between 1 - 1.5, but after ingestion, the $\mathrm{pH}$ increases to $5-7$, depending on the buffer capacity of the meal. After approximately $3 \mathrm{~h}$ of digestion, the $\mathrm{pH}$ has returned to basal levels [24]. Human gastric emptying time is of large influence on the gastric $\mathrm{pH}$, but this is also highly variable and dependent on the type of meal eaten. Sams et al. (2016) found that with liquid meals, the gastric $\mathrm{pH}$ ranges between 4 to 7 after $50 \%$ of gastric emptying, while the gastric $\mathrm{pH}$ is still 4.6 after ingestion of a solid meal and $50 \%$ gastric emptying. Pepsin exerts maximal activity at a $\mathrm{pH}$ of 2 , is still $70 \%$ active at a $\mathrm{pH}$ of 4 , but then rapidly declines in activity with increasing $\mathrm{pH}$ [25]. Denaturation of the proteins by gastric acid leads to increased accessibility of pepsin to the protein $[26,27]$. The high $\mathrm{pH}$ of the stomach in the first $h$ of digestion might explain the exit of undigested casein from the stomach into the duodenum in the UC samples; the $\mathrm{pH}$ of the stomach was not low enough for pepsin to exert its activity. The largest difference in casein digestion between GC and UC was seen in the stomach (figure 7). Opposite to lysine and arginine as tryptic cleavage sites, peptic cleavage sites include tyrosine, tryptophan, phenylalanine, and leucine. Even though these amino acids do not contain the primarily reactive residues for the Maillard reaction, all N-terminal groups of amino acids can react with 1,2-dicarbonyls. For instance, tryptophan has an indole side chain that can be glycated [28]. But since the Maillard reaction prefers lysine, the reduced digestibility that is seen in the GC vs UC gastric digestion is probably to be largely attributed to other mechanisms happening during the Maillard reaction, such as the previously discussed steric hindrance [6].

In the current study a large portion of protein bound MG-H1 in ginger biscuits was absorbed by the TIM-1 model by passive absorption through the dialysis filters (figure 4). This effect was not seen in the results of the other food products or for the other dAGEs, except one G-H1 outlier. An explanation for this can be the large concentrations of MG-H1 in the starting ginger biscuit compared to the other dAGEs. GC and apple juice also contain smaller concentrations of MG-H1. The large dilution occurring in the dialysate may have led the other dAGEs to be present in concentrations below the level of quantification of the UPLC-MS/ MS. It is however remarkable that protein-bound MG-H1 is passively absorbed to such a large extent. The physiological relevance of this finding is unclear, firstly because the method 
by which the passive absorption occurs is not very close to the real-life situation. Secondly, because previous in vitro absorption studies by Hellwig et al. (2011) showed uptake of MG$\mathrm{H} 1$ in Caco-2 cells, but strong retainment of MG-H1 in the intestinal cell [29]. It will therefore most probably not enter the systemic circulation via passive absorption.

A very interesting result of the present study was that all food products showed endogenous formation of AGEs in the small intestinal tract, with ginger biscuits showing the highest amount of endogenously formed MG-H1 represented by the recovery of more than $400 \%$ (table 1). This endogenous formation was seen in earlier studies using the static in vitro models by Martinez-Saez et al. (2019) and Bains et al. (2017) [13, 30]. Additionally, Helou et al. (2015) used the TIM-1 model to assess the digestion of fluorescent AGEs from bread crust, or soluble melanoidins as they called them, and found an increase in fluorescent properties of the soluble melanoidins [31]. The authors concluded that, since the TIM-1 model only has a temperature of $37^{\circ} \mathrm{C}$, the increase was related to "the enzymatic and chemical release of fluorescent and soluble melanoidins from larger insoluble melanoidin-skeletons". This would mean that proteins conceal glycated parts that are released during glycation. However, our method of assessing dAGE concentrations includes hydrolysing the dAGEs from their proteinbinding, circumventing this problem. We show that new AGEs are being formed and not released from their structures. Moreover, UC did not lead to any endogenous formation of AGEs, indicating that the thermal treatment that food products undergo are vital for the formation of endogenous AGEs. In this study AGEs are only formed endogenously after digestion of products that also contained AGEs, which might indicate an underlying need for the presence of AGE precursors in food, such as glyoxal and methylglyoxal. As previously stated, ginger biscuits displayed a much larger formation of food-derived endogenous AGEs than GC and apple juice. A second mechanism for the formation of endogenous AGEs in the $\mathrm{GI}$ tract could therefore be the presence of fructose in the ginger biscuit, which is highly reactive in the Maillard reaction due to its reduced stability of the ring structure compared to glucose [32]. Although conflicting evidence exists on whether there is a correlation between dAGE uptake and AGE serum and urine levels, a perspective by DeChristopher published in 2017 proposed that a source for increased serum AGE levels may be the formation of AGEs in the GI tract [33]. Fructose is actively absorbed in the human GI tract together with glucose in a 1:1 ratio. Many processed food products contain high fructose corn syrup (HFCS), making the ratio fructose:glucose $>1$, resulting in fructose malabsorption. This would leave the fructose in the Gl tract to react with proteins present. In this study a small proportion of free AGEs were seen after digestion. However, due to this additional new formation of AGEs, it is not possible to conclude whether these AGEs are liberated from their protein-binding during digestion or were newly formed. This formation of dAGEs in the intestinal tract was not seen with the food-based matrix GC, from which dAGEs were digested during gastric and small intestinal digestion. This was contrary to the study conducted by Martinez-Saez using different meal resembling systems that did show an increase in dAGEs during digestion [13]. These contradicting results can be explained by the difference in constituents of the meal 
resembling systems between this study and the one from Martinez-Saez. The nutrients used in the different studies are important for the progress of the Maillard reaction, something that is underlined in the aforementioned study. This indicates that meal resembling systems are actually poor models for food products. However, many digestion studies on dAGEs and the Maillard reaction make use of meal resembling systems. Our results show that the outcomes of these studies only using meal resembling systems should be interpreted with caution.

Gastric digestion led in both GC and apple juice to a loss of dAGEs (table 1), this implies a breakdown of dAGEs during digestion. In the case of protein-bound dAGEs in GC, the hydrolysing of the sample before UPLC-MS/MS measurement may have led to a loss of dAGES bound to peptides, since some small peptides may not have precipitated. This is not the case for the loss seen in free dAGEs in apple juice, since these samples are not hydrolysed. Another problem that arose during the UPLC-MS/MS measurement is the presence of different isomers of $\mathrm{G}-\mathrm{H}(\mathrm{G}-\mathrm{H} 1, \mathrm{G}-\mathrm{H} 2$, and $\mathrm{G}-\mathrm{H} 3)$, the isomerization may have interfered with the measurement, causing the large standard deviations seen in the absolute results of $\mathrm{G}-\mathrm{H} 1$. Another aspect that has to be taken into account is that in the current UPLC-MS/MS method a higher LOQ is obtained for the hydrolysed samples versus unhydrolysed samples. This is due to omitting the hydrolysis step in the latter approach (thereby omitting a dilution step) (Table S3). This may have resulted in a possible underestimation of the amount of protein-bound dAGEs versus free dAGEs.

The results of this study show that protein-bound dAGEs in food products stay bound to proteins during mimicked human GI digestion in both the meal resembling system and the food product. This implies for human health that dAGEs enter the human GI tract in a proinflammatory state where they might cause or aggravate inflammation. Earlier studies have shown that RAGE is upregulated in inflamed areas of the colon in Crohn's disease patients, indicating a possible role for dAGEs in inflammatory bowel diseases [34]. Glycation can also interfere with the digestion of proteins, leading to a loss of amino acids, both essential and non-essential $[6,13]$. A human study compared the protein digestibility in adolescents of a low dAGE diet and a high dAGE diet and found only a small, but significant difference. The proteins in the high dAGE diet were 6\% less digested than the low dAGE diets [35]. A more recent study assessed the effects of different glycation levels on post-prandial lysine availability in humans and saw a decrease in lysine bioavailability with increasing glycation levels [36]. This result was only seen for lysine and not for other amino acids.

In addition to having negative effects on amino acid bioavailability, some indications also exist of newly formed bioactive peptides during the Maillard reaction in foods [7]. A consequence of reduced protein and amino acid digestibility is the presence of proteins in the colon. Protein fermentation in the colon leads to the formation of detrimental compounds such as ammonia, amines, phenols and sulfides [37]. Excessive protein fermentation in the colon has been linked to colon cancer and ulcerative colitis [38]. There is no consensus on the effect of $\mathrm{dAGEs}$ in the colon and on the colonic microbiota as the effects depend on both the type of 
food product and the thermal treatment $[39,40]$.

As stated before, the $\mathrm{pH}$ of the stomach is crucial for protein digestion, as acid hydrolysis of the protein liberates proteolytic binding sites. The higher the $\mathrm{pH}$, the less access for proteolytic enzymes. People taking anti-acid medication pharmacologically elevate their stomach $\mathrm{pH}$, making acid hydrolysis less effective and thereby interfering with the digestion of proteinbound dAGEs. Additionally, age-related differences exist in stomach $\mathrm{pH}$, where the infant stomach is less acidic than the adult stomach and the Gl tract has different transit times [41, 42]. Infant formulas are extensively processed and contain protein-bound dAGEs that might have a detrimental effect on the developing infant GI tract [43]. 


\section{Conclusion}

In this study we have shown in a validated, sophisticated in vitro GI model that dAGEs enter the small intestinal and large intestinal tract in predominantly protein-bound and thereby pro-inflammatory state. Furthermore, it can be concluded that glycation hampered casein digestion. Additionally, we have shown that endogenous AGEs are formed in the GI tract with the quantities depending on the type of food product and thermal treatment. Our results show that the effect of digestion on food-based matrices, or meal-resembling systems, do not correspond to the effects on actual food products. This is important for future research into dAGEs.

\section{Acknowledgements}

We thank Jessica Verhoeven and Sanne Verhoeven at the Centre for Healthy Eating and Food Innovation (HEFI) for their valuable help with the TIM-1 model, Stanislava Vonsovic, Anouk Lentjes and Henk Gerritsen at Wageningen Food Safety Research (WFSR) for performing the UPLC-MS/MS measurements, and Casper Schalkwijk and Jean Scheijen for their feedback and suggestions during the data analysis.

This research is supported by the Dutch Food and Consumer Safety Authority (NVWA) (BAS project code WOT-02-002-004). The research was also partly funded by the Dutch Province of Limburg, with a grant to $\mathrm{HEFI}$. 


\section{References}

1. Scheijen, J., et al., Analysis of advanced glycation endproducts in selected food items by ultra-performance liquid chromatography tandem mass spectrometry: Presentation of a dietary AGE database. Food Chem, 2016. 190: p. 1145-1150.

2. Poulsen, M.W., et al., Advanced glycation endproducts in food and their effects on health. Food Chem Toxicol, 2013. 60: p. 10-37.

3. van der Lugt, T., et al., Dietary Advanced Glycation Endproducts Induce an Inflammatory Response in Human Macrophages in Vitro. Nutrients, 2018. 10(12): p. 1868.

4. Alamir, I., et al., Digestibility of extruded proteins and metabolic transit of N-epsiloncarboxymethyllysine in rats. Amino Acids, 2013. 44(6): p. 1441-1449.

5. Corzo-Martinez, M., et al., Effect of milk protein glycation and gastrointestinal digestion on the growth of bifidobacteria and lactic acid bacteria. Int J Food Microbiol, 2012. 153(3): p. 420-7.

6. Zhao, D., et al., Digestibility of Glyoxal-Glycated beta-Casein and beta-Lactoglobulin and Distribution of Peptide-Bound Advanced Glycation End Products in Gastrointestinal Digests. J Agric Food Chem, 2017. 65(28): p. 5778-5788.

7. Joubran, Y., et al., Implications of the Maillard reaction on bovine alpha-lactalbumin and its proteolysis during in vitro infant digestion. Food Funct, 2017. 8(6): p. 2295-2308.

8. Moscovici, A.M., et al., The impact of the Maillard reaction on the in vitro proteolytic breakdown of bovine lactoferrin in adults and infants. Food Funct, 2014. 5(8): p. 1898-908.

9. Pitotti, A., A. Dalbo, and M. Stecchini, Effect of Maillard Reaction-Products on Proteases Activity in-Vitro. Journal of Food Quality, 1994. 17(3): p. 211-220.

10. Guerra, A., et al., Relevance and challenges in modeling human gastric and small intestinal digestion. Trends in Biotechnology, 2012. 30(11): p. 591-600.

11. Dickinson, P.A., et al., An investigation into the utility of a multi-compartmental, dynamic, system of the upper gastrointestinal tract to support formulation development and establish bioequivalence of poorly soluble drugs. AAPS J, 2012. 14(2): p. 196-205.

12. Souliman, S., et al., A level A in vitro/in vivo correlation in fasted and fed states using different methods: applied to solid immediate release oral dosage form. Eur J Pharm Sci, 2006. 27(1): p. 72-9.

13. Martinez-Saez, N., et al., In vitro formation of Maillard reaction products during simulated digestion of meal-resembling systems. Food Res Int, 2019. 118: p. 72-80.

14. Surono, I., et al., Microencapsulation increases survival of the probiotic Lactobacillus plantarum IS-10506, but not Enterococcus faecium IS-27526 in a dynamic, computer-controlled in vitro model of the upper gastrointestinal tract. J Appl Microbiol, 2018. 124(6): p. 1604-1609.

15. Minekus, M., et al., A multicompartmental dynamic computer-controlled model simulating the stomach and small intestine. Altern Lab Anim, 1995. 23: p. 197-209.

16. Assar, S.H., et al., Determination of Nepsilon-(carboxymethyl)lysine in food systems by ultra performance liquid chromatography-mass spectrometry. Amino Acids, 2009. 36(2): p. $317-$ 26.

17. lizuka, K., et al., Purification of human pancreatic lipase and the influence of bicarbonate on lipase activity. Ann Clin Biochem, 1991. 28 ( Pt 4): p. 373-8.

18. Pinto, M.S., et al., Heating and glycation of beta-lactoglobulin and beta-casein: Aggregation and in vitro digestion. Food Research International, 2014. 55: p. 70-76.

19. van Lieshout, G.A.A., et al., How processing may affect milk protein digestion and overall physiological outcomes: A systematic review. Crit Rev Food Sci Nutr, 2019: p. 1-24.

20. Wada, Y. and B. Lonnerdal, Effects of Different Industrial Heating Processes of Milk on SiteSpecific Protein Modifications and Their Relationship to in Vitro and in Vivo Digestibility. Journal of Agricultural and Food Chemistry, 2014. 62(18): p. 4175-4185.

21. Ajandouz, E.H. and A. Puigserver, Nonenzymatic browning reaction of essential amino acids: effect of pH on caramelization and Maillard reaction kinetics. J Agric Food Chem, 1999. 47(5): p. 1786-93.

22. Kwak, E.J. and S.I. Lim, The effect of sugar, amino acid, metal ion, and $\mathrm{NaCl}$ on model Maillard reaction under pH control. Amino Acids, 2004. 27(1): p. 85-90. 
23. S. H. Ashoor, J.B.Z., Maillard Browning of Common Amino Acids and Sugars. Journal of Food Science, 1984. 49: p. 1206-1207.

24. Sams, L., et al., Relevant pH and lipase for in vitro models of gastric digestion. Food Funct, 2016. 7(1): p. 30-45.

25. Piper, D.W. and B.H. Fenton, pH stability and activity curves of pepsin with special reference to their clinical importance. Gut, 1965. 6(5): p. 506-8.

26. Berg, J.M., J.L. Tymoczko, and L. Stryer, Proteins Are Degraded to Amino Acids, in Biochemistry. 5th edition. 2002, W H Freeman: New York.

27. Schlamowitz, M. and L.U. Peterson, Studies on the optimum $\mathrm{pH}$ for the action of pepsin on "native" and denatured bovine serum albumin and bovine hemoglobin. J Biol Chem, 1959. 234: p. 3137-45.

28. Munch, G., et al., Amino acid specificity of glycation and protein-AGE crosslinking reactivities determined with a dipeptide SPOT library. Nat Biotechnol, 1999. 17(10): p. 1006-10.

29. Hellwig, M., et al., Transport of free and peptide-bound glycated amino acids: synthesis, transepithelial flux at Caco-2 cell monolayers, and interaction with apical membrane transport proteins. Chembiochem, 2011. 12(8): p. 1270-9.

30. Bains, Y., A. Gugliucci, and R. Caccavello, Advanced glycation endproducts form during ovalbumin digestion in the presence of fructose: Inhibition by chlorogenic acid. Fitoterapia, 2017. 120: p. 1-5.

31. Helou, C., et al., Insights into bread melanoidins: fate in the upper digestive tract and impact on the gut microbiota using in vitro systems. Food \& Function, 2015. 6(12): p. 3737-3745.

32. Hemmler, D., et al., Insights into the Chemistry of Non-Enzymatic Browning Reactions in Different Ribose-Amino Acid Model Systems. Sci Rep, 2018. 8(1): p. 16879.

33. DeChristopher, L.R., Perspective: The Paradox in Dietary Advanced Glycation End Products Research-The Source of the Serum and Urinary Advanced Glycation End Products Is the Intestines, Not the Food. Advances in Nutrition, 2017. 8(5): p. 679-683.

34. Ciccocioppo, R., et al., Role of the advanced glycation end products receptor in Crohn's disease inflammation. World Journal of Gastroenterology, 2013. 19(45): p. 8269-8281.

35. Seiquer, I., et al., Diets rich in Maillard reaction products affect protein digestibility in adolescent males aged 11-14 y. Am J Clin Nutr, 2006. 83(5): p. 1082-8.

36. Nyakayiru, J., et al., The glycation level of milk protein strongly modulates post-prandial lysine availability in humans. Br J Nutr, 2019: p. 1-22.

37. Gilbert, M.S., et al., Protein fermentation in the gut; implications for intestinal dysfunction in humans, pigs, and poultry. Am J Physiol Gastrointest Liver Physiol, 2018. 315(2): p. G159-G170.

38. Tuohy, K.M., et al., Metabolism of Maillard reaction products by the human gut microbiota-implications for health. Mol Nutr Food Res, 2006. 50(9): p. 847-57.

39. Snelson, M. and M.T. Coughlan, Dietary Advanced Glycation End Products: Digestion, Metabolism and Modulation of Gut Microbial Ecology. Nutrients, 2019. 11(2).

40. Perez-Burillo, S., et al., Effect of Food Thermal Processing on the Composition of the Gut Microbiota. J Agric Food Chem, 2018. 66(43): p. 11500-11509.

41. Yu, G., Q.S. Zheng, and G.F. Li, Similarities and differences in gastrointestinal physiology between neonates and adults: a physiologically based pharmacokinetic modeling perspective. AAPS J, 2014. 16(6): p. 1162-6.

42. Dupont, D., et al., Comparative resistance of food proteins to adult and infant in vitro digestion models. Mol Nutr Food Res, 2010. 54(6): p. 767-80.

43. Penndorf, I., et al., Studies on N-terminal glycation of peptides in hypoallergenic infant formulas: quantification of alpha-N-(2-furoylmethyl) amino acids. J Agric Food Chem, 2007. 55(3): p. 723-7.

44. D. Keller, R. Van Dinter, H. Cash, S. Farmer and K. Venema, Bacillus coagulans GBI-30, 6086 increases plant protein digestion in a dynamic, computer-controlled in vitro model of the small intestine (TIM-1), Benef Microbes, 2017, 8, 491-496. 


\section{Supplements A}

\section{TIM-1 model}

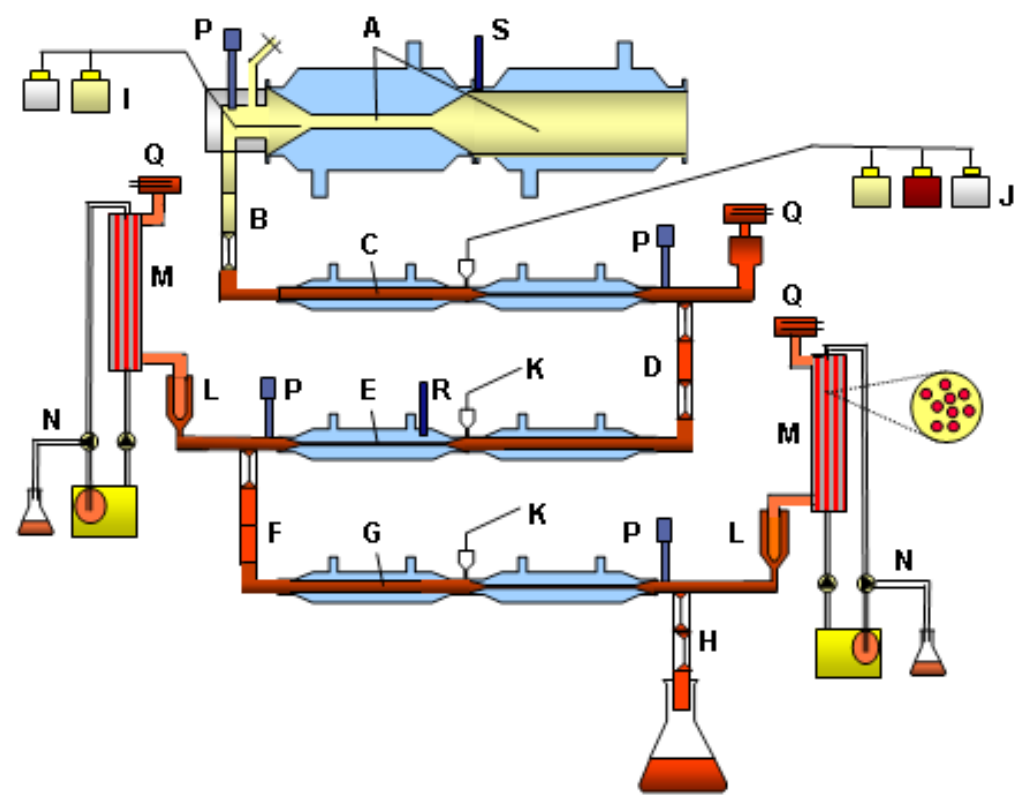

Figure S1. Schematic diagram of the dynamic, multi-compartmental TNO in vitro model of the stomach and small intestine (TIM-1). A. stomach compartment; B. pyloric sphincter; C. duodenum compartment; D. peristaltic valve; E. jejunum compartment; F. peristaltic valve; G. ileum compartment; H. ileocecal sphincter; I. stomach secretion; J. duodenum secretion; K. jejunum/ileum secretion; L. pre-filter; M. semi-permeable membrane; N. water absorption; P. pH electrodes; Q. level sensors; R. temperature sensor; S. pressure sensor. Reprinted from Keller et al. (2017) with permission [44].

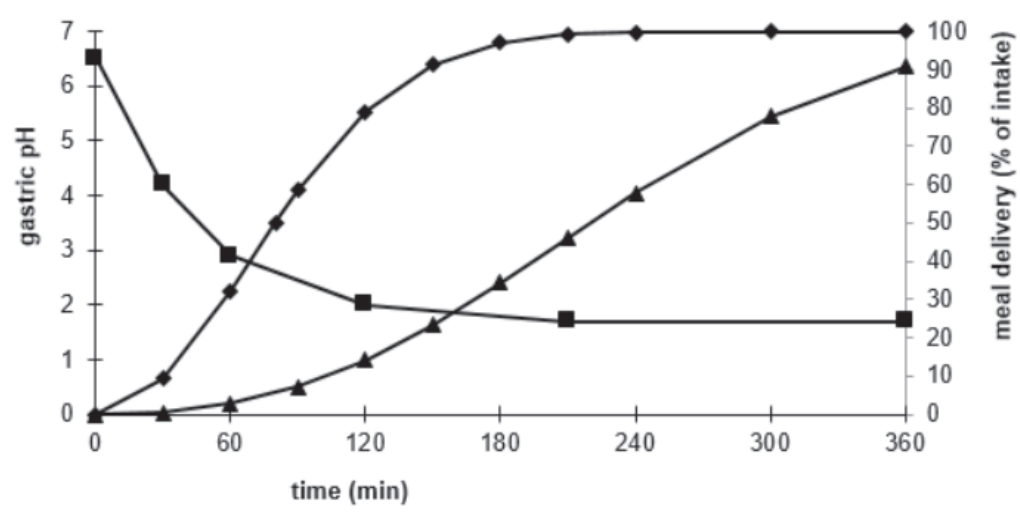

Figure S2. Gastric delivery (diamond), ileal delivery (triangle) [both expressed as percentage of the ingested meal], and the gastric $\mathrm{pH}$ (square) curves as mimicked in TIM-1 over time, representing adults. 


\section{Supplement B}

\section{Details on method of analysis for protein-bound and free dAGES}

In this study, every sample was analysed in both the hydrolysed (total dAGEs, i.e. free + proteinbound dAGEs) and unhydrolysed form (free dAGEs). The sample preparation is based on an earlier studies 1, 16 and similar to our previous study3. Briefly, sodium borate (0.33 M; pH 9) and sodium borohydride ( $2 \mathrm{M}$ ) were added to an aliquot of the samples (equivalent to $1 \mathrm{mg}$ protein) to obtain a sodium borohydride concentration of $0.2 \mathrm{M}$ in the samples. These sample solutions were incubated for $4 \mathrm{~h}$ at room temperature. Subsequently, $1 \mathrm{~mL}$ of a chloroform/ methanol (2/1 (v/v)) mix was added, and the samples were centrifuged for 10 min at 12,000 rpm. The chloroform phase was discarded and the remaining solution containing proteins was hydrolysed in $6 \mathrm{M} \mathrm{HCl}$ at $110^{\circ} \mathrm{C}$ for $18 \mathrm{~h}$. The solutions were evaporated until dryness at $50^{\circ} \mathrm{C}$ under a stream of nitrogen and dissolved in $1 \mathrm{v} / \mathrm{v} \%$ aqueous TFA ( $1 \mathrm{~mL}$ ). Prior to UPLCMS/MS analysis, internal standards and MilliQ were added to $50 \mu \mathrm{L}$ of these analyte solutions to achieve a final volume of $200 \mu \mathrm{L}$. The free dAGE content in the samples was analysed by omitting the hydrolysis step in the sample preparation procedure, thereby circumventing the release of protein-bound dAGEs. The sample extracts were analysed using an Acquity UPLC system (Waters, Milford, MA, USA) equipped with a BEH C18 analytical column $(100 \times 2.1$ $\mathrm{mm}, 1.7 \mu \mathrm{m}$ particle size, Waters, Milford, MA, USA)). The mobile phases were $5 \mathrm{mM}$ NFPA in water (A) and acetonitrile (B), and the flow was set at $0.4 \mathrm{~mL} / \mathrm{min}$. The mobile phase gradient was as follows: a linear increase from $100 \% \mathrm{~A}(\mathrm{t}=0.5 \mathrm{~min}$ ) to $70 \% \mathrm{~A}(\mathrm{t}=5 \mathrm{~min}$.) followed by a linear increase to $100 \% \mathrm{~B}(0 \% \mathrm{~A})$ at $8.5 \mathrm{~min}$, keeping $100 \% \mathrm{~B}$ to $\mathrm{t}=10.3 \mathrm{~min}$ and returning back to initial conditions again (100\% A), allowing a 2 min equilibration at these conditions. The column was kept at a constant temperature of $35^{\circ} \mathrm{C}$. A Qtrap 6500 triple quadrupole mass spectrometer (Sciex) was connected to the UPLC was operated in positive electrospray ionization (ESI+). The capillary voltage was set to $4.5 \mathrm{kV}$, and the source temperature was $500^{\circ} \mathrm{C}$, respectively. The curtain gas was set at 35 and the (instrument specific) gas $1 \& 2$ were set at 60. The injection volume was $5 \mu \mathrm{L}$. Quantification was performed using an internal standard approach and nine-point calibration curves (matrix matched standards). Quantification was performed using the precursor-product ion multiple reaction monitoring (MRM) transitions reported in below Table S1. The accuracy of the analysis was monitored by spiking each sample with a dAGEs standard. The average accuracies ranged from $75-133 \%$ (see Table S2), demonstrating that no severe losses occurred during sample preparation, and no signal enhancements or suppression occurred during UPLC-MS/MS analysis. Ion ratios between the quantifier ion and qualifier ion were monitored. In case ion ratios deviated more than $20 \%$ from the ratio observed in the standard, then the identity of the peak could not be confirmed. In the case of MG-H1 and G-H1, ion-ratio deviations were observed in GC, ginger biscuits and apple juice. A brief investigation into this issue revealed that isomers of MG-H1 (i.e. MG-H2 and MG-H3) and G-H1 (i.e. G-H2 and G-H3) may co-exist in the samples. Additional explorative experiments showed that these may co-elute and alter the ion-ratios (data not shown). Future work is needed to explore this work further, but with the isomers 
issue in consideration, deviating ion ratios were accepted for MG-H1 and G-H1. It is expected that this also played a role in the slightly elevated (or lower) accuracies for MG-H1 and G-H1 (Table S2). The limit of quantification (LOQ) is provided in Table S3. The LOQ for the protein-bound dAGEs was $125 \mu \mathrm{g} / \mathrm{L}$ and $10 \mu \mathrm{g} / \mathrm{L}$ for the free dAGEs (Table S3). This 12.5 fold difference can be explained by omitting the acid hydrolysis step, which introduces a dilution of the sample. By omitting this step, the sample is not diluted, resulting in lower LOQs.

Table S1. MS/MS settings for the UPLC analysis of dAGEs in glycated casein, ginger biscuit and apple juice.

\begin{tabular}{lllll}
\hline dAGE & $\begin{array}{l}\text { Precursor }> \\
\text { product ion }(\mathbf{m} / \mathbf{z})\end{array}$ & $\begin{array}{l}\text { Collision cell exit } \\
\text { potential }(\mathbf{C X P})\end{array}$ & $\begin{array}{l}\text { Collision energy } \\
(\mathbf{C E}) \mathbf{( e V})\end{array}$ & $\begin{array}{l}\text { Declustering } \\
\text { potential }(\mathbf{V})\end{array}$ \\
\hline \multirow{2}{*}{ CML } & $205>130$ & 6 & 17 & 51 \\
\cline { 2 - 5 } & $205>84$ & 14 & 25 & 51 \\
\hline \multirow{2}{*}{ CEL } & $219>130$ & 14 & 19 & 60 \\
\cline { 2 - 5 } & $219>84$ & 10 & 27 & 60 \\
\hline \multirow{2}{*}{ MG-H1 } & $229>114$ & 8 & 21 & 50 \\
\cline { 2 - 5 } & $229>70$ & 10 & 37 & 50 \\
\hline \multirow{2}{*}{ G-H1 } & $215>152$ & 14 & 20 & 50 \\
\cline { 2 - 5 } & $215>116$ & 8 & 19 & 50 \\
\hline
\end{tabular}

Table S2. Average (+/- SD) accuracies of spiked dAGEs standards in glycated casein, ginger biscuit and apple juice.

\begin{tabular}{llllll}
\hline Sample & & CML (\%) & CEL (\%) & MG-H1 (\%) & G-H1 (\%) \\
\hline $\begin{array}{l}\text { Glycated } \\
\text { casein }\end{array}$ & Total dAGEs & $106(13)$ & $114(7)$ & $107(21)$ & $103(23)$ \\
\cline { 2 - 6 } & Free dAGEs & $100(4)$ & $105(4)$ & $75(32)$ & $92(12)$ \\
\hline \multirow{2}{*}{$\begin{array}{l}\text { Ginger } \\
\text { biscuits }\end{array}$} & Total dAGEs & $100(5)$ & $96(5)$ & $133(50)$ & $96(8)$ \\
\cline { 2 - 6 } Apple juice & Free dAGEs & $98(4)$ & $103(4)$ & $91(37)$ & $91(12)$ \\
\cline { 2 - 6 } & Total dAGEs & $99(11)$ & $104(10)$ & $106(13)$ & $106(13)$ \\
\cline { 2 - 6 } & Free dAGEs & $102(6)$ & $101(6)$ & $116(14)$ & $101(11)$ \\
\hline
\end{tabular}

Table S3. Limit of quantification of the dAGEs in this study.

\begin{tabular}{llllll}
\hline Sample & & $\mathbf{C M L}(\boldsymbol{\mu g} / \mathbf{L})$ & CEL $(\boldsymbol{\mu g} / \mathbf{L})$ & $\mathbf{M G}-\mathbf{H} \mathbf{( \mu \mathrm { g } / \mathbf { L } )}$ & $\mathbf{G}-\mathbf{H} \mathbf{( \mu \mathrm { g } / \mathbf { L } )}$ \\
\hline \multirow{2}{*}{$\begin{array}{l}\text { Glycated } \\
\text { casein }\end{array}$} & Total dAGEs & 125 & 125 & 125 & 125 \\
\cline { 2 - 6 } & Free dAGEs & 10 & 10 & 10 & 10 \\
\hline \multirow{2}{*}{$\begin{array}{l}\text { Ginger } \\
\text { biscuits }\end{array}$} & Total dAGEs & 125 & 125 & 125 & 125 \\
\cline { 2 - 6 } & Free dAGEs & 10 & 10 & 10 & 10 \\
\hline \multirow{2}{*}{ Apple juice } & Total dAGEs & 125 & 125 & 125 & 125 \\
\cline { 2 - 6 } & Free dAGEs & 10 & 10 & 10 & 10 \\
\hline
\end{tabular}



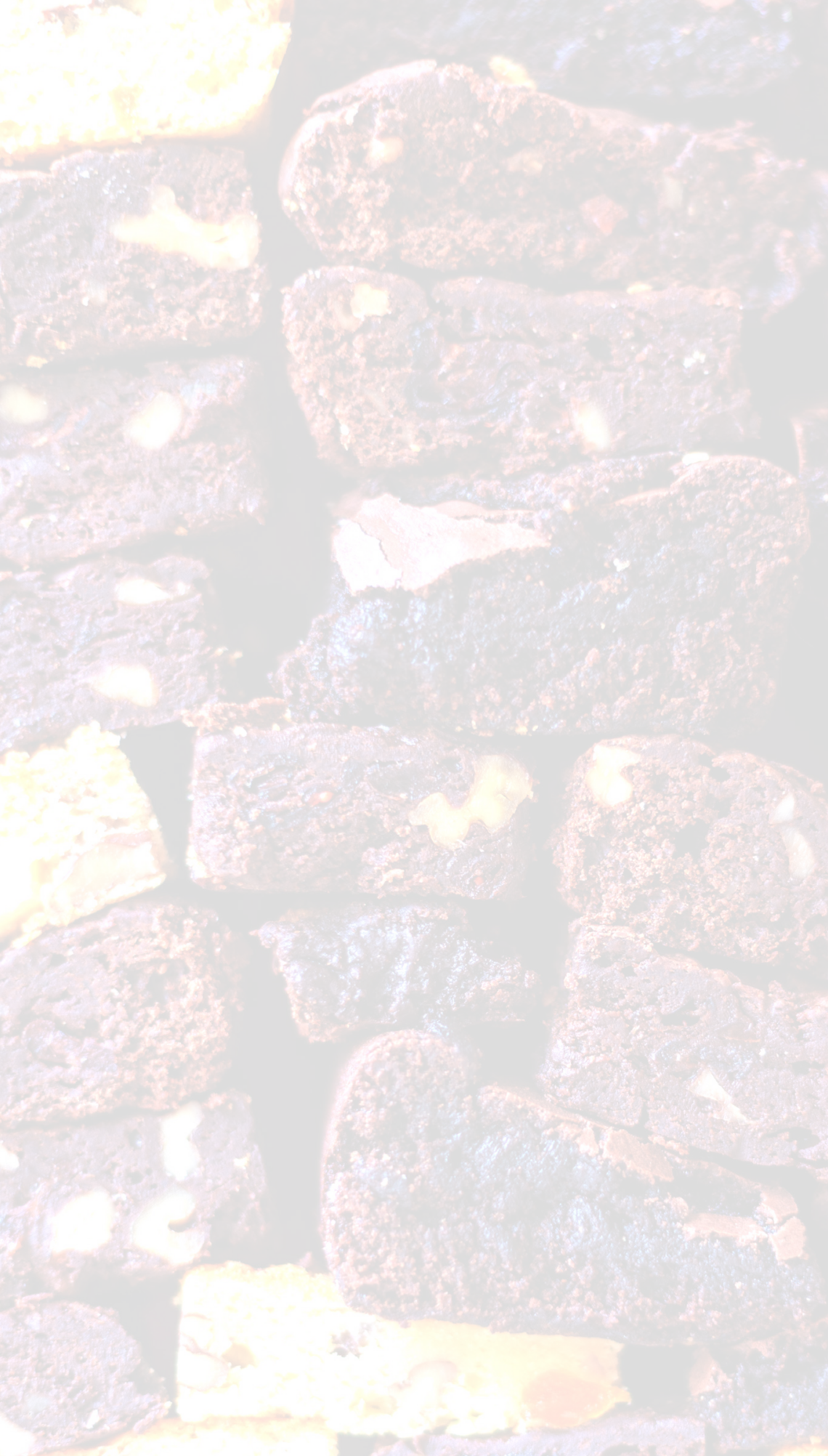


\section{Chapter 4}

\section{The pro-inflammatory potential of dietary advanced glycation endproducts after gastrointestinal digestion}

Timme van der Lugt, Misha F. Vrolijk, Antje R. Weseler, Maaike Suuring, Toine F. H. Bovee, Stefan van Leeuwen, Stanislava Vonsovic, Astrid Hamers, Antoon Opperhuizen, Aalt Bast

In preparation 


\section{Abstract}

Thermal treatment of food products leads to the formation of dietary advanced glycation endproducts (dAGEs). We have previously shown that dAGEs induce TNF- $\alpha$ secretion, cell death, and oxidative stress in human macrophage-like cells. To what extent digestion influences the pro-inflammatory effects of dAGEs is and what the implications of these proinflammatory characteristics in the human GI tract are currently unknown. In our previous study, dAGEs were digested using the TNO gastrolntestinal Model. In the current study human macrophage-like cells were exposed to both digested and undigested dAGEs and analysed for TNF- a secretion. Furthermore, the digests were fractionated and human macrophage-like cells were exposed to the different fractions to determine the effect on TNF- $\alpha$ secretion. In addition, Caco- 2 cells (gastrointestinal epithelial cells) were exposed to undigested dAGEs and assessed for cell viability and oxidative stress. The results show that digested dAGEs remain pro-inflammatory in human macrophage-like cells and that the fractions containing the larger molecules substantially contribute to this effect. The effects of dAGEs on Caco-2 cells was dependent on thermal treatment as longer treatment led to decreased cell viability. Any heat treatment of a carbohydrate-protein mixture decreased intracellular reactive oxygen species production compared to the unheated samples in these cells. In conclusion, this study shows that $d A G E s$ remain pro-inflammatory after digestion and fragmentation. These inflammatory effects seem to be both thermal treatment and cell-type dependent. 


\section{Introduction}

During thermal food processing, sugars and amino acids react with each other via the Maillard reaction, which is a series of non-enzymatic reactions, to different sub-sets of molecules. Among these molecules are Amadori products, early glycation products, advanced glycation endproducts (AGEs), and melanoidins. In diabetic patients, AGEs are also formed endogenously at $37^{\circ} \mathrm{C}$ from the reaction of carbohydrates and proteins. Endogenously formed AGEs are associated with microvascular complications in diabetes type II patients such as diabetic cardiomyopathy and peripheral neuropathies [1-3]. In previous studies we have shown that also dietary advanced glycation endproducts (dAGEs) bound to proteins, have distinct pro-inflammatory effects on human macrophage-like cells [4]. In addition, the formation of intracellular reactive oxygen species (iROS) was also induced by dAGEs in these cells [5]. Since the protein binding is essential for exerting the pro-inflammatory effect, we previously investigated the effect of human intestinal digestion on the dAGE-protein binding using the TNO Gastrolntestinal Model (TIM-1) [6]. The results of this study revealed an intact protein/peptide binding of the dAGEs and even the formation of new protein-bound dAGEs during digestion. The question that remains is if these digested dAGEs could still have proinflammatory characteristics. dAGEs exert their effects largely via activating the receptor for advanced glycation endproducts (RAGE) $[4,7,8]$. RAGE is a $35 \mathrm{kDa}$ transmembrane receptor and has a multitude of ligands. Evidence suggests that activation of RAGE is dependent on the molecular size of the ligand, hence mainly protein-bound AGEs activate RAGE. This sizedependency is likely due to oligomerization of RAGE in the cell membrane [9]. However, the specific recognition sites of RAGE, or the required ligand size for binding is currently unknown. In the current study we will therefore assess whether digestion alters the pro-inflammatory potential of glycated casein (GC), a heated food-based matrix containing a diverse set of dAGEs, in human macrophage-like cells (THP-1). Additionally, digested and undigested GC will be fractionated by size-exclusion chromatography according to molecule size, to assess the size of the pro-inflammatory molecules and their pro-inflammatory characteristics. To have a first indication of the effect of dAGEs on the gastrointestinal epithelial cells, the effect of GC on Caco-2 cells is also assessed. 


\section{Materials and methods}

\subsection{Chemicals and reagents}

Casein from bovine milk, lactose monohydrate, $\mathrm{NaOH}$, sodium-phosphate, 2-mercaptoethanol, and thiazolyl blue tetrazolium bromide (MTT) were obtained from Sigma-Aldrich (Saint Louis, MO, USA). D-glucose, glutamate, fetal bovine serum (FBS), Dulbeccco's Phospate-Buffered Saline (DPBS) were obtained from Gibco (Thermo Scientific, Waltham, MA, USA). Phorbol 12-myristate 13-acetate (PMA) were obtained from Sigma (Zwijndrecht, Netherlands).

\subsection{Preparation of glycated casein (GC) and unglycated casein (UC)}

Casein, glucose, and lactose, in the proportion of milk powder (11 mM glucose, $0.2 \mathrm{M}$ lactose, $10 \mathrm{~g} / \mathrm{L}$ casein from bovine milk: $1 \times \mathrm{GC}$ ), or two times concentrated milk powder (22 mM glucose, $0.4 \mathrm{M}$ lactose, $20 \mathrm{~g} / \mathrm{L}$ casein from bovine milk: $2 x \mathrm{GC}$ ), were suspended in $50 \mathrm{mM}$ phosphate buffer, $\mathrm{pH} 7.4$ and heated in an Erlenmeyer flask on a heating plate at $100^{\circ} \mathrm{C}$ up to 120 min. At time points 0, 15, 60, and 120 min. samples were taken and immediately cooled in ice water. Samples were aliquoted and stored at $-80^{\circ} \mathrm{C}$. Control samples of glucose, lactose and casein in the proportion of milk powder $(1 \mathrm{x} \cup \mathrm{C})$ and in the two times concentrated form $(2 x \cup C)$ were prepared without heating and were also immediately stored at $-80^{\circ} \mathrm{C}$. The levels of different $d A G E s$ are given in table S1. $1 x G C$ and the respective control $(1 x \cup C)$ samples were used in the Caco-2 experiments; $2 x G C$ and $2 x U C$ samples were used in all experiments involving THP-1 cells.

\subsection{Digestion of glycated casein}

The two times concentrated GC and UC samples were digested using the TNO gastrointestinal model (TIM-1) as described in chapter 3 [6]. In short, 300 grams of $2 x G C$ and $2 x U C$ were run through the model which comprises four connected glass compartments, representing the stomach, duodenum, jejunum, and ileum. In the model, the inner walls are squeezed, simulating peristalsis, and in each individual compartment the $\mathrm{pH}$ is measured continuously and regulated by the 'secretion' of hydrochloric acid (gastric compartment) or sodium bicarbonate (intestinal compartments). Secretion fluids (electrolytes, enzymes, bile, and pancreatic juice) were adjusted to the average concentrations as described for adults after ingestion of a meal. Pancreatic output was simulated by secreting $10 \%$ pancreatin in the small intestinal electrolyte solution. Biliary output was simulated by secreting a $2 \%$ bile (porcine bile extract) solution at $0.5 \mathrm{ml} / \mathrm{min}$. Experiments were done both in the gastric compartment only, as well as in the complete model. In the experiments in which only the gastric compartment was used, the duodenal compartment was only used for neutralization of the gastric efflux, without secretion of bile and pancreatin. This neutralization occurred before sampling. Concentrations of $\mathrm{N} \varepsilon$ - carboxymethyl-lysine (CML), $\mathrm{N} \varepsilon$-carboxyethyl-lysine (CEL), glyoxal-derived hydroimidazolone-1 (G-H1), and methylglyoxal-derived hydroimidazolone-1 (MG-H1), were assessed by UPLC-MS/MS and are shown in chapter 4. The concentrations of the samples used in this study can be found in table S1. 


\subsection{Determination of endotoxin in GC and UC samples}

The presence of endotoxin in the glycated casein samples from all time points was assessed by the commercially available PYROGENT Gel Clot LAL Assay $0.06 \mathrm{EU} / \mathrm{mL}$ sensitivity (Lonza, Basel, Switzerland). The assay was performed in accordance with the manufacturer's protocol using endotoxin-free dilutions and reagent tubes (Lonza, Basel, Switzerland).

\subsection{Size exclusion chromatography}

Undigested and digested 2xGC samples were fractionated using a size exclusion chromatography (SEC) system consisting of an Agilent 1200 series LC-UV system (Santa

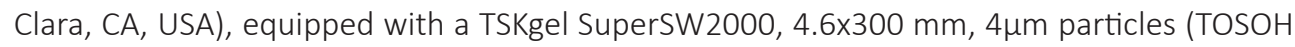
Bioscience, Griesheim (Darmstadt), Germany). The system was operated at a flow of $0.35 \mathrm{~mL} /$ min and the column was kept at a room temperature. The system was run isocratically with a 200 mM phosphate buffer at pH 6.7 (10.6 gram KH2PO and 27.2 gram Na2HPO4 x 2H2O in 1 litre demineralized water). For each run, $100 \mu \mathrm{L}$ of undiluted sample was injected. The fractionation run lasted 20 minutes and fractions of 30 seconds (equalling $0.175 \mathrm{~mL}$ ) were collected between 5 and 14 minutes. The protein size calibration was done using a standard solution of 15-600 kDa mix consisting of thyroglobulin (640 kDa), y-globulin (155 kDa), albumin $(47 \mathrm{kDa})$, ribonuclease $\mathrm{A}(13.7 \mathrm{kDa})$ and $\rho$-aminobenzoic acid $(0.14 \mathrm{kDa})$ (SigmaAldrich, St. Louis, Missouri, USA).

\subsection{Cell culture and exposure of macrophage-like cells (THP-1)}

THP-1 monocytes (ATCC, TIB-202), cultured in RPMI 1640 with L-glutamine, Hepes and phenol red (Gibco, Thermo Scientific, Waltham, MA, USA) supplemented with $10 \%(\mathrm{v} / \mathrm{V})$ fetal bovine serum (FBS), D-glucose $(4.5 \mathrm{~g} / \mathrm{L})$, Na-pyruvate $(1 \mathrm{mM})$ and 2-mercaptoethanol $(50 \mu \mathrm{M})$, were seeded in a 96 -wells plate at a cell density of 70,000 cells/well. Differentiation into macrophages was induced by adding $200 \mathrm{nM}$ of PMA to the cell culture medium and culturing them for $72 \mathrm{~h}$. Macrophage-like cells were exposed to 1 and $10 \%(\mathrm{v} / \mathrm{v})$ of $2 \times \mathrm{KC}$ and $2 x U C$ in serum and phenol-red free medium for $6 \mathrm{~h}$. Only samples that were proven to be free of endotoxins were used. Control conditions included phenol-red free medium and 1 or $10 \%$ DPBS. To investigate the effects of the SEC fractions, macrophage-like cells were exposed to $10 \%(\mathrm{~V} / \mathrm{V})$ of unfractionated and fractionated sample, respectively. Ten percent SEC diluent, as described in section 2.5 , served as a control condition.

\subsection{Cell culture and exposure of gastrointestinal epithelial cells (Caco-2)}

Caco-2 cells were cultured in DMEM containing 1\% $(\mathrm{v} / \mathrm{v})$ non-essential amino acids, $1 \%(\mathrm{v} / \mathrm{v})$ Na-pyruvate, $10 \%(\mathrm{v} / \mathrm{v})$ FBS and $4.5 \mathrm{~g} / \mathrm{ml}$ Glucose. After reaching $80 \%$ confluence, cells were transferred to a 96 -wells plate at a cell density of 20,000 cells/well. After the Caco-2 cells reached $80 \%$ confluence, the cells were exposed to final concentrations of $1 \%, 3 \%, 10 \%, 30 \%$ $(v / v) 1 \times G C$ that was heated for 15 and $120 \mathrm{~min}$ at $100^{\circ} \mathrm{C}$. To reach these final concentrations, the different $1 \times$ GC samples were suspended in DPBS and further suspended 1:1 in FBS and 
phenol-red free DMEM to which the cells were exposed for $24 \mathrm{~h}$. Control conditions included $30 \%(\mathrm{v} / \mathrm{v}) 1 \times \mathrm{xGC}$ unheated, and 30\% ( $\mathrm{v} / \mathrm{v}) \mathrm{D}$-glucose $(0.6 \mathrm{mg} / \mathrm{ml})$, lactose $(22.2 \mathrm{mg} / \mathrm{ml})$, casein $(1.5 \mathrm{mg} / \mathrm{ml})$ individually and in the combination D-glucose $(0.6 \mathrm{mg} / \mathrm{ml})+$ lactose $(22.2 \mathrm{mg} /$ $\mathrm{ml})$.

\subsection{Cell culture and exposure of Caco-2 cells to conditioned medium (CM) from macrophage-like cells}

THP-1 monocytes were cultured as described before and seeded into a 6-wells plate in a cell density of 1,050,000 cells/well. Consequently, the cells were differentiated with $200 \mathrm{nM}$ PMA for 72 h into macrophage-like cells. After 48 h, Caco- 2 cells were seeded into a 24 wells plate at a cell density of 150,000 cells/well. After the $72 \mathrm{~h}$ differentiation, macrophage-like cells were exposed to $1 \%(v / v) 2 x G C$ for $6 \mathrm{~h}$ in serum and phenol-red free RPMI 1640. Control conditions included $1 \%(\mathrm{v} / \mathrm{V})$ casein and $1 \%(\mathrm{v} / \mathrm{V})$ DPBS. After $6 \mathrm{~h}$ exposure, the medium of the macrophage-like cells was transferred to the Caco-2 cells and $1 \%$ of FBS was added to prevent the Caco- 2 cells from dying. Control conditions included the same conditions not exposed to macrophage-like cells and regular Caco-2 medium. The Caco-2 cells were then cultured for further $24 \mathrm{~h}$, after which the cell supernatant was removed and stored at $-80^{\circ} \mathrm{C}$ for ELISA analyses.

\subsection{Assessment of cell viability}

Cell viability of macrophage-like cells was assessed using the MTT (3-(4,5-dimethylthiazol-2yl)-2,5-diphenyltetrazolium bromide) assay. After removal of the cell supernatant $100 \mu \mathrm{L}$ of MTT solution $(0.5 \mathrm{mg} / \mathrm{mL}$ in DPBS) was added to each well. Then the plate was incubated in the dark at $37^{\circ} \mathrm{C}$ for $1 \mathrm{~h}$. Subsequently, the MTT solution was removed from the wells and $100 \mu \mathrm{L}$ of DMSO was added to each well. After 10 min incubation at room temperature, absorbance was measured in a microplate reader (Bio-Rad, Hercules, CA, USA) at $\lambda=540 \mathrm{~nm}$. Cell viability of Caco-2 cells exposed to CM from macrophage-like cells was assessed by a commercially available Pierce LDH Cytotoxicity Assay Kit (Thermo Scientific, Waltham, MA, USA) in accordance to the manufacturer's protocol.

\subsection{Quantification of tumor necrosis factor (TNF)- $\alpha$ in cell culture supernatants}

Tumor necrosis factor (TNF)- $\alpha$ release in cell culture supernatants of macrophage-like cells was assessed by a commercially available ELISA kit (R\&D systems, MN, USA). The assay was performed in accordance to the manufacturer's protocol.

\subsection{Quantification of intracellular ROS (iROS) levels}

iROS levels were quantified using the DCFH-DA assay. This assay measures iROS by their capacity to oxidise $2^{\prime}, 7^{\prime}$ - dichlorofluorescin diacetate (H2DCF-DA) to the fluorescent DCF [10]. In this study, Caco-2 cells were cultured and seeded into a 96 wells black plate/clear bottom according to the aforementioned methods in 2.7 and 2.8. Before exposing the cells 
to dAGEs, cells were exposed to $50 \mu \mathrm{M}$ of H2DCF-DA for $1 \mathrm{~h}$. Supernatant was removed and cells were exposed to the test conditions and controls specified in 2.7 and 2.8 for $24 \mathrm{~h}$. After $24 \mathrm{~h}$, fluorescence of DCF was measured at an excitation wavelength of $\lambda=485 \mathrm{~nm}$ and an emission wavelength of $\lambda=525 \mathrm{~nm}$ and corrected for baseline fluorescence. To calculate the relative values, fluorescence of cells exposed to only regular Caco-2 culture medium was set to $100 \%$.

\subsection{Determination of protein content of digested $2 \times G C$ and Caco- 2 cells}

Protein content of TIM-1 digests of $2 x G C$, and of Caco- 2 cells exposed to CM from macrophagelike cells in the DCFH-DA assay were assessed using the Pierce BCA Protein Assay Kit (Thermo Scientific, Waltham, MA, USA). The assay was performed in accordance to the manufacturer's protocol.

\subsection{Determination of protein content of fractionated 2xGC}

Protein content of fractionated TIM-1 digests of $2 x G C$ was assessed using the NanoDrop (Isogen Life Science, Maarsen, The Netherlands)

\subsection{Statistics}

Experiments were performed in duplicate on different days with three repetitions using the average of the duplicates as one value. Data were analysed using GraphPad Prism software (v. 5.00, GraphPad Software, San Diego, CA, USA). Data obtained were tested for normality by using the D'Agostino and Pearson omnibus normality test. Due to a lack of normal distribution, data were analysed with the nonparametric Kruskal-Wallis test followed by Dunn's Multiple Comparisons test or the Mann-Whitney $U$ test to compare two sets of treatment. The significance level was set to $p<0.05$. The significance is indicated as $* p<0.05, * * p<0.01$, and ${ }^{* * *} p<0.001$. 


\section{Results}

\subsection{Effects of digested dietary AGEs}

\subsubsection{Pro-inflammatory effect of digested dietary AGEs on human macrophage-like cells}

To assess the pro-inflammatory effects of dAGEs after GI digestion human macrophage-like cells were exposed to a $1 \%$ solution of either TIM-1 digested dAGEs or TIM-1 digested UC as a control. Digested UC, i.e. the control condition, did not lead to any TNF- $\alpha$ secretion (data not shown). Since large dilutions of the food products occur during GI digestion, the TNF- $\alpha$ secretion was corrected for the protein concentration of the different samples. The TNF$\alpha$ secretion of the cells exposed to the digested dAGEs was then expressed relative to the TNF- $\alpha$ secretion of the undigested dAGEs sample. The absolute TNF- $\alpha$ secretion is shown in figure S1. Gastric digestion of dAGEs led to an approximately $400 \%$ increase in TNF- $\alpha$ secretion, although not significant (figure 1). Prolonged GI digestion significantly increased TNF- $\alpha$ secretion more than $400 \%$. Results are displayed as relative to the undigested dAGEs (dashed line) due to large deviations between experiments. The undigested dAGEs led to 530 $\pm 252 \mathrm{pg} / \mathrm{ml}$ TNF- $\alpha$ (mean \pm SD) (figure S1).

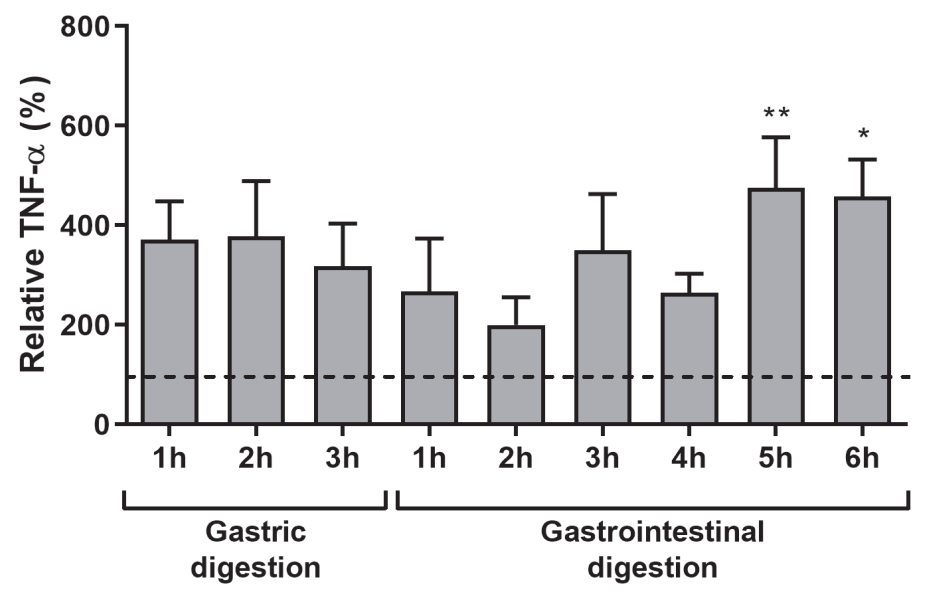

Figure 1. TNF- $\alpha$ secretion of human macrophage-like cells after $6 \mathrm{~h}$ exposure to different time points of digested dAGEs samples relative to the undigested dAGEs sample ( $0 \mathrm{~h}$ digestion) measured by ELISA and corrected for cell death (MTT) and protein content of the dAGEs samples. Dotted line represents the TNF- $\alpha$ secretion of the dAGEs prior to digestion (100\%). Data are presented as mean $\pm S D, n=3 .{ }^{*}=p<0.05,{ }^{* *}=p<0.01$ compared to the starting product. 


\subsubsection{SEC separation of undigested and digested dAGEs}

Since digested dAGEs induced a higher TNF- $\alpha$ secretion than undigested dAGEs, the undigested and digested samples were analysed for molecular size using size-exclusion chromatography. Figure 2 shows the different chromatograms for undigested dAGEs (figure $2 \mathrm{~A}$ ), $2 \mathrm{~h}$ gastric digested dAGEs (figure 2B), and $2 \mathrm{~h}$ of gastrointestinal digestion (figure $2 \mathrm{C}$ ). Figure 2D shows the size-exclusion chromatogram of different peptides and figure 2E shows 2 $h$ gastrointestinal digestion of the TIM-1 enzyme and buffer control. Separation of undigested dAGEs (figure 2A) shows a large peak at approximately $6 \mathrm{~min}$. which corresponds with protein sizes of approximately $640 \mathrm{kDa}$ (figure 2D), a second peak of sizes around $47 \mathrm{kDa}$ and then several peaks smaller than $14 \mathrm{kDa}$. Figure $2 \mathrm{E}$, the chromatogram for the enzymes and buffers used in the TIM-1 model contents, shows a peak again at $640 \mathrm{kDa}$, some molecules smaller than $47 \mathrm{kDa}$ and a peak in molecule sizes just under $14 \mathrm{kDa}$. The last peak consists of molecules smaller than $0.14 \mathrm{kDa}$. Gastric digestion (figure 2C) leads to a dramatic shift towards smaller molecule-sizes. The $640 \mathrm{kDa}$ peak has reduced by a tenth (note the difference in y-axis scale) but is probably due to the TIM-1 contents. Additional peaks arise after longer elution times that are not present in either the undigested dAGEs nor in the TIM-1 model. This indicates breakdown products of dAGEs. After $2 \mathrm{~h}$ of gastrointestinal digestion the peaks have shifted to sizes below $14 \mathrm{kDa}$.
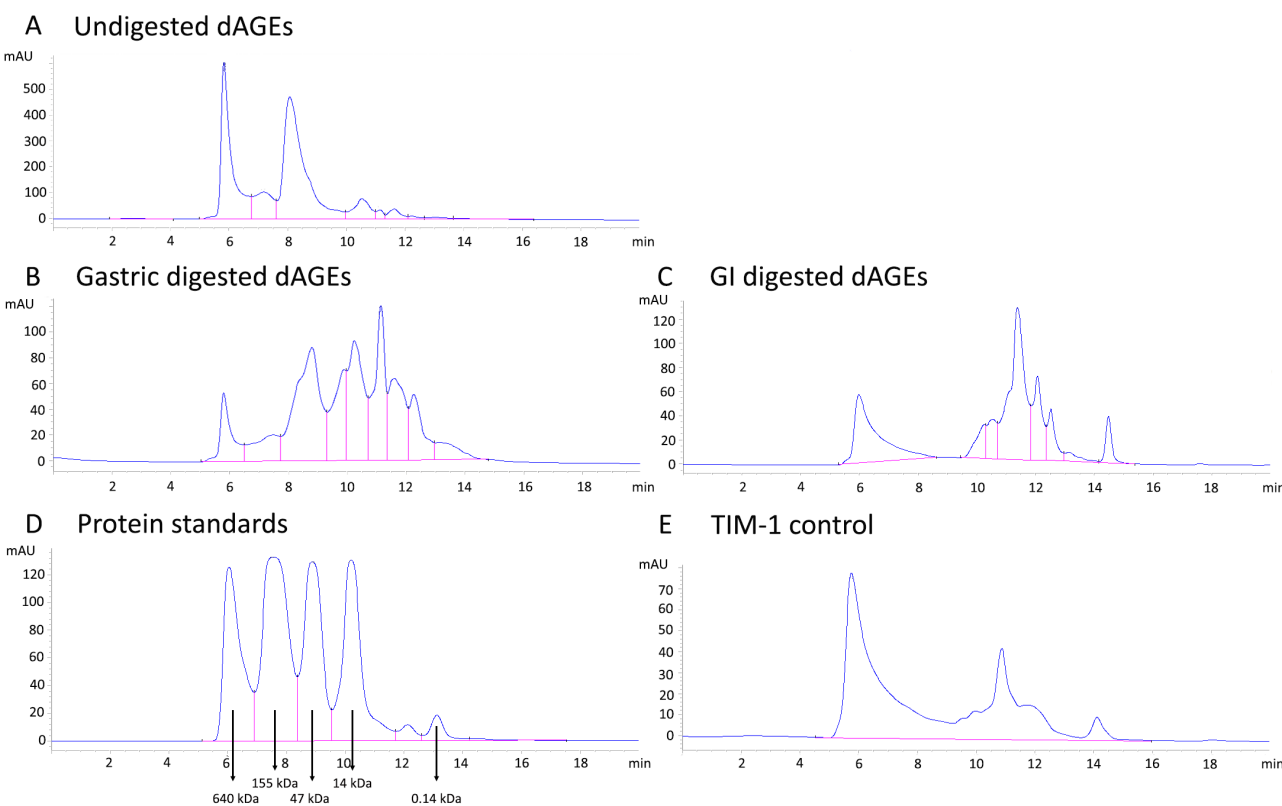

Figure 2. Size-exclusion chromatograms showing the different fractions of (A) undigested dAGEs, (B) $2 \mathrm{~h}$ gastric digested dAGEs, (C) 2 h gastrointestinal digested dAGEs, (D) protein standards consisting of thyroglobulin (640 kDa), $\gamma$-globulin $(155 \mathrm{kDa})$, albumin $(47 \mathrm{kDa})$, ribonuclease A $(13.7 \mathrm{kDa})$ and $\rho$-aminobenzoic acid $(0.14 \mathrm{kDa})$ with indicated molecular weights, (E) $2 \mathrm{~h}$ gastrointestinal digestion TIM-1 control. 


\subsubsection{Pro-inflammatory effect of fractionated undigested dAGEs on human macrophage- like cells}

In order to investigate whether the pro-inflammatory effect of undigested dAGEs can be related to a particular fraction after size exclusion chromatography, human macrophagelike cells were exposed to $10 \%(v / v)$ dilutions of these fractions. Figure $3 \mathrm{~A}$ displays the TNF- $\alpha$ concentrations in the cell supernatant per experimental condition. Absolute TNF- $\alpha$ concentrations were corrected for the TNF- $\alpha$ secretion induced by only the SEC diluent per fraction. The unfractionated sample induced the highest TNF- $\alpha$ secretion. Exposing the cells to the first fraction did not lead to any TNF- $\alpha$ secretion, fraction 2 induced some TNF- $\alpha$ secretion $(3.4 \pm 1.9 \mathrm{ng} / \mathrm{ml}($ mean \pm SD) ) and fraction 3 induced $9.6 \pm 1.3 \mathrm{ng} / \mathrm{ml}$ release of TNF- $\alpha$ from the macrophage-like cells. From fraction 4 on TNF- $\alpha$ secretion decreased until fraction 13 , after which no TNF- $\alpha$ secretion occurred anymore.

Since we have shown that only the protein-bound dAGEs are responsible for the proinflammatory response of macrophages, the protein concentration of each fraction was determined and the TNF- $\alpha$ release per mg protein was calculated (figure 3B). Since the unfractionated dAGEs have a high protein content compared to the fractions, it shows a relatively low TNF- $\alpha$ secretion per mg of protein compared to fractions 2 until 13. Fraction 2 greatly induced TNF- $\alpha$ secretion compared to both the unfractionated $1190 \pm 670 \%$ and the other fractions. Compared to the unfractionated sample this increase was $1190 \pm 670 \%$. Unfortunately due to a large standard deviation this increase is not statistically significant. Fractions 3 up until 13 induced approximately the same amount of TNF- $\alpha$ secretion, up to $\pm 500 \%$ of the unfractionated sample. The digested dAGEs were assessed in the same experiment, but the TIM-1 control (without dAGES), already induced similar levels of TNF- $\alpha$ secretion as the digested dAGEs (data not shown).

A

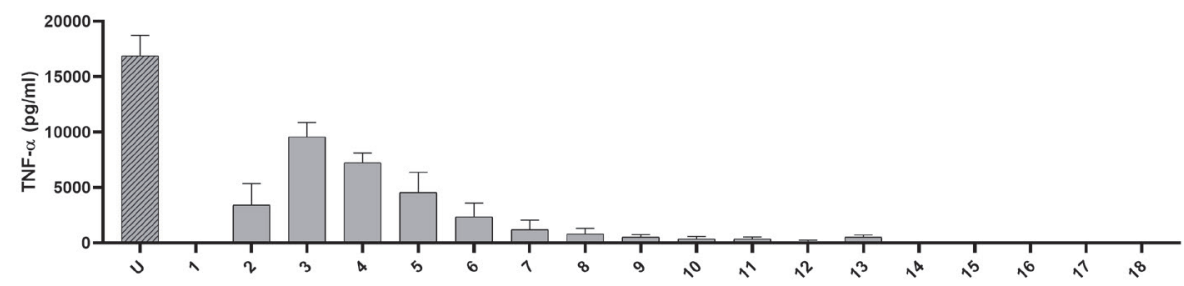

B

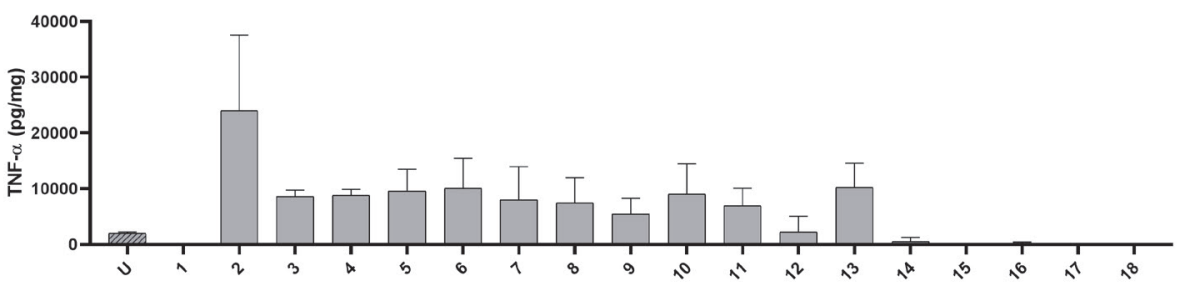

Figure 3. TNF- $\alpha$ secretion of human macrophage-like cells measured by ELISA after $6 \mathrm{~h}$ exposure to the different fractions of undigested dAGEs separated according to molecular size (1>18). " $U$ " is the unfractionated sample. (A) Absolute concentration of TNF- $\alpha$ in $\mathrm{pg} / \mathrm{ml}$ cell supernatant, (B) TNF- $\alpha$ secretion expressed in $\mathrm{pg} / \mathrm{mg}$ protein content of the undigested dAGE fractions. Data are presented as mean $\pm S D, n=3$. 


\subsection{Effects of dAGEs on gastrointestinal epithelial cells.}

To investigate the effects of dAGEs on human gastrointestinal epithelial cells, the same 1xGC samples were used as previously published [4], with two different heating times to explore whether the duration of heating changes the biological effects of the dAGEs. The concentrations of CML, CEL, and MG-H1 in the samples are shown in table S1. The TIM-1 digested samples could not be used since Caco-2 cells did not react well to the GI enzymes of the TIM-1 model.

\subsubsection{Effects of dAGEs on cell viability of human gastrointestinal epithelial cells}

Exposing Caco-2 cells to different concentrations of dAGEs heated for $15 \mathrm{~min}$. did not affect cell viability, neither did the individual components (casein, glucose, lactose and the combination UC) used to produce dAGEs (figure 4). In contrast, the dAGEs heated for 120 min. significantly reduced the cell viability by $77 \pm 15 \%$ (mean \pm SD) $(p<0.01)$ in the $30 \%(v / v)$ dilution compared to the same dilution of UC and $86 \pm 25 \%(p<0.01)$ compared to the same dilution of dAGEs heated for $15 \mathrm{~min}$. Further dilution of the $120 \mathrm{~min}$. heated dAGEs samples removed the cell viability compromising effects.

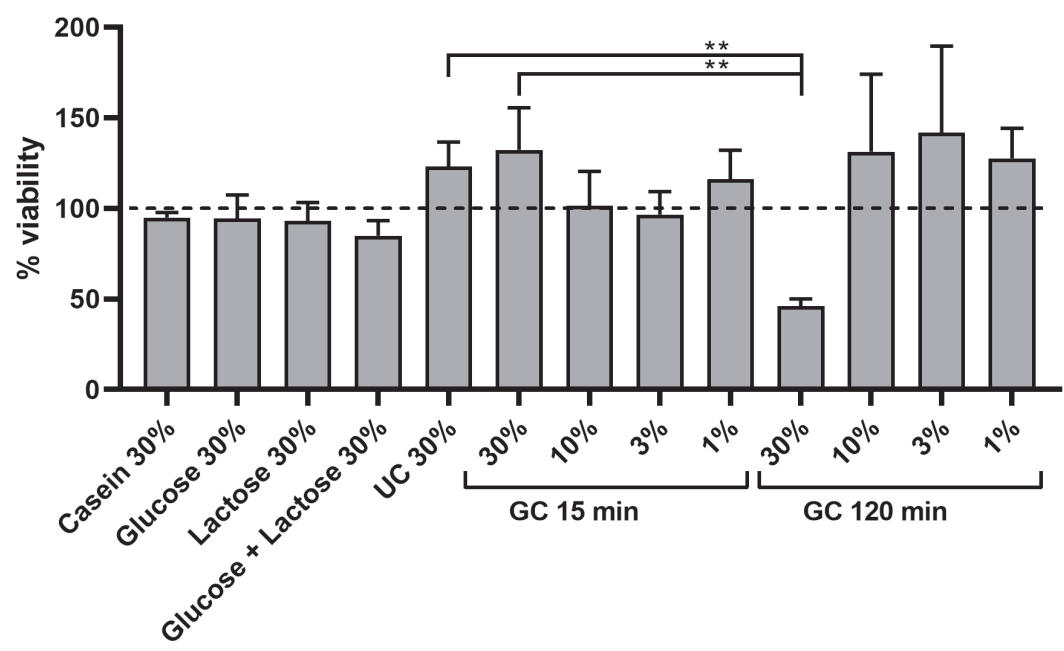

Figure 4. Cell viability of Caco-2 cells assessed by the MTT assay after $24 \mathrm{~h}$ exposure to dAGEs heated during the manufacturing process for either 15 (GC $15 \mathrm{~min}$ ) or 120 minutes (GC $120 \mathrm{~min}$ ), UC, and the different individual components of the carbohydrate-protein mixture used to prepare dAGEs. Results were normalized to control (50\% $(v / v)$ DPBS), which was set to $100 \%$ (dotted line). Data are presented as mean $\pm S D, n=3, * *=p<0.01$ 


\subsubsection{Effects of dAGEs on iROS production of human gastrointestinal epithelial cells}

The effects of different dilutions of dAGEs heated for 15 and $120 \mathrm{~min}$, and the individual components used to prepare dAGEs on iROS production of Caco- 2 cells are shown in figure 5 . Twenty four hours exposure to $30 \%(\mathrm{v} / \mathrm{V})$ casein and UC increased iROS production, although not significant compared to the vehicle control (50\% ( $/ \mathrm{v})$ DPBS). iROS production of cells exposed to UC 30\% was significantly increased compared to 30\% GC heated for 120 minutes $(p<0.001)$.

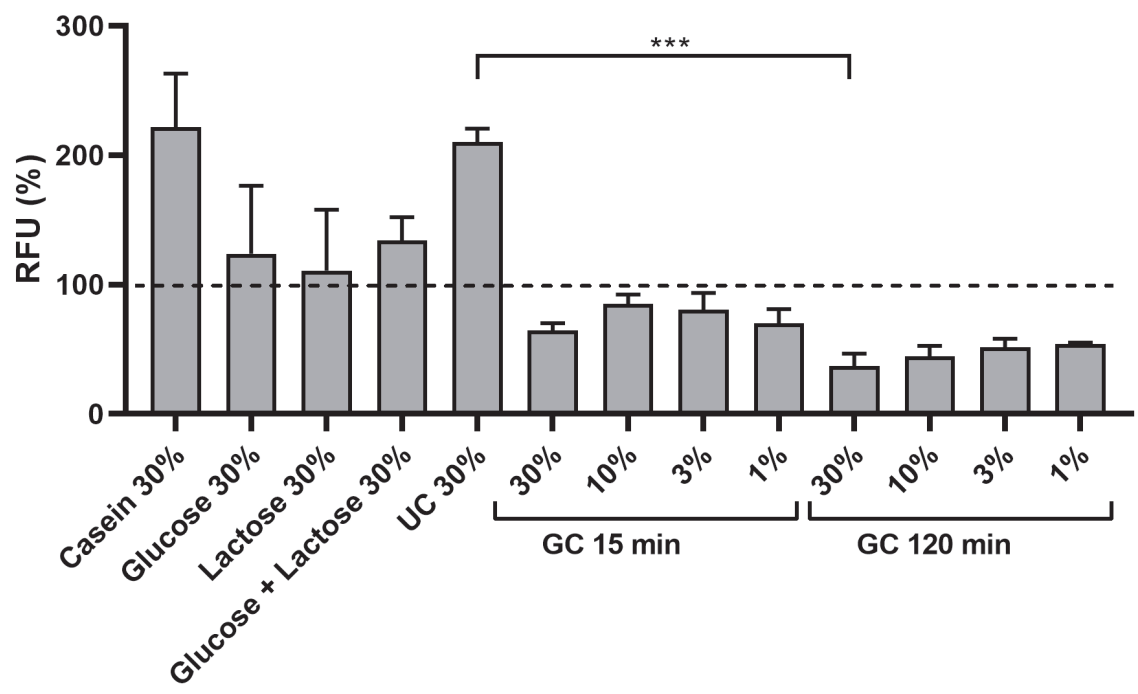

Figure 5. iROS levels of Caco-2 cells after $24 \mathrm{~h}$ exposure to dAGEs heated for either 15 (GC 15 min) or 120 minutes (GC $120 \mathrm{~min}$ ) and unglycated casein (UC, which consists of casein, lactose, and glucose unheated), and the different, unheated individual components of the mixture. The vehicle control (50\% ( $/ \mathrm{v}$ ) DPBS) was set to $100 \%$ (dotted line). Data are presented as mean $\pm S D, n=3, * * *=p<0.001$. 
3.3 Interplay between macrophage-like cells exposed to dietary AGEs and human gastrointestinal epithelial cells.

To investigate a possible interplay between activated macrophages and Caco-2 cells by dAGEs. Human macrophage-like cells were exposed to a $1 \%(v / v)$ dilution of undigested dAGEs ( $2 x G C), 1 \%(v / v)$ casein as control, and the vehicle control which consisted of $1 \%$ DPBS. After $6 \mathrm{~h}$ exposure, the macrophage conditioned medium (CM) was removed and transferred to Caco-2 cells after which they were incubated for $24 \mathrm{~h}$. As control the Caco-2 cells were additionally exposed to the same incubations but without the macrophage-CM of the macrophage-like cells.

\subsubsection{Effects of macrophage-CM exposed to dietary AGEs on cell viability of human} gastrointestinal epithelial cells

Twenty four hour exposure of Caco-2 cells to the media of macrophage-like cells exposed to either $1 \%(v / v)$ dAGEs, $1 \%(v / v)$ casein or $1 \%(v / v)$ of the vehicle control revealed an increased LDH leakage compared to cells who were exposed to the various cell culture medium controls containing the different test compounds (figure 6). No differences were seen between the different macrophage-CM with vehicle control, casein and dAGEs

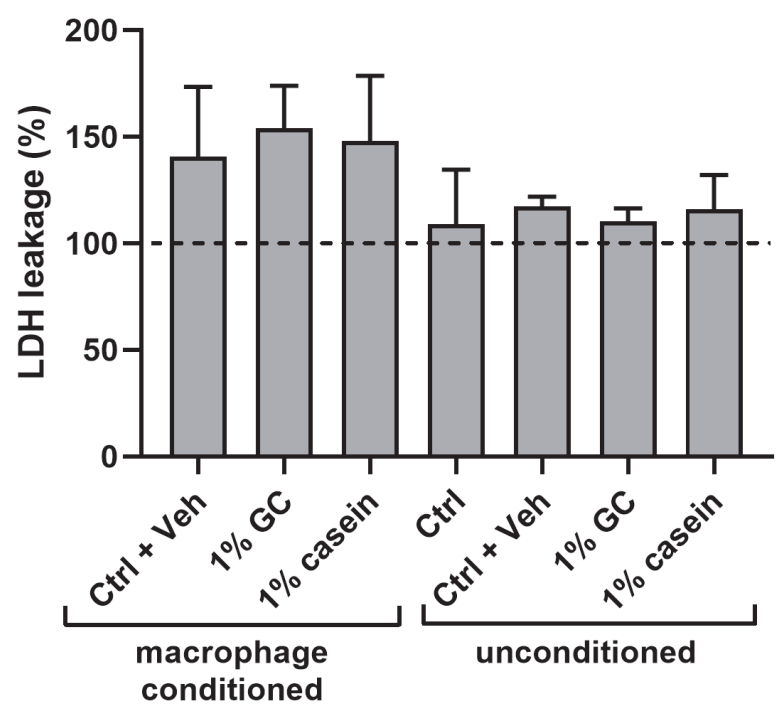

Figure 6. LDH secretion of Caco-2 cells after $24 \mathrm{~h}$ exposure to macrophage-CM exposed to either 1\% (v/v) dAGEs, 1\% $(\mathrm{v} / \mathrm{v})$ casein or $1 \%(\mathrm{v} / \mathrm{v})$ of the vehicle control or control medium including the same concentrations of dAGEs, casein or vehicle. Data are presented as mean $\pm S D, n=4$. 
3.3.2 Effects of macrophage-CM exposed to dietary AGEs on iROS formation of human gastrointestinal epithelial cells

In the presence of macrophage-CM exposed to either $1 \%(v / v)$ dAGEs, $1 \%(v / v)$ casein or 1 $\%(v / V)$ of the vehicle control (Ctrl + Veh), Caco-2 cells showed lower iROS levels compared to control cells (Ctrl) (figure 7). These cells were exposed to regular medium containing the different test compounds. Again, no differences were seen between the macrophage-CM with vehicle control, casein and dAGEs.

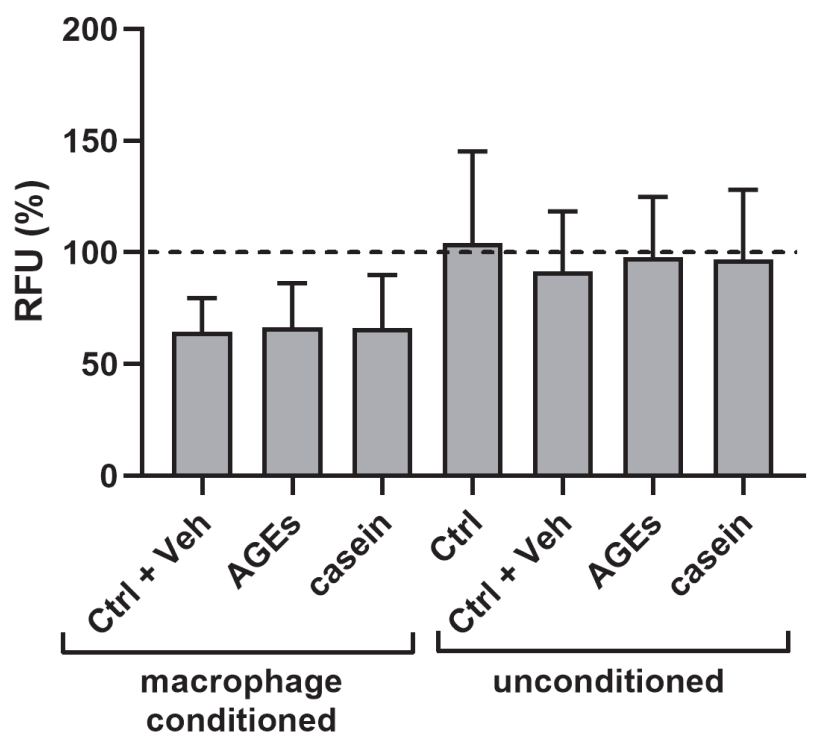

Figure 7. iROS levels of Caco-2 cells after $24 \mathrm{~h}$ exposure to macrophage-CM exposed to either $1 \%(v / v)$ dAGEs, $1 \%$ $(\mathrm{v} / \mathrm{V})$ casein or $1 \%(\mathrm{v} / \mathrm{V})$ of the vehicle control or control medium including the same concentrations of dAGEs, casein or vehicle. Baseline fluorescence of unexposed Caco-2 cells was set at $100 \%$. Data are presented as mean $\pm S D, n=4$ 


\section{Discussion}

The pro-inflammatory effects of endogenous AGEs have been well documented [1-3]. In contrast, the evidence on the pro-inflammatory potential of dAGEs is however more scarce and needs more attention. Our earlier published study revealed that also dAGEs possess proinflammatory properties, and specifically protein-bound dAGEs [4]. However, before dAGEs could exert these effects in the intestines or systemically, they undergo digestion by the GI tract. In our previous paper, we have shown that the protein-dAGE binding stays intact during GI digestion [6]. To what extent the digestive processes influence the inflammatory effects of dAGES is however unknown. In this paper, we therefore assessed the effects of dAGEs on their pro-inflammatory potential in human macrophage-like cells after $\mathrm{Gl}$ digestion and compared this to the effects of undigested dAGEs. Our results show that digested dAGEs still have a high pro-inflammatory potential as illustrated by the increasing trend on the TNF- $\alpha$ secretion compared to undigested dAGEs (figure 1). After correction for the protein content, digested dAGEs are approximately 4x more pro-inflammatory than undigested dAGEs.

Our research group has previously analysed how GI digestion influences the availability and composition (protein-bound vs free form) of dAGEs [6]. Not only were protein-bound dAGEs able to survive the GI tract, also new endogenous AGEs were formed in the intestines. During digestion, proteins are usually broken down into smaller peptides. As a consequence, smaller protein- or peptide-bound dAGE fractions could be formed in the Gl tract. These changes could potentially change the pro-inflammatory characteristics of the dAGEs. Indeed, our results show that after digestion smaller sized proteins are being formed, as shown by multiple new peaks that were detected after gastric digestion of GC (figure 2). After complete $\mathrm{GI}$ digestion, again a change in chromatogram was seen, indicating further breakdown of GC.

The present study also shows that even after digestion, dAGEs remain pro-inflammatory. In order to determine how fragmentation of protein-bound AGEs affects the pro-inflammatory effects of dAGEs, the effect of specific sizes of dAGE-protein complexes was assessed. Therefore, the dAGEs were fractionated by size-exclusion chromatography and the different fractions were tested on the induction of TNF- $\alpha$ secretion in human macrophage-like cells. After correction for protein content of the fractions, especially fractions containing molecules larger than $640 \mathrm{kDa}$ induced high levels of TNF- $\alpha$ secretion (figure 3). Fractions smaller than $1 \mathrm{kDa}$ did not induce any TNF- $\alpha$ secretion. This indicates that especially the large molecules or aggregates are responsible for the pro-inflammatory effects of dAGEs. One of the possible mechanisms by which dAGEs could induce inflammation is via the Receptor for Advanced Glycation Endproducts (RAGE). RAGE is proposed to be a pattern recognition receptor and has many different ligands next to AGEs, among which are proteins with high inflammatory potentials such as high mobility group box 1 (HMGB1), lipopolysaccharides (LPS), the signalling molecule lysophosphatic acid (LPA), damage-associated molecular patterns, and advanced oxidation protein products (AOPPs) $[8,9]$. We have previously shown that blocking RAGE 
lowers the pro-inflammatory effects of AGEs [4]. Potentially, different sizes of protein-bound dAGES might interact differently with RAGE. Oligomerization of RAGE on the cell surface is important for signal transduction, and might explain why specifically larger molecule structures bind and activate RAGE $[9,11]$. To what extent digestion of dAGEs change the binding to RAGE is unknown and therefore more research is needed to investigate whether our results here can be explained by RAGE activation. This could be achieved by blocking RAGE on the cell surface or by assessing the different fractions in a RAGE-binding assay.

Remarkably, fractionated samples showed in general higher pro-inflammatory characteristics than the unfractionated sample. A possible explanation might be the different characteristics of the molecules that are formed during the Maillard reaction. One sub-set formed, named early glycation products, have been shown in one study to exert anti-inflammatory effects by polarizing macrophages to the more anti-inflammatory $\mathrm{M} 2$ type in $\mathrm{C} 57 \mathrm{BL} / 6$ male mice to early glycation products [12]. In a study of Oh et al. (2017), Iysine-galactose Maillard reaction products (MRPs) of 3 to $10 \mathrm{kDa}$ reduced LPS-induced inflammation in RAW264.7 cells [13]. In a different study assessing the effect of MRPs on Caco-2 cells, a similar reduction of the inflammatory response was seen with small lysine-based MRPs [14]. In our experiment we found no pro-inflammatory effects of fractions smaller than $10 \mathrm{kDa}$ in size. This evidence of both, pro-inflammatory and anti-inflammatory properties of MRPs might explain why in this study the pro-inflammatory effects of the complete undigested dAGEs is less than the largest fraction, because the smaller fraction may possess anti-inflammatory properties that level out the pro-inflammatory effects. This is also exemplified by the finding that glucose-lysine MRPs downregulated NFKBIA (IKB- $\alpha$ gene) in IFN- $\gamma$ + PMA stimulated Caco- 2 cells, which suggested that inactivation of the translocation of the NF-KB subunits could be a possible mechanism for the anti-inflammatory properties [15]. However, our results show that the pro-inflammatory properties predominate the anti-inflammatory properties of MRPs in our food-based matric $2 x G C$. It is important to note that the concentrations of proteins and carbohydrates used in this study are approximately 50x lower than would be encountered when drinking a large glass of reconstituted milk powder (300 ml).

Under physiological circumstances, macrophages are not omnipresent in the GI tract. It would therefore be important to determine the local effects of dAGEs in the intestines. Currently, the effects of dAGEs on GI cells have barely been investigated. We therefore included preliminary experiments into the effects of dAGEs in this study on human GI cells. Initially, we exposed Caco-2 cells to dAGEs that underwent gastric and gastrointestinal digestion in the TIM-1 model. Unfortunately, the enzymes and secretion fluids from the TIM-1 model led to a high cell death in Caco- 2 cells and therefore it was decided to proceed with undigested dAGEs. The effect of dAGEs on the cell viability of Caco-2 cells was dependent on the preparation time, as different heating times of the carbohydrate-casein mixture led to different outcomes. Cell viability decreased significantly when cells were exposed to the highest test concentration of dAGEs heated for 120 minutes, whereas the 15 minutes heated preparation in the same 
test concentration barely influenced cell viability. Longer preparation times lead to higher levels of dAGEs and as a consequence more RAGE activation that lead to apoptosis, which might explain the decreased cell viability [8]. A similar outcome was seen in our previous study with human macrophage-like cells concerning both cell viability and TNF- $\alpha$ secretion [4]. These results indicate that in studies assessing the effects of dAGEs and MRPs in general, the type of food processing treatment defines the outcome. Similar results were found by other studies, although different compounds and effects were studies. When Pérez-Burillo et al. (2018) assessed the effects of different food products and different cooking techniques on the microbial composition and short-chain fatty acid production of human colonic bacteria, the results greatly depended on the type of food product and the cooking technique [16]. These results were underlined by the conclusions of the recent review of Snelson et al. (2019) on the effect of dAGEs on gut microbiota, in which only conflicting studies were found [17].

Since oxidative stress induces inflammation, we also investigated in this study the effects of dAGEs on the iROS production in the human gastrointestinal epithelial cell line Caco-2 [18, 19]. Both the unheated casein-lactose-glucose mixture and the casein control sample caused an increase in iROS production in Caco-2 cells. These findings suggest that the increased iROS production caused by the unheated carbohydrate-protein mix emerges from the unheated casein, as the sugar control samples did not affect the iROS levels. Although casein, and especially certain peptides, are considered to have anti-oxidative properties $[20,21]$, one study exposing peripheral blood mononuclear cells to different milk protein fractions showed an increase in iROS levels [22]. Remarkably, in our present study heating diminished this prooxidant effect in Caco-2 cells. This is in contrast to our earlier findings with macrophage-like cells, where we saw an increase in iROS levels after exposure to GC [5]. Hence, not only the biological effects of dAGEs are not only heat-treatment dependent, but also cell-type dependent.

One of the difficulties that arises when assessing the biological effects of the MRPs, is that many different sub-sets of molecules are formed during the reaction. The Maillard reaction starts with the reaction between reducing sugars and amino acids, after which many different sequential and parallel reactions lead to many different molecules, among others dAGEs [23]. Even though the name 'advanced glycation endproduct' implies that these molecules are formed at a later stage of the Maillard reaction, the actual molecules formed in the final stage are called melanoidins [24]. Melanoidins are chemically not well-defined but literature suggests that melanoidins have anti-oxidative properties, which might be one explanation for the reduction seen in iROS production after heating of the carbohydrate-protein mixture. Yáñez et al. (2017) saw an increase in anti-oxidative capacity in sugar-milk protein combinations with increasing heating time [25]. This was measured through the radical scavenging activity of the sugar-protein combinations themselves. Opposite to the large melanoidin polymers, small MRPs have been shown to possess both anti-oxidative and pro-oxidative properties, depending on the amino acid and sugar starting the reaction [13]. Oh et al. (2017) stimulated 
RAW264.7 macrophages with lipopolysaccharide (LPS) together with different sugar-amino acid MRPs. Lysine derived MRPs reduced LPS induced nitric oxide (NO) production, while arginine derived MRPs increased NO production. Especially fractions containing molecule sizes between 1 and 10 kDa of lysine derived MRPs reduced LPS-induced NO production [13]. In our experiment, the SEC data from the undigested dAGEs (figure 2A) shows the presence of molecules smaller than $10 \mathrm{kDa}$.

To mimic an inflamed gastrointestinal tract, Caco-2 cells were exposed to macrophage-CM from macrophage-like cells which were exposed to dAGEs for $6 \mathrm{~h}$. This condition has been shown to induce TNF- $\alpha$ secretion and TNF- $\alpha$ can have detrimental effects on the Gl epithelial layer, such as oxidative stress, influencing cell growth and an increase in intestinal permeability [26]. Intriguingly, we could not detect any effects on intracellular ROS production caused by the exposure of the Caco-2 cells to any of the macrophage-CM. An explanation might be that the concentration of $\mathrm{dAGE}$ and subsequent TNF- $\alpha$ secretion was too low to induce the production of ROS in the Caco-2 cells. After exposure to a $1 \%(\mathrm{v} / \mathrm{v})$ dilution of dAGEs $(2 \times G C)$, macrophage-like cells excreted approximately $530 \pm 252 \mathrm{pg} / \mathrm{ml}$ TNF- $\alpha$ (mean \pm SD) (figure S1). In a previous in vitro study, to assess the effects of TNF- $\alpha$ on monolayer permeability, Caco-2 cells were exposed to $10 \mathrm{ng} / \mathrm{ml} \mathrm{TNF- \alpha}$ to induce changes in Caco-2 intestinal permeability [26]. A different study used a concentration of $200 \mathrm{ng} / \mathrm{ml}$ TNF- $\alpha$ to induce IL-8 and IL-1 $\beta$ cytokine secretion of Caco-2 cells [27]. These concentrations are much higher than the concentrations secreted by the dAGE-exposed macrophages in our study.

In conclusion, our study showed that after GI digestion, the pro-inflammatory potential of dAGEs on cells of the innate immune response persists and even increases. Larger molecular sized protein-bound dAGEs induce more TNF- $\alpha$ release than dAGEs of lower molecular size. In addition, based on our data, it can be concluded that biological effects of dAGES on gastrointestinal epithelial cells depends on the thermal processing conditions of the dAGEs. This may be an important finding for the future assessment of dAGEs on human health. 


\section{References}

1. Pertynska-Marczewska, M., et al., Advanced glycation end products upregulate angiogenic and pro-inflammatory cytokine production in human monocyte/macrophages. Cytokine, 2004. 28(1): p. 35-47.

2. Ikeda, K., et al., N (epsilon)-(carboxymethyl)lysine protein adduct is a major immunological epitope in proteins modified with advanced glycation end products of the Maillard reaction. Biochemistry, 1996. 35(24): p. 8075-83.

3. Frati, G., et al., An overview of the inflammatory signalling mechanisms in the myocardium underlying the development of diabetic cardiomyopathy. Cardiovasc Res, 2017. 113(4): p. 378-388.

4. van der Lugt, T., et al., Dietary Advanced Glycation Endproducts Induce an Inflammatory Response in Human Macrophages in Vitro. Nutrients, 2018. 10(12): p. 1868.

5. van der Lugt, T., et al., Dietary Advanced Glycation Endproducts Decrease Glucocorticoid Sensitivity In Vitro. Nutrients, 2020. 12(2).

6. van der Lugt, T., Venema, K., van Leeuwen S., Vrolijk, M.F., Opperhuizen, A., Bast, A. , Gastrointestinal digestion of dietary advanced glycation endproducts using an in vitro model of the gastrointestinal tract (TIM-1) 2020.

7. Kierdorf, K. and G. Fritz, RAGE regulation and signaling in inflammation and beyond. J Leukoc Biol, 2013. 94(1): p. 55-68.

8. Xie, J., et al., Cellular signalling of the receptor for advanced glycation end products (RAGE). Cell Signal, 2013. 25(11): p. 2185-97.

9. Xie, J., et al., Structural basis for pattern recognition by the receptor for advanced glycation end products (RAGE). J Biol Chem, 2008. 283(40): p. 27255-69.

10. Randall, M.J., et al., The tobacco smoke component acrolein induces glucocorticoid resistant gene expression via inhibition of histone deacetylase. Toxicol Lett, 2016. 240(1): p. 43-9.

11. Moysa, A., et al., Enhanced oligomerization of full-length RAGE by synergy of the interaction of its domains. Sci Rep, 2019. 9(1): p. 20332.

12. Chen, Y. and T.L. Guo, Dietary Early Glycation Products Promote the Growth of Prostate Tumors More than Advanced Glycation End-Products through Modulation of Macrophage Polarization. Mol Nutr Food Res, 2019. 63(4): p. e1800885.

13. Oh, J.G., et al., Anti-inflammatory effect of sugar-amino acid Maillard reaction products on intestinal inflammation model in vitro and in vivo. Carbohydr Res, 2017. 449: p. 47-58.

14. Chen, X.M. and D.D. Kitts, Antioxidant and anti-inflammatory activities of Maillard reaction products isolated from sugar-amino acid model systems. J Agric Food Chem, 2011. 59(20): p. 11294-303.

15. Chen, X.M. and D.D. Kitts, Characterization of antioxidant and anti-inflammatory activities of bioactive fractions recovered from a glucose-lysine Maillard reaction model system. Mol Cell Biochem, 2012. 364(1-2): p. 147-57.

16. Perez-Burillo, S., et al., Effect of Food Thermal Processing on the Composition of the Gut Microbiota. J Agric Food Chem, 2018. 66(43): p. 11500-11509.

17. Snelson, M. and M.T. Coughlan, Dietary Advanced Glycation End Products: Digestion, Metabolism and Modulation of Gut Microbial Ecology. Nutrients, 2019. 11(2).

18. Hussain, T., et al., Oxidative Stress and Inflammation: What Polyphenols Can Do for Us? Oxid Med Cell Longev, 2016. 2016: p. 7432797.

19. Bae, H., et al., Oxidative stress-induced inflammatory responses and effects of $\mathrm{N}$-acetylcysteine in bovine mammary alveolar cells. J Dairy Res, 2017. 84(4): p. 418-425.

20. Laparra, J.M., et al., Antioxidant effect of casein phosphopeptides compared with fruit beverages supplemented with skimmed milk against $\mathrm{H} 2 \mathrm{O} 2$-induced oxidative stress in Caco2 cells. Food Research International, 2008. 41(7): p. 773-779.

21. Irshad, I., et al., Antioxidant activity of bioactive peptides derived from bovine casein hydrolysate fractions. Journal of Food Science and Technology-Mysore, 2015. 52(1): p. 231239.

22. Albenzio, M., et al., Milk nutrition and childhood epilepsy: An ex vivo study on cytokines and oxidative stress in response to milk protein fractions. J Dairy Sci, 2018. 101(6): p. 4842-4852.

23. Poulsen, M.W., et al., Advanced glycation endproducts in food and their effects on health. 
Food Chem Toxicol, 2013. 60: p. 10-37.

24. Delgado-Andrade, C. and V. Fogliano, Dietary Advanced Glycosylation End-Products (dAGEs) and Melanoidins Formed through the Maillard Reaction: Physiological Consequences of their Intake. Annu Rev Food Sci Technol, 2018. 9: p. 271-291.

25. Yáñez, D.A.C., et al., Antioxidant activity developed at the different stages of Maillard reaction with milk proteins. LWT- Food Science and Technology, 2018(89): p. 344-349.

26. Al-Sadi, R., et al., TNF-alpha modulation of intestinal epithelial tight junction barrier is regulated by ERK1/2 activation of Elk-1. Am J Pathol, 2013. 183(6): p. 1871-1884.

27. Chowers, Y., et al., Somatostatin through its specific receptor inhibits spontaneous and TNFalpha- and bacteria-induced IL-8 and IL-1 beta secretion from intestinal epithelial cells. J Immunol, 2000. 165(6): p. 2955-61. 

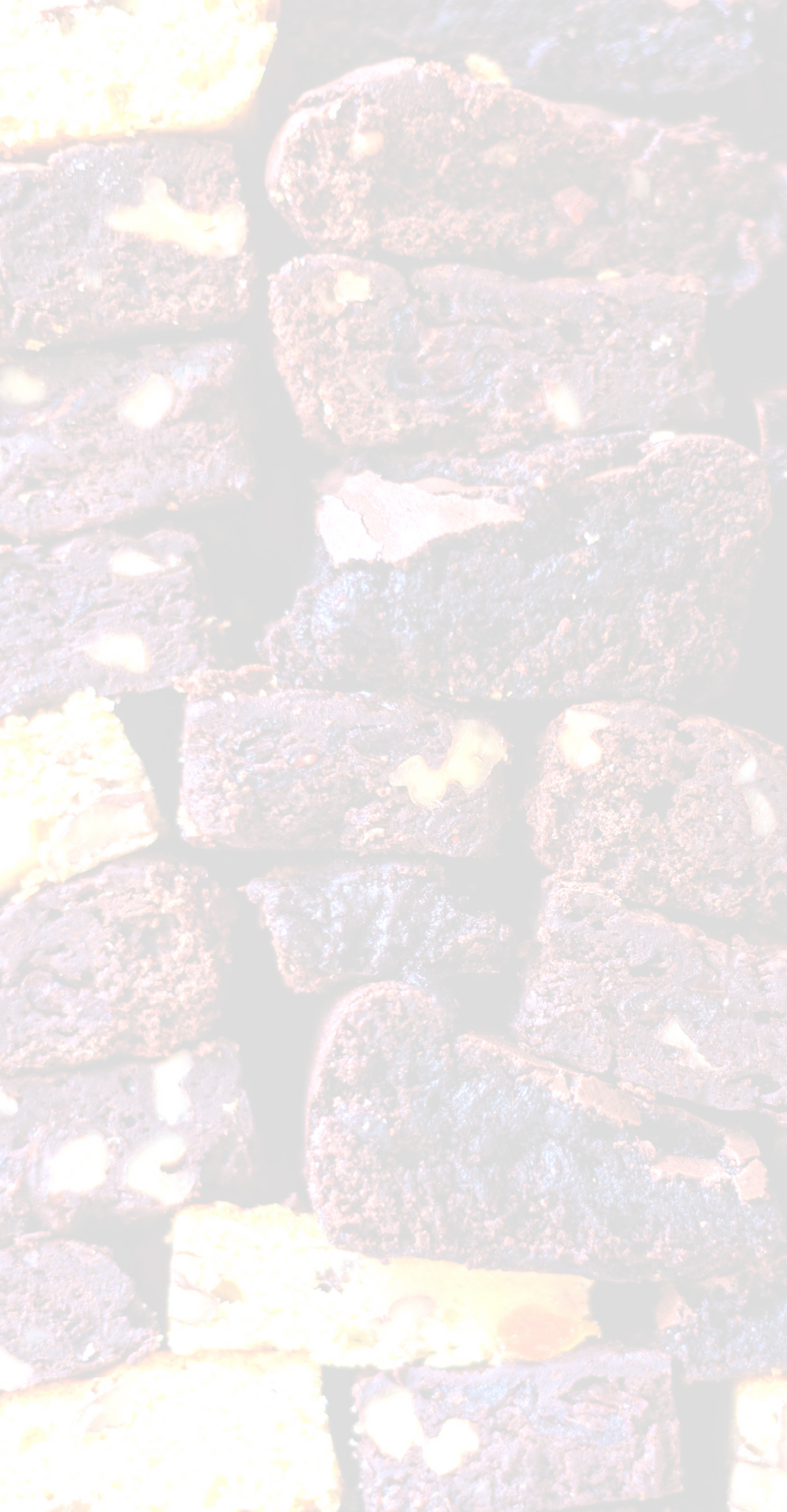

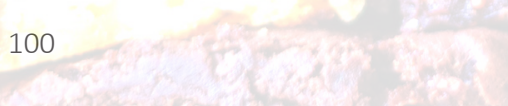




\section{Chapter 5}

\section{Dietary advanced glycation endproducts decrease glucocorticoid sensitivity in vitro}

Timme van der Lugt, Antje R. Weseler, Misha F. Vrolijk, Antoon Opperhuizen, Aalt Bast.

Nutrients, 2020 Feb 10;12(2). pii: E441 


\section{Abstract}

Glucocorticoids are very effective anti-inflammatory drugs and widely used for inflammatory bowel disease (IBD) patients. However, approximately 20\% of IBD patients do not respond to glucocorticoids and the reason for this is largely unknown. Dietary advanced glycation endproducts (AGEs) are formed via the Maillard reaction during thermal processing of food products and can induce a pro-inflammatory reaction in human cells. To investigate whether this pro-inflammatory response could be mitigated by glucocorticoids, human macrophagelike cells were exposed to both LPS and AGEs to induce IL-8 secretion. This pro-inflammatory response was then modulated by adding pharmacological compounds interfering in different steps of the anti-inflammatory mechanism of glucocorticoids: rapamycin, quercetin, and theophylline. Additionally, intracellular reactive oxygen species (ROS) were measured and glucocorticoid receptor phosphorylation state was assessed. The results show that AGEs induced glucocorticoid resistance which could be mitigated by quercetin and rapamycin. No change in phosphorylation state of the glucocorticoid receptor was observed. Additionally, intracellular ROS formation was induced by AGEs, which was mitigated by quercetin. This suggests that AGE-induced ROS is an underlying mechanism to AGE-induced glucocorticoid resistance. This study shows for the first time the phenomenon of dietary AGE-induced glucocorticoid resistance due to the formation of ROS. Our findings indicate that food products with a high inflammatory potential can induce glucocorticoid resistance, these results may be of large importance to IBD patients suffering from glucocorticoid resistance. 


\section{Introduction}

The use of glucocorticoids is increasing due to the increased prevalence of chronic inflammatory diseases such as asthma, rheumatoid arthritis and inflammatory bowel diseases (IBD). Despite the good effectiveness of glucocorticoids as anti-inflammatory drugs, some patients do not experience the anticipated therapeutic response and appear to be resistant to these drugs $[1,2,3]$. The underlying molecular mechanisms of such glucocorticoid resistance is still poorly understood.

Under physiological circumstances, glucocorticoids diffuse across the cell membrane and bind to the alpha form of the glucocorticoid receptor (GR) [4]. After binding, the GR-glucocorticoid complex translocates to the nucleus and exerts its anti-inflammatory effect in several ways. Within the nucleus, GR homodimerises and binds to glucocorticoid responsive elements (GRE) in the promotor region of glucocorticoid responsive genes, thereby switching on antiinflammatory genes [5]. These genes include genes encoding for $\beta 2$ adrenergic receptors and mitogen-activated protein kinase phosphatase-1 (MKP-1), which inhibits MAP kinases and thereby switches off pro-inflammatory gene expression [6,7]. Next to this, activated GR can increase histone deacetylation by recruiting histone deacetylase 2 (HDAC2) leading to a reduced expression of pro-inflammatory genes activated by the nuclear factor kappa-lightchain-enhancer of activated B cells (NF-KB). Lastly, glucocorticoids can also directly enhance the degradation of mRNA of pro-inflammatory genes such as that of tumor necrosis factor (TNF)- $\alpha[8,9]$.

Although the anti-inflammatory mechanisms of action of glucocorticoids are well understood, it is still unknown why glucocorticoid resistance occurs in some patients. The molecular mechanisms by which glucocorticoid resistance could arise have been reviewed by Barnes [3]. Phosphorylation of the GR seems to play an important role in causing glucocorticoid resistance. After phosphorylation of the receptor, its stability and its interaction with other proteins is altered, and nuclear translocation is decreased, thereby inhibiting its ability to bind to GRE [10-12]. Two specific proposed phosphorylation sites of the GR are serine (Ser) 226 and 211 [13-14]. These sites can be phosphorylated in several ways. For instance, the mammalian target of rapamycin (mTOR) activates C-Jun N-terminal kinase (JNK) which directly phosphorylates Ser 226 of GR. Additionally, GR can be phosphorylated through activation of p38 mitogen-activated protein kinases (p38MAPK) [14]. Compounds inducing cell stress such as reactive oxygen species (ROS) can lead to an increase in phosphoinositide 3-kinase delta (pl3Kס) and mTOR complex 1 activity, which eventually lead to less GR nuclear translocation through phosphorylation. Next, ROS can damage and thereby inactivate HDAC2 which contributes to a reduced sensitivity to glucocorticoids $[15,16]$. Hence anti-oxidants, such as the polyphenol quercetin could counteract the effects of ROS, ameliorating the sensitivity to the anti-inflammatory effects of glucocorticoids [17].

Recently, we were one of the first to show that dietary advanced glycation endproducts (AGEs) cause inflammation in human macrophages in vitro by binding to the receptor for advanced glycation endproducts (RAGE) [18]. After activation, RAGE signaling leads to the activation of 
NF-KB via PI3K and additionally to the activation of p38MAPK. Furthermore, AGEs increase the formation of intracellular ROS $[19,20]$. Therefore, we hypothesized that dietary AGEs may induce glucocorticoid resistance in human cells of the innate immune response.

Therefore, the present study aimed at investigating the effects of dietary AGEs on the antiinflammatory effects of cortisol in human macrophages and to unravel underlying molecular mechanisms by which dietary AGEs induce glucocorticoid resistance in these cells. 


\section{Materials and Methods}

\subsection{Chemicals and reagents}

Casein from bovine milk, $\alpha$-lactose monohydrate, $\mathrm{NaOH}$, sodium-phosphate, cortisol, theophylline, rapamycin, quercetin dihydrate, 2',7'- Dichlorofluorescin diacetate (H2DCFDA), 2-mercaptoethanol, and thiazolyl blue tetrazolium bromide (MTT) were obtained from Sigma-Aldrich (Saint Louis, USA). D-glucose, glutamate, fetal bovine serum (FBS), Dulbeccco's Phospate-Buffered Saline (DPBS) were obtained from Gibco (Thermo Scientific, Waltham, MA USA). Sodiumchloride, Triton-X, Tris-base, LPS and phorbol 12-myristate 13-acetate (PMA) were obtained from Sigma (Zwijndrecht, Netherlands).

\subsection{Preparation of dietary AGEs}

Dietary AGEs were prepared as described previously [18]. In short, casein, glucose and lactose, in the proportions of milk powder (11 mM glucose, $0.2 \mathrm{M}$ lactose, $10 \mathrm{~g} / \mathrm{L}$ casein from bovine milk), were dissolved in $50 \mathrm{mM}$ phosphate buffer $(\mathrm{pH}=7.4)$ and heated in an Erlenmeyer flask on a heating plate at $100^{\circ} \mathrm{C}$ up to $15 \mathrm{~min}$. After 15 minutes, a sample was taken out and put in ice water. Samples were aliquoted and stored at $-80^{\circ} \mathrm{C}$. The concentrations of several individual AGEs have been published in our previous study [18]. MG-H1 was present in concentrations of $1.6 \pm 0.9 \mu \mathrm{g} / \mathrm{ml}$ (mean \pm standard deviation (SD)), CML concentration was $0.6 \pm 0.5 \mu \mathrm{g} / \mathrm{ml}$.

\subsection{Determination of endotoxin in dietary AGEs}

Endotoxin presence in the AGE preparation was assessed by the PYROGENT Gel Clot LAL Assay with a $0.06 \mathrm{EU} / \mathrm{ml}$ sensitivity (Lonza, Basel, Switzerland). The assay was performed in accordance to the manufacturer's protocol using endotoxin-free dilutions and reagent tubes (Lonza, Basel, Switzerland). Only endotoxin-free samples were used in cell culture experiments.

\subsection{Cell culture and exposure}

THP-1 monocytes (ATCC, TIB- 202) cultured in RPMI 1640 with L-glutamine, Hepes and phenol red (Gibco, Thermo Scientific, Waltham, MA USA) supplemented with $10 \%(\mathrm{v} / \mathrm{V})$ FBS, D-glucose (4.5 g/L), Na-pyruvate (1 mM) and 2-mercaptoethanol $(50 \mu \mathrm{M})$, were seeded in a 96-wells plate in a cell density of 70,000 cells/well and differentiated into macrophages by adding $200 \mathrm{nM}$ of PMA to the cell culture medium and culturing them for $72 \mathrm{~h}$. After differentiation, the cells were exposed to $10 \%(\mathrm{~V} / \mathrm{v})$ AGEs, different concentrations of LPS, cortisol, theophylline, rapamycin, and quercetin in serum and phenol-red free culturing medium and always in a 50\% DPBS solution. Exposure concentrations of the compounds in the AGEs were: $0.16 \pm 0.09 \mu \mathrm{g} / \mathrm{ml}$ MG-H1, $0.06 \pm 0.05 \mu \mathrm{g} / \mathrm{ml} \mathrm{CML}, 1.1 \mathrm{mM}$ glucose, $20 \mathrm{mM}$ lactose and $1 \mathrm{~g} / \mathrm{L}$ casein. Control conditions included phenol-red free culturing medium and $50 \%$ DPBS. Ethanol (maximum $0.17 \mathrm{v} / \mathrm{v} \%$ ) was used as vehicle control for cortisol, theophylline and quercetin. DMSO $(0.1 \mathrm{v} / \mathrm{v} \%)$ was used as vehicle control for rapamycin. 


\subsection{Quantification of Interleukin (IL)-8 release by ELISA}

Interleukin (IL)-8 release in cell culture supernatant was assessed by a commercially available ELISA kit (R\&D systems, Minneapolis, USA). The assay was performed in accordance to the manufacturer's protocol.

\subsection{Quantification of glucocorticoid receptor protein levels by Western Blot}

THP-1 monocytes were seeded in a 6 wells plate with a cell density of 2,100,000 cells/ml and differentiated into macrophages by adding $200 \mathrm{nM}$ of PMA to the cell culture medium and culturing them for $72 \mathrm{~h}$. After differentiation, cells were exposed to 50\% DPBS, $10 \%$ $(v / v)$ AGEs and $3 \mathrm{ng} / \mathrm{ml}$ LPS for $24 \mathrm{~h}$. After incubation, cells were lysed with a Triton buffer consisting of $150 \mathrm{mM}$ sodium chloride, $0.1 \%$ Triton-X, and $50 \mathrm{mM}$ Tris $\mathrm{pH} 8$, and protein content was assessed with the BCA Assay (Thermo Scientific, Waltham, USA) according to the manufacturer's protocol.

A sample of $10 \mu \mathrm{g}$ with reducing laemmli buffer was loaded onto a $10 \%$ mini protean TGX precast gel (Bio-rad) and run in a Bio-rad cel. Protein was transferred to an Immobilon-P PVDF Membrane (Merck, Darmstadt, Germany). Membranes were analysed using Amersham Imager 600 (GE Healthcare). Primary antibodies used: Glucocorticoid Receptor (D6H2L), Phospho-Glucocorticoid Receptor (Ser211), and $\beta$-actin (13E5) from Cell Signaling Technology (Danvers, USA), Anti-phospho-Glucocorticoid receptor Ser226 from Millipore (Temecula, USA). Secondary antibody used: Anti-rabbit IgG, HRP-linked Antibody (Cell Signaling Technology).

\subsection{Quantification of ROS levels by DCFH-DA assay}

Intracellular ROS levels were quantified using the DCFH-DA assay. This assay measures intracellular ROS by their capacity to oxidise $2^{\prime}, 7^{\prime}$ - Dichlorofluorescin diacetate (H2DCF-DA) to the fluorescent DCF [21]. In this study THP-1 monocytes were cultured and seeded into a 96 wells black plate/clear bottom with PMA according to the aforementioned method. After differentiation, cells were exposed to $50 \mu \mathrm{M}$ of H2DCF-DA for $1 \mathrm{~h}$. Supernatant was removed and cells were exposed to $10 \%$ AGEs, $3 \mathrm{nM}$ cortisol and different concentrations of quercetin for 24 h. Control samples were exposed to 50\% DPBS and the vehicle control of cortisol and quercetin was ethanol. After $24 \mathrm{~h}$ fluorescence of DCF was measured at $\lambda$ excitation $=485 \mathrm{~nm}$ and $\lambda$ emission $=525 \mathrm{~nm}$, corrected for baseline fluorescence and taken as a measurement for intracellular ROS. To calculate the relative values, fluorescence of cells exposed to only regular THP-1 culture medium was taken as $100 \%$.

\subsection{Statistics}

Experiments were performed in duplicate on different days using the average of the duplicates as one value. Data were analysed using the GraphPad Prism software (v 5.00, GraphPad Software, San Diego, USA). Western Blot results were analysed using ImageJ (v1.52e, Wayne Rasband, International Institutes of Health, USA). Data obtained were tested for normality by using D'Agostino \& Pearson omnibus normality test. Data were analysed with the non- 
parametric one-way ANOVA Kruskal-Wallis test followed by Dunn's Multiple Comparison Test or Mann-Whitney $U$ test to compare two sets of treatment. Significance level was set to $p<$ 0.05. Significance is indicated as: ${ }^{*}=p<0.05 ; * *=p<0.01 ; * * * p<0.001$. 


\section{Results}

\subsection{AGE-induced glucocorticoid resistance}

Differentiated THP-1 cells were exposed to different concentrations of LPS and AGEs for 24 h, after which IL-8 secretion was assessed by ELISA and cell viability assessed by MTT. No cell death occurred after exposure to LPS and AGEs (data not shown). Cells were exposed to different concentrations of LPS and AGEs (data not shown) to test which concentrations of the different compounds lead to a similar IL-8 secretion. Figure 1 shows that exposing the macrophage-like cells to either $3 \mathrm{ng} / \mathrm{ml}$ LPS, or 10\% AGEs as eventual chosen conditions led to the same IL-8 increase. To determine the anti-inflammatory effect of corticosteroids on both AGE- and LPS-induced inflammation, cells were exposed in an additional experiment to different concentrations of cortisol in combination with $10 \%$ AGEs or $3 \mathrm{ng} / \mathrm{ml}$ LPS. As shown in figure 2, $3 \mathrm{nM}$ cortisol was significantly less able to decrease the AGE-induced IL-8 secretion compared to the LPS-induced IL-8 secretion which is highlighted in the insert of figure 2 . To distinguish the mechanism by which AGEs induce glucocorticoid resistance, the effect was modulated with compounds that interfered in different pathways involved in glucocorticoid resistance, as depicted in figure 6 . This further inquest into the underlying mechanism was only performed with cells exposed to AGEs. The cells were exposed to all three compounds in different concentrations with and without AGEs and cortisol. Adding different concentrations of rapamycin to human macrophage-like cells led to a significant improved cortisol response with approximately $10 \%$ (figure $3 \mathrm{~A}$ ). However, this improvement by rapamycin did not follow a dose-dependent pattern. Theophylline alone did not have any anti-inflammatory effect and did not improve glucocorticoid responsiveness (figure $3 \mathrm{~B}$ ). Figure $3 \mathrm{C}$ shows that the antioxidant quercetin itself at $5 \mu \mathrm{M}$ did not reduce IL-8 secretion, but when adding quercetin together with cortisol, IL-8 secretion was reduced dose-dependently and significantly. Adding $5 \mu \mathrm{M}$ quercetin by itself led a decrease of only $5 \%$ (not significant) whereas the combination of $5 \mu \mathrm{M}$ quercetin with cortisol led to a $27 \%$ reduction.

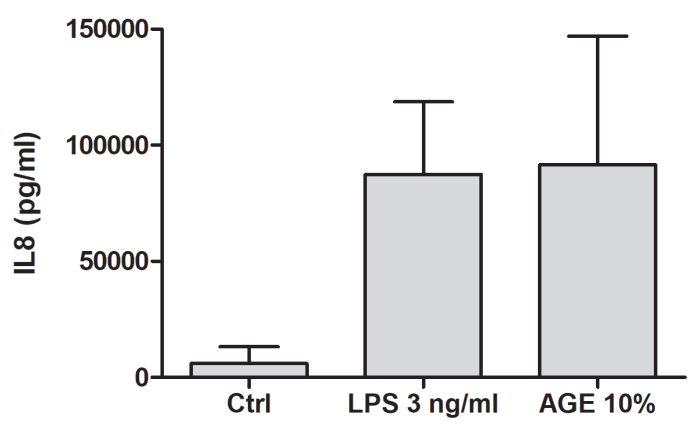

Figure 1. Absolute IL-8 secretion of human macrophage-like cells after 2 4h exposure to $3 \mathrm{ng} / \mathrm{ml}$ LPS, AGEs (10\% v/v) or control (Ctrl) condition. Data presented as mean $\pm S D, n=3, p=0.05$ (Ctrl vs LPS and Ctrl vs AGE). 


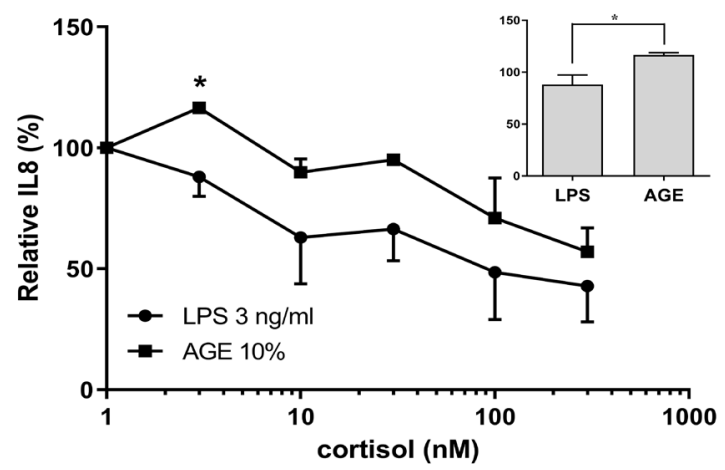

Figure 2. IL-8 secretion of human macrophage-like cells normalized to no cortisol after $24 \mathrm{~h}$ exposure to $3 \mathrm{ng} / \mathrm{ml} \mathrm{LPS}$ or AGEs $(10 \% \mathrm{v} / \mathrm{v})$ and $3-300 \mathrm{nM}$ of cortisol $(\mathrm{n}=4)$. Absolute IL-8 values of cells exposed to only LPS were $110 \pm 20$ $\mathrm{ng} / \mathrm{ml}$ and to only AGEs $154 \pm 20 \mathrm{ng} / \mathrm{ml}$,.Insert: IL-8 secretion of human macrophage-like cells relative to no cortisol after $24 \mathrm{~h}$ exposure to $3 \mathrm{ng} / \mathrm{ml}$ LPS or AGEs $(10 \% \mathrm{v} / \mathrm{v})$ in the presence of $3 \mathrm{nM}$ cortisol $(\mathrm{n}=4)$. Data presented as mean $\pm \mathrm{SD}, \mathrm{n}=4, * \mathrm{p}<0.05$ for LPS $(3 \mathrm{ng} / \mathrm{ml})$ vs AGE $(10 \%)$ in the presence of $3 \mathrm{nM}$ cortisol.

A

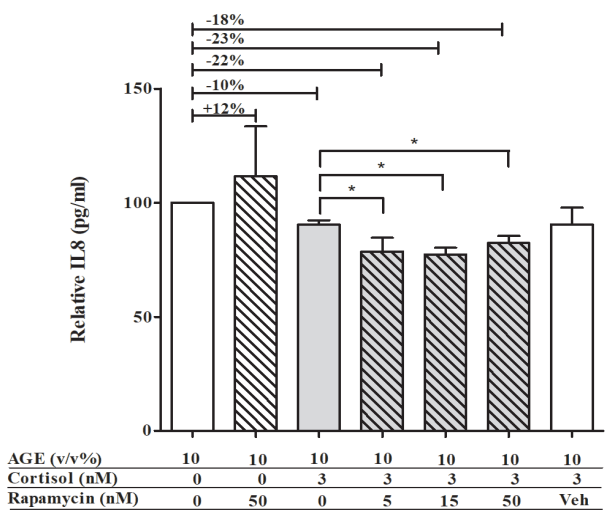

B

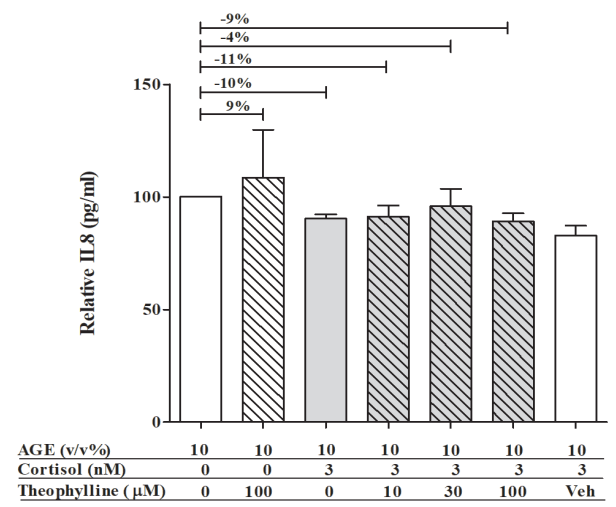

C

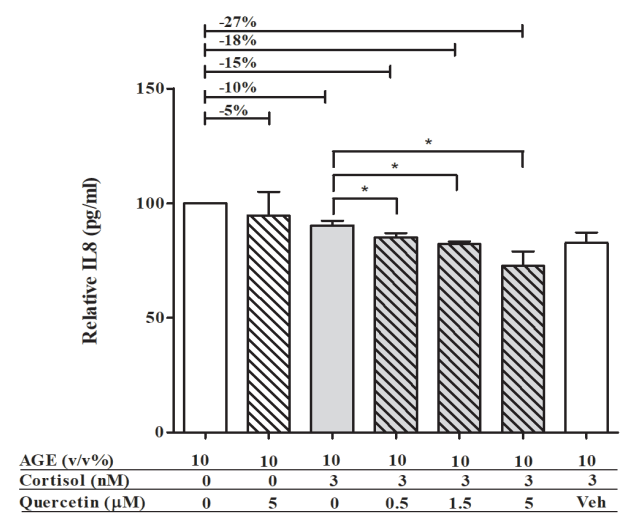

Figure 3. Relative IL-8 secretion of human macrophage-like cells after $24 \mathrm{~h}$ exposure to AGEs ( $10 \% \mathrm{~V} / \mathrm{V}), 3 \mathrm{nM}$ of cortisol and; (A) different concentrations of rapamycin; (B) different concentrations of theophylline; (C) different concentrations of quercetin. Data presented as mean $\pm S D, n=3, * p<0.05$. 


\subsection{Phosphorylation of the glucocorticoid receptor}

Activation of mTOR can lead to GR phosphorylation and since the mTOR inhibitor rapamycin was able to reduce IL-8 secretion compared to cortisol, the next step is to explore a possible role for GR phosphorylation in AGE-induced glucocorticoid resistance. The cells were exposed to either the control condition, 10\% AGEs, or $3 \mathrm{ng} / \mathrm{ml}$ LPS for $24 \mathrm{~h}$. Protein levels of GR and phosphorylated GR were assessed by Western Blot and calculated relatively to $\beta$-actin (figure 4). No significant difference was found in phosphorylation state between the different conditions.

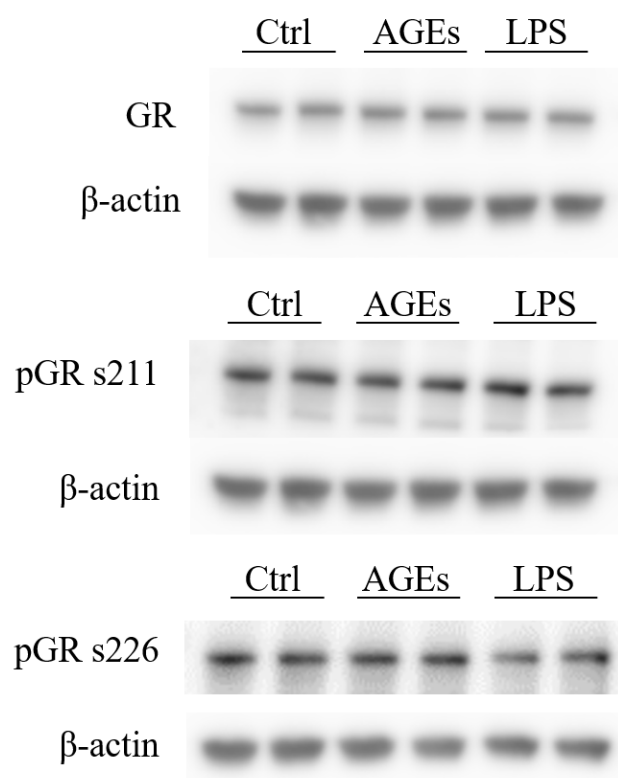

Figure 4. Protein levels of GR, phosphorylated GR at serine (s) 211, and phosphorylated GR at serine 226. $\beta$-actin is shown as loading control. 


\subsection{Intracellular ROS levels}

As shown in figure 3, quercetin dose-dependently improved glucocorticoid responsiveness. To determine whether this could be related to mitigation of intracellular ROS levels by quercetin, cells were exposed to $10 \%$ AGEs, $3 \mathrm{nM}$ cortisol, and different concentrations of quercetin for $24 \mathrm{~h}$ after which intracellular ROS levels were measured. As shown in figure 5A, 10\% AGEs increased intracellular ROS levels by approximately 50\% compared to the control condition. Cortisol ( $3 \mathrm{nM}$ ) did not reduce intracellular ROS levels, while, quercetin alone did diminish intracellular ROS levels. Figure 5B shows that combining cortisol with quercetin led to a dose-dependent reduction of intracellular ROS levels. Quercetin (5 $\mu \mathrm{M})$ significantly reduced intracellular ROS levels compared to 10\% AGEs and 10\% AGEs with cortisol.

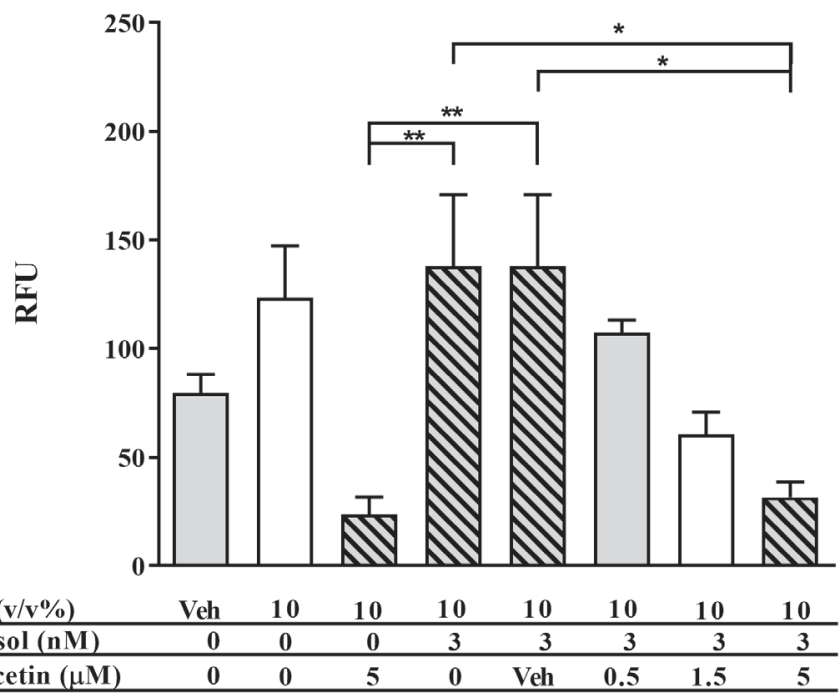

Figure 5. Intracellular ROS levels of human macrophage-like cells exposed to $10 \%$ AGEs, 3nM cortisol (C), and different concentrations of quercetin. Baseline fluorescence of THP-1 cells was set at $100 \%$. Data presented as mean $\pm S D, n=4$. 


\section{Discussion}

In the present study, it is shown for the first time that dietary AGEs induced glucocorticoid resistance of macrophage-like (THP-1) cells. Exposure of THP-1 cells to dietary AGEs increased the production of IL-8, indicating an inflammatory response, which was comparable to the pro-inflammatory effect of $3 \mathrm{ng} / \mathrm{ml}$ LPS. The glucocorticoid cortisol was able to decrease LPSinduced inflammation significantly better than AGE-induced inflammation. This indicates that exposure to dietary AGEs may lead to glucocorticoid resistance. Modulation of the pro-inflammatory response by different pharmacological compounds interfering in different steps of the anti-inflammatory mechanism of glucocorticoids showed that AGE-induced glucocorticoid resistance can be restored. The use of glucocorticoids is increasing due to the increased prevalence of chronic inflammatory diseases as asthma, rheumatoid arthritis and IBD. Despite the good effectiveness of glucocorticoids, some patients are resistant to these drugs. Glucocorticoid resistance is a disorder that is characterized by insensitivity to glucocorticoids [3]. Although much research already focused on the mechanism of glucocorticoid resistance, still it is not completely understood why some patients are insensitive to glucocorticoids, while others are not. Several mechanisms have been suggested to be involved in the development of resistance towards glucocorticoids. The present research aimed to identify the mechanism of AGE-induced glucocorticoid resistance by exposing THP1 cells to different compounds that interfere at different stages of the anti-inflammatory mechanism of glucocorticoids.

Barnes published an excellent review about the mechanisms of glucocorticoid resistance (figure 6) [3]. Phosphorylation of the GR was suggested as one important cause of such resistance. Phosphorylation of the GR at Ser226 and 211 inhibits the binding of the activated GR to GRE, resulting in a decreased expression of anti-inflammatory genes and consequently an increased expression of pro-inflammatory genes. One of the factors responsible for the phosphorylation of the GC is mTOR. Our results show that inhibition of mTOR by rapamycin increased the sensitivity of THP-1 cells to cortisol. However, no differences were found in the protein levels of phosphorylated GC (at Ser226 and 211) between control conditions and AGE-treated cells. Even though rapamycin increased the sensitivity, these results suggest that phosphorylation of the GC at the two serine residues is not the main mechanism for AGE-induced glucocorticoid resistance. Another suggested mechanism can be mediated through oxidative stress. Oxidative stressors such as ROS are able to inactivate HDAC2 through the formation of peroxynitrite and subsequent nitration of tyrosine residues of HDAC2 [16,22], thereby inducing glucocorticoid resistance [23,24]. HDAC2 normally ensures chromatin remodelling of pro-inflammatory genes, hence inactivation results in increased expression of pro-inflammatory genes, among which IL-8 [8]. It is therefore expected that antioxidants could ameliorate the sensitivity to glucocorticoids by increasing HDAC2 activity. In the present study, AGEs were found to increase the formation of ROS in THP-1 cells, which was significantly reduced by the antioxidant quercetin. Our results furthermore show that quercetin in combination with cortisol decreased IL-8 production, while quercetin alone did 
not have this effect. The inhibition of IL-8 secretion by the combination quercetin and cortisol indicate that the anti-inflammatory effect of quercetin is dependent on cortisol. This can be explained by the improvement of the cortisol response by quercetin, which is in line with previous reported data from Mitani et al. (2017) [25] that proved that quercetin was able to restore glucocorticoid resistance in U937 monocytes. Our results show that AGE-induced glucocorticoid resistance is likely to be caused by formation of ROS. A plausible explanation for this effect is the damage of HDAC2 by ROS, rendering it inactive [15,26-28]. The strong antioxidant quercetin is able to improve glucocorticoid sensitivity by neutralizing ROS. The HDAC activator theophylline did not have any effect on AGE-induced inflammation and glucocorticoid resistance. This could be due to the fact that only very low concentrations of theophylline restores HDAC activity [29]. Secondly, theophylline might in fact not act on HDAC2, as was found by Ito et al. (2002) [30]. Theophylline seems to work at HDAC1 and HDAC3 specifically, which could explain why no effect was seen in our study. However, since HDAC2 activation was not investigated in this study, future research may focus on the involvement of HDAC2 in AGE-induced glucocorticoid resistance.

Based on the results of this study and existing literature, the proposed mechanism by which AGEs induce glucocorticoid resistance is shown in figure 7. AGEs can induce ROS via two pathways. Firstly, AGEs bind to RAGE, which activates NADPH-oxidases, leading to the formation of ROS. Activation of RAGE also eventually leads to an increased iNOS expression [22,31]. AGEs themselves are formed under oxidative conditions and are pro-oxidant molecules which are also able to form ROS $[32,33]$. This AGE-induced ROS formation then inactivates HDAC2, leading to glucocorticoid resistance. What is the implication of these findings for humans? It is under debate whether dietary AGEs are absorbed within the human body, but they are present within the gastro-intestinal tract. Additionally, glucocorticoid resistance occurs in approximately $20 \%$ of people with IBD [1,2]. The results of the current study show that the type of food IBD patients eat may be of large importance to IBD patients suffering from glucocorticoid resistance. Studies are currently underway in our lab to analyze the digestion of dietary AGEs to investigate the effects of dietary AGEs on inflammation. In this way we hope to get a better picture of the role of this studies' phenomena on the in vivo situation. Next to the potential negative effect of a high-AGE diet on glucocorticoid sensitivity, our study shows that at least one specific polyphenol, quercetin can increase glucocorticoid responsiveness. Additionally, another polyphenol, curcumin, has been shown to have a similar effect in a COPD model [34]. People eating high-AGE diets, containing many fried, and baked foods, might also eat less fruits and vegetables that contain many of the polyphenols in our diet. The question whether an inverse relation exists between AGE and polyphenol intake can be studied in the future. 


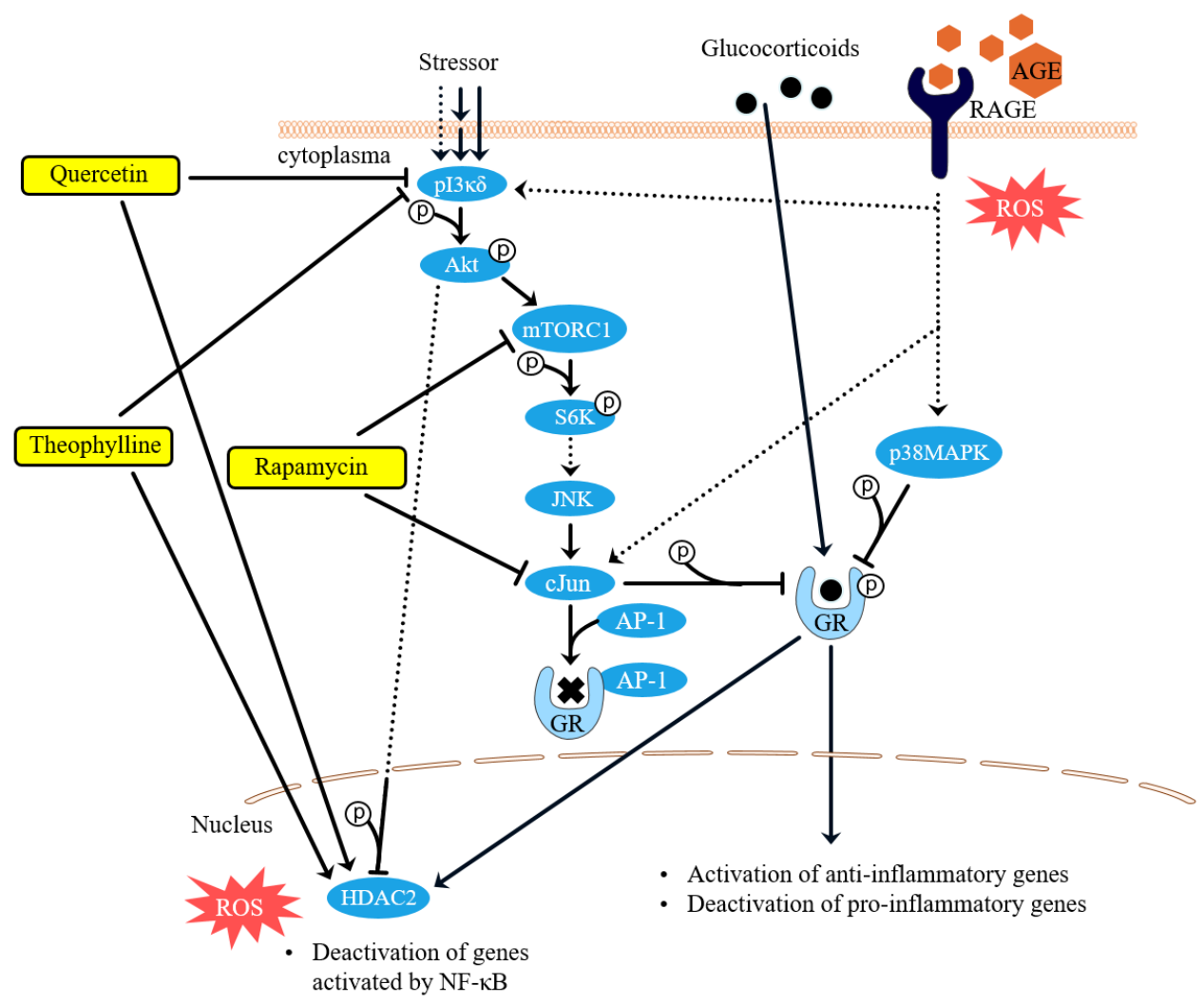

Figure 6. Mechanism of glucocorticoid resistance and the addition of the receptor for advanced glycation endproducts (RAGE) $[3,19]$. Glucocorticoids diffuse through the cellmembrane into the cell where they bind to the glucocorticoid receptor (GR). This complex then translocates into the nucleus where it exerts its effect. Under glucocorticoid resistance-conditions, a stressor activates phosphoinositide 3-kinase delta (pI3K $\delta$ ), thereby activating the PI3K/AKT/mTOR pathway leading to an increase in cJun protein which phosphorylates the GR and induces binding of the AP-1 protein to the GR, inhibiting the glucocorticoid-GR complex from translocating to the nucleus. We suggest that AGEs bind to RAGE thus activating both, PI3K- and p38 mitogen-activated protein kinase (p38MAPK)mediated phosphorylation of the GR. On the left side, the proposed effects of different compounds (in yellow) on the glucocorticoid resistance are shown. Quercetin inhibits PI3K and protects histone deacetylase 2 (HDAC2). Rapamycin is an mTOR and cJun inhibitor. Theophylline inhibits PI3K and activates HDAC. 


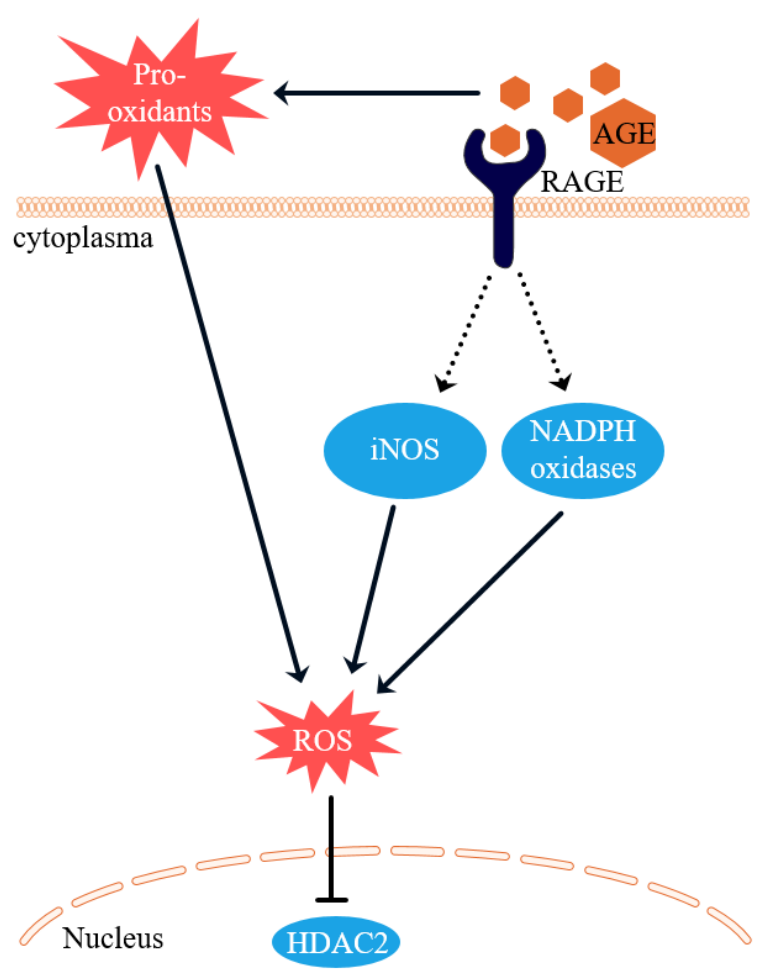

Figure 7. Proposed mechanism of glucocorticoid resistance caused by AGEs. The pro-oxidative AGEs lead to ROS formation. AGEs bind to RAGE, RAGE activates NADPH oxidases and upregulates iNOS which in turn induces ROS. HDAC2 is inhibited by ROS.

\section{Conclusions}

In conclusion: The present study showed for the first time indications that inflammation induced by AGEs is less well mitigated by cortisol than LPS-induced inflammation. Indicating that AGEs may cause glucocorticoid resistance by increasing intracellular ROS. Quercetin is able to improve the glucocorticoid response by reducing intracellular ROS levels. 


\section{References}

1. Tung, J.; Loftus, E.V., Jr.; Freese, D.K.; El-Youssef, M.; Zinsmeister, A.R.; Melton, L.J., 3rd; Harmsen, W.S.; Sandborn, W.J.; Faubion, W.A., Jr. A population-based study of the frequency of corticosteroid resistance and dependence in pediatric patients with Crohn's disease and ulcerative colitis. Inflamm Bowel Dis 2006, 12, 1093-1100, doi:10.1097/01.mib.0000235835.32176.85.

2. Munkholm, P.; Langholz, E.; Davidsen, M.; Binder, V. Frequency of glucocorticoid resistance and dependency in Crohn's disease. Gut 1994, 35, 360-362.

3. Barnes, P.J. Mechanisms and resistance in glucocorticoid control of inflammation. J Steroid Biochem Mol Biol 2010, 120, 76-85, doi:10.1016/j.jsbmb.2010.02.018.

4. Rhen, T.; Cidlowski, J.A. Antiinflammatory action of glucocorticoids--new mechanisms for old drugs. N Engl J Med 2005, 353, 1711-1723, doi:10.1056/NEJMra050541.

5. Ito, K.; Barnes, P.J.; Adcock, I.M. Glucocorticoid receptor recruitment of histone deacetylase 2 inhibits interleukin-1beta-induced histone $\mathrm{H} 4$ acetylation on lysines 8 and 12. Mol Cell Biol 2000, 20, 6891-6903.

6. Clark, A.R. MAP kinase phosphatase 1: a novel mediator of biological effects of glucocorticoids? J Endocrinol 2003, 178, 5-12.

7. Barnes, P.J. Corticosteroid effects on cell signalling. Eur Respir J 2006, 27, 413-426, doi:10.11 83/09031936.06.00125404.

8. Ito, K.; Yamamura, S.; Essilfie-Quaye, S.; Cosio, B.; Ito, M.; Barnes, P.J.; Adcock, I.M. Histone deacetylase 2-mediated deacetylation of the glucocorticoid receptor enables NF-kappaB suppression. J Exp Med 2006, 203, 7-13, doi:10.1084/jem.20050466.

9. Bergmann, M.W.; Staples, K.J.; Smith, S.J.; Barnes, P.J.; Newton, R. Glucocorticoid inhibition of granulocyte macrophage-colony-stimulating factor from T cells is independent of control by nuclear factor-kappaB and conserved lymphokine element 0. Am J Respir Cell Mol Biol 2004, 30, 555-563, doi:10.1165/rcmb.2003-02950C.

10. Weigel, N.L.; Moore, N.L. Steroid receptor phosphorylation: a key modulator of multiple receptor functions. Mol Endocrinol 2007, 21, 2311-2319, doi:10.1210/me.2007-0101.

11. Matthews, J.G.; Ito, K.; Barnes, P.J.; Adcock, I.M. Defective glucocorticoid receptor nuclear translocation and altered histone acetylation patterns in glucocorticoid-resistant patients. J Allergy Clin Immunol 2004, 113, 1100-1108, doi:10.1016/j.jaci.2004.03.018.

12. Szatmary, Z.; Garabedian, M.J.; Vilcek, J. Inhibition of glucocorticoid receptor-mediated transcriptional activation by p38 mitogen-activated protein (MAP) kinase. The Journal of biological chemistry 2004, 279, 43708-43715, doi:10.1074/jbc.M406568200.

13. Miller, A.L.; Webb, M.S.; Copik, A.J.; Wang, Y.; Johnson, B.H.; Kumar, R.; Thompson, E.B. p38 Mitogen-activated protein kinase (MAPK) is a key mediator in glucocorticoidinduced apoptosis of lymphoid cells: correlation between p38 MAPK activation and sitespecific phosphorylation of the human glucocorticoid receptor at serine 211 . Mol Endocrinol 2005, 19, 1569-1583, doi:10.1210/me.2004-0528.

14. Irusen, E.; Matthews, J.G.; Takahashi, A.; Barnes, P.J.; Chung, K.F.; Adcock, I.M. p38 Mitogenactivated protein kinase-induced glucocorticoid receptor phosphorylation reduces its activity: role in steroid-insensitive asthma. J Allergy Clin Immunol 2002, 109, 649-657.

15. Ito, K.; Hanazawa, T.; Tomita, K.; Barnes, P.J.; Adcock, I.M. Oxidative stress reduces histone deacetylase 2 activity and enhances IL-8 gene expression: role of tyrosine nitration. Biochem Biophys Res Commun 2004, 315, 240-245, doi:10.1016/j.bbrc.2004.01.046.

16. Kirkham, P.; Rahman, I. Oxidative stress in asthma and COPD: antioxidants as a therapeutic strategy. Pharmacol Ther 2006, 111, 476-494, doi:10.1016/j.pharmthera.2005.10.015.

17. Ruijters, E.J.; Haenen, G.R.; Willemsen, M.; Weseler, A.R.; Bast, A. Food-Derived Bioactives Can Protect the Anti-Inflammatory Activity of Cortisol with Antioxidant-Dependent and -Independent Mechanisms. Int J Mol Sci 2016, 17, 239, doi:10.3390/ijms17020239.

18. van der Lugt, T.; Weseler, A.; Gebbink, W.; Vrolijk, M.; Opperhuizen, A.; Bast, A. Dietary Advanced Glycation Endproducts Induce an Inflammatory Response in Human Macrophages in Vitro. Nutrients 2018, 10, 1868.

19. Xie, J.; Mendez, J.D.; Mendez-Valenzuela, V.; Aguilar-Hernandez, M.M. Cellular signalling of the receptor for advanced glycation end products (RAGE). Cellular signalling 2013, 25, 
2185-2197, doi:10.1016/j.cellsig.2013.06.013.

20. Poulsen, M.W.; Hedegaard, R.V.; Andersen, J.M.; de Courten, B.; Bugel, S.; Nielsen, J.; Skibsted, L.H.; Dragsted, L.O. Advanced glycation endproducts in food and their effects on health. Food and chemical toxicology : an international journal published for the British Industrial Biological Research Association 2013, 60, 10-37, doi:10.1016/j. fct.2013.06.052.

21. Ruiz-Leal, M.; George, S. An in vitro procedure for evaluation of early stage oxidative stress in an established fish cell line applied to investigation of PHAH and pesticide toxicity. Mar Environ Res 2004, 58, 631-635, doi:10.1016/j.marenvres.2004.03.054.

22. Bansal, S.; Siddarth, M.; Chawla, D.; Banerjee, B.D.; Madhu, S.V.; Tripathi, A.K. Advanced glycation end products enhance reactive oxygen and nitrogen species generation in neutrophils in vitro. Mol Cell Biochem 2012, 361, 289-296, doi:10.1007/s11010-0111114-9.

23. Barnes, P.J.; Adcock, I.M. Glucocorticoid resistance in inflammatory diseases. Lancet 2009, 373, 1905-1917, doi:10.1016/S0140-6736(09)60326-3.

24. Ruijters, E.J.; Haenen, G.R.; Weseler, A.R.; Bast, A. The cocoa flavanol (-)-epicatechin protects the cortisol response. Pharmacol Res 2014, 79, 28-33, doi:10.1016/j. phrs.2013.11.004.

25. Mitani, A.; Azam, A.; Vuppusetty, C.; Ito, K.; Mercado, N.; Barnes, P.J. Quercetin restores corticosteroid sensitivity in cells from patients with chronic obstructive pulmonary disease. Exp Lung Res 2017, 43, 417-425, doi:10.1080/01902148.2017.1393707.

26. Barnes, P.J. Role of HDAC2 in the pathophysiology of COPD. Annu Rev Physiol 2009, 71, 451 464, doi:10.1146/annurev.physiol.010908.163257.

27. To, Y.; Ito, K.; Kizawa, Y.; Failla, M.; Ito, M.; Kusama, T.; Elliott, W.M.; Hogg, J.C.; Adcock, I.M.; Barnes, P.J. Targeting phosphoinositide-3-kinase-delta with theophylline reverses corticosteroid insensitivity in chronic obstructive pulmonary disease. Am J Respir Crit Care Med 2010, 182, 897-904, doi:10.1164/rccm.200906-09370C.

28. Randall, M.J.; Haenen, G.R.; Bouwman, F.G.; van der Vliet, A.; Bast, A. The tobacco smoke component acrolein induces glucocorticoid resistant gene expression via inhibition of histone deacetylase. Toxicol Lett 2016, 240, 43-49, doi:10.1016/j. toxlet.2015.10.009.

29. Marwick, J.A.; Wallis, G.; Meja, K.; Kuster, B.; Bouwmeester, T.; Chakravarty, P.; Fletcher, D.; Whittaker, P.A.; Barnes, P.J.; Ito, K., et al. Oxidative stress modulates theophylline effects on steroid responsiveness. Biochem Biophys Res Commun 2008, 377, 797802, doi:10.1016/j.bbrc.2008.10.065.

30. Ito, K.; Lim, S.; Caramori, G.; Cosio, B.; Chung, K.F.; Adcock, I.M.; Barnes, P.J. A molecular mechanism of action of theophylline: Induction of histone deacetylase activity to decrease inflammatory gene expression. Proc Natl Acad Sci U S A 2002, 99, 8921-8926, doi:10.1073/pnas.132556899.

31. Wautier, M.P.; Chappey, O.; Corda, S.; Stern, D.M.; Schmidt, A.M.; Wautier, J.L. Activation of NADPH oxidase by AGE links oxidant stress to altered gene expression via RAGE. Am J Physiol Endocrinol Metab 2001, 280, E685-694, doi:10.1152/ ajpendo.2001.280.5.E685.

32. Rowan, S.; Bejarano, E.; Taylor, A. Mechanistic targeting of advanced glycation end-products in age-related diseases. Biochim Biophys Acta Mol Basis Dis 2018, 1864, 3631-3643, doi:10.1016/j.bbadis.2018.08.036.

33. Nowotny, K.; Jung, T.; Hohn, A.; Weber, D.; Grune, T. Advanced glycation end products and oxidative stress in type 2 diabetes mellitus. Biomolecules 2015, 5, 194-222, doi:10.3390/biom5010194.

34. Meja, K.K.; Rajendrasozhan, S.; Adenuga, D.; Biswas, S.K.; Sundar, I.K.; Spooner, G.; Marwick, J.A.; Chakravarty, P.; Fletcher, D.; Whittaker, P., et al. Curcumin restores corticosteroid function in monocytes exposed to oxidants by maintaining HDAC2. Am J Respir Cell Mol Biol 2008, 39, 312-323, doi:10.1165/rcmb.2008-00120C. 

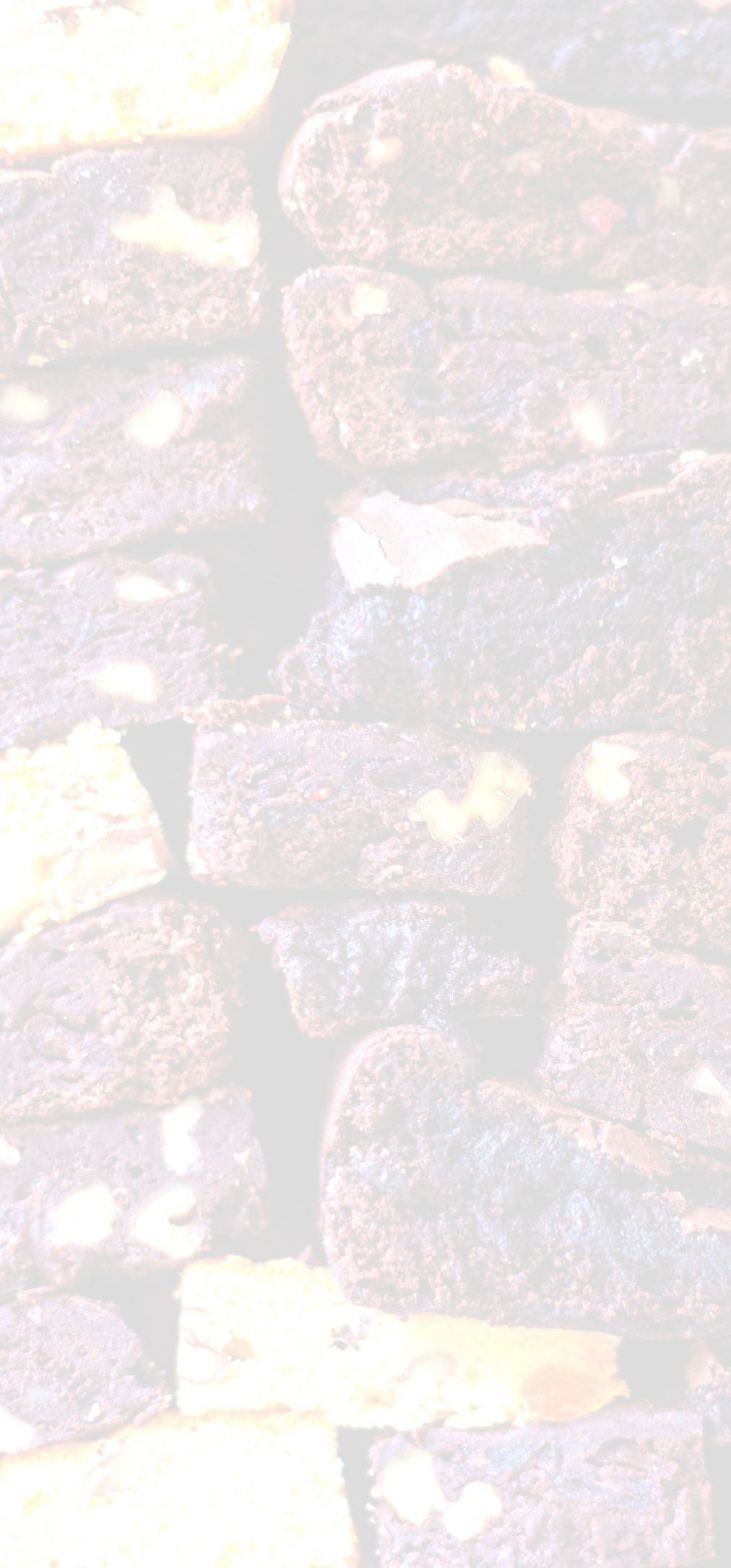


\section{Chapter 6}

Summary and discussion 


\section{Main findings}

dAGEs induce pro-inflammatory effects in macrophages and have implications for human digestion, the cellular effects in gastrointestinal cells are thermal treatment dependent.

During food processing, food products usually undergo thermal treatment. Under the influence of high temperatures, dietary advanced glycation endproducts (dAGEs) are formed via the Maillard reaction. Endogenous advanced glycation endproducts (AGEs) are known for their pro-inflammatory effects. However, the effects of dAGEs are less well-known, especially the effects in the gastrointestinal (GI) tract. The aim of this thesis was to investigate the potential local effects of dAGEs. To this end, 1) the pro-inflammatory potential of dAGEs was measured through TNF- $\alpha$ secretion, 2) the digestion of dAGEs was assessed using the sophisticated TNO gastrolntestinal Model (TIM-1), 3) the pro-inflammatory potential of digested dAGEs was assessed and the effects of dAGEs on gastrointestinal cells, and 4) the glucocorticoid sensitivity of cells exposed to dAGEs was assessed to see in what way dAGEinduced inflammation may be mitigated.

In chapter 2 we examined the TNF- $\alpha$ secretion and cell viability of human macrophage-like cells exposed to glycated casein (GC) and the individual (free) dAGEs Ne-carboxymethyllysine $(\mathrm{CML}), \mathrm{N} \varepsilon$-carboxyethyllysine (CEL), and methylglyoxal-derived hydroimidazolone 1 (MG-H1). GC, which includes protein-bound dAGEs, induced TNF- $\alpha$ secretion in a dose dependent fashion, whereas the free dAGEs did not. Long heating times of GC reduced cell viability. Blocking the receptor for advanced glycation endproducts (RAGE) significantly reduced TNF- $\alpha$ secretion. The dAGE-protein binding stays intact during in vitro digestion of GC, ginger biscuits and apple juice (chapter 3). Glycation of casein slows down casein digestion compared to unglycated casein (UC). During GI digestion, endogenous formation of AGEs occurs largely depending on the type of food product. Exposing the human macrophage-like cells to the digested dAGEs induced a higher TNF- $\alpha$ secretion than undigested dAGEs (chapter 4). The largest molecules in the GC show the highest pro-inflammatory potential, while the smallest fractions show no pro-inflammatory effect. In Gl epithelial cells long heating times decreased cell viability while any heat treatment led to a decrease in intracellular reactive oxygen species (ROS) compared to the unheated samples. This indicates the importance of thermal treatment on the cellular effects. Inflammatory bowel disease (IBD) patients suffer from inflammation in the Gl tract and one of the most commonly used anti-inflammatory drugs are glucocorticoids. dAGE-induced inflammation decreased glucocorticoid sensitivity of human macrophage-like cells (chapter 5). This dAGE-induced glucocorticoid resistance was mitigated by supplementation of the polyphenol quercetin, which can possibly be explained through scavenging of intracellular ROS. 


\section{Discussion}

\section{dAGEs and pro-inflammatory effects: size matters}

One of the reasons why the pro-inflammatory effect of dAGEs has been disputed in recent years is because the evidence on pro-inflammatory effects of dAGEs has not been consistent. Some studies found pro-inflammatory effects, others did not. Buetler et al. (2011) attributed the conflicting results to endotoxin presence, since they found no TNF- $\alpha$ secretion after eliminating their endogenous AGEs by TF-114 extraction. Because of this, in chapter 2 we first tested our dAGEs on endotoxin presence and only used endotoxin-free samples to treat the cells. Our dAGEs did induce TNF- $\alpha$ secretion, which could be attenuated by blocking RAGE with an antagonist. Opposed to the effect of GC, individual dAGEs CML, CEL, MG-H1, and acrylamide did not induce any TNF- $\alpha$ secretion. This indicates the importance of protein binding in order to exert a pro-inflammatory effect. This hypothesis has been mentioned in multiple studies $[1,2]$. The importance of dAGE-protein binding might be related to their ability to bind RAGE. Which specific AGEs bind to RAGE is under much debate. Free AGEs such as CEL and CML do not seem to bind to RAGE, whereas AGE-modified proteins and AGEs containing a peptide backbone do [1-5]. This suggests a possible size-dependent interaction between RAGE and its ligands, which might be due to oligomerization of RAGE in the cell membrane. Therefore, protein-bound AGEs are able to bind more strongly to oligomerized RAGE compared to individual RAGE [3]. Xue et al. (2014) found indications that in the case of MG-H1-modified peptides, the imidazolone ring of MG-H1 seems to be important in its binding to RAGE [5].

The large structural differences between the RAGE ligands are striking and make it complicated to find the exact elements necessary for ligand binding to RAGE. To explore the size-dependent effects of dAGEs, in chapter 4 GC was fractionated according to size and the different fractions exposed to human macrophage-like cells. Our results showed that especially large fractions induced high levels of TNF- $\alpha$ secretion. Strikingly, individual fractionated samples led to a higher TNF- $\alpha$ secretion than the unfractionated samples. This indicates that different sizes of dAGEs can have different biological effects. Different sub-sets of molecules are formed during the Maillard reaction, called Maillard reaction products (MRPs). A sub-set named early glycation products have been shown in one study to exert anti-inflammatory effects by polarizing macrophages to the more anti-inflammatory M2 type in C57BL/6 male mice [6]. In a study of Oh et al. (2017), lysine-galactose MRPs of 3 to $10 \mathrm{kDa}$ reduced LPS-induced inflammation in RAW264.7 cells [7]. This evidence of both pro-inflammatory and antiinflammatory properties of MRPs might explain why the unfractionated GC induces less TNF- $\alpha$ secretion than the individual fractions; the smaller fraction may possess anti-inflammatory properties, through potential inhibition of the translocation of NF-KB subunits, that level out the pro-inflammatory effects [8]. Despite the potential anti-inflammatory effects of smaller MRPs, the results in chapter 4 show that, at least in our food-based matrix, the proinflammatory effects predominate the anti-inflammatory properties of smaller MRPs. 


\section{Digestion of dAGEs}

Before dAGEs may potentially exert these pro-inflammatory effects locally in the intestines or even systemically, they will first need to undergo digestion. Studies into the effect of gastric digestion on dAGEs are scarce and most studies are conducted using static in vitro models. In these models food products are most commonly exposed to synthetic gastric juice and pepsin set at a pH value of $2-2.5$ for two hours. Human gastric digestion however is not a static environment; it is dynamic and includes peristaltic mixing and alternating pH levels [9]. The physiological pH levels in the stomach differ between $1-1.5$ for basal levels and 5 - 7 immediately after ingestion. After approximately three hours of ingestion, the $\mathrm{pH}$ returns to basal levels [10]. Additionally, digestive fluids and meal components are removed from the GI tract by both passive and active absorption [16]. Unfortunately, the static in vitro models do not take such physiological parameters into account.

Since almost all available studies on the digestion of dAGEs used these physiologically less relevant static in vitro models, in chapter 3 we assessed the digestion of dAGEs and their protein binding in the sophisticated TNO gastrolntestinal Model (TIM-1). TIM-1 is a dynamic in vitro model which mimics the upper human $\mathrm{Gl}$ tract. This model takes multiple parameters into account, such as: dynamic $\mathrm{pH}$ curves, peristaltic mixing, addition of bile and pancreatic digestive enzymes, and passive absorption of fluids and food compounds. We analysed both our food-based matrix (GC) and two actual food products: ginger biscuits and apple juice. Our results showed that the protein binding of dAGEs is able to survive gastric and small intestinal digestion and consequently stays intact throughout the whole $\mathrm{Gl}$ tract. We also found a hampered digestion of GC compared to UC.

The results of our study are underlined by different studies using static in vitro models. In order to determine the effect of gastric digestion on dAGEs, Hellwig et al. (2013) exposed casein modified with $\mathrm{N} \varepsilon$-fructoselysine, $\mathrm{CML}$, and lysinoalanine to synthetic gastric juice and pepsin with a $\mathrm{pH}$ value of 2 at $37^{\circ} \mathrm{C}$ for two hours. In this study, modifying casein lead to a reduction of digestibility of casein in the intestines [11]. Although most of the physical parameters were taken into account, all dAGEs were exposed to the gastric juices for the complete two hours. This is not the case in the human body where constantly a small portion of the meal is transported to the duodenum and gastric emptying time is depending on the type of food [12, 13]. In addition, several other studies have been published on the digestibility of dAGEs in the small and large intestine. Joubran et al. (2015) assessed the digestive proteolysis of glycated $\alpha$-lactalbumin in adults and infant gastro-duodenal conditions [14]. The type of carbohydrate used in the experiments determined the extent to which proteolysis could occur. Digestion of glycated $\alpha$-lactalbumin led to higher anti-oxidative properties, while other oligosaccharides protected against proteolysis. However, the details on what gastric conditions were used, were not disclosed in the paper and their method of thermal processing corresponded to neither endogenous nor exogenous conditions. Underlining these results, Zhao et al. (2017) saw a decrease in digestibility of $\beta$-casein and $\beta$-lactoglobulin after glycation with glyoxal [15]. In a different study, Pinto et al. (2013) subjected $\beta$-lactoglobulin and $\beta$-casein to a heating 
regime of $90^{\circ} \mathrm{C}$ for 24 hours and found larger aggregates of casein combined with glucose than native casein, while glycation retarded the aggregation of $\beta$-lactoglobulin. Also, when heated both glucose-protein combinations were less sensitive to enzymatic digestion than their heated native counterparts [16]. Moscovici et al. (2014) found a decreased sensitivity of bovine lactoferrin glycated with fructo-oligosaccharides to proteolysis after heating at $60^{\circ} \mathrm{C}$ in a water restricted environment for 12 hours, and an enhanced proteolysis after 24 hours of heating [17]. Both studies indicate the importance of the starting product and thermal treatment on the effect on tertiary structure of the protein and thereby its digestibility.

In addition to the above mentioned experimental studies, an excellent systematic review by van Lieshout et al. (2019) analysed the effects of glycation on protein digestibility and concluded that glycation decreases protein digestibility [18]. One explanation for the reduction of protein digestibility by the MR lies in the nature of the reaction itself. The major reactive site for the $M R$ is the $\varepsilon$-amino group of lysine, and to a lesser extent the guanido group of arginine [19]. These same residues are also tryptic binding sites [20]. Consequently, this leads to a direct hindrance of the proteolytic cleavage sites [20,21]. Additionally, indirect steric hindrance of the proteolytic cleavage sites can occur through binding to residues close to proteolytic cleavage sites, or it can cause cross-linking that inhibits access for proteases [18]. Alternatively, glycation may also promote proteolysis by opening up the tertiary structure of proteins (conjugation may interfere with refolding the protein after heating [21]), which was observed in the study by Moscovici et al. [17]. A recent human trial administering milk protein to healthy young men, which was glycated to different extents, found a reduced Iysine availability that was dependent on the level of glycation. This reduced availability was only seen for lysine, not for the other amino acids [22]. Additionally, Joubran et al. (2017) found that the MR changes the functionality of the whey protein $\alpha$-lactalbumin. They also found that the specific carbohydrate involved in the reaction determined the change of the protein [21].

Based on these studies it can be concluded that dAGEs themselves, and their protein binding, are not broken down in GI tract. The extent of digestion of dAGEs is depending on the raw materials and thermal treatment. Additionally, the binding of dAGEs to proteins leads to a decreased digestibility and bioavailability of amino acids, with lysine in particular, leading to a loss of essential and nonessential amino acids in food products.

\section{Formation of AGEs in the GI tract}

In addition to not being hydrolysed in the GI tract, the results of our TIM-1 study in chapter 3 show a large increase in AGEs formed in the GI tract during digestion in the small intestinal tract. This effect was especially large in the digests of ginger biscuits, for which an increase of up to $400 \%$ of protein-bound MG-H1 was detected. These results correspond to previous studies using different static in vitro studies, which have also showed endogenous formation of AGEs within the GI tract. One of these studies showed that simulated digestion of ovalbumine with fructose alone produced compounds with a fluorescence spectrum that 
is characteristic for AGEs [23]. In line with this, DeChristopher suggested that the formation of fructose-derived AGEs in the small intestine can be explained by fructose malabsorption. This may consequently lead to increased serum AGE levels [24, 25]. Fructose malabsorption can occur when the ratio of fructose to glucose is higher than 1:1, or when an individual has a fructose transporter deficiency. Ratios higher than one can be found in for instance high fructose corn syrup (HFCS) and apple juice. Normally, fructose is passively transported in the intestinal cell by glucose transporter 5 (GLUT5) and transported into the bloodstream by glucose transporter 2 (GLUT2) [26]. However, evidence suggests that glucose enhances fructose absorption in humans possibly through co-absorption with glucose via GLUT2 [27, 28]. As a consequence of fructose malabsorption, its levels will increase inside the intestines, which might eventually lead to the increased formation of AGEs due to the more reactive nature of fructose in the MR than glucose. Although fructose-specific AGEs are not well defined, fructose seems to be 7- 10 times more sensitive to glycation than glucose [23, 29]. However, many of these studies focussed on fructose based dAGEs. The effect of glucose on the intestinal AGE formation was also shown by Martinez-Saez in an unheated meal resembling system [30]. Helou et al. (2017) proposed a different hypothesis for the formation of AGEs in the GI tract. In their study they used the TIM-1 model to assess the digestion of fluorescent soluble melanoidins/dAGEs from bread crust, and found an increase in fluorescent molecules in the digests [31]. The authors concluded that, since the TIM-1 model only has a temperature of $37^{\circ} \mathrm{C}$, the increase was related to the release of soluble melanoidins from larger insoluble melanoidin-skeletons. This would mean that proteins conceal glycated parts that are released during glycation. However, our study included a hydrolysation step in which the dAGEs were detached from their proteins, circumventing this problem. We show that new AGEs are being formed and not released from their structures. UC did not lead to any endogenous formation of AGEs, indicating that the thermal treatment that food products undergo is vital for the formation of endogenous AGEs. In addition, in our study AGEs were only formed endogenously after digestion of products that also contained AGEs, which might indicate an underlying need for the presence of dAGE precursors in food, such as glyoxal and methylglyoxal. To our knowledge, no studies have focused on the role of dAGE precursors on intestinal AGE formation and this might be an interesting topic for future research.

\section{Effects of dAGEs in the GI tract}

After the ingestion of thermally processed food products, dAGEs enter the human GI tract. Evidence on the local effects of dAGEs in the GI tract remain scarce. An important question that arises is what the influence of the $\mathrm{GI}$ passage is on the pro-inflammatory potential dAGEs. To investigate this, the GC digests from chapter 3 were assessed on endotoxin presence and endotoxin-free samples were used to expose human macrophage-like cells in chapter 4. Our results showed that digested dAGEs maintain their high pro-inflammatory potential as illustrated by the increasing trend on the TNF- $\alpha$ secretion compared to undigested dAGEs. After correction for protein content, digested dAGEs are approximately four times more 
pro-inflammatory than undigested dAGEs. It is important to note that the concentrations of digested GC used in this study are 100X smaller than the concentrations that are encountered in vivo in the human GI tract. In order to determine the effects of digested dAGEs on GI epithelial cells (Caco-2), similar experiments were conducted with these cells. Unfortunately, the Caco- 2 cells did not respond well to the enzymes and buffers from the TIM-1 model, so undigested GC was used for further assessment of the effect of dAGEs on Caco-2 cells. The effect of dAGEs on the cell viability of Caco-2 cells was dependent on the preparation time. Cell viability decreased significantly when cells were exposed to GC heated for 120 minutes, whereas the 15 minutes heated GC barely influenced cell viability. Unfortunately, we were not able to assess cytokine secretion of Caco-2 cells. Intracellular reactive oxygen species (ROS) production of Caco-2 cells decreased after exposure to heated GC, indicating an antioxidative effect. Remarkably, UC did increase intracellular ROS. This is opposite to the effects seen in chapter 5, where GC caused an increase in intracellular ROS in human macrophagelike cells. Hence, the biological effects of dAGEs seem to be both thermal treatment and cell-type dependent.

The thermal treatment dependency might be attributed to the different MRPs formed during food processing. The molecules formed at the last stage of the MR are melanoidins [32]. Melanoidins are chemically not well-defined, but literature suggests that melanoidins have anti-oxidative properties, which might be one explanation for the reduction seen in intracellular ROS production after heating of GC in our study. Yáñez et al. (2018) analysed the effect of the rate of MR on the formation of antioxidants during the reaction, through the measurement of DPPH radical scavenging capacity of MRPs. Their results showed formation of antioxidants especially in the intermediate and final stage [33]. Additionally, a faster $M R$ rate (in the presence of glucose) led to a lower anti-oxidative capacity than when the MR progressed at a slow rate (in the presence of lactose). An explanation for the higher antioxidant formation can be the difference in chemical structure between lactose and glucose, leading to the formation of different compounds through the MR. In a different study aimed to analyse the anti-oxidative capacity of MRPs, biscuits were heated for different amounts of time, after which they were digested using a static in vitro digestive model [80]. Subsequently the digests were incubated with human faeces, after which DPPH, FRAP, and ICRED were measured. The results show increased anti-oxidative capacity after digestion and fermentation with faeces at higher preperation temperatures and longer durations. The following in vivo study in which WT male Wistar rats were given diets differing in MRP content showed possible probiotic activity in the colon for the diets high in MRPs [34].

One of the major whey proteins in milk and infant formula is $\alpha$-lactalbumin. This protein possesses several biological properties among which serving as an antioxidant. Joubran et al. (2017) assessed the effect of glycation of $\alpha$-lactalbumin with several carbohydrates (glucose, galactose and galacto-oligosaccharides) on its anti-oxidative properties and saw an increase in anti-oxidative capacity of glycated $\alpha$-lactalbumin after in vitro digestion [21]. Chuyen et al. (1998) attempted to measure the anti-oxidative capacity of specifically Amadori products and 
melanoidins and observed anti-oxidative properties by both compound groups. With higher capacity when the molecular structures of the melanoidins were larger [35]. Opposite to the large melanoidin polymers, small MRPs have been shown to possess both anti-oxidative and pro-oxidative properties, depending on the amino acid and sugar starting the reaction. Oh et al. (2017) stimulated RAW264.7 macrophages with LPS together with different sugar-amino acid MRPs [7]. Lysine derived MRPs reduced LPS induced nitric oxide (NO) production, while arginine derived MRPs increased NO production. Especially fractions containing molecule sizes between 1 and 10 kDa of lysine derived MRPs reduced LPS-induced NO production.

In addition to the inherent anti-oxidative properties of MRPs, bifidobacteria have been shown to be capable of using bread melanoidins as a carbon source, indicating a prebiotic potential of bread melanoidins. However, the melanoidins were not digested in a digestion model prior to microbial exposure [36]. Next to fermentation of dAGEs by the microbiota, due to the decreased digestibility of glycated proteins, unabsorbed amino acids and proteins are also transported into the colon. Protein fermentation in the colon is often detrimental due to the formation of several products that negatively affect the colon such as ammonia, amines, phenols and sulfides [37, 38]. As a consequence of dAGE fermentation by the microbiota and the accompanying produced compounds, the composition of the microbiota and its production of short-chain fatty acids (SCFA), which are generally considered antiinflammatory, can change $[39,40]$.

Our results have shown positive health effects of MRPs on GI cells and negative health effects on macrophages. This gives the indication that dAGEs may not cause inflammation in the GI tract of healthy people. However, people who already have a large presence of macrophages in their GI tract, such as IBD patients, may experience negative effects from dAGEs. Proteinbound dAGEs may aggravate the already existing inflammation. The most commonly used antiinflammatory drugs used in IBD patients are glucocorticoids. Despite the good effectiveness of glucocorticoids, some patients are resistant to these drugs. Glucocorticoid resistance is a disorder that is characterized by insensitivity to glucocorticoids [41]. Approximately $20 \%$ of IBD patients suffer from glucocorticoid resistance $[42,43]$. In chapter 5 , we investigated whether the dAGE-induced IL-8 secretion from macrophages can be mitigated by glucocorticoids. The efficiency of the endogenous form of glucocorticoids, cortisol, was tested on both LPSinduced inflammation and dAGE-induced inflammation. Our results showed that cortisol was less able to reduce dAGE-induced inflammation, indicating that exposure to dAGEs may lead to glucocorticoid resistance. The polyphenol quercetin was able to at least partly reverse the dAGE-induced glucocorticoid resistance by reducing intracellular ROS in human macrophagelike cells. This indicates that next to the potential to aggravate intestinal inflammation, dAGEs from food products may interfere with the effectiveness of glucocorticoids, for instance in IBD patients, which are aimed to reduce inflammation.

Based on the results of this thesis, we conclude that the compounds formed during the 
MR have diverse effects on human GI health, from pro-inflammatory to anti-inflammatory and from anti-oxidative to pro-oxidative. Consequently, interference with anti-inflammatory drugs may occur. We propose that the ratio of different subsets that are formed through the MR might the in heated food products might determine the biological effects.

\section{Importance of starting product and thermal treatment}

The results of all our in vitro studies show that the cellular effects are largely dependent on both the starting product (specific carbohydrates and amino acids) and the thermal treatment of the product. The endogenous formation of AGEs in the GI tract in chapter 3 was dependent on the food product. In both the THP-1 and Caco-2 studies (chapter 2 and 4), the outcomes were dependent on thermal treatment: GC heated for 120 minutes significantly decreased cell viability of THP-1 and Caco-2 cells. In contrast, heating GC attenuated intracellular ROS production of unheated casein (UC) in Caco-2 cells. Evidence on the importance of starting product and thermal treatment on biological effects can be found in literature as well [7, 44]. Recently, this has been nicely demonstrated by some studies assessing the effect of processed food products on the human microbiota. Diet is known to have a large influence on the composition of the intestinal microbiota. One of the most well-known examples of the effect of diet on the microbiota is the difference in microbiome between breast-milk fed infants and infants fed with infant formula [45]. The microbiota of formula-fed infants has been shown to contain a decreased amount of bifidobacteria. Since bifidobacteria have been shown to have protective effects against pathogens and to fortify the intestinal immune system, a reduction in these bacteria may therefore compromise infant health $[45,46]$. Infant formula contains a substantial amount of dAGEs and since protein-bound dAGEs survive the GI tract, they could influence microbiota composition [47]. A recent review by Snelson et al. (2019) assessed the effects of dAGEs on gut microbiota regarding composition changes and SCFA production [48]. Concerning the microbiota composition, conflicting results between the in vitro and animal in vivo studies were found. Their conclusions however were that in animals, highdAGE diets were associated with a decrease in Bacteroidetes, bifidobacteria, lactobacilli and $\alpha$-diversity (the number of different species). It is generally considered that a decrease in all these three species and the $\alpha$ diversity leads to a less healthy GI tract. The effects of dAGEs on the microbiota seem to be dependent not only on the type of food or glycated material used, but also on biological differences between indivduals [31]. In general, human in vivo studies assessing the effect of dAGEs on microbiota are scarce. Most studies on the effect of dAGEs on the microbiota only used one type of food product or glycated material and one type of heating, which is not an optimal representation because treatment and starting product largely influence the outcome. This was additionally shown in a recent in vitro study by Pérez-Burillo et al. (2018), who analysed the effect of different foods products (chickpeas, bread, red pepper, banana, and chicken) on SCFA production [49]. All food products were prepared in different ways (i.e. roasted, fried, boiled, and grilled). The food products were digested with a static in vitro GI digestion model and consecutively fermented in vitro. After 
in vitro fermentation, SCFA production was analysed as well as the microbial community composition. Their main findings confirm that the effects on the microbiota both greatly depended on the type of food product and the cooking technique. It must be noted that the analyses of the food products focused on furosine (early glycation), and 5-(hydroxymethyl) furfural (HMF) (intermediate glycation), so the composition of specific dAGEs in the food products was not measured. It can be concluded that results of studies using only one type of starting product of thermal treatment should therefore be interpreted with caution and may explain the conflicting results found in literature concerning the effects of dAGEs.

\section{Exposure assessment}

Our results show that dAGEs formed during the MR have diverse effects on human GI health, depending on the way of food processing and the type of food product. The question that remains is to what extent humans are exposed to dAGEs. Multiple databases exist on the presence of different individual dAGEs in food products. Most of these databases measure only the presence of CML in food products. Several complications occur when assessing the intake of dAGEs using a database. Firstly, many food products have to be prepared, and the method of food preparation largely determines the levels of dAGEs. Some people eat their steaks 'raw' while others cook it for a longer time and enjoy theirs 'well done'. The effects of these preparation ways are however not included in the databases. Secondly, Niquet-Leridon et al. (2015) showed large differences in CML content between different brands of the same product. This might be due to use of different starting products, thermal treatment, and packaging [50]. Thirdly, as discussed before, many different dAGEs exist, and assessing the amount of one dAGE in a food product most likely does not give a proper representation of the total amount of dAGEs in the food product. Zhu et al. (2018) therefore proposed a panel of lysine-, arginine-, cysteine-, and nucleotide-derived dAGEs to estimate the amount of dAGEs in food products in a more accurate way [51]. Lastly, many of these databases make no distinction between protein-bound and free dAGEs, while our results show that this distinction is important to assess the possible effects. For future studies into the exposure of humans to dAGEs, it is therefore pivotal that a consensus should be made on the measuring techniques of $d A G E s$ and to acknowledge the problem that occurs with different food processing techniques.

Even though some concerns exist on the currently available dAGE databases, it is still interesting to have an indication on the contribution of specific food products to the daily dietary intake of dAGEs. Scheijen et al. (2016) analysed many commonly consumed Dutch food products on the presence of CML, CEL, and MG-H1 [52]. We calculated the intake of 3 dAGEs combined (CML, CEL, and MG-H1) per day per food item for the particular food item users from 19-30 years old in the Netherlands, based on the database published by Scheijen et al. (2016) and the Dutch National Food Consumption Survey [53]. Table 1 shows some examples of the food products within the top $15 \%$ range of the amount of dAGEs/day. The amounts of $\mathrm{CML}, \mathrm{CEL}$, and MG-H1 were summed up to give an overview on the total amount 
in the product.

Table 1. Average intake of $3 \mathrm{dAGEs}$ combined (CML, CEL, and MG-H1) per day per food item for the particular food item users from 19-30 years old in the Netherlands.

\begin{tabular}{llll}
\hline Food product & $\begin{array}{l}\text { Average intake } \\
\text { per day (g) [53] }\end{array}$ & $\begin{array}{l}\text { dAGEs content } \\
\text { (mg/100g) [52] }\end{array}$ & $\begin{array}{l}\text { Daily exposure to } \\
\text { dAGEs (mg/day) }\end{array}$ \\
\hline Blood sausages & 75.5 & 32 & 24.2 \\
\hline Beef steak (canned) & 18.7 & 78 & 14.6 \\
\hline Cereals (frosted flakes) & 27.1 & 39 & 10.5 \\
\hline Fried rice & 10.9 & 91 & 9.9 \\
\hline Peanut butter (Calve) & 51.5 & 18 & 9.3 \\
\hline Brown bread & 6.7 & 100 & 6.7 \\
\hline Peanuts & 31.7 & 21 & 6.7 \\
\hline Meat ball & 10.1 & 60 & 6.1 \\
\hline Chicken Wings & 4.6 & 127 & 5.9 \\
\hline Ginger biscuit & 32.8 & 12 & 3.9 \\
\hline
\end{tabular}

\section{Can this be clarified by the Maillard reaction?}

When looking at the top $15 \%$ of food products that contribute to the daily amount of ingested dAGEs in table 2, all products are subjected to thermal treatment during processing. Blood sausages, steak, and meat balls are all meat products containing proteins and sugars, as well as lipids that can contribute to the MR through the Namiki pathway. The cereals depicted are toasted corn topped with sugar syrup, leading to a faster reaction rate than normal cereals. Peanuts also contain 52\% fat, $26 \%$ protein, and $3.1 \%$ sugars and are roasted at high temperatures. Brown bread has a relatively low amount of dAGEs per $100 \mathrm{~g}$ compared to the other products, but the high consumption leads to a relatively high contribution to the total amount of dAGEs ingested per day. In general, the relatively high levels of dAGEs in these food products can therefore be clarified by the MR.

\section{Populations at risk}

Using the different dAGE databases and food consumption data from different population groups, it might be possible to distinguish groups of people that eat a lot of dAGEs and thus to define which people would be at higher risk for the intestinal effects of dAGEs. However, the effect of $d A G E s$ on the intestinal health of healthy people is currently unknown. Our results indicate that healthy people will most likely not experience adverse health effects by dAGEs, as no pro-inflammatory effect was seen in Caco-2 cells. In contrast, our results show that people who already have an inflamed GI tract, such as IBD patients, may experience negative effects from dAGEs. Although scientific evidence is scarce, diet is known to play a role in the symptoms of IBD. Commonly given diet-related advice for IBD patients includes avoidance of 
processed meat, fried foods, sugars and nuts [54,55]. Interestingly, exactly these products are also listed in our exposure assessment in table 1 and thus contain a lot of dAGEs. Based on the pro-inflammatory effect of dAGEs seen in this thesis, it seems logic that patients suffering from IBD will need to avoid these products as they may benefit from eating less dAGEs.

In addition to a possible correlation between the exposure to certain food groups and the presence of IBD symptoms, another important aspect in IBD is the composition and functionality of the gut microbiota. Studies into the effects of AGEs on the microbiota in relation to IBD are scarce. An in vitro study exposed faecal samples of healthy volunteers vs ulcerative colitis patients to native bovine serum albumin (BSA) and glycated-BSA (with glucose) [56]. When exposing the microbiota from healthy volunteers to glycated-BSA, the colonic microbiota started to reflect that of ulcerative colitis patients. Moreover, in both ulcerative colitis and non- ulcerative colitis microbiota, an increase in sulphate reducing bacteria was seen when exposed to glycated BSA versus native BSA. Within the ulcerative colitis samples, a significant increase was seen after exposure to glycated BSA compared to native BSA in the numbers of Clostridium perfringens/histolyticum group and Bacteroides spp. and a significant decrease in numbers of bifidobacteria and E. rectale group. These changes were not seen in the control model. No changes in the SCFA concentrations that could be attributed to any treatment were seen in the models [56]. These results indicate that dAGEs may influence the already impaired microbiota of IBD patents.

Irritable bowel syndrome (IBS) is a gastrointestinal disorder with frequent abdominal pain and changes in stool frequency and form, but no known underlying pathology or aetiology [57]. However, increased intestinal permeability and presence of activated immune cells in the GI tract have been found in IBS patients $[57,58]$. Similar to IBD, IBS patients often report the influence of different food products on their symptoms. Also here, these symptoms could be influenced by the extent to which patients are exposed to dAGEs. Based on our results, any person suffering from a disease or a drug side-effect that influences the GI epithelial layer or leads to macrophage presence in the GI tract, may suffer from consequences of a high-dAGE diet. In addition to IBD and IBS, these disorders include, but are not limited to: intestinal ischemia, chemotherapy-induced mucositis, NSAID-induced enteropathy, intestinal infections, celiac disease, food allergy, and heartburn [59]. As discussed before, low gastric $\mathrm{pH}$ is important to protein digestion and the dAGE-protein binding is important for the proinflammatory effects. Antacids neutralize the gastric acid to prevent heartburn, which might lead to even more protein-bound dAGEs in the GI tract [60].

Additionally, the use of specific food products as part of a specific diets may lead to a higher dAGE intake. For instance, lactose-free UHT milk may contain more dAGEs due to lactose being hydrolysed into glucose (reacts 10 times more rapidly than lactose) and galactose (reacts 20 times more rapidly than lactose) [61].

Finally, infants are a sensible group when it comes to GI health. During infancy, the GI tract and the immune system are still developing and have an important function in the development of allergies $[62,63]$. Infant formulas contain protein-bound dAGEs due to the high processing 
temperatures [64]. A study from 2008 showed 28 to 389 times more CML in infant formulas than breastmilk [47]. Hydrolysed infant formulas also contained significantly more CML than non-hydrolysed formula. Additionally, breast-fed infants showed approximately $46 \%$ lower plasma CML levels compared to the formula-fed infants, showing the uptake of CML into the systemic circulation in infants, although the majority of this CML was excreted via urine [47].

\section{Strategies to reduce dAGEs in food}

Due to the possible effects of dAGEs in certain populations, it is important to find strategies that might reduce dAGEs in food products or reduce their health effects. Lund et al. (2017) nicely reviewed dAGE reducing strategies [61]. We will therefore only give a small overview of the most promising strategies here. Some pharmaceutical strategies to influence the MR in both the human body and food have been investigated. Aminoguanidine has for instance been suggested as a promising pharmaceutical to trap $\alpha$-dicarbonyls in the early stages of the MR to prevent further formation of MRPs. However, clinical trials showed large side-effects and therefore this drug was abandoned. Next to pharmaceutical approaches, also foodbased strategies may be used to reduce dAGE production. In this regard, amines could be changed in food products by polyphenols to make them less reactive in the MR. Polyphenols, especially the oxidized quinones, can react with amines, thereby reducing dAGE formation [65]. However, this can influence protein functionality and may thereby reduce amino acid bioavailability. In addition to reducing dAGE formation, we have shown in chapter 5 that the polyphenol quercetin can also mitigate the negative effects of dAGEs endogenously. When it comes to trapping $\alpha$-dicarbonyls, both polyphenols like epicatechin and the B6 vitamer pyridoxamine have been shown to trap $\alpha$-dicarbonyls.

A different strategy currently approved in Canada is the use of hexose oxidases in UHT and sterilized milk. Hexose oxidases prevent the specific reducing sugars containing six carbon atoms from reacting in the MR. Since the MR can also be influenced by different conditions during thermal processing such as temperature, presence of diluents (e.g. water), and $\mathrm{H}+$ ions, this allows for many possibilities to mitigate dAGE formation during food processing [66]. 


\section{Conclusions, future perspectives, and implications}

In this thesis we investigated to what extent dAGEs may cause detrimental effects in the human GI tract. From the conducted studies, it can be concluded that dAGEs need to be protein-bound to exert a pro-inflammatory effect and that decreased digestibility of glycated proteins leads to the increased availability of protein-bound dAGEs throughout the complete GI tract. Moreover, besides the presence of dAGEs, also new endogenous AGEs are formed in the GI tract. Here in the GI tract, these AGEs are likely to exert pro-inflammatory effects, which may especially be detrimental in patients suffering from $\mathrm{Gl}$ diseases involving inflammation. However, more research is needed to elucidate the exact effects of dAGEs in the GI tract in humans. It would be important to assess a possible correlation between IBD and IBS symptoms and dAGE-intake. In case such a correlation is established, clinical studies could be set up in order to assess the influence of both high-dAGE and low-dAGE diets.

This thesis has additionally pointed out several pitfalls in existing dAGE research. Firstly, due to the formation of many different compounds during the MR, many effects seem food-type and thermal treatment dependent. This is an important aspect that is currently not taken into account by many dAGE-researchers and may explain, to at least some extent, the conflicting results of many in vitro and in vivo studies. It is therefore pivotal to characterise the dAGEs and food product-extracts used in studies. To go even further, food-based matrices may not be optimal means to study the effects of dAGEs as they are simplified food products and do not contain many components that in the actual food products may inhibit or stimulate the $M R$ as well as the biological effects. Although research of individual dAGEs is interesting from a mechanistic point of view, this cannot be used to draw conclusions on the overall effects of MRPs and food processing. Following up on this, we have shown that important differences are present between the inflammatory effects of protein-bound and free dAGEs, indicating that distinctions should be made between such dAGEs in assessing their biological effects. Finally, our studies into the effects of digestion on the pro-inflammatory effects and endogenous formation of dAGEs give an important implication for risk assessment of food products in general, namely that the effects of digestion on food components is very important for their toxicological effects. The endogenous formation of dAGEs during digestion implies that including digestion studies in food risk assessment is pivotal for correct exposure assessment and thus for the correct assessment of potential risks. 


\section{References}

1. Kislinger, T., et al., N(epsilon)-(carboxymethyl)lysine adducts of proteins are ligands for receptor for advanced glycation end products that activate cell signaling pathways and modulate gene expression. J Biol Chem, 1999. 274(44): p. 31740-9.

2. Buetler, T.M., et al., N-epsilon-carboxymethyllysine-modified proteins are unable to bind to RAGE and activate an inflammatory response. Molecular Nutrition \& Food Research, 2008. 52(3): p. 370-378.

3. Xie, J., et al., Structural basis for pattern recognition by the receptor for advanced glycation end products (RAGE). J Biol Chem, 2008. 283(40): p. 27255-69.

4. Xue, J., et al., Advanced glycation end product recognition by the receptor for AGEs. Structure, 2011. 19(5): p. 722-32.

5. Xue, J., et al., The receptor for advanced glycation end products (RAGE) specifically recognizes methylglyoxal-derived AGEs. Biochemistry, 2014. 53(20): p. 3327-35.

6. Chen, Y. and T.L. Guo, Dietary Early Glycation Products Promote the Growth of Prostate Tumors More than Advanced Glycation End-Products through Modulation of Macrophage Polarization. Mol Nutr Food Res, 2019. 63(4): p. e1800885.

7. Oh, J.G., et al., Anti-inflammatory effect of sugar-amino acid Maillard reaction products on intestinal inflammation model in vitro and in vivo. Carbohydr Res, 2017. 449: p. 47-58.

8. Chen, X.M. and D.D. Kitts, Antioxidant and anti-inflammatory activities of Maillard reaction products isolated from sugar-amino acid model systems. J Agric Food Chem, 2011. 59(20): p. 11294-303.

9. Guerra, A., et al., Relevance and challenges in modeling human gastric and small intestinal digestion. Trends in Biotechnology, 2012. 30(11): p. 591-600.

10. Sams, L., et al., Relevant $\mathrm{pH}$ and lipase for in vitro models of gastric digestion. Food Funct, 2016. 7(1): p. 30-45.

11. Hellwig, M., et al., N-epsilon-fructosyllysine and N-epsilon-carboxymethyllysine, but not Iysinoalanine, are available for absorption after simulated gastrointestinal digestion. Amino Acids, 2014. 46(2): p. 289-99.

12. Stanstrup, J., et al., Whey protein delays gastric emptying and suppresses plasma fatty acids and their metabolites compared to casein, gluten, and fish protein. J Proteome Res, 2014. 13(5): p. 2396-408.

13. Read, N.W., et al., Transit of a meal through the stomach, small intestine, and colon in normal subjects and its role in the pathogenesis of diarrhea. Gastroenterology, 1980. 79(6): p. $1276-82$.

14. Joubran, Y., A. Moscovici, and U. Lesmes, Antioxidant activity of bovine alpha lactalbumin Maillard products and evaluation of their in vitro gastro-duodenal digestive proteolysis. Food Funct, 2015. 6(4): p. 1229-40.

15. Zhao, D., et al., Digestibility of Glyoxal-Glycated beta-Casein and beta-Lactoglobulin and Distribution of Peptide-Bound Advanced Glycation End Products in Gastrointestinal Digests. J Agric Food Chem, 2017. 65(28): p. 5778-5788.

16. Pinto, M.S., et al., Heating and glycation of beta-lactoglobulin and beta-casein: Aggregation and in vitro digestion. Food Research International, 2014. 55: p. 70-76.

17. Moscovici, A.M., et al., The impact of the Maillard reaction on the in vitro proteolytic breakdown of bovine lactoferrin in adults and infants. Food Funct, 2014. 5(8): p. 1898-908.

18. van Lieshout, G.A.A., et al., How processing may affect milk protein digestion and overall physiological outcomes: A systematic review. Crit Rev Food Sci Nutr, 2019: p. 1-24.

19. Oliver, C.M., L.D. Melton, and R.A. Stanley, Creating proteins with novel functionality via the Maillard reaction: a review. Crit Rev Food Sci Nutr, 2006. 46(4): p. 337-50.

20. Wada, Y. and B. Lonnerdal, Effects of Different Industrial Heating Processes of Milk on SiteSpecific Protein Modifications and Their Relationship to in Vitro and in Vivo Digestibility. Journal of Agricultural and Food Chemistry, 2014. 62(18): p. 4175-4185.

21. Joubran, Y., et al., Implications of the Maillard reaction on bovine alpha-lactalbumin and its proteolysis during in vitro infant digestion. Food Funct, 2017. 8(6): p. 2295-2308.

22. Nyakayiru, J., et al., The glycation level of milk protein strongly modulates post-prandial lysine availability in humans. Br J Nutr, 2019: p. 1-22. 
23. Bains, Y., A. Gugliucci, and R. Caccavello, Advanced glycation endproducts form during ovalbumin digestion in the presence of fructose: Inhibition by chlorogenic acid. Fitoterapia, 2017. 120: p. 1-5.

24. DeChristopher, L.R., J. Uribarri, and K.L. Tucker, The link between soda intake and asthma: science points to the high-fructose corn syrup, not the preservatives: a commentary. Nutr Diabetes, 2016. 6(11): p. e234.

25. DeChristopher, L.R., Perspective: The Paradox in Dietary Advanced Glycation End Products Research-The Source of the Serum and Urinary Advanced Glycation End Products Is the Intestines, Not the Food. Advances in Nutrition, 2017. 8(5): p. 679-683.

26. Ferraris, R.P., J.Y. Choe, and C.R. Patel, Intestinal Absorption of Fructose. Annu Rev Nutr, 2018. 38: p. 41-67.

27. Jones, H.F., R.N. Butler, and D.A. Brooks, Intestinal fructose transport and malabsorption in humans. Am J Physiol Gastrointest Liver Physiol, 2011. 300(2): p. G202-6.

28. Gouyon, F., et al., Simple-sugar meals target GLUT2 at enterocyte apical membranes to improve sugar absorption: a study in GLUT2-null mice. J Physiol, 2003. 552(Pt 3): p. 823-32.

29. Oimomi, M., et al., Fructose-related glycation. Diabetes Res Clin Pract, 1989. 7(2): p. 137-9.

30. Martinez-Saez, N., et al., In vitro formation of Maillard reaction products during simulated digestion of meal-resembling systems. Food Res Int, 2019. 118: p. 72-80.

31. Helou, C., et al., Insights into bread melanoidins: fate in the upper digestive tract and impact on the gut microbiota using in vitro systems. Food \& Function, 2015. 6(12): p. 3737-3745.

32. Delgado-Andrade, C. and V. Fogliano, Dietary Advanced Glycosylation End-Products (dAGEs) and Melanoidins Formed through the Maillard Reaction: Physiological Consequences of their Intake. Annu Rev Food Sci Technol, 2018. 9: p. 271-291.

33. Yáñez, D.A.C., et al., Antioxidant activity developed at the different stages of Maillard reaction with milk proteins. LWT- Food Science and Technology, 2018(89): p. 344-349.

34. Patrignani, M., et al., Antioxidant capacity of Maillard reaction products in the digestive tract: An in vitro and in vivo study. Food Chemistry, 2019. 276: p. 443-450.

35. Chuyen, N.V., et al., Antioxidative properties of products from amino acids or peptides in the reaction with glucose. Adv Exp Med Biol, 1998. 434: p. 201-12.

36. Borrelli, R.C. and V. Fogliano, Bread crust melanoldins as potential prebiotic ingredients. Molecular Nutrition \& Food Research, 2005. 49(7): p. 673-678.

37. Gilbert, M.S., et al., Protein fermentation in the gut; implications for intestinal dysfunction in humans, pigs, and poultry. Am J Physiol Gastrointest Liver Physiol, 2018. 315(2): p. G159-G170.

38. Tuohy, K.M., et al., Metabolism of Maillard reaction products by the human gut microbiota-implications for health. Mol Nutr Food Res, 2006. 50(9): p. 847-57.

39. Vinolo, M.A., et al., Regulation of inflammation by short chain fatty acids. Nutrients, 2011. 3(10): p. 858-76.

40. Li, M., et al., The Anti-inflammatory Effects of Short Chain Fatty Acids on Lipopolysaccharideor Tumor Necrosis Factor alpha-Stimulated Endothelial Cells via Activation of GPR41/43 and Inhibition of HDACs. Front Pharmacol, 2018. 9: p. 533.

41. Barnes, P.J., Mechanisms and resistance in glucocorticoid control of inflammation. J Steroid Biochem Mol Biol, 2010. 120(2-3): p. 76-85.

42. Tung, J., et al., A population-based study of the frequency of corticosteroid resistance and dependence in pediatric patients with Crohn's disease and ulcerative colitis. Inflamm Bowel Dis, 2006. 12(12): p. 1093-100.

43. Munkholm, P., et al., Frequency of glucocorticoid resistance and dependency in Crohn's disease. Gut, 1994. 35(3): p. 360-2.

44. Wijewickreme, A.N. and D.D. Kitts, Influence of reaction conditions on the oxidative behavior of model Maillard-reaction products. Journal of Agricultural and Food Chemistry, 1997. 45(12): p. 4571-4576.

45. Albenberg, L.G. and G.D. Wu, Diet and the intestinal microbiome: associations, functions, and implications for health and disease. Gastroenterology, 2014. 146(6): p. 1564-72.

46. Fukuda, S., et al., Bifidobacteria can protect from enteropathogenic infection through production of acetate. Nature, 2011. 469(7331): p. 543-7.

47. Sebekova, K., et al., Plasma concentration and urinary excretion of N-epsilon(carboxymethyl)lysine in breast milk- and formula-fed infants. Maillard Reaction: 
Recent Advances in Food and Biomedical Sciences, 2008. 1126: p. 177-180.

48. Snelson, M. and M.T. Coughlan, Dietary Advanced Glycation End Products: Digestion, Metabolism and Modulation of Gut Microbial Ecology. Nutrients, 2019. 11(2).

49. Perez-Burillo, S., et al., Effect of Food Thermal Processing on the Composition of the Gut Microbiota. J Agric Food Chem, 2018. 66(43): p. 11500-11509.

50. Niquet-Leridon, C., et al., The rehabilitation of raw and brown butters by the measurement of two of the major Maillard products, N(epsilon)-carboxymethyl-lysine and

5-hydroxymethylfurfural, with validated chromatographic methods. Food Chem, 2015. 177: p. 361-8.

51. Zhu, Y., H. Snooks, and S. Sang, Complexity of Advanced Glycation End Products in Foods: Where Are We Now? J Agric Food Chem, 2018. 66(6): p. 1325-1329.

52. Scheijen, J., et al., Analysis of advanced glycation endproducts in selected food items by ultra-performance liquid chromatography tandem mass spectrometry: Presentation of a dietary AGE database. Food Chem, 2016. 190: p. 1145-1150.

53. (RIVM), N.I.f.P.H.a.t.E., Dutch National Food Consumption Survey 2009, www. voedselconsumptiepeiling.nl (version 11-2009): The Hague, The Netherlands.

54. Hou, J.K., D. Lee, and J. Lewis, Diet and inflammatory bowel disease: review of patienttargeted recommendations. Clin Gastroenterol Hepatol, 2014. 12(10): p. 1592-600.

55. Rizzello, F., et al., Implications of the Westernized Diet in the Onset and Progression of IBD. Nutrients, 2019. 11(5).

56. Mills, D.J.S., et al., Dietary glycated protein modulates the colonic microbiota towards a more detrimental composition in ulcerative colitis patients and non-ulcerative colitis subjects. Journal of Applied Microbiology, 2008. 105(3): p. 706-714.

57. Camilleri, M., K. Lasch, and W. Zhou, Irritable bowel syndrome: methods, mechanisms, and pathophysiology. The confluence of increased permeability, inflammation, and pain in irritable bowel syndrome. Am J Physiol Gastrointest Liver Physiol, 2012. 303(7): p. G775-85.

58. Barbara, G., et al., A role for inflammation in irritable bowel syndrome? Gut, 2002. 51 Suppl 1: p. i41-4.

59. Catalioto, R.M., C.A. Maggi, and S. Giuliani, Intestinal epithelial barrier dysfunction in disease and possible therapeutical interventions. Curr Med Chem, 2011. 18(3): p. 398-426.

60. Nederland, Z. Farmacotherapeutisch Kompas. 2020 [cited 20201 March]; Available from: https://www.farmacotherapeutischkompas.nl/bladeren/indicatieteksten/functionele _ maagklachten.

61. Lund, M.N. and C.A. Ray, Control of Maillard Reactions in Foods: Strategies and Chemical Mechanisms. J Agric Food Chem, 2017. 65(23): p. 4537-4552.

62. Smith, P.K., et al., The false alarm hypothesis: Food allergy is associated with high dietary advanced glycation end-products and proglycating dietary sugars that mimic alarmins. J Allergy Clin Immunol, 2017. 139(2): p. 429-437.

63. Teodorowicz, M., J. van Neerven, and H. Savelkoul, Food Processing: The Influence of the Maillard Reaction on Immunogenicity and Allergenicity of Food Proteins. Nutrients, 2017. 9(8).

64. Penndorf, I., et al., Studies on N-terminal glycation of peptides in hypoallergenic infant formulas: quantification of alpha-N-(2-furoylmethyl) amino acids. J Agric Food Chem, 2007. 55(3): p. 723-7.

65. Yin, J., et al., Epicatechin and epigallocatechin gallate inhibit formation of intermediary radicals during heating of lysine and glucose. Food Chem, 2014. 146: p. 48-55.

66. Helou, C., et al., The impact of raw materials and baking conditions on Maillard reaction products, thiamine, folate, phytic acid and minerals in white bread. Food Funct, 2016. 7(6): p. 2498-507. 

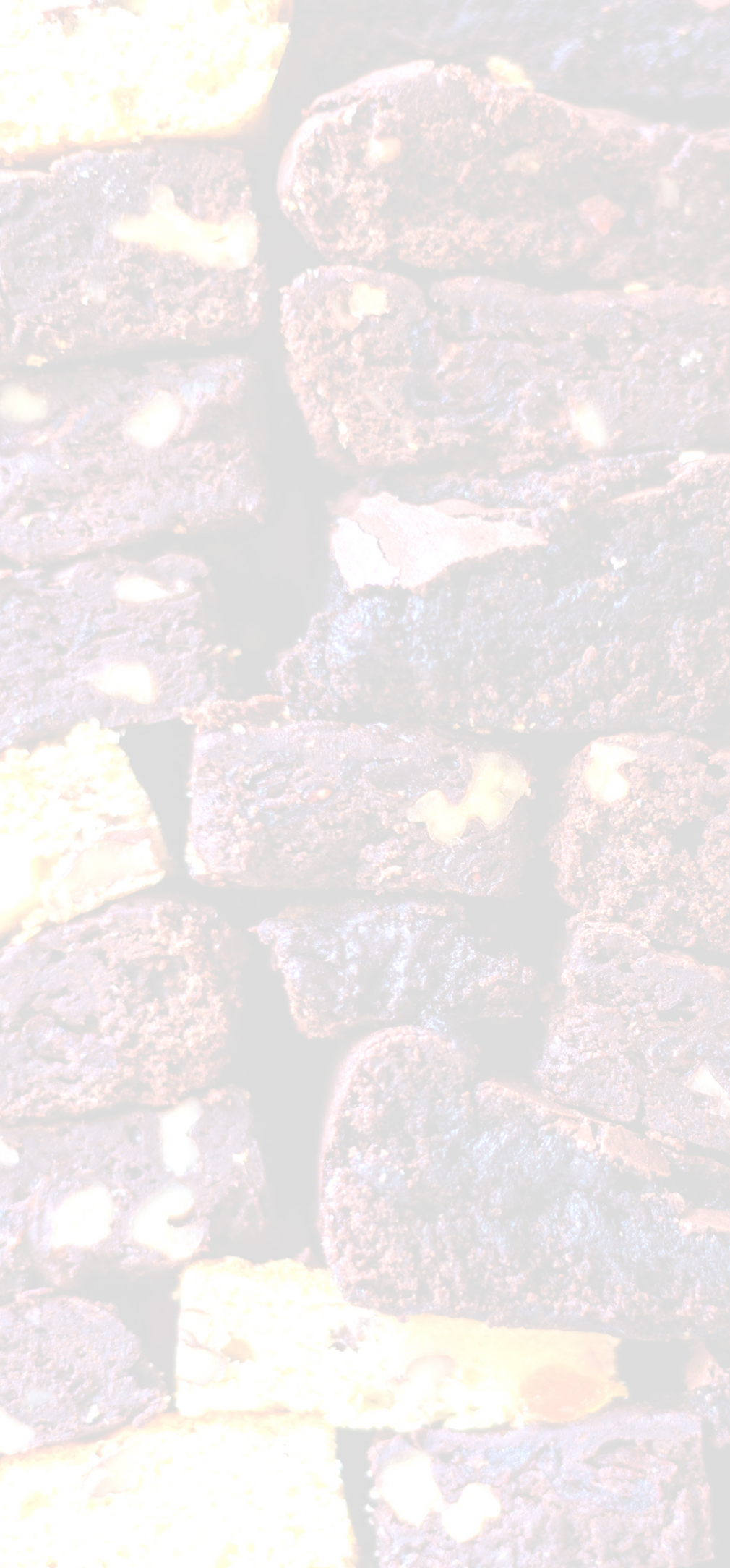


\section{Addendum}

Samenvatting

Valorisatie

Dankwoord

Curriculum Vitae

List of publications 


\section{Samenvatting}

Tijdens de bereiding van voedsel, zowel in de fabriek als in de keuken, wordt voedsel blootgesteld aan hoge temperaturen. Deze hoge temperaturen zorgen voor het verloop van een chemische reactie, genaamd de Maillard reactie. De Maillard reactie begint met de reactie tussen suikers en eiwitten, welke Schiff-basen vormt. Deze Schiff-basen reageren verder via meerdere opeenvolgende reacties tot advanced glycation endproducts (AGEs), een diverse groep moleculen die grotendeels bruin van kleur zijn. AGEs zijn volop aanwezig in bereid voedsel, te zien aan de bruinkleuring. De Maillard reactie verloopt ook in het lichaam, bij $37^{\circ} \mathrm{C}$, met endogene suikers en eiwitten. In het lichaam zorgen deze endogene AGEs voor verschillende effecten, waaronder het veroorzaken en verergeren van ontstekingen. Ondanks deze kennis is het tot op heden niet bekend in hoeverre voedings-AGEs effect hebben in het menselijk lichaam. Via ons voedsel komen er veel voedings-AGEs terecht in het lichaam en voornamelijk in het maag-darmkanaal. De effecten van deze voedings-AGEs in de darmen zijn nog nauwelijks onderzocht. Het doel van dit proefschrift is daarom om de mogelijke proinflammatoire (ontstekingsbevorderende) effecten van voedings-AGEs in het darmstelsel te onderzoeken en te inventariseren welke verschillende Nederlandse populatiegroepen vooral last kunnen hebben van deze effecten van voedings-AGEs.

\section{Voedings-AGEs en ontstekingsreacties}

Endogene AGEs veroorzaken ontstekingsreacties door het activeren van de receptor voor advanced glycation endproducts (RAGE). Activatie van RAGE zorgt voor de secretie van proinflammatoire cytokines, zoals tumornecrosefactor- $\alpha$ (TNF- $\alpha$ ) en interleukine-8 (IL-8). In hoofdstuk 2 hebben we humane macrofagen blootgesteld aan verschillende voedings-AGEs, waarna we de secretie van TNF- $\alpha$ en celdood gemeten hebben. De verschillende voedingsAGEs bestonden uit eiwitgebonden AGEs en vrije AGEs. De eiwitgebonden AGEs werden in de vorm van geglyceerd caseïne (GC) geproduceerd door het verhitten van caseïne, lactose, en glucose in een fosfaatbuffer op $100^{\circ} \mathrm{C}$ gedurende 15 tot 120 minuten. De verschillende vrije AGEs waren: $\mathrm{N} \varepsilon$-carboxymethyllysine (CML), NE-carboxyethyllysine (CEL), methylglyoxalderived hydroimidazolone 1 (MG-H1), en acrylamide. De humane macrofagen produceerden TNF- $\alpha$ na blootstelling aan GC, de eiwitgebonden AGEs, op een dosis-afhankelijke wijze. De verschillende vrije AGEs veroorzaakten geen TNF- $\alpha$ secretie. Dit duidt op een belangrijke rol voor de eiwitbinding van AGEs als het gaat om de pro-inflammatoire effecten. Naast het proinflammatoire effect zorgde een langere verhittingstijd van GC tot een verhoogde celdood van de macrofagen. Het blokkeren van RAGE met een antagonist leidde tot een significante reductie van TNF- $\alpha$ secretie, wat duidt op een RAGE-afhankelijk effect van eiwitgebonden voedings-AGEs.

\section{Vertering van AGEs}

Voordat voedings-AGEs in het lichaam terechtkomen, worden ze eerst verteerd in het maag-darmkanaal. In hoofdstuk 2 hebben we laten zien dat het pro-inflammatoire effect 
van voedings-AGEs afhankelijk is van de AGE-eiwitbinding. Tijdens de maag-darmpassage van voedingsmiddelen worden eiwitten afgebroken tot aminozuren, dit zou kunnen leiden tot de afbraak van de AGE-eiwitbinding, waardoor de voedings-AGEs een verminderd pro-inflammatoir effect zouden kunnen hebben. In hoofdstuk 3 hebben we verschillende voedingsproducten die rijk zijn aan voedings-AGEs (GC, speculaas koekjes en appelsap) blootgesteld aan een geavanceerd in vitro maag- en dunne darm model: het TNO gastrolntestinal Model-1 (TIM-1). Dit model bootst het maagdarmkanaal na en houdt rekening met meerdere parameters, zoals: dynamische pH-curven, peristaltiek, toevoeging van galen pancreasenzymen, en passieve absorptie. Tijdens de in vitro vertering van GC, speculaas koekjes, en appelsap bleef de AGE-eiwitbinding intact. Dit betekent dat voedings-AGEs hun pro-inflammatoire eigenschappen behouden in de dunne darm. Als we de vertering van GC vergelijken met de vertering van ongeglyceerde eiwitten (UC), blijkt dat de aanwezigheid van eiwitgebonden AGEs de eiwit vertering vertraagt. Dit kan de biologische beschikbaarheid van essentiële en non-essentiële aminozuren verlagen en daarmee consequenties hebben voor de menselijke gezondheid. Daarnaast laten we zien dat tijdens de vertering van speculaas koekjes en appelsap nieuwe AGEs worden gevormd in de dunne darm. Deze kunnen bijdragen aan de pro-inflammatoire effecten. Dit effect lijkt ook product-afhankelijk te zijn, de toenames waren verschillend tussen de verschillende voedingsproducten.

\section{Effecten van voedings-AGEs in het maag-darmkanaal}

Om het effect van vertering op het pro-inflammatoire potentieel van voedings-AGEs te onderzoeken, hebben we in hoofdstuk 4 humane macrofagen blootgesteld aan de door TIM1 verteerde GC. Hieruit bleek dat de verteerde GC voor meer TNF- $\alpha$ secretie zorgt dan de onverteerde GC. Om te onderzoeken in hoeverre de grootte van de moleculen effect heeft op het pro-inflammatoir potentieel van voedings-AGEs zijn de onverteerde GC-samples gefractioneerd op grootte en zijn de humane macrofagen blootgesteld aan deze individuele fracties. Hier zien we dat de grootste moleculen in GC voor de meeste TNF- $\alpha$ secretie veroorzaken, terwijl de kleinste moleculen geen TNF- $\alpha$ secretie veroorzaken. Om het effect van voedings-AGEs in het maag-darmkanaal verder te onderzoeken hebben we darmepitheel cellen blootgesteld aan GC. Hoe langer de verhittingstijd van GC, hoe meer darmepitheel cellen doodgingen. In tegenstelling tot deze bevinding, zagen we ook dat hoe langer GC verhit is, hoe lager de productie van intracellulaire reactieve zuurstofcomponenten (iROS) door darmepitheel cellen was in vergelijking tot onverhit GC. Met deze resultaten laten we zien dat de specifieke thermische behandeling van voedingsproducten een belangrijke rol speelt in de uiteindelijke waargenomen biologische effecten van de voedings-AGEs.

\section{AGEs en corticosteroïdengevoeligheid}

Patiënten met inflammatoire darmziekten (IBD) zoals de ziekte van Crohn en Colitis Ulcerosa lijden aan chronische ontstekingsreacties in hun maag-darmkanaal. Om deze ontstekingen te behandelen krijgen deze patiënten corticosteroïden toegediend. Corticosterö̈den zijn een van 
de meest gebruikte anti-inflammatoire medicijnen, maar een groot deel van IBD-patiënten is (deels) resistent tegen deze medicijnen. In hoofdstuk $\mathbf{5}$ hebben we onderzocht in hoeverre voedings-AGEs de corticosteroïdengevoeligheid kan beïnvloeden in humane macrofagen. In dit hoofdstuk laten we zien dat GC de corticosteroïden-gevoeligheid van macrofagen verlaagt wat zou kunnen leiden tot corticosteroïden-resistentie. Deze ongevoeligheid kan worden gereduceerd door de suppletie met het antioxidant quercetine. Deze verhoging van corticosteroïde-gevoeligheid door quercetine komt waarschijnlijk door het wegvangen van iROS door dit antioxidant. Hiermee laten we zien dat voedingsproducten met een hoog pro-inflammatoir potentieel corticosteroïden-resistentie kan veroorzaken. Deze resultaten kunnen van groot belang zijn voor IBD-patiënten die een verlaagde corticosteroïdengevoeligheid hebben.

\section{Algemene conclusie}

Het huidige Westerse dieet zorgt ervoor dat mensen veelal worden blootgesteld aan voedings-AGEs. Deze voedings-AGEs worden voornamelijk gevormd tijdens de bereiding van het voedsel, waarbij temperatuur een belangrijke rol speelt. Voedings-AGEs veroorzaken een ontstekingsreactie in humane macrofagen. Voedings-AGEs zijn daarom een potentieel risico voor patiënten met inflammatoire darmaandoeningen. Om een pro-inflammatoir effect te kunnen uitoefenen moeten voedings-AGEs eiwitgebonden zijn, het verteringsproces van het maag-darmkanaal verbreekt deze AGE-eiwitbinding niet en er worden zelfs meer eiwitgebonden AGEs gevormd in het darmkanaal tijdens de vertering van voedingsproducten met hoge concentraties voedings-AGEs. Hierdoor blijven voedings-AGEs pro-inflammatoir tijdens en na de vertering. De duur van het verhittingsproces en de type en hoeveelheid suiker en eiwitten in de voeding zijn erg belangrijk voor de uiteindelijke biologische effecten van voedings-AGEs. Uit ons onderzoek komt naar voren dat voornamelijk mensen met inflammatoire darmziektes baat zouden kunnen hebben bij het verminderen van hun voedings-AGE-inname. Dit met als doel om hun symptomen te verminderen. 

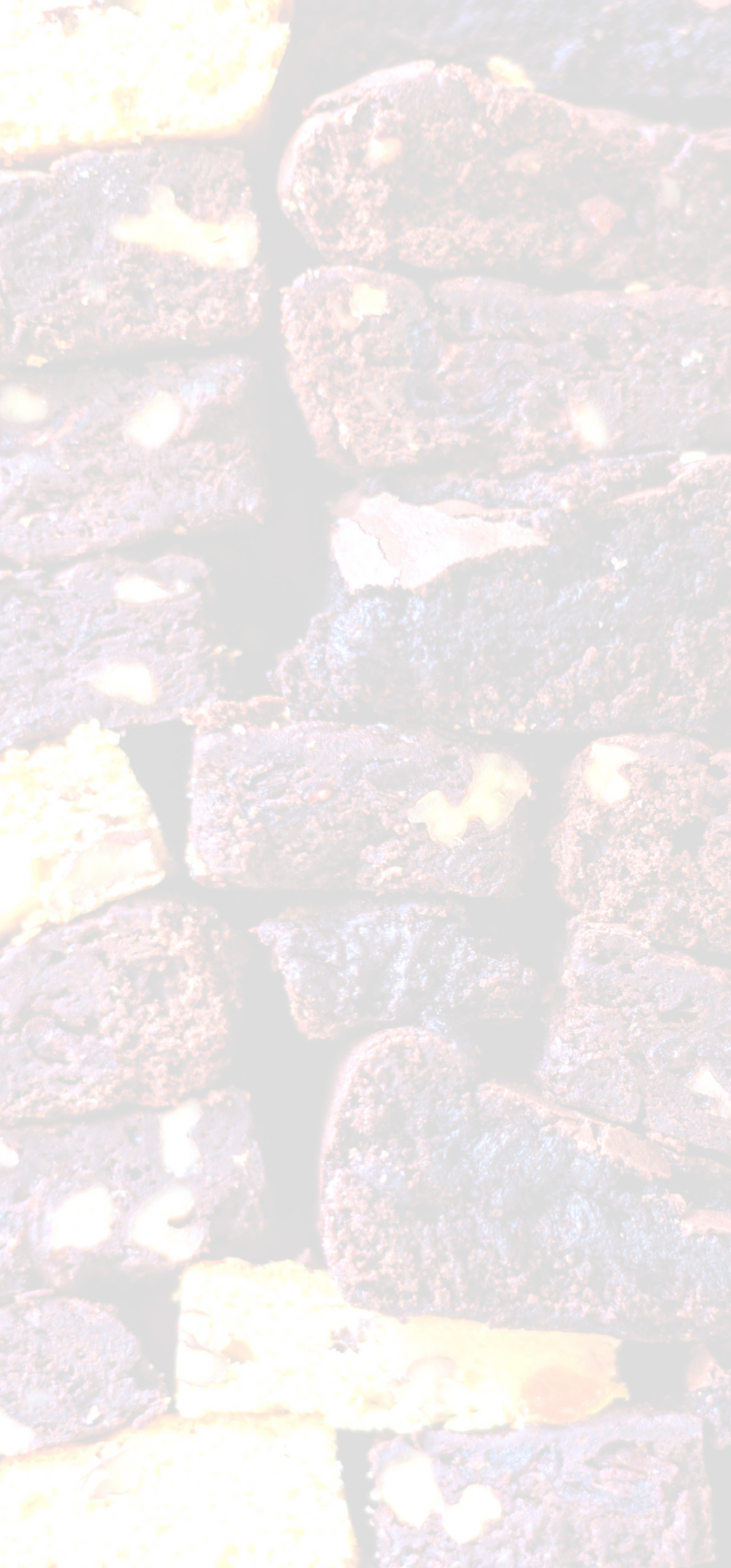


\title{
Addendum
}

\author{
Samenvatting \\ Valorisatie \\ Dankwoord \\ Curriculum Vitae \\ List of publications
}




\section{Valorisatie}

Het verhitten van voedsel is erg belangrijk voor de mensheid. Het zorgt voor het doden van pathogene organismen waarmee de voedselveiligheid verbeterd en stoffen kunnen worden vrijgemaakt of gevormd zoals bijvoorbeeld antioxidanten [1].

Het verhitten van voedsel kan ook voor ongezonde effecten zorgen. Een bekend voorbeeld is het verbranden van voedsel, hierdoor worden kankerverwekkende stoffen gevormd. Maar niet alleen het zwarte, verbrande deel van voedsel kan een negatief effect hebben. Het verhitten van voedsel leidt tot het verlopen van de Maillard reactie, een chemische reactie tussen suikers en eiwitten die versneld wordt door hoge temperaturen. Tijdens deze Maillard reactie worden bruinkleurige moleculen genaamd advanced glycation endproducts (AGEs) gevormd. In dit proefschrift hebben we laten zien dat deze voedings-AGEs verschillende biologische effecten kunnen hebben.

Het is tot op heden onbekend in hoeverre voedings-AGEs worden opgenomen in het lichaam. In dit proefschrift hebben we laten zien dat voedings-AGEs in de dunne en dikke darm terechtkomen in een pro-inflammatoire vorm. Deze informatie kan van belang zijn voor verschillende populatiegroepen. Ten eerste patiënten die lijden aan inflammatoire darmziektes zoals de ziekte van Crohn (CD) en colitis ulcerosa (UC). Deze ziektebeelden vallen onder de paraplu 'inflammatory bowel disease (IBD)'. IBD lijdt tot ontstoken secties van het maagdarmstelsel door een onbekende oorzaak. De ziekte verlaagt de kwaliteit van leven van patiënten en gaat gepaard met veel pijn en verteringsproblemen [2]. De afgelopen decennia is de incidentie van IBD in Zuid-Limburg erg gestegen, van 17.90 per 100.000 inwoners in 1991 naar 40.36 per 100.000 inwoners in 2010 [2]. In 2010 was de geschatte prevalentie van IBD in Nederland 613 per 100.000 inwoners [2]. De incidentie en prevalentie blijft momenteel stijgen in heel Europa [3]. Deze stijgingen kunnen verklaard worden door verbeterde diagnostische technieken, maar ook door omgevingsfactoren zoals voeding. IBD heeft een grote impact op de levenskwaliteit van de patiënt, maar heeft ook socio-economische consequenties. De ziekte wordt behandeld door middel van anti-inflammatoire medicijnen, en in het uiterste geval wordt een deel van de darm chirurgisch verwijderd. Binnen Europa heeft uiteindelijk 30-50\% van de IBD patiënten een chirurgische ingreep nodig [3]. Burisch et al. (2013) schatte de totale, directe, ziektekosten in Europa op 4.6 - 5.6 miljoen Euro. Naast deze ziektekosten is er ook relatief veel ziekteverzuim door IBD-patiënten en arbeidsongeschiktheid. IBDpatiënten ervaren verschillende periodes van remissies en oplaaiingen van symptomen. Vooral bij deze symptomen zou het verminderen van voedings-AGEs kunnen helpen. Een groep anti-inflammatoire medicijnen die veel aan IBD-patiënten wordt voorgeschreven zijn corticosteroïden. Opmerkelijk genoeg is een relatief groot percentage, ongeveer $20 \%$, van IBD-patiënten in zekere mate ongevoelig voor corticosteroïden [4, 5]. En de onderliggende oorzaak hiervan is tot op heden nog niet gevonden. Dit proefschrift laat zien dat voedingsAGEs de potentie hebben om deze ongevoeligheid te veroorzaken of in ieder geval te vergroten. Een dieet laag in voedings-AGEs en hoog in polyfenolen zou de corticosteroïde- 
gevoeligheid kunnen verhogen in deze patiënten.

Naast het potentiele effect van voedings-AGEs op de symptomen van IBD-patiënten kunnen voedings-AGEs ook de vertering van eiwitten beïnvloeden en daarmee de biologische beschikbaarheid van aminozuren beïnvloeden. AGEs worden ook gevormd in het darmstelsel. Deze resultaten zijn belangrijk voor de ontwikkeling van gezondere voedingsproducten door de levensmiddelenindustrie. Die hun bereidingsmethodes en grondstoffen kunnen aanpassen.

Dit proefschrift laat zien dat er een aantal factoren zijn die belangrijk zijn voor de vorming van voedings-AGEs in voeding bij het maken en bewerken van deze voedingsmiddelen in de fabriek. Uiteraard ten eerste de temperatuur waarop voedingsmiddelen worden bereid, hoe lager de temperatuur, hoe minder voedings-AGEs er gevormd worden. Ook hebben we laten zien dat de biologische effecten en vorming van voedings-AGEs afhankelijk kunnen zijn van de voedings-matrix, hoogstwaarschijnlijk de verschillen in suikers en eiwitten. Fructose lijkt vele malen reactiever in de Maillard reactie dan bijvoorbeeld glucose. Glucosefructosestroop is een veel gebruikt zoetmiddel in de voedingsmiddelenindustrie, dit gebruik inperken zou eventueel voor minder inname van voedings-AGEs door consumenten kunnen zorgen. Ook kunnen eventueel de precursors van AGEs zoals glyoxal en methylglyoxal in eten verminderd kunnen worden door het aanpassen van de industriële bereidingsmethodes. Deze aanknopingspunten kunnen bijvoorbeeld gebruikt worden door de Nederlandse Voedsel- en Warenautoriteit (NVWA) voor verder verdiepend onderzoek. De NVWA kan dan deze informatie gebruiken om indien nodig een advies te schrijven voor de overheid.

Naast de rol van levensmiddelenproducenten in het verminderen van de voedings-AGEs inname kunnen consumenten ook gezondere bereidingsmethodes in de keuken toepassen. Naast het aanpassen van bereidingsmethodes kunnen verschillende stoffen aan het eten toegevoegd worden om de voedings-AGE-vorming en biologische effecten te verminderen. Polyfenolen zoals quercetine en epicatechine kunnen hierin van pas komen.

\section{Referenties}

1. Miglio, C., et al., Effects of different cooking methods on nutritional and physicochemical characteristics of selected vegetables. J Agric Food Chem, 2008. 56(1): p. 139-47.

2. van den Heuvel, T.R.A., et al., A 20-Year Temporal Change Analysis in Incidence, Presenting Phenotype and Mortality, in the Dutch IBDSL Cohort-Can Diagnostic Factors Explain the Increase in IBD Incidence? J Crohns Colitis, 2017. 11(10): p. 1169-1179.

3. Burisch, J., et al., The burden of inflammatory bowel disease in Europe. J Crohns Colitis, 2013. 7(4): p. 322-37.

4. Tung, J., et al., A population-based study of the frequency of corticosteroid resistance and dependence in pediatric patients with Crohn's disease and ulcerative colitis. Inflamm Bowel Dis, 2006. 12(12): p. 1093-100.

5. Munkholm, P., et al., Frequency of glucocorticoid resistance and dependency in Crohn's disease. Gut, 1994. 35(3): p. 360-2. 

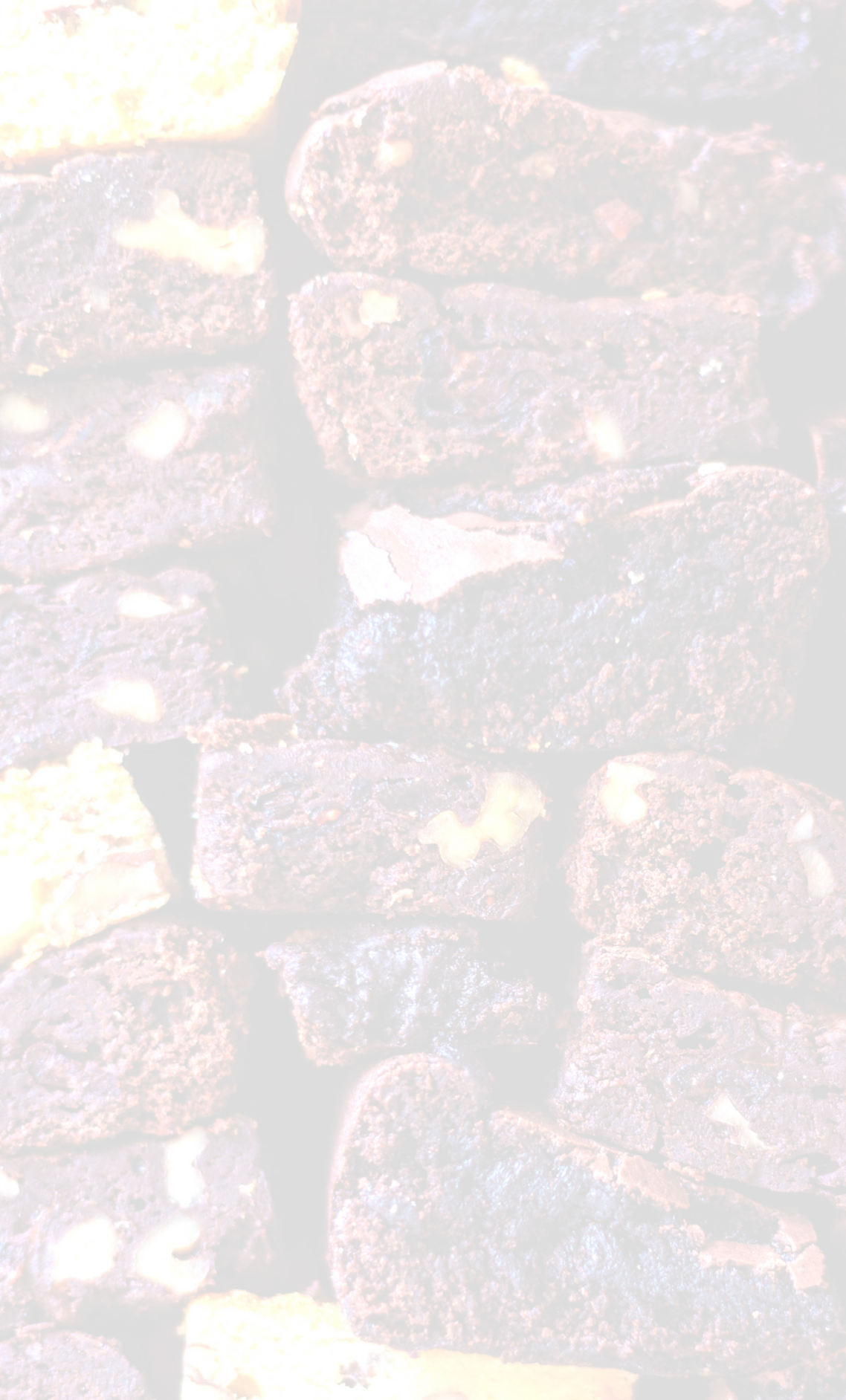


\title{
Addendum
}

\author{
Samenvatting \\ Valorisatie \\ Dankwoord \\ Curriculum Vitae \\ List of publications
}




\section{Dankwoord}

Lieve papa,

Eigenlijk begon dit dankwoord heel anders, over hoe ik 5 jaar geleden begon aan een groot avontuur en dat ik graag iedereen wilde bedanken die daar bij betrokken is geweest.

24 juni 2020, een week voordat ik dit boekje naar de drukker wilde sturen, werd onze hele wereld op z'n kop gegooid. Het doet me verschrikkelijk veel pijn en verdriet te bedenken dat jij er niet zal zijn bij mijn promotie, om jouw trotse hoofd daar niet te zien zitten op de eerste rij.

Zonder de kwaliteiten die je mij hebt doorgegeven had ik dit traject niet volbracht en was ik waarschijnlijk niet eens op het idee gekomen om er aan te beginnen. Je eigenwijsheid, je hunkering naar kennis, je zelfstandigheid. Jij kon je ook ergens volledig in verdiepen en dan je eigen ideeën vormen en je eigen weg nemen.

Tijdens mijn promotietraject begon jij ook wetenschappelijke artikelen te schrijven, zonder enige opleiding daarvoor. Ik weet nog dat je me opbelde en volledig ontstemd was dat je aanpassingen moest maken aan je artikel voordat ze het wilde publiceren, en dat ik moest lachen en je uitlegde dat dat juist heel erg goed was. Toen werd je weer trots. Jouw eerste artikel werd zelfs eerder gepubliceerd dan het mijne. Ik ben verschrikkelijk trots op je dat je dat gelukt is, net als dat ik weet dat jij verschrikkelijk trots op mij bent dat ik ga promoveren.

Ons hoofdstuk is veel te vroeg afgesloten, maar ik zal doorgaan in jouw gedachte, out-of-thebox blijven denken en vertrouwen op mijn eigen kunnen.

Lieve papa,

Dankjewel.

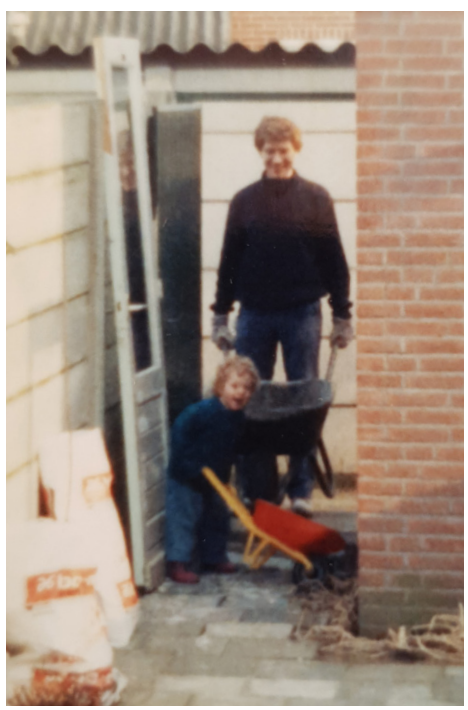




\section{Verder wil ik graag nog bedanken:}

ledereen die deel uit heeft gemaakt van mijn promotieteam:

Aalt, vanaf het moment dat ik voor mijn sollicitatiegesprek binnen kwam in jouw kantoor voelde ik me welkom. De wetenschappelijke discussies die wij samen konden voeren hebben mijn zelfvertrouwen erg gesterkt. Ik vond het ook geweldig om een half uur over de titel van een manuscript met je te kunnen discussiëren. Dankjewel dat je altijd mijn input en ideeën serieus nam en voor de vrijheid die ik altijd van je gekregen heb.

Antoon, naarmate het project vorderde kwam je meer in beeld. Ik vond het fijn dat je naast aandacht voor het inhoudelijke project, ook veel aandacht besteedde aan mijn ontwikkeling als onderzoeker en als mens. Bedankt voor alle opleidingsmogelijkheden en lessen rondom het project. Heel erg bedankt dat je altijd achter me stond, vooral in de moeilijkste periode. Ik kijk er dan ook naar uit om samen te blijven werken bij BuRO.

Misha, begonnen als AIO collega, geëindigd als co-promotor. Het is bijzonder gelopen! Als AIO-collega heel erg bedankt voor de gezellige lunches, koffies, borrels, onze tripjes naar BuRO, en de PET cursus in Wageningen. Ontzettend bedankt dat je me bent gaan helpen en uiteindelijk hebt toegezegd mijn co-promotor te zijn. Je bent een super co-promotor en ik weet zeker dat je nog veel toekomstige AIO's succesvol gaat begeleiden. Bedankt dat ik altijd over alles bij je terecht kon en kan.

Antje, dankjewel voor alles dat je me geleerd hebt, je Gründlichkeit, en onze gezellige gesprekken. Ik kon altijd bij je terecht en dat heb ik altijd ontzettend gewaardeerd.

Daarnaast wil ik graag de leden van mijn beoordelingscommissie bedanken: Prof. dr. Casper Schalkwijk, Prof. dr. Daisy Jonkers, Prof. dr. Dick Sijm, Prof. dr. Renger Witkamp, en dr. Hubert Deluyker.

Voordat ik aan dit AlO-traject begon zijn er een aantal docenten geweest in mijn Master opleiding die mij veel hebben geleerd, ondersteund, en me het vertrouwen hebben gegeven dat ik dit kon doen. Heel erg bedankt aan Renger Witkamp en Wilma Steegenga bij Wageningen University, and Lise Bjørkhaug Gundersen at the University of Bergen.

A massive thank you to all my past and present Phartox PhD colleagues for all the gezelligheid in the last 5 years: Alie, past roomie Charlotte, Christy, chatting and shopping buddy Ellen, George, crazy :) Gesiele, deep conversation buddy Julen, MTB buddy Kim, Kristien, LeTao, Merel, Ming, Mireille, my roomie Philippe, Quan, Rianne, Robert, Stefan, Shan, Sven, and Wenbo. 
En natuurlijk alle andere collega's van Phartox: Frederik-Jan: heel erg bedankt voor alle hulp, Agnes, Agnieszka, Agi, Alex M., Alex R. voor alle gezellige drankjes, Ben, Daniëlle, Geja, Ger, Gertjan, Guido, Harry, Helma, Jacques, Jan, Jos, Josephina, Lily, Lou voor alle praktische hulp, Marie-Claire, voor alle fijne gesprekken, Marie-José, Matthijs, Paul, Peter, Rian, Roger B: waar moet ik beginnen met jou te bedanken?, Roger G., en Sébastien.

De mannen van de spoelkeuken: Will en Alex, en Eddy voor het rondbrengen van de pakjes. ledereen bij WFSR die mij geholpen heeft: Stefan, Stanislava, Wouter, Astrid, en Toine ledereen bij Campus Venlo voor alle hulp en gezelligheid: Koen, Sanne, Jessica, Anouk, Cheng, Evy, Miriam, Tim, en natuurlijk Rob

My Maastricht family for all the love and laughter: Evelyn, Inge, Carey, Mirella, Frank, Julia, Daniela, Terezinha, Juan, Jana, Jasia, Anthony (and Caty) and including my fellow members of the PhD Academy social committee: Geert, Inēs, Jan, Martien, Mike, Nicolo, and Tate. Martijn voor alle koffietjes, en Guy voor de gezellige stapavonden en uitstap naar Carmen in Heidelberg.

Mijn schaatsmaatjes van de Limburgse Schaatsvrienden, met in het bijzonder Bram en Jorrit.

Ook heel erg bedankt aan de familie Cazemier en de familie van den Berg die mij altijd hebben ondersteund.

Mijn vrienden buiten Maastricht die me altijd zijn blijven opzoeken en waar ik veel fijne vakanties en tripjes mee heb beleefd: Anna en Sybren, Annelies en Tim, Dirk, Elske, Froukje en Tjalle, Frida en Sveinung, Imme en Teije, Joep en Eline, Jan en Miriam, Jeroen (ook voor het helpen met het idee van de cover), Jinthe, Linda, Loes, Mirthe, Rutger, en iedereen die ik misschien onverhoopt vergeten ben.

Of course my amazing and lovely paranymphs: Carmen and Bruna. Thank you for being my paranymphs, thank you for helping me to get to this defence through this horrible time. I love you both to bits. Ik kijk uit naar nog meer schnapps wandelingen in de Duitse heuvels Carmen! 
En natuurlijk mijn familie:

Lieve Roel en Olga, heel erg bedankt voor alle fijne gesprekken en goede tips van de afgelopen jaren. Het is erg fijn om te weten dat ik altijd bij jullie terecht kan en ik zal de komende jaren ook nog zeker vaak bij jullie aankloppen om lekker te eten en te kletsen.

Lieve Jard, dankjewel voor alles. En Luc ook uiteraard!

Lieve mama, natuurlijk draag ik dit boekje ook deels op aan jou. Dankjewel voor alles. Dankjewel dat je me altijd hebt gestimuleerd om echt een VWO-diploma te halen, en nu sta ik hier! :) 

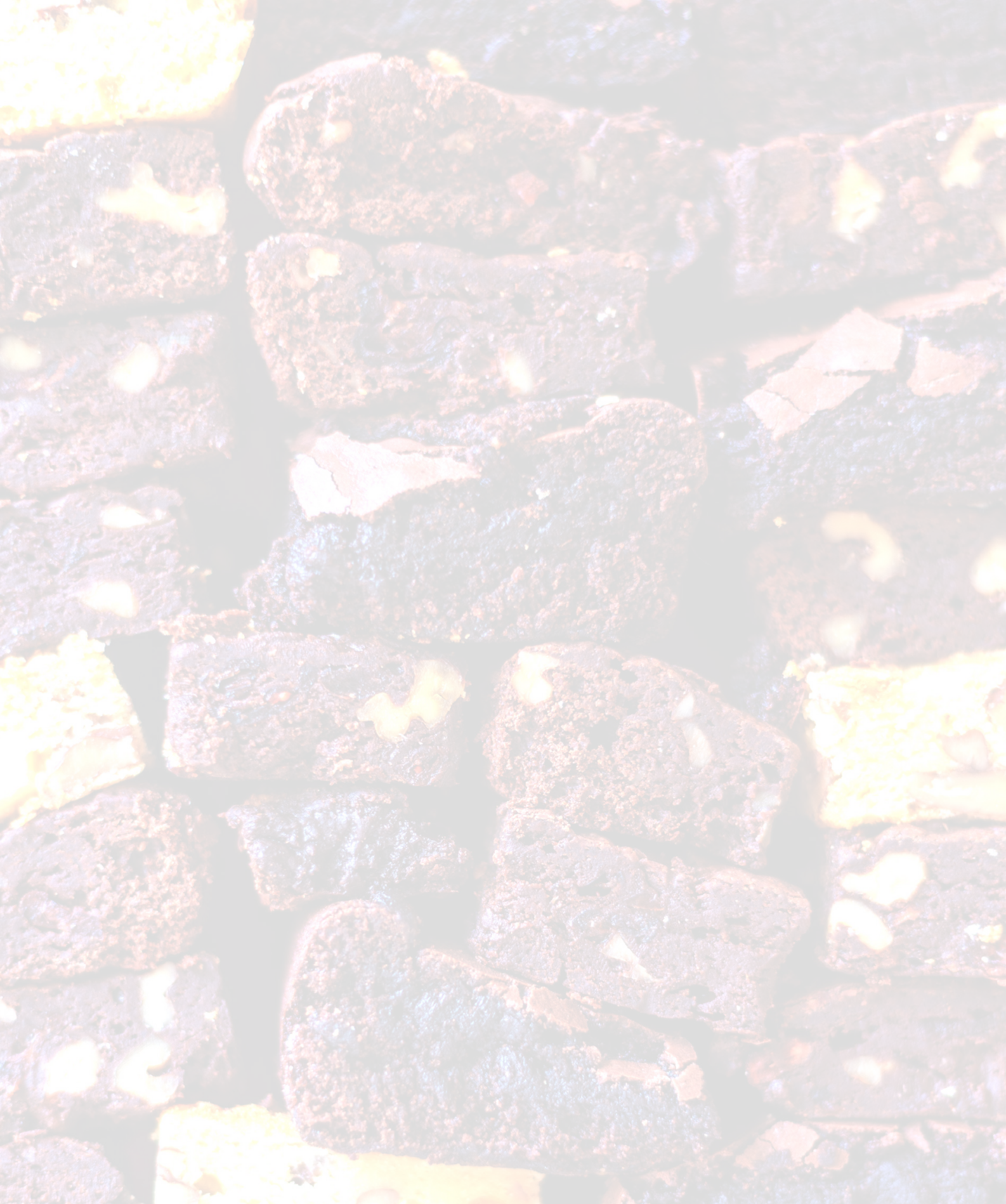


\title{
Addendum
}

\author{
Samenvatting \\ Valorisatie \\ Dankwoord \\ Curriculum Vitae \\ List of publications
}




\section{Curriculum Vitae}

Timme van der Lugt was born on February 17th, 1990 in Amsterdam, The Netherlands. After finishing pre-university education at secondary school 'Berger Scholengemeenschap' in Bergen (NH) in 2009, she enrolled in the bachelor program "Nutrition and Health" at Wageningen University, after graduating in 2012 she continued with the Master program "Nutrition and Health" with the specialization "Molecular Nutrition and Toxicology". During her master she worked at the Hormone Laboratory of the University of Bergen (Norway).

Upon obtaining her Master degree in 2014, she worked at the chairgroup Nutrition and Pharmacology of Wageningen University before starting her PhD research at the department of Pharmacology and Toxicology at Maastricht University. From May 2015 until May 2020 she investigated the pro-inflammatory potential of dietary advanced glycation endproducts, as described in this thesis, under the supervison of prof. dr. A. Bast, prof. dr. A. Opperhuizen, dr. M. F. Vrolijk and during her first years under the supervision of dr. A.R. Weseler. During this period, she presented her work at several national and international conferences and won a prize for best poster presentation at the annual NUTRIM symposium 2015. Additionally she has completed the post-graduate education in Toxicology. 

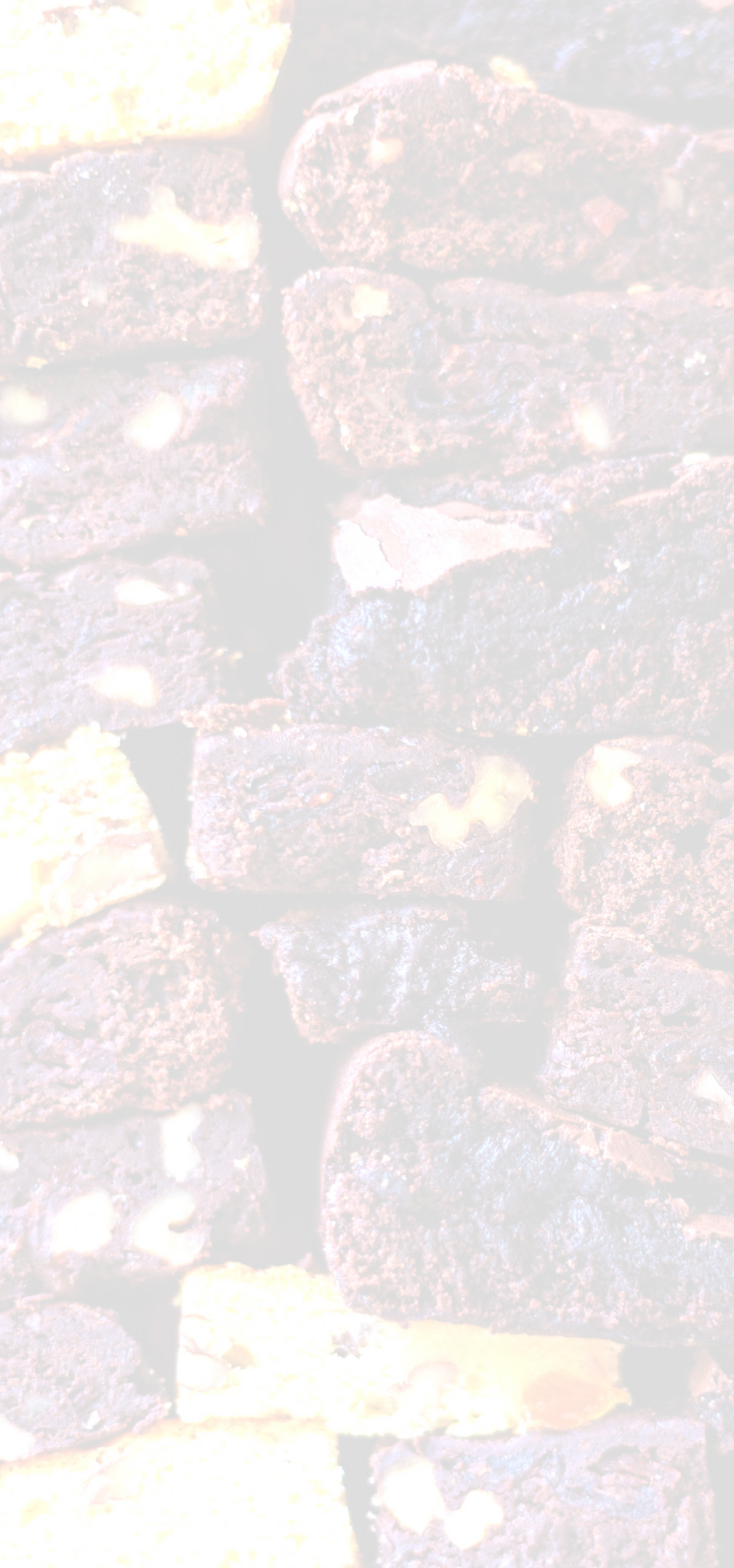

논 


\title{
Addendum
}

\author{
Samenvatting \\ Valorisatie \\ Dankwoord \\ Curriculum Vitae \\ List of publications
}




\section{Published}

1. T. van der Lugt, K. Venema, S. van Leeuwen, M. F. Vrolijk, A. Opperhuizen, A. Bast. Gastrointestinal digestion of dietary advanced glycation endproducts using an in vitro model of the gastrointestinal tract (TIM-1). Food \& Function, 2020, DOI: 10.1039/DOFO00450B

2. T. van der Lugt, A. R. Weseler, A. Opperhuizen, M. F. Vrolijk, A. Bast. Dietary advanced glycation endproducts decrease glucocorticoid sensitivity in vitro. Nutrients, 2020 Feb 10;12(2 ).pii: E441

3. T. van der Lugt, A. R. Weseler, W.A. Gebbink, M. F. Vrolijk, A. Opperhuizen, A. Bast. Dietary Advanced Glycation Endproducts Induce an Inflammatory Response in Human Macrophages in Vitro. Nutrients, 2018 Dec; 10(12): 1868

\section{Submitted/in preparation}

1. T. van der Lugt, A. Opperhuizen, A. Bast, M. F. Vrolijk. Dietary advanced glycation endproducts and the gastrointestinal tract: a review of the local effects. Submitted.

2. T. van der Lugt, A. R. Weseler, M. Suuring, M. F. Vrolijk, A. Opperhuizen, A. Bast. The biological effects of dietary advanced glycation endproducts are dependent on thermal treatment. In preparation.

3. T. van der Lugt, M. F. Vrolijk, T. F. H. Bovee, S. van Leeuwen, S. Vonsovic, A. Hamers, A. Opperhuizen, A. Bast. The pro-inflammatory potential of dietary advanced glycation endproducts after gastrointestinal digestion. In preparation.

4. S. Althari, L. A. Najmi, A. J. Bennett, I. Aukrust, J. K. Rundle, K. Colclough, J. Molnes, A. Kaci, S. Nawaz, T. van der Lugt, N. Hassanali, A. Molven, S. Ellard, M. I. McCarthy, L. Bjørkhaug, P. R. Njølstad, A. L. Gloyn. Unsupervised clustering of missense variants in the HNF1A gene using multidimensional functional data aids clinical interpretation. Under review. 


\section{Conference abstracts}

1. T. van der Lugt, M. F. Vrolijk, T. F. H. Bovee, S. van Leeuwen, A. Opperhuizen, A. Bast. Dietary advanced glycation endproducts keep their pro-inflammatory potential after gastrointestinal digestion. Young AGErs symposium 2020, Dresden, Germany (oral presentation, pending due to Corona pandemic).

2. T. van der Lugt, K. Venema, S. van Leeuwen, M. F. Vrolijk, A. Opperhuizen, A. Bast. Using the TNO gastrolntestinal Model to assess the effect of small intestinal digestion on the pro-inflammatory characteristics of dietary advanced glycation endproducts. Society of Toxicolog meeting 2020, Anaheim, USA (poster presentation, pending due to Corona pandemic).

3. T. van der Lugt, K. Venema, S. van Leeuwen, M. F. Vrolijk, A. Opperhuizen, A. Bast. Gastrointestinal digestion of dietary advanced glycation endproducts in vitro. Annual NUTRIM symposium 2019, Maastricht, The Netherlands (poster presentation).

4. T. van der Lugt, K. Venema, S. van Leeuwen, M. F. Vrolijk, A. Opperhuizen, A. Bast. The effect of small intestinal digestion on dietary advanced glycation endproducts. Young AGErs symposium 2019, Maastricht, The Netherlands (oral presentation).

5. T. van der Lugt, A. R. Weseler, M. F. Vrolijk, A. Opperhuizen, A. Bast. Dietary advanced glycation endproducts and glucocorticoid resistance, are the two linked? Eurotox Congress 2019, Helsinki, Finland (poster presentation).

6. T. van der Lugt, A. R. Weseler, A. Opperhuizen, A. Bast. Food-derived Advanced Glycation End Products Induce An Inflammatory Response In Human Macrophages: Implications For Human Health? Society of Toxicology meeting 2018, San Antonio, USA (poster presentation).

7. T. van der Lugt, A. R. Weseler, A. Opperhuizen, A. Bast. Dietary advanced glycation endproducts and glucocorticoid resistance, are the two linked? Nederlandse Verening voor Toxicologie Meeting 2018, Hilversum, The Netherlands (poster presentation).

8. T. van der Lugt, A. R. Weseler, A. Opperhuizen, A. Bast. Dietary advanced glycation endproducts cause glucocorticoid resistance. Annual NUTRIM symposium 2018, Maastricht, The Netherlands (poster presentation).

9. T. van der Lugt, A. R. Weseler, A. Opperhuizen, A. Bast. EFood-derived Advanced Glycation End Products Induce An Inflammatory Response In Human Macrophage-like Cells: Implications For Human Health? Society of Toxicology meeting 2017, Baltimore, USA (poster 
presentation).

10. T. van der Lugt, A. R. Weseler, A. Opperhuizen, A. Bast. Effect of food-derived advanced glycation end products on macrophage-like cells: implications for human health? Eurotox Congress 2016, Sevilla, Spain (poster presentation).

11. T. van der Lugt, A. R. Weseler, A. Opperhuizen, A. Bast. Food-derived advanced glycation end products induce an inflammatory response in human macrophages in vitro. 3rd World Congress on Maillard Reaction \& Glycation 2016, Budapest, Hungary (poster presentation)

12. T. van der Lugt, A. R. Weseler, A. Opperhuizen, A. Bast. Do food-derived advanced glycation end products affect human health? Annual NUTRIM symposium 2016, Maastricht, The Netherlands (oral presentation).

13. T. van der Lugt, A. R. Weseler, A. Opperhuizen, A. Bast. Is there an effect of foodderived advanced glycation end products on human health? Annual NUTRIM symposium 2015, Maastricht, The Netherlands (poster presentation). 